\title{
LOW POWER 10-BIT ANALOG TO DIGITAL CONVERTER
}

\author{
By \\ LADO FILIPOVIC
}

\author{
A thesis \\ presented to \\ Carleton University and OCIECE \\ in fulfilment of the \\ thesis requirement for the degree of \\ MASTER OF APPLIED SCIENCE \\ in \\ ELECTRICAL ENGINEERING
}

Ottawa, Ontario, Canada

(C) 2009 Lado Filipovic 
Library and

Archives Canada

Published Heritage

Branch

395 Wellington Street

Ottawa ON K1A 0N4

Canada
Bibliothèque et

Archives Canada

Direction du

Patrimoine de l'édition

395 , rue Wellington

Ottawa ON K1A ON4

Canada
NOTICE:

The author has granted a nonexclusive license allowing Library and Archives Canada to reproduce, publish, archive, preserve, conserve, communicate to the public by telecommunication or on the Internet, loan, distribute and sell theses worldwide, for commercial or noncommercial purposes, in microform, paper, electronic and/or any other formats.

The author retains copyright ownership and moral rights in this thesis. Neither the thesis nor substantial extracts from it may be printed or otherwise reproduced without the author's permission.
Your file Votre référence ISBN: 978-0-494-52035-2 Our file Notre référence ISBN: 978-0-494-52035-2
In compliance with the Canadian

Privacy Act some supporting forms may have been removed from this thesis.

While these forms may be included in the document page count, their removal does not represent any loss of content from the thesis.
AVIS:

L'auteur a accordé une licence non exclusive permettant à la Bibliothèque et Archives Canada de reproduire, publier, archiver, sauvegarder, conserver, transmettre au public par télécommunication ou par l'Internet, prêter, distribuer et vendre des thèses partout dans le monde, à des fins commerciales ou autres, sur support microforme, papier, électronique et/ou autres formats.

L'auteur conserve la propriété du droit d'auteur et des droits moraux qui protège cette thèse. $\mathrm{Ni}$ la thèse ni des extraits substantiels de celle-ci ne doivent être imprimés ou autrement reproduits sans son autorisation.

\section{Canadä}




\section{Abstract}

A 10-bit successive approximation analog-to-digital converter (ADC), with offset correction circuitry and a tunable series attenuation capacitor is presented. The ADC is designed in a mixed signal $0.13 \mu \mathrm{m}$ CMOS process technology and operates with supply voltages down to $0.4 \mathrm{~V}$. The ADC uses MOSFETs that are designed to operate in the sub-threshold region of operation. The ADC achieved sample rates of up to $500 \mathrm{kS} / \mathrm{s}$ when a voltage supply of $1.2 \mathrm{~V}$ was used, resulting in an ADC dynamic performance of $60.7 \mathrm{~dB}$ SINAD and $9.8 \mathrm{ENOB}$, while dissipating $19.71 \mu \mathrm{W}$ of power and $39.4 \mathrm{pJ} /$ cycle of energy. An energy per cycle of $28.8 \mathrm{pJ} /$ cycle was measured while operating at $10 \mathrm{kS} / \mathrm{s}$, with a $0.4 \mathrm{~V}$ supply voltage. A series attenuation capacitor was used to reduce the size of the circuit. Since processing variations can change the value of this capacitor and degrade the ADC operation, the series attenuation capacitor was designed to vary between $572 \mathrm{fF}$ to $694 \mathrm{fF}$, controllable using five digital input bits. The optimal variable capacitor code was designed to be $647 \mathrm{fF}$ and a digital algorithm was designed to control the value of the attenuation capacitor to accommodate for process variations. This was done by analyzing the signal to noise and distortion ratio (SINAD) at the output of the ADC, and selecting the optimal capacitor code to maximize SINAD. 


\section{Acknowledgements}

I would like to thank my family, including my Dad and Mom, as without them, nothing in my life, least of which is this thesis, would be possible. Also, a big thank you to my big sister, Lidija, for her love, advice, and support.

Foremost, my supervisor Dr. Leonard MacEachern for his advice and assistance while working on this masters degree.

I would also like to thank Nagui Mikhail for his invaluable help in setting up my test equipment as well as his assistance throughout my undergraduate and masters degrees. Another thank you goes to Blazenka Power whose advice was very helpful through some difficult times.

Next, I wish to thank Karim Abdel-Halim and Igor Miletic for their constant support and advice, both technical and personal while working on this thesis. In addition, I would like to thank Till Kuendiger, Ghyslain Gagnon, Ziad El-Khatib and Sinisa Milicevic for their immense help during the highly stressful tapeout phase of the masters. I would also like to extend my thanks to Greg Brzezina and Oleksandr Tkachenko for their help and friendship during the years of my masters degree.

Also NSERC, OGS, and CMC for funding the research presented in the thesis. 


\section{Table of Contents}

$\begin{array}{lll}\text { Abstract } & \text { iii }\end{array}$

Acknowledgements iv

Table of Contents $\quad$ v

List of Tables $\quad$ viii

List of Figures $\quad$ x

List of Abbreviations xvi

1 Introduction 1

1.1 Motivation . . . . . . . . . . . . . . . . . 1

1.2 Thesis Organization . . . . . . . . . . . . . . . . 3

2 ADC Specifications $4 . \ldots \ldots$

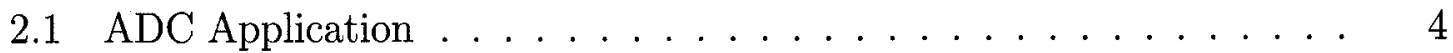

2.1.1 Biomedical Transmitter . . . . . . . . . . . . . 5

2.1.2 Wireless Sensor Network . . . . . . . . . . . . . 7

2.2 Design Specifications $\ldots \ldots \ldots \ldots$

2.3 Summary of ADC Specifications . . . . . . . . . . . 10

3 Background $\quad 12$

3.1 ADC Architectures . . . . . . . . . . . . . . . . . . . 12

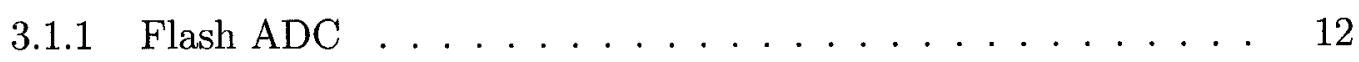

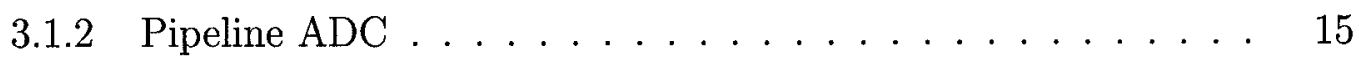

3.1 .3 Integrating ADC . . . . . . . . . . . . . . . . . 17

Single-Slope Architecture . . . . . . . . . . . . . . . 17

Dual-Slope Architecture . . . . . . . . . . . . . . . 18

$3.1 .4 \quad$ Sigma-Delta $(\Sigma \Delta)$ ADC $\ldots \ldots \ldots \ldots$

3.1.5 Successive Approximation ADC . . . . . . . . . . . 20

3.2 Low-Power Design Architectures . . . . . . . . . . . . . . . . 23

3.3 Background Summary . . . . . . . . . . . . . . . 26 
4 ADC Circuit Architecture 27

4.1 Successive Approximation ADC Performance . . . . . . . . . 27

4.2 ADC Components . . . . . . . . . . . . . . . . . . 29

4.2.1 Digital Successive Approximation Register (SAR) . . . . . 30

4.2 .2 Comparator . . . . . . . . . . . . . . . . . . 31

4.2 .3 Capacitor Array . . . . . . . . . . . . . . . . . . . 31

4.2.4 Switching Network for the Capacitor Array . . . . . . . . . . 32

4.2.5 Switches for the Top Plate of the Capacitor Array . . . . . . 32

4.2 .6 Output Buffers . . . . . . . . . . . . . . 32

4.3 ADC Circuit Architecture Summary . . . . . . . . . . . 33

5 SAR ADC Design Components 35

5.1 Comparator Architecture . . . . . . . . . . . . . . 35

5.1 .1 Comparator Design . . . . . . . . . . . . . . . . . 35

5.1 .2 Offset Voltage for the Comparator . . . . . . . . . . . . 37

5.1 .3 Offset Voltage Correction Circuit . . . . . . . . . . . . . 38

5.1.4 Offset Voltage for the Two-Stage Comparator . . . . . . . . 42

5.1 .5 Comparator Simulations . . . . . . . . . . . . . . 45

Bias Current, Power, and Bandwidth Simulation . . . . . . . . 45

Temperature Simulation . . . . . . . . . . . . . 46

5.1 .6 Comparator Layout . . . . . . . . . . . . . . . . . 47

5.2 Successive Approximation Register Design . . . . . . . . . . . 47

5.2 .1 SAR Operation . . . . . . . . . . . . . . . . 48

5.2 .2 SAR Power Dissipation . . . . . . . . . . . . . . . 53

5.3 Binary Weighted Capacitor Array Design . . . . . . . . . . . 55

5.3.1 Tunable Attenuation Capacitor Design . . . . . . . . . . 62

5.3.2 Binary Weighted Capacitor Array Layout . . . . . . . . . . . . 64

5.4 Analog Switches and Output Buffers . . . . . . . . . . . 67

5.4.1 Design of the Analog Switches for the SAR ADC $\ldots \ldots . .67$

5.4.2 Design of the Output Buffers for the SAR ADC . . . . . . 70

5.5 10-bit Analog to Digital Converter with a Tunable Capacitor Array . 70

5.6 ADC Design Summary . . . . . . . . . . . . . . . 71

6 Simulation Results of the ADC $\quad \mathbf{7 5}$

6.1 Linearity Simulated Results . . . . . . . . . . . . . . . . 75

6.1.1 ADC Attenuation Capacitor Tuning . . . . . . . . . . 80

6.2 Dynamic Simulated Results . . . . . . . . . . . . . . . . 80

6.3 Power Dissipation . . . . . . . . . . . . . . . . . . . . 84

6.4 Summary of Simulated Results $\ldots \ldots \ldots \ldots$

7 Measured Results $\quad 90$

7.1 Measurement Description . . . . . . . . . . . . . . . . . . . 90 90

7.2 Measurement Setup . . . . . . . . . . . . . . . . . 94 
7.3 Dynamic Measured Results . . . . . . . . . . . . . . . . . . . . 95

7.3.1 Varying the Attenuation Capacitor . . . . . . . . . . . . 104

7.4 Power Dissipation . . . . . . . . . . . . . . . . . . . . 110

7.5 Summary of the Measured ADC Results . . . . . . . . . . . 115

7.6 Comparison to Published ADCs . . . . . . . . . . . . 117

8 Conclusion $\quad 120$

8.1 Contributions and Achievements . . . . . . . . . . . . . 120

8.2 Drawbacks of Presented Design . . . . . . . . . . . . . 122

8.3 Future Work . . . . . . . . . . . . . . . . . . . . . 123

$\begin{array}{ll}\text { A Sub-Threshold Design } & 127\end{array}$

A.1 Sub-Threshold MOSFET Operation . . . . . . . . . . . . . 127

A.1.1 Analog Design in Subthreshold . . . . . . . . . . . . . 131

A.1.2 Digital Design in Subthreshold . . . . . . . . . . . . . 133

$\begin{array}{ll}\text { B Resulting Publications } & 137\end{array}$

$\begin{array}{ll}\text { Bibliography } & 138\end{array}$ 


\section{List of Tables}

2.1 Summary of the desired specifications for the proposed ADC. . . . . 11

3.1 Published Low-Power ADC Designs. . . . . . . . . . . . . . 25

5.1 Sizes for the devices used for the presented comparator . . . . . . . 41

5.2 Simulated bias current, power, and bandwidth of the comparator. . . 46

5.3 Variation in comparator offset voltage due to temperature variation. . $\quad 47$

5.4 Explanation for the input and output signals of the successive approximation register from Figure 5.8. . . . . . . . . . . . 51

5.5 Settling time for charging each bit capacitor at $V_{D D}=0.6 \mathrm{~V} . \ldots . \quad 69$

5.6 Summary of the ADC pins. . . . . . . . . . . 73

6.1 Summary of ADC performance at $0.6 \mathrm{~V}$ and $1.2 \mathrm{~V} V_{D D} \ldots \ldots$. . . . 84

6.2 Summary of power consumption vs. $V_{D D} \ldots \ldots \ldots$. . . . 87

7.1 ADC Setup when testing attenuation capacitor code variation. . . . . 105

7.2 ADC Setup when testing attenuation capacitor code variation. . . . 108

7.3 Summary of measured power consumption vs. $V_{D D} \ldots \ldots \ldots$. . . . 114

7.4 Summary of power dissipation vs. $V_{d d}$ when the sample rate is $25 \mathrm{kS} / \mathrm{s} .115$

7.5 Summary of the measured ADC Results. . . . . . . . . . . . . . . 119

viii 
7.6 Comparison of the presented design with published 10-bit ADC designs.119 


\section{List of Figures}

2.1 Voltage and frequency ranges for various biomedical signals. . . . . . 6

2.2 Sample block diagram of a biomedical transmitter. . . . . . . . . 8

2.3 Serial topology for a wireless sensor network [1] . . . . . . . . 9

3.1 Block diagram of a Flash ADC. . . . . . . . . . . . 13

3.2 Block diagram of a Pipeline ADC . . . . . . . . . . . . . 16

3.3 Block diagram of a single-slope Integrating ADC. . . . . . . . . . 18

3.4 Block diagram of a dual-slope Integrating ADC. . . . . . . . . . . . . 19

3.5 Typical block diagrams for the (a) Nyquist rate ADC and (b) Oversampling ADC. ........................ 20

3.6 Block diagrams of two sigma-delta modulators. . . . . . . . . . . . 21

3.7 Successive approximation register ADC block diagram and flow chart. 22

4.1 Sampling phase of the SAR ADC. . . . . . . . . . . . 28

4.2 Sampling phase of the SAR ADC during which the top plate of the capacitor becomes $V_{D D}-V_{i n} \ldots \ldots \ldots \ldots$

4.3 Approximation phase of the SAR ADC, with the MSB approximation step shown . . . . . . . . . . . . . . . 
4.4 Approximation phase of the SAR ADC, with the MSB approximation

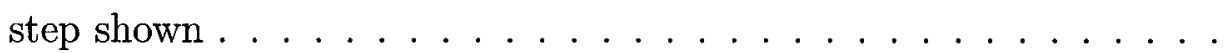

5.1 Schematic for the first stage of the comparator design. . . . . . . . . 36

5.2 Offset voltage for the presented comparator. . . . . . . . . . . . 38

5.3 Circuit of the complete comparator, showing two stages, including an offset voltage correction circuit. . . . . . . . . . . . . . 39

5.4 Timing of the Precharge and RST signals for the offset voltage correction circuit. . . . . . . . . . . . . . . . 42

5.5 Offset voltage of the presented comparator after implementing the offset correction circuit. . . . . . . . . . . . . . . . .

5.6 Offset voltage of the comparator shown before and after the offset voltage correction circuit is implemented as a result of a varying Tune voltage. ......................... 44

5.7 Layout of the comparator circuit. . . . . . . . . . . . . . . . 48

5.8 SAR circuit including shift registers. . . . . . . . . . . . . . 49

5.9 Reset and control circuit from Figure 5.8. . . . . . . . . . . . . . 50

5.10 D-flip-flop design used in the successive approximation register from Figure $5.8 \ldots \ldots \ldots \ldots \ldots$. . . . . . . . . . . . . . 50

5.11 Timing of the SAR input and output signals during a conversion cycle. 52

5.12 Complete layout of the SAR circuit along with the digital circuit for the control circuitry from Figure $5.8 . \ldots \ldots \ldots 4$ 
5.13 Total digital power dissipation of the SAR with varying supply voltages at a frequency of $1.4 \mathrm{MHz}$, corresponding to a sample rate of $100 \mathrm{kS} / \mathrm{s}$.

5.14 Total digital power dissipation of the SAR with varying input frequency at a $0.6 \mathrm{~V}$ power supply. . . . . . . . . . . . 57

5.15 Monte Carlo statistical analysis simulation of the SAR digital power. Simulation performed for 100 runs with process variation and mismatch, a $V_{D D}$ at $0.6 \mathrm{~V}$, and a clock at $1.4 \mathrm{MHz} \ldots \ldots . . . . . .58$

5.16 Binary weighted capacitor array schematic for a 10-bit ADC. . . . . 58

5.17 Thermal noise of a capacitor at room temperature $(\mathrm{T}=300 \mathrm{~K}) . \quad \ldots \quad 60$

5.18 Binary weighted capacitor array circuit including analog switches. . . 61

5.19 Schematic of the digitally tunable attenuation capacitor, $C_{A T T} \ldots \ldots$. 64

5.20 Layout of the tunable attenuation capacitor at an area of $255 \mu \mathrm{m}$ by $140 \mu m \ldots \ldots \ldots \ldots \ldots \ldots \ldots \ldots \ldots \ldots \ldots \ldots \ldots \ldots \ldots \ldots \ldots \ldots$

5.21 Layout of the capacitor array with dimensions of $290 \mu \mathrm{m}$ by $230 \mu \mathrm{m}$, together with the tunable attenuation capacitor (Total area of $290 \mu \mathrm{m}$

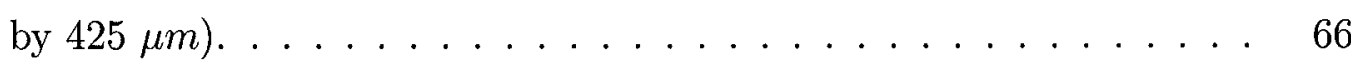

5.22 Schematic showing the switches in the ADC design. . . . . . . . 68

5.23 The three switches' ON resistance at various supply voltages. . . . . . 69

5.24 Schematic showing the output buffer driven by the Control signal. . . 70

5.25 Complete layout of the designed ADC with dimensions of $1.25 \mathrm{~mm} \mathrm{x}$

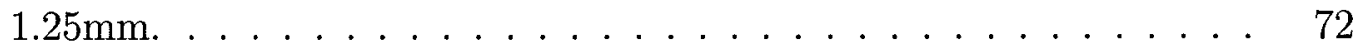

6.1 Sample DNL and INL measurements. . . . . . . . . . . . . . . . . 76 
6.2 Digital output superimposed on the analog input for the ADC at $100 \mathrm{kS} / \mathrm{s}$ with a $V_{D D}=0.6 \mathrm{~V} \ldots \ldots \ldots \ldots 77$

6.3 INL and DNL plots for all codes at $100 \mathrm{kS} / \mathrm{s}, \mathrm{VDD}=0.6 \mathrm{~V} \ldots \ldots . . .78$

6.4 INL and DNL plots for all codes at $320 \mathrm{kS} / \mathrm{s}, \mathrm{VDD}=1.0 \mathrm{~V} \ldots \ldots . . . \quad 79$

6.5 INL and DNL plots for all codes at $500 \mathrm{kS} / \mathrm{s}, \mathrm{VDD}=1.2 \mathrm{~V} \ldots \ldots$. . . . 80

6.6 INL and DNL plots for all codes at $500 \mathrm{kS} / \mathrm{s}, \mathrm{VDD}=1.2 \mathrm{~V}, \mathrm{Vref}=0.6 \mathrm{~V} \quad 81$

6.7 Plot of the output from the ADC when a $200 \mathrm{~Hz}$ sinusoid is applied to the input, sampled at $500 \mathrm{kS} / \mathrm{s}$, with a $V_{D D}=1.2 \mathrm{~V} \ldots \ldots . . \ldots 2$

6.8 Plot of the output from the ADC when a $20 \mathrm{~Hz}$ sinusoid is applied to the input, sampled at $100 \mathrm{kS} / \mathrm{s}$, with a $V_{D D}=0.6 \mathrm{~V} \ldots \ldots . \ldots 85$

6.9 Power dissipation of the $\mathrm{ADC}$ with varying $V_{D D} \ldots \ldots \ldots$

6.10 Energy per cycle of the ADC with varying $V_{D D} \ldots \ldots$. . . . . 87

7.1 Complete fabricated ADC with pins labeled. . . . . . . . . . . . 91

7.2 (a) Fabricated ADC bonded to QFN32 package and (b) Basic test setup for the ADC $(\mathrm{DUT}) . \ldots \ldots \ldots$. . . . . . . . . . . . . . 91

7.3 Sample SFDR measurement from an FFT plot [2] . . . . . . . . . . 92

7.4 Sample SNR measurement from an FFT plot [2]. . . . . . . . . . . 93

7.5 Effects of non-linearities in an A/D converter [2]. . . . . . . . 93

7.6 Test setup for the ADC. . . . . . . . . . . . . 95

7.7 Layout of the designed PCB. . . . . . . . . . . . . 96

7.8 Labeled photograph of the PCB. . . . . . . . . . . . . 97

7.9 Measured SFDR for the ADC. . . . . . . . . . . . . 101 
7.10 Measured SNR and SINAD for the ADC. . . . . . . . . . . . . . 102

7.11 Measured ENOB for ADC. . . . . . . . . . . . . . 103

7.12 Effect of varying the attenuation capacitor code on the capacitance. . 104

7.13 Varying the Capacitor Code - Includes Full Nyquist Bandwidth. . . . 107

7.14 Varying the Capacitor Code. . . . . . . . . . . . . . . . 111

7.15 Measured power for ADC. . . . . . . . . . . . . . . 112

7.16 Measured energy for ADC. . . . . . . . . . . . . . . 113

7.17 Effect of varying the voltage supply on the digital power, with sample rate $=25 \mathrm{kS} / \mathrm{s} \ldots \ldots \ldots \ldots \ldots \ldots \ldots \ldots$

8.1 Simple 3-bit capacitor tuning topology. . . . . . . . . . . . . . 124

8.2 Block diagram of a circuit that implements on-chip capacitor tuning. $\quad 125$

A.1 $I_{D S}$ vs. $V_{G S}$ simulation for an NFET (W=1 $\left.\mu m, \mathrm{~L}=250 \mathrm{~nm}, V_{D S}=1.2 \mathrm{~V}\right) 128$

A.2 Cross section of an n-type MOSFET in the subthreshold regime. . . . 129

A.3 $I_{D S}$ vs. $V_{D S}$ simulation for an NFET. $\left(\mathrm{W}=1 \mu m, \mathrm{~L}=250 \mathrm{~nm}, V_{G S}=0.2 \mathrm{~V}\right) 133$

A.4 Voltage Transfer Characteristics (VTC) for an inverter with varying $V_{D S}$ voltages. The inverter is a CMOS inverter with an NMOS transistor with $\mathrm{W}=1 \mu \mathrm{m}, \mathrm{L}=250 \mathrm{~nm}$, and PMOS transistor with $\mathrm{W}=3.5 \mu \mathrm{m}$, $\mathrm{L}=250 n m \ldots \ldots \ldots \ldots \ldots \ldots \ldots \ldots$

A.5 Drain current for an inverter while sweeping $V_{g s}$ with various $V_{D S}$ voltages. The inverter is a CMOS inverter with an NMOS transistor with $\mathrm{W}=1 \mu m, \mathrm{~L}=250 \mathrm{~nm}$, and PMOS transistor with $\mathrm{W}=3.5 \mu m, \mathrm{~L}=250 \mathrm{~nm} .135$ 
A.6 Capacitor components for an n-type MOSFET in the subthreshold regime [3]. $C_{O X}$ is the oxide capacitance, $C_{O f}$ and $C_{i f}$ are fringing capacitances, $C_{d o}$ are the drain and source overlap capacitances, $C_{d}$ is the depletion region capacitance, and $C_{J}$ is the junction capacitance. $\quad 136$ 


\section{List of Abbreviations}

$\begin{array}{ll}\text { ADC } & \text { Analog-to-Digital Converter } \\ \text { AHDL } & \text { Analog Hardware Description Language } \\ \text { BJT } & \text { Bipolar Junction Transistor } \\ \text { CMOS } & \text { Complementary Metal Oxide Semiconductor } \\ \text { DAC } & \text { Digital-to-Analog Converter } \\ \text { DC } & \text { Direct Current } \\ \text { DFT } & \text { Discrete Fourier Transform } \\ \text { DIP } & \text { Dual In-line Package } \\ \text { DNL } & \text { Differential Nonlinearity } \\ \text { DUT } & \text { Device Under Test } \\ \text { ECG } & \text { Electrocardiogram } \\ \text { ENOB } & \text { Effective Number of Bits } \\ \text { ESD } & \text { Electro-Static Discharge } \\ \text { FFT } & \text { Fast Fourier Transform } \\ \text { IC } & \text { Integrated Circuit } \\ \text { INL } & \text { Integral Nonlinearity } \\ \text { LSB } & \text { Least-Significant Bit }\end{array}$




\begin{tabular}{|c|c|}
\hline MIMcap & Metal-Insulator-Metal Capacitor \\
\hline MOSFET & Metal Oxide Semiconductor Field Effect Transistor \\
\hline MSB & Most-Significant Bit \\
\hline NFET & N-type Field Effect Transistor \\
\hline PCB & Printed Circuit Board \\
\hline PGP & Power Generation Photodiode \\
\hline PFET & P-type Field Effect Transistor \\
\hline PSD & Power Spectral Density \\
\hline QFG & Quasi-Floating-Gate \\
\hline $\mathrm{RSD}$ & Redundant Signed Digit \\
\hline SAR & Successive Approximation Register \\
\hline SINAD & Signal to Noise and Distortion Ratio, (also called SNDR) \\
\hline SFDR & Spurious Free Dynamic Range \\
\hline SMA & Sub-Miniature Type A (cable connector) \\
\hline SNR & Signal-to-Noise Ratio \\
\hline THD & Total Harmonic Distortion \\
\hline VDD & Positive supply voltage \\
\hline VDS & Drain-Source voltage of a MOSFET \\
\hline VGS & Gate-Source voltage of a MOSFET \\
\hline VREF & Reference voltage used by an ADC \\
\hline VSS & Negative supply voltage \\
\hline VTC & Voltage Transfer Characteristic \\
\hline
\end{tabular}

xvii 


\section{Chapter 1}

\section{Introduction}

\subsection{Motivation}

Medical advancements have reached a level where a patient's well-being and vital signs can be constantly monitored. The life of a patient can depend on early detection of any signs of medical trouble. Instead of waiting for symptoms to arrive to begin life-saving procedures, patients can be monitored continuously with implantable sensors [4]. Implantable medical devices are used in the diagnosis, cure, mitigation, treatment, or prevention of a disease. Examples of such devices are pacemakers, artificial organs, artificial limbs, and cochlear implants. These devices may need to be able to read and manipulate data, while being implanted in the human body with a battery power supply. Since implantable wireless sensors may be battery-powered, there is a desire to create low-power circuitry in order to extend the battery life of implanted sensors to prevent frequent invasive battery-replacement procedures for the patients.

An Analog to Digital Converter (ADC) is an essential component for communication between any implanted sensor with an analog output signal and digital data processing and analysis software. In the biomedical engineering field, there is a high 
demand for low-power ADCs with 8-12 bit resolution. These devices generally need low to medium sample rates, at approximately $1 \mathrm{kS} / \mathrm{s}$ to $1 \mathrm{MS} / \mathrm{s}[5]$.

Low-power devices are also essential in wireless sensor networks. Sensors are commonly placed at remote locations for extended periods of time, and are powered using ambient sources, such as solar power, or with a battery pack. Having a lowpower ADC to convert the sensed analog data to digital data is essential to the capability of the sensor to perform data analysis at the remote location [6]. The sensors commonly used in wireless sensor networks require medium sample rates in the hundreds of $\mathrm{kS} / \mathrm{s}[7]$.

Along with biomedical advantages and wireless sensor network applications of low power design, low-power devices are desirable in commercial electronics. Portable devices, such as MP3 players, PDA devices, laptops and cell phones need to be power-conscious if they wish to be competitive in the market. There is a constant drive to increase the functionality of these devices, while simultaneously reducing power consumption. Mixed-signal devices require ADCs, and improving their power consumption increases the product's commercial value.

Therefore, the goal of this thesis is to design a fully integrated 10-bit ADC in a standard CMOS process that is competitive with current architectures in its power consumption and energy-per-cycle ratio. The ADC is designed to operate in a bandwidth suitable for biomedical applications, up to approximately $10 \mathrm{kHz}[5]$. 


\subsection{Thesis Organization}

Chapter 2 gives an overview of the intended application of the designed ADC and will give specifications that will be targeted with the presented design.

Chapter 3 presents various options for ADC architectures in order to understand which architecture best suits the desired specifications. The selection of the successive approximation ADC with charge redistribution will be justified, and various published designs will be discussed and summarized.

Chapter 4 presents the chosen successive approximation ADC architecture and the main design components will be discussed briefly.

Chapter 5 analyzes the the design process in detail, as the individual components of the ADC are analyzed and explained.

Chapter 6 presents the post-layout simulation results, including parasitic capacitances and resistances. This includes the static performance, dynamic performance, and power dissipation simulations.

Chapter 7 describes the test procedure and shows the test setup used for the measurements. It also presents the measured results of the ADC, including the dynamic performance, and power dissipation.

Chapter 8 concludes the thesis, summarizes the work's contribution to research, and delves into possible suggestions for future evolution of the work. 


\section{Chapter 2}

\section{ADC Specifications}

\subsection{ADC Application}

Analog-to-digital converters (ADC) are used in a wide range of applications. Its function is to convert a continuous analog signal to a discrete digital representation, enabling further processing on the obtained data. An example of an analog signal that requires conversion to digital is an output from a physical sensor. Sensors, such as those used in the biomedical field, have an analog output signal that represents a physical event. In order to be able to process the information obtained from the sensor to do further analysis on it, the analog signal needs to be converter to a digital representation. This work proves applicable in fields requiring analog to digital conversion with low power dissipation, such as biomedical sensor design and wireless sensor networks.

One of the intended applications of the proposed successive approximation ADC design is for implantable biomedical sensors. A biomedical sensor converts a physical measurement in the human body such as a heart signal, blood sugar levels, or blood 
pressure, into an analog signal. This signal is then converted to digital bits and analyzed with a DSP circuit block. More information on the workings of a biomedical transmitter is shown in Section 2.1.1. The frequency range that is required for various biomedical signals can vary from DC to approximately $10 \mathrm{kHz}$, as is shown in Figure 2.1 [8]. Biomedical signals shown in Figure 2.1 are electrooculogram (EOG), electroencephalogram (EEG), electrocardiogram (ECG), electromyogram (EMG), and axon action potential (AAP) [8].

The proposed low power ADC can also be applied in the design of wireless sensor networks. Wireless sensor networks require low-power sensors that are deployed at remote locations and use ambient sources of power, or are equipped with a battery pack. The data processing capability at the remote sensor location is an essential component of decentralized sensor networks [6]. In order to perform digital data processing, an $\mathrm{ADC}$ is required to convert the sensed data to a digital form, while dissipating minimal power.

\subsubsection{Biomedical Transmitter}

The targeted application requires circuitry that dissipates the least possible amount of power. In implantable sensors, any potential battery pack that is implanted in the human body would require a surgical procedures to replace. With low-power ADC designs, there is also a hope to power the devices using sources other than a battery pack. Recent advancements in technology have suggested that there is potential in the sensor becoming self-powered. The use of ambient energy to power low-power designs was analyzed in [9], where it was shown that power in the order of $400 \mu W$ 


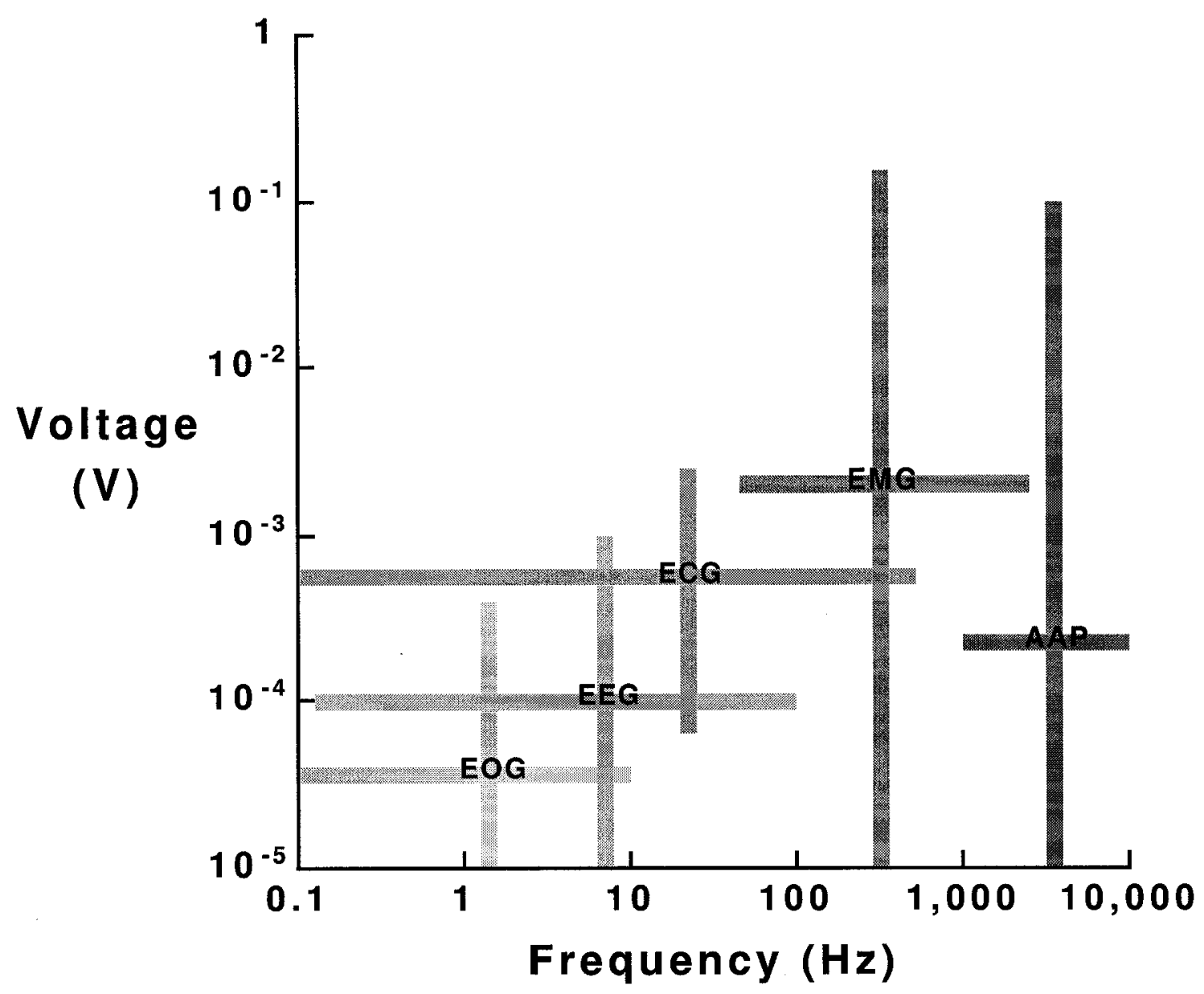

Figure 2.1: Voltage and frequency ranges for various biomedical signals.

$[8]$ 
can be generated by ambient energy inside the human body just from mechanical vibrations. Other sources of ambient energy have been proposed in literature, such as a Power Generation Photodiode (PGP) [10], body heat, which can produce up to

3.7-6.4 $\mathrm{W}$ of recoverable power, blood pressure, which has approximately $0.93 \mathrm{~W}$ of power available, or limb and body motion, which could potentially power a portable computer [11]. Harnessing even a slight percentage of the power available in the human body can result in the ability to have implantable biosensors with no battery power required.

A block diagram of a typical biomedical transmitter can be seen in Figure 2.2. The first block is the biomedical sensor, which takes a physical property in the human body and converts it to an analog voltage signal. Because this signal is usually at a very low voltage level, a low-noise programmable gain amplifier is the next step for the transmitter. The anti-aliasing filter removes aliasing components in the signal, and then passes it to the analog-to-digital converter (ADC). The ADC converts the analog signal into digital bits and then passes these bits to a digital signal processing (DSP) block. DSP will either store the bits for analysis to be performed at a later time, or it may perform analysis on the signal right away, and then pass it to a wireless transmitter. In order to have a low-power circuit, all components in Figure 2.2 need to be designed with low-power as a priority.

\subsubsection{Wireless Sensor Network}

Low-power ADCs are also an essential component of signal processing with distributed sensors. Distributed sensors are used in a variety of applications and their importance 


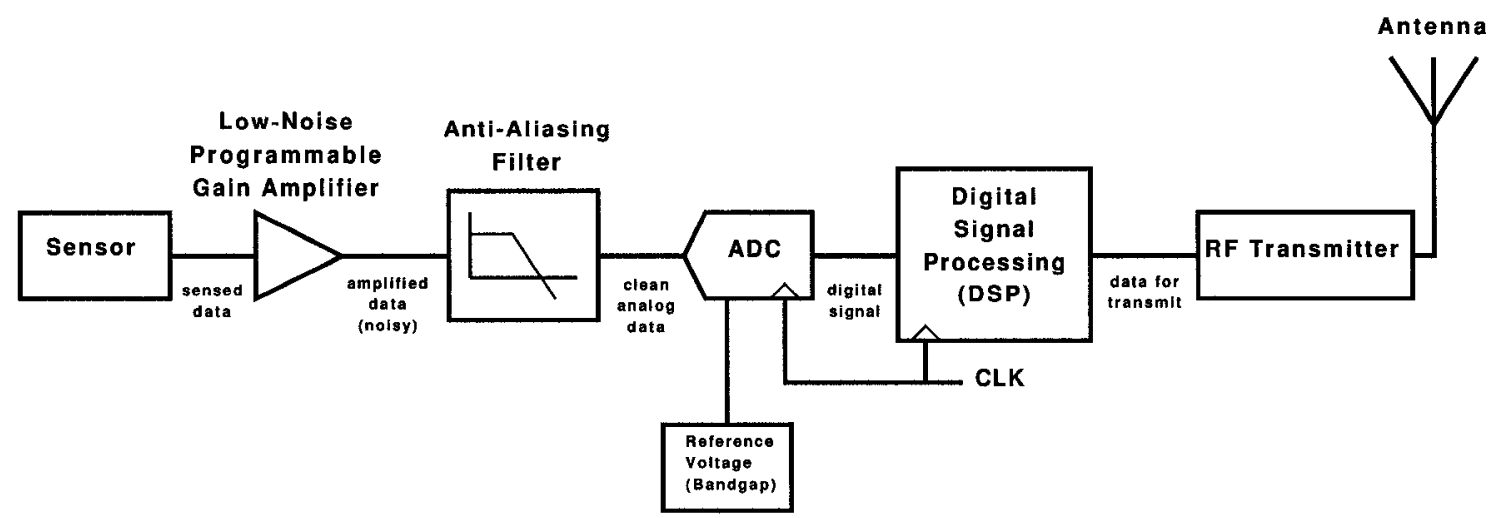

Figure 2.2: Sample block diagram of a biomedical transmitter.

has grown in recent years. Their use has increased due to their relatively low cost, inherent redundancy capabilities with multiple sensors, and the increased computational ability and availability of high speed networks [6]. Classic centralized sensor networks are implemented by having the local sensors communicate all data to a centralized processor, which would perform optimization and data analysis. This scheme is restrictive, as the possibility exists that the coverage area is not fully encompassed by overlapping sensors, and a signal may not be returned from some sensors. The alternative to this scheme is a decentralized scheme, where each sensor performs data processing and minimal data is passed between sensors back to a central processor, or fusion centre [1]. An example of a serial network topology is shown in Figure 2.3. When a "Phenomenon" occurs, each sensor passes quantized information to the next sensor, in series. The sensor generates information based on past observations and the data received from the previous sensor [1].

In order for each sensor to perform data processing, it needs to be equipped with an $\mathrm{ADC}$ in order to convert sensed data to a digital signal that can be processed at the sensor node. The increased tasks required from the sensor increases its need for power. 


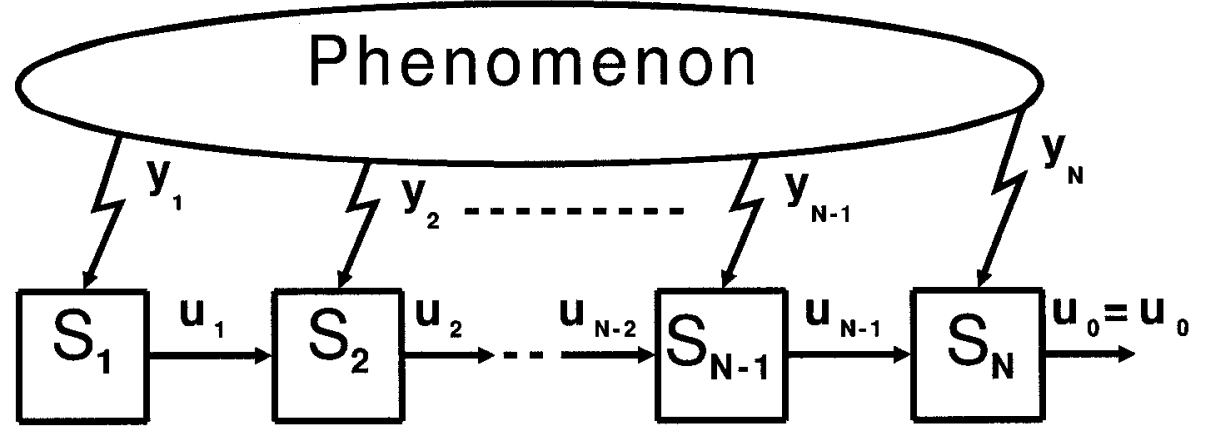

Figure 2.3: Serial topology for a wireless sensor network [1].

Therefore, power management is one of the crucial bottlenecks of the wireless sensor network design. Long-term sensor deployment and self-powered systems are becoming increasingly attractive, as power efficiency of the sensors are improved [12]. Radio receivers require the massive deployment of wireless sensor networks with moderate sample rates, but extreme power constraints, below $15 \mu W[7]$.

\section{$2.2 \quad$ Design Specifications}

The proposed ADC uses a standard $0.13 \mu m$ CMOS process with a nominal supply voltage of $1.2 \mathrm{~V}$. An analysis will be performed to show the ADC functioning at lower voltages, down to $0.4 \mathrm{~V}$. The input for the selected successive approximation $\mathrm{ADC}$ architecture ranges from $\frac{1}{2} V_{D D}$ to $V_{D D}$; therefore, with a $V_{D D}$ of $0.6 \mathrm{~V}$, the analog input needs to be between $0.3 \mathrm{~V}$ to $0.6 \mathrm{~V}$. The main target of this $\mathrm{ADC}$ is biomedical and wireless sensor network applications, which require low-to-medium sample rates, meaning sample rates of approximately $1 \mathrm{kS} / \mathrm{s}$ to $1 \mathrm{MS} / \mathrm{s}$. The resolution required for biomedical devices is 8 to 10 bits, such as those found in [13-21]. In order to digitize an EEG signal, there are two main approaches: the first approach is the digitize the EEG signal and the drift signal at the same time, and then remove the drift 
digitally, which would require 20-bit resolution [22]. The second approach is to filter the drift before conversion, relaxing the ADC resolution requirement to 10 -bits [22]. The presented architecture provides 10-bit resolution.

Power dissipation is the most important design concern for the presented ADC. A power dissipation of $2 \mu W$ will be targeted to be competitive with published literature. In order to achieve low power dissipation, the ADC will be designed to operate at low voltage levels, forcing the CMOS transistors into the subthreshold region of operation. This will be accomplished by applying a supply voltage of $0.6 \mathrm{~V}$ to the ADC, resulting in the input range of the $\mathrm{ADC}$ to be between $0.3 \mathrm{~V}$ and $0.6 \mathrm{~V}$. The resolution is to be designed for 10 bits, as most published ADCs with similar applications have 8-12 bits resolution. The binary weighted capacitor array design is expected to require most of the ADC area. After including the electrostatic discharge (EDS) protection, and pads for the input and output pins, the dimensions of the chip will be targeted to a maximum of $1.25 \mathrm{~mm} \times 1.25 \mathrm{~mm}$. The goal of the ADC is to operate with no missing codes and an Integral Non-linearity (INL) and Differential Non-linearity (DNL) below 1 LSB. The focus of the design is to obtain minimum power dissipation with some trade-offs on accuracy and speed, and minimal trade-offs on area.

\subsection{Summary of ADC Specifications}

The targeted applications for the ADC is an implantable biomedical sensor, which is essential in analyzing biomedical signals in the human body, such as EOG, EEG, ECG, EMG, and AAP, and wireless sensor networks. The applications require an ADC with low power dissipation, which can be used on signals with frequencies in 
the $\mathrm{kHz}$ range. The required design is a successive approximation register ADC with 10-bit resolution, operating at a $V_{D D}$ of $0.6 \mathrm{~V}$. A power dissipation under $2 \mu \mathrm{W}$ and an area under $1.5 \mathrm{~mm}^{2}$ are targeted. The design specifications have been summarized in Table 2.1.

Table 2.1: Summary of the desired specifications for the proposed ADC.

\begin{tabular}{|c|c|}
\hline Design Parameter & ADC Specification \\
\hline \hline ADC Architecture & Successive approximation \\
\hline Technology & $0.13 \mu \mathrm{m}$ CMOS \\
\hline Resolution & 10 -bit \\
\hline Source Voltage, $V_{D D}$ & $0.6 \mathrm{~V}$ \\
\hline Input Range & $0.3 \mathrm{~V}-0.6 \mathrm{~V}$ \\
\hline Sample rate & $1 \mathrm{kS} / \mathrm{s}-1 \mathrm{MS} / \mathrm{s}$ \\
\hline INL & $<1 \mathrm{LSB}$ \\
\hline DNL & $<1 \mathrm{LSB}$ \\
\hline Power & $<2 \mu W$ \\
\hline Area & $<1.5 \mathrm{~mm}^{2}$ \\
\hline
\end{tabular}




\section{Chapter 3}

\section{Background}

\subsection{ADC Architectures}

In order to ensure the correct $\mathrm{ADC}$ architecture is chosen for the desired low-power, medium-speed requirement, an analysis of popular architectures was performed, including a Flash ADC, a Pipeline ADC, an Integrating ADC, a Sigma Delta $(\Sigma \Delta)$ ADC, and a Successive Approximation ADC.

\subsubsection{Flash ADC}

Flash ADCs allow for the fastest conversion from an analog signal to digital. Flash ADCs may work on a system of thermometer code encoding with cascading high-speed comparators. Thermometer code encoding employs $\left(2^{n}-1\right)$ comparators, along with $2^{n}$ resistors, for an n-bit converter. The resistors act as a multi-level voltage divider, providing a different reference voltage to each comparator. The reference voltage of a comparator is one LSB voltage less than the one above it, and the input offset for each comparator is smaller than the LSB of the entire ADC. The block diagram of a typical N-bit flash ADC can be seen in Figure 3.1.

The thermometer code is determined by examining how many reference voltages 


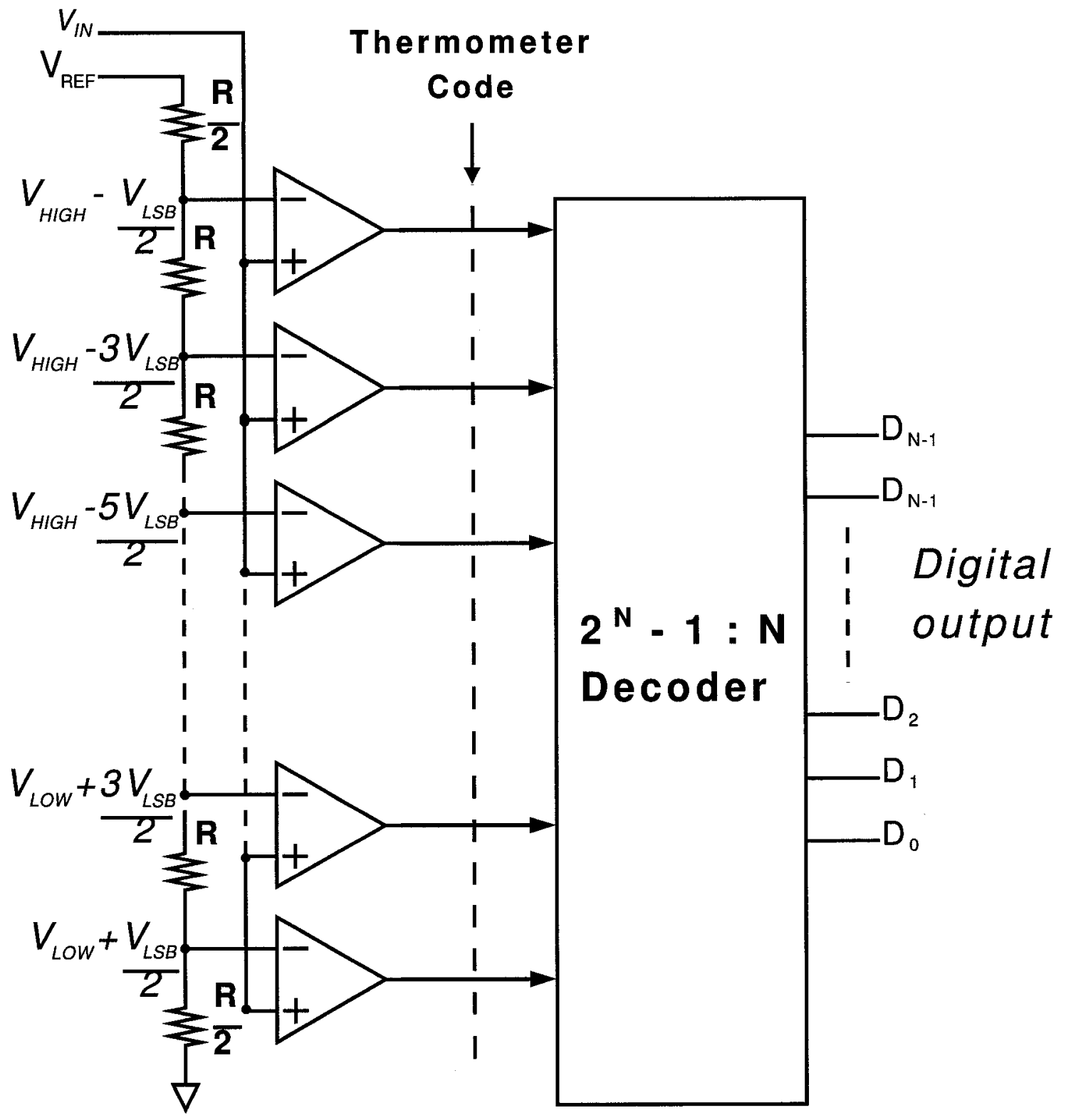

Figure 3.1: Block diagram of a Flash ADC. 
are higher than the analog input, and how many are lower. The comparators work in parallel, and if the input is higher than the reference voltage of a specific converter, a digital ' 1 ' is passed to the decoder, while if the input is lower than the reference voltage, a digital ' 0 ' is passed to the decoder. For a 10-bit converter, 1023 bits are passed to the decoder in parallel. This means that 1023 converters need to perform simultaneously, which requires a large power dissipation and a large chip area. With each bit of resolution increase, the chip area and power are almost doubled [23]. For this reason, the Flash ADC is usually limited to 8-bit resolution. The accuracy of the Flash ADC depends largely on the ability to match the resistors in the voltage divider network, as is shown in the equations for INL and DNL, from [24]:

$$
\begin{gathered}
|I N L|_{\text {max }}=\frac{V_{R E F}}{2} \cdot\left|\frac{\triangle R_{k}}{R}\right|_{\text {max }}+\left|V_{o s, i}\right|_{\max }, \\
|D N L|_{\max }=\frac{V_{R E F}}{2^{N}} \cdot\left|\frac{\triangle R_{i}}{R}\right|_{\max }+2\left|V_{o s, i}\right|_{\max } .
\end{gathered}
$$

We can see from 3.1 and 3.2 that both INL and DNL depend on the resistor variation from processing and silicon doping variations, $\triangle R$. Resistors are inherently susceptible to large variation, but in order to have an accurate ADC and resistor network, it needs to be ensured that the resistors are properly matched, otherwise, the errors in the resistors will translate to errors in conversion. Matching the resistors to ensure that less than one quantization level is lost due to resistor mismatch results in the ADC operating at the desired resolution. During the processing steps required to create a physical circuit on the wafer, non-idealities often occur, such as lateral 
diffusion, etching under the protective layer, and three-dimensional etch effects. These processing non-idealities may cause a large variation between the layout of a resistor and its subsequent processed on-chip value.

Some methods for reducing power consumption in Flash ADCs involves reducing power requirements for the actual comparators [25], using a lower number of comparators [26], or selectively turning on the required comparators depending on the voltage level of the analog input [27]. However, from observing current low-power Flash ADCs none of these methods can provide an architecture with power consumption low enough to meet the desired specifications.

\subsubsection{Pipeline ADC}

A pipeline $\mathrm{ADC}$ is an $\mathrm{N}$-step converter, where 1 bit is converted per stage and the stages are in series. It is able to reach high resolutions (10-13 bits) at relatively fast speeds. Each stage of a pipeline ADC contains a comparator, a sample-and-hold circuit, a summer, and an amplifier with a gain of 2 . The block diagram for a typical N-bit pipeline ADC can be seen in Figure 3.2. The comparator works such that an input signal is compared to $\frac{V_{R E F}}{2}$, and if the input is greater than $\frac{V_{R E F}}{2}$, a digital 1 is output from the comparator. After this first step, $\frac{V_{R E F}}{2}$ is subtracted from the original input signal and the result is passed to the amplifier with a gain of 2 . If the input is smaller than $\frac{V_{R E F}}{2}$, then the output of the comparator is a digital 0 and the original input is then passed through to the amplifier with a gain of 2 . The amplifier multiplies the result by 2 and the result is passed to the sample-and-hold circuit of the next stage. 
It has been shown in [28] that it is possible to perform conversions using a pipeline ADC at a rate higher than 1 bit per cycle using switched buffer and switched operational amplifier design techniques. This design resolves 1.5 bits per stage, where 0.5 bits are used for digital error correction, with a redundant signed digit (RSD) algorithm.

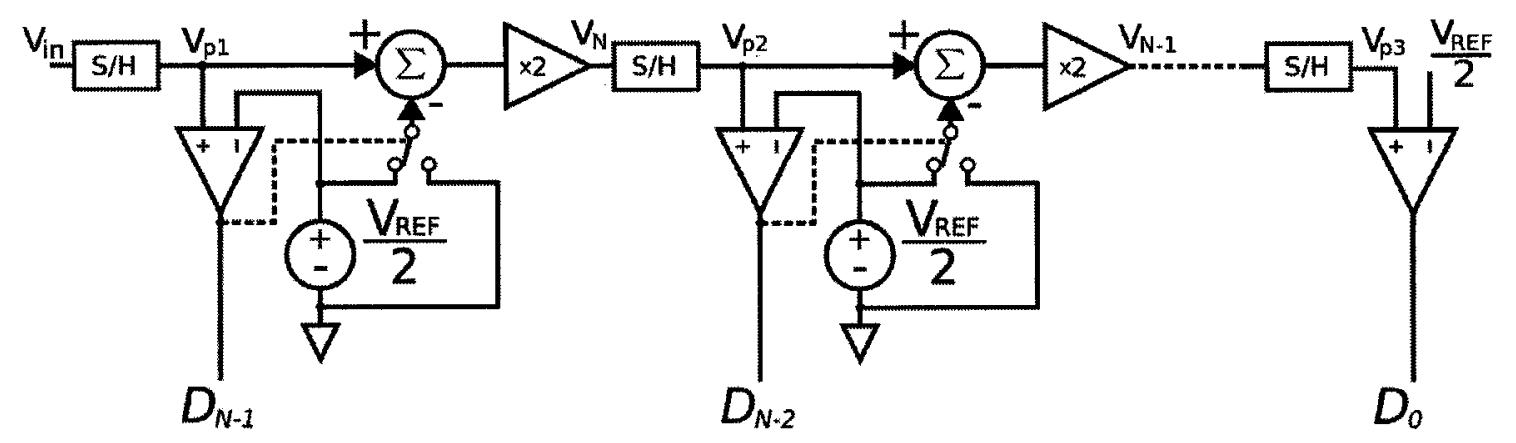

Figure 3.2: Block diagram of a Pipeline ADC.

The pipeline converter can give high throughput, as it completes a conversion in one cycle; however there is an N-cycle delay before the initial output is seen, for an N-bit converter. For a 10-bit converter, the pipeline ADC would require 10 converters. However, the first stage conversion requires the highest accuracy, while subsequent stages can implement less accurate, and less power-hungry designs, if power consumption is a concern. Overall, the Pipeline ADC operates at high speeds using a lower layout area and with lower power dissipation than the Flash ADC; however, the power required for the comparator, sample-and-hold circuit, amplifier, and adder for every stage does not make it the ideal candidate for a low-power ADC application. 


\subsubsection{Integrating ADC}

The integrating ADC integrates the input signal in order to correlate it with a digital counter. These converters are generally useful in applications that require high resolution, but that do not require fast conversion rates. The ramp voltage is compared to the analog input by comparing every step of the ramp to the input. A counter is required to count the number of cycles required for the analog input to match a desired voltage level at the digital ramp. Once the input matches the ramp, the number of clock cycles that have passed since the comparison cycle has started determines the digital representation of the analog input. An integrating ADC consists of an integrator, to generate a ramp voltage, a counter, and a comparator. There are two types of Integrating ADCs, using Single-Slope and Double-Slope architectures.

\section{Single-Slope Architecture}

The single-slope architecture has an integrator, which integrates the reference voltage, and one counter, which determines the number of clock cycles required before the integrated value of the reference voltage is equal to the sampled input. A block diagram of the single-slope integrating ADC architecture can be seen in Figure 3.3. The output of the counter is the digital representation of the analog voltage since the number of clock cycles required is proportional to the analog input's location between $V_{l o w}$ and $V_{D D}$. An N-bit integrating ADC's counter might have to increment up to $2^{N}$ clock cycles if the analog input is close to $V_{D D}$. If the analog input is low, fewer clock cycles are required. Since input values that are close to $V_{D D}$ require many clock cycles, this becomes a comparatively slow architecture. For example, a 10-bit ADC, 
sampled at $100 \mathrm{kS} / \mathrm{s}$ would require a $102.4 \mathrm{MHz}$ clock, which could potentially lead to higher power dissipation, and is not desirable when low-power operation is essential to the design.

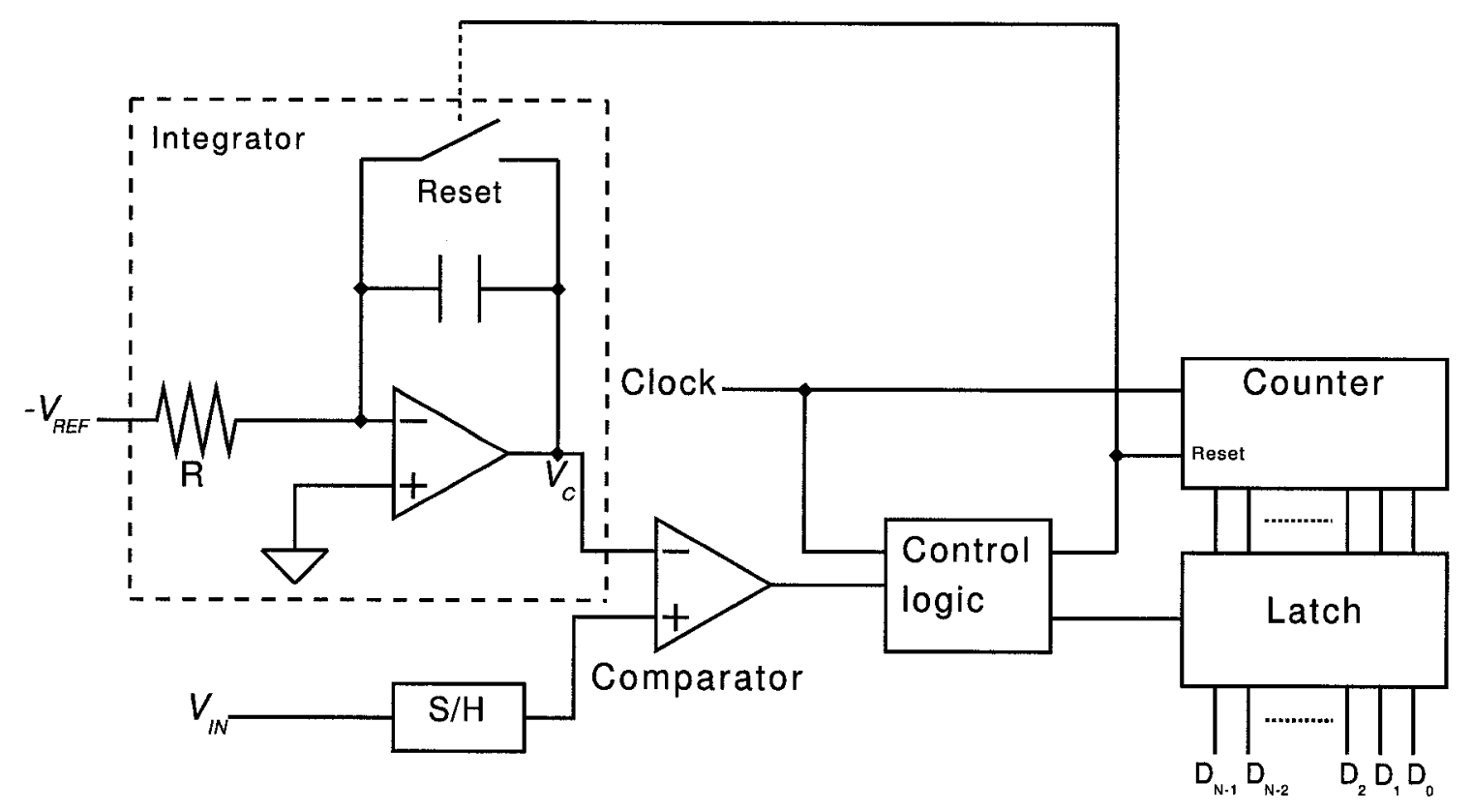

Figure 3.3: Block diagram of a single-slope Integrating ADC.

\section{Dual-Slope Architecture}

Dual-slope architecture has one integrator that performs two integrations. The initial step is the integration of the negative input signal, which has gone through the sampleand-hold circuitry. A block diagram of the dual-slope integrating ADC architecture can be seen in Figure 3.4. This integration results in a positive slope with a fixed length, dictated by the counter. When the counter overflows and resets, the input of the integrator is connected to the positive reference voltage, and a second, negative slope, is achieved, ultimately resulting in a discharge of the initial slope down to zero, at a constant rate. A counter is used to measure the amount of time it took for the integration to discharge down to zero, generating the digital output. The dual-slope 
architecture suffers drawbacks similar to the single-slope integrating ADC. In order to achieve sample rates in the order of hundreds of $\mathrm{kS} / \mathrm{s}$, a fast clock is required, thereby increasing the power of the overall circuit. A $62 \mu W$ dual-slope integrating ADC in [5] showed promise as a low-power design; however, the ADC was limited to sample rates of $800 \mathrm{~S} / \mathrm{s}$, and worked from a $1.6 \mathrm{MHz}$ clock.

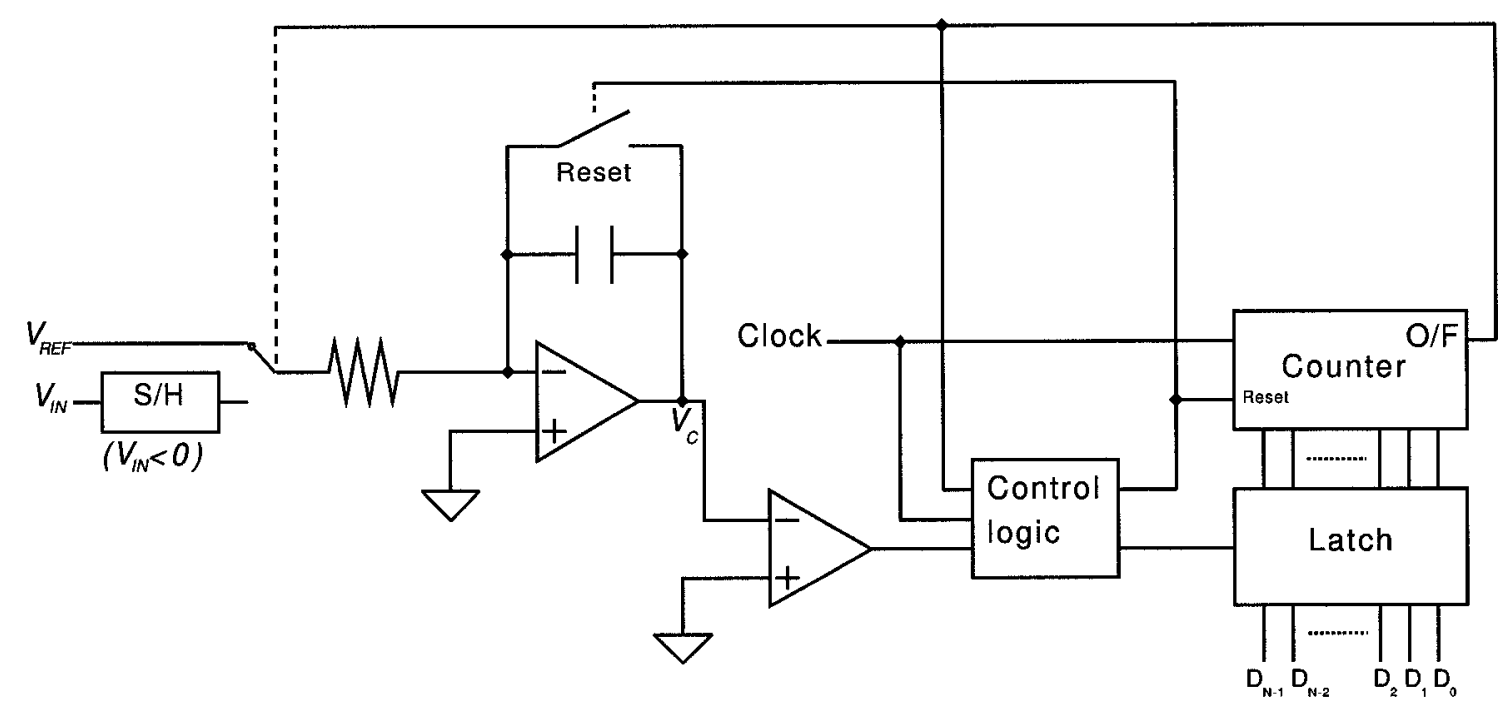

Figure 3.4: Block diagram of a dual-slope Integrating ADC.

\subsubsection{Sigma-Delta $(\Sigma \Delta)$ ADC}

The ADCs that have been mentioned previously sample the input at the Nyquist rate, $f_{N}=2 F$, where $F$ is the bandwidth of the signal, and $f_{N}$ is the sampling rate. The Nyquist rate is the lowest sampling rate that permits an accurate reconstruction of the analog input signal. There is another type of converter, which samples the input signal at rates much higher than the signal bandwidth; the oversampling, or Sigma-Delta, ADC.

The general approach used by a sigma-delta $\mathrm{ADC}$ is to find the quantization error between the oversampled analog input and the output of the digital to analog 


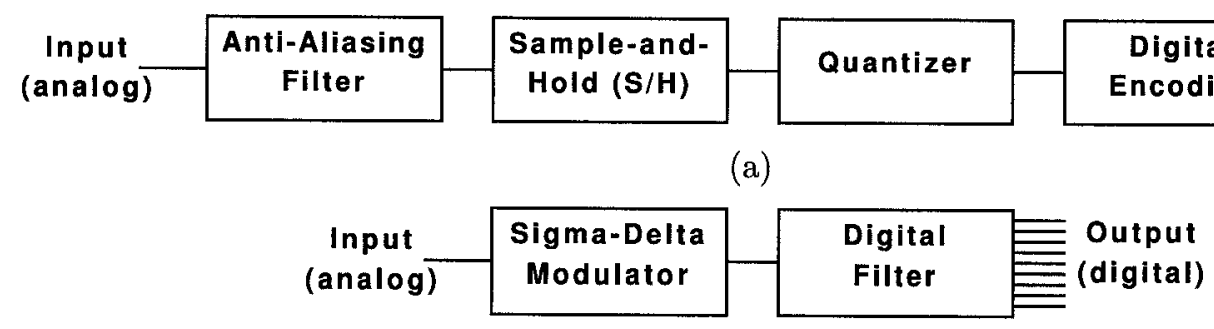

(b)

Figure 3.5: Typical block diagrams for the (a) Nyquist rate ADC and (b) Oversampling ADC.

converter (DAC). The complexity of the design is reduced by the 1-bit ADC, thereby reducing the overall complexity of the design and the amount of chip area required. This type of $\mathrm{ADC}$ does require a fast clocking and filtering mechanism, however, which can negate potential power saving attempted in order to make it suitable for lowpower biomedical applications. An advantage of the sigma-delta architecture is that the modulator creates the necessary pulses in real-time, avoiding the need to store the input for any period of time and removing the sample-and-hold circuit entirely. Figure 3.6 (a) shows the block diagram of a first-order sigma-delta modulator, which uses an integrator, a 1-bit ADC, and a 1-bit DAC in a single-feedback loop. The singleloop architecture is the preferred method of $\Sigma \Delta$ design if power consumption is a concern $[29,30]$. A sampling frequency of $50 \mathrm{kHz}$ was achieved at a power consumption of $78 \mu \mathrm{W}$ using this method [29]. However, for biomedical applications, an even lower power dissipation is desired. A higher order architecture is shown in Figure 3.6 (b).

\subsubsection{Successive Approximation ADC}

The successive approximation ADC finds the output by performing a binary search on the analog input through possible quantization levels before reaching a final digital 


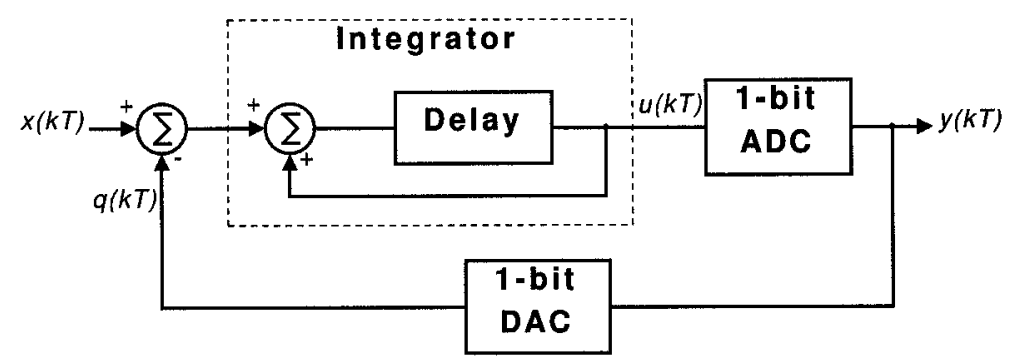

(a) First-order sigma-delta modulator.

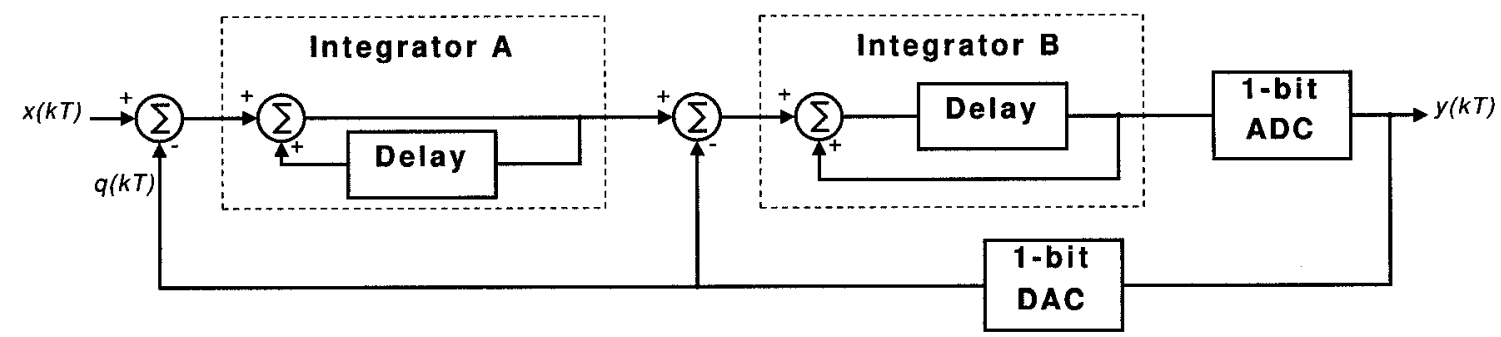

(b) Second-order sigma-delta modulator.

Figure 3.6: Block diagrams of two sigma-delta modulators.

output. A block diagram of the successive approximation ADC can be seen in Figure 3.7(a). A sampled version of the input, $V_{I N}$, is compared to the output of the DAC, and the result controls the direction of the binary search, as well as the output of the successive approximation register (SAR), which is the digital output of the ADC. Figure 3.7(b) shows the process of determining the digital output using a successive approximation ADC, with a binary search. The initial input is sampled and compared to the middle value of the input analog range, meaning that if the range is $-V_{D D}$ to $V_{D D}$, the input is compared to $0 \mathrm{~V}$. If the analog input is greater than 0 , then a 1 is sent to the most significant digital output bit, and the comparison process is continued. The next step would involve comparing the sampled input to the value in the middle of $0 V$ and $V_{D D}$, or $\frac{V_{D D}}{2}$. Similarly, if the input is shown to be below $0 V$, a digital 0 is sent to the most significant output bit, while the next step involves comparing the input to the value between $-V_{D D}$ and $0 V$, or $-\frac{V_{D D}}{2}$. This process of comparison narrows the 
input down to a small range of voltages and the appropriate digital representation is what remains. Successive approximation analog to digital converters are amongst the most popular approaches to performing A/D conversions due to their ability to have relatively quick conversion time, while maintaining moderate circuit complexity [31]. They have also been shown to be useful for biomedical applications where high speed is not a requirement, as power consumption needs to be reduced [18].

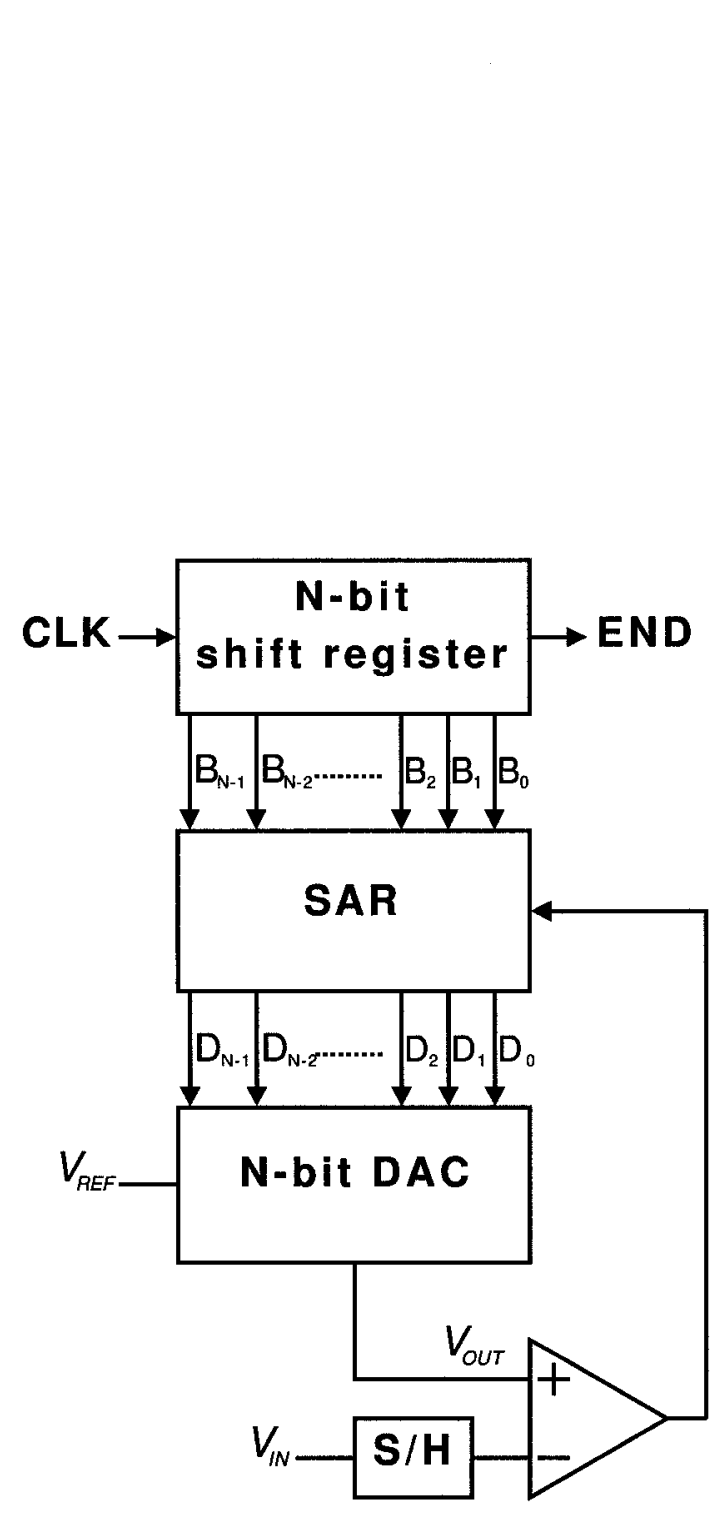

(a) Block diagram of the SAR ADC.

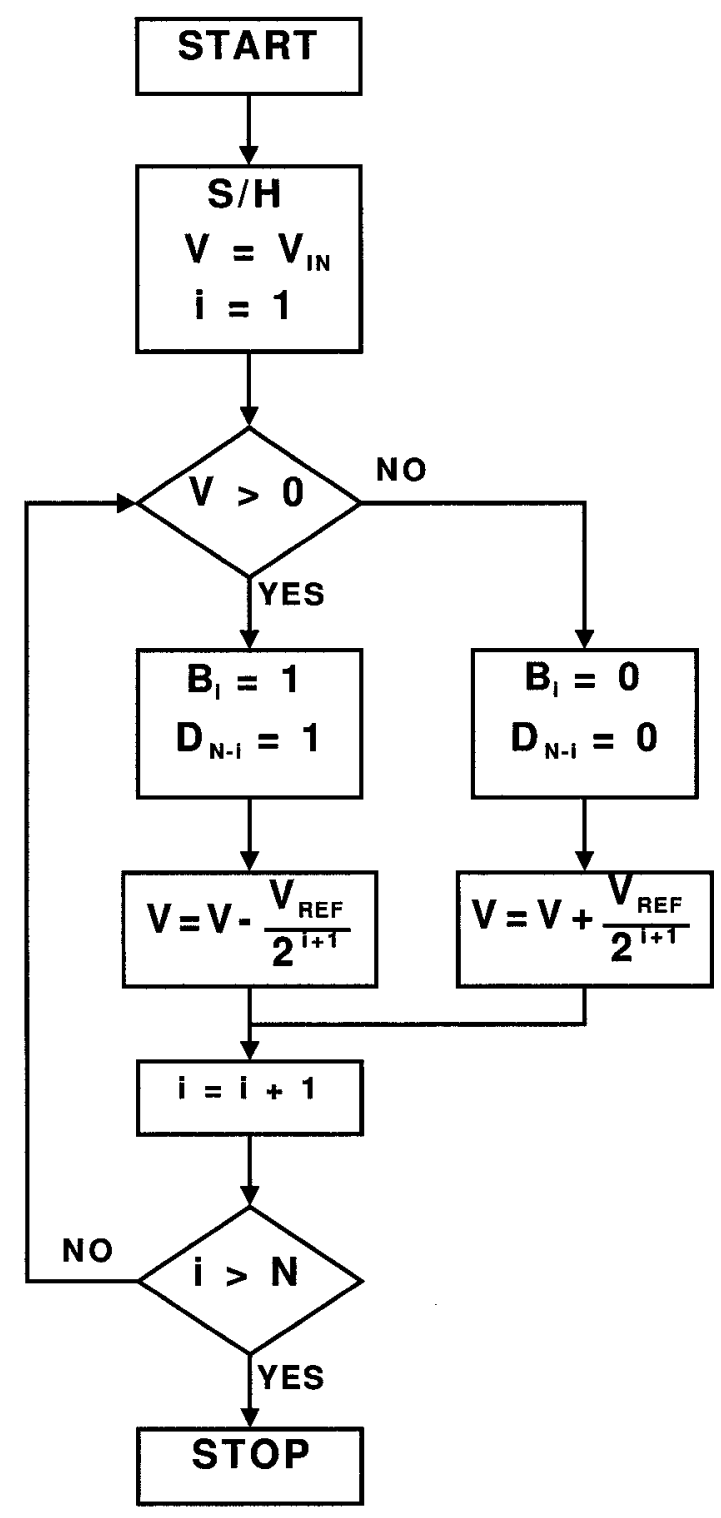

(b) Flow chart for the SAR ADC.

Figure 3.7: Successive approximation register ADC block diagram and flow chart. 


\subsection{Low-Power Design Architectures}

Table 3.1 shows a list of published measured low-power ADC designs. It is evident that SAR, or successive approximation register, ADCs give the best low-power performance. The only SAR ADC that uses a $0.09 \mu \mathrm{m}$ process is observed in [32]. The presented design uses sub-threshold analog circuit design techniques in order to achieve low power. The design shown by [33] also uses the sub-threshold regions of the MOSFET to design its analog circuitry as part of a pipeline ADC. The comparator used in this design consists of two inverters cascaded in series, and is designed to operate in the sub-threshold regime. We can see that the power dissipation of this 6-bit Pipeline ADC is higher than the 6-bit SAR ADC shown by [32].

The ADC presented in [13] achieves low energy consumption, down to $31 \mathrm{pJ} /$ cycle, using a successive approximation ADC with charge redistribution. The design uses the supply voltage as a reference voltage in order to avoid an on-chip bandgap circuit, which would increase the power requirement. It also does not include offsetcancellation techniques in order to decrease power consumption and it uses a microprocessor to do single sample offset calibration. The ADC design shown in [14] uses a similar architecture, while attempting to bring $\mathrm{V}_{D D}$ down to $0.5 \mathrm{~V}$; however, the design uses a sample-and-hold circuit that is not integrated on-chip. The design in [15], as well as [17], uses an attenuation capacitor in order to reduce the size of the capacitors required in the capacitor array. [17] uses a silicon-on-sapphire $0.5 \mu m$ process, while achieving a power dissipation of $7.3 \mu \mathrm{W}$. The low-power integrating ADC presented in [16] uses a novel architecture, which uses a series of successive 
integrate-and-fire operations, in combination with sub-threshold design techniques to achieve low power dissipation. The two successive approximation ADC designs shown in [18] have among the lowest power consumptions. These designs use a successive approximation architecture with an attenuation capacitor, which is designed for tunability with a 3-bit digital input. Subthreshold techniques are implemented for the analog and digital parts of the circuitry. One of the designs uses a full swing input, while the second is limited to a swing of $\frac{1}{2} V_{D D}$.

The $\Sigma \Delta \mathrm{ADC}$ presented in [19] has a low power dissipation of $108 \mu \mathrm{W}$, however the architecture used is undesirable, since it uses additional clocking and the input is oversampled at a higher rate than the Nyquist frequency. Architectures other than the $\Sigma \Delta$ are able to perform at a lower power dissipation, such as the integrating ADC design from [20], which uses a BiCMOS process and low sample rates in order to achieve a low power dissipation. However, its energy per cycle is $6.2 \mathrm{~nJ} /$ cycle, which is well above other published works. The design for the $32.6 \mu W \mathrm{ADC}$ shown in [21] is the ADC with the lowest published power dissipation for a 10-bit SAR architecture. The architecture uses a charge-recycling method to select the capacitors that are being used during conversion. The lowest power for a 10-bit ADC is noted in [20], where $17.9 \mu \mathrm{W}$ is dissipated. This power is noted at a sample rate of $2.9 \mathrm{kS} / \mathrm{s}$, which is lower than desired for the intended application. The 16-bit successive approximation ADC shown in [34] has a low power dissipation, but the low sample rate of $200 \mathrm{~S} / \mathrm{s}$ causes the energy per cycle to be high, at $110 \mathrm{~nJ} / \mathrm{cycle}$. This design implements an architecture which allows for a power consumption of less than $1 \mu W$ when the ADC is in standby mode. 


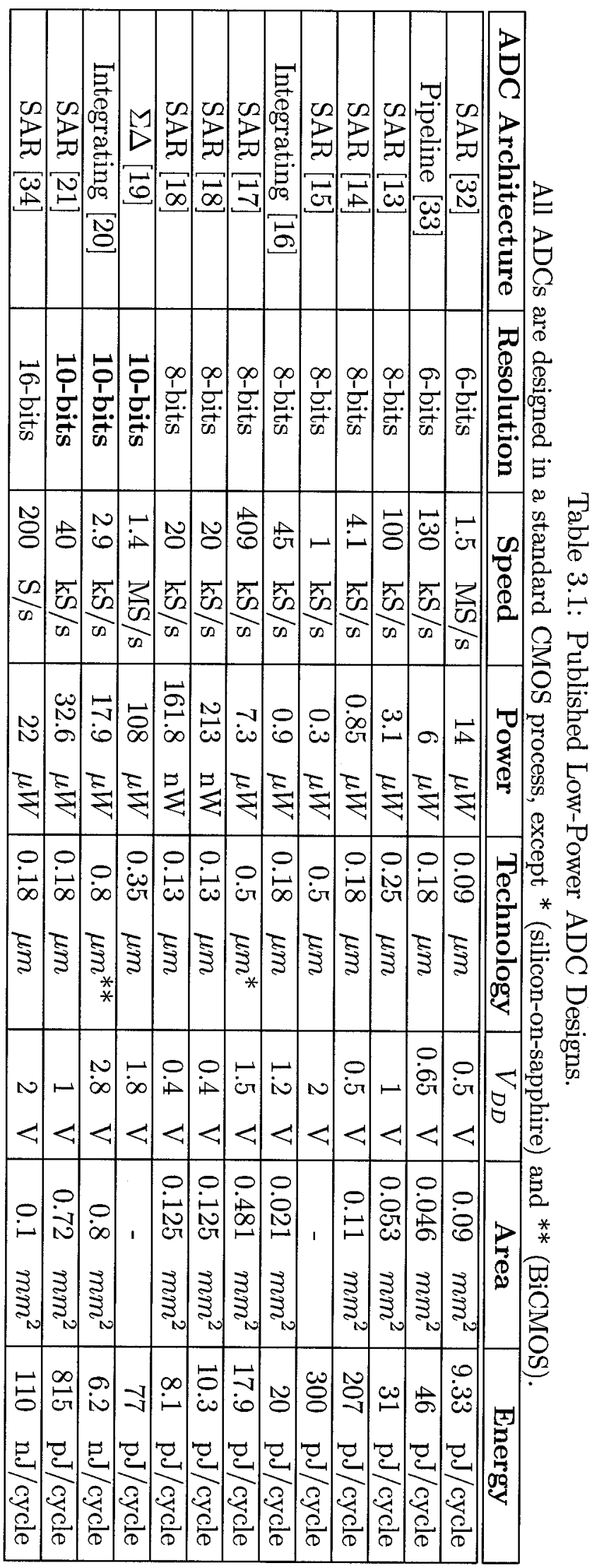




\subsection{Background Summary}

In order to decide the ADC architecture that best meets the specifications laid out in Table 2.1, an analysis of the common ADC architectures was performed. Flash ADC, Pipeline ADC, Integrating ADC, Sigma-Delta ADC, and Successive Approximation ADC architectures were analyzed. It was found that a Flash ADC with a 10-bit resolution would require 1023 converters to decode 1023 bits in parallel. These converters require too much power to meet the desired power specification. The pipeline ADC requires a converter, a sample-and-hold circuit, an amplifier, and an adder for each bit resolution, making the power requirement too high to meet the desired specification. The integrating ADC requires a very fast clock that can lead to the need for high power dissipation. In order to achieve sample rates in the order of hundreds $\mathrm{kS} / \mathrm{s}$, a clock in the order of hundreds $\mathrm{MHz}$ is required. The Sigma-Delta ADC requires fast clocking and a filtering algorithm, which increase its power requirements beyond the specification. The SAR ADC was chosen because it requires only one converter and allows for low-power designs when high speed is not essential.

A summary of published low-power ADC designs is given in Table 3.1 and it shows that the SAR architecture is the most common for applications requiring low power when high speed is not a major concern. 


\section{Chapter 4}

\section{ADC Circuit Architecture}

\subsection{Successive Approximation ADC Performance}

In Chapter 3, various $\mathrm{ADC}$ architectures were analyzed, and it was shown that for a low-power $\mathrm{ADC}$, the desired architectures are the integrating $\mathrm{ADC}$, the sigma-delta $(\Sigma \Delta) \mathrm{ADC}$, and the successive approximation ADC. However, the $\Sigma \Delta \mathrm{ADC}$ requires oversampling, which leads to higher power requirements for the digital circuitry, while the integrating $\mathrm{ADC}$ is limited to low sample rates, due to the requirement of a clock at a $2^{N}$ frequency for an N-bit ADC. This leaves the successive approximation ADC as the remaining choice for a low-power design, to be used in biomedical and wireless sensor applications. The architecture used for the presented successive approximation ADC is based on charge redistribution in a sampling binary weighted capacitor array, similar to that presented in [35]. Other similar low power SAR ADCs were demonstrated in [13-15]. The standard SAR architecture includes a sample-and-hold circuit, a capacitor array, a comparator, a DAC, and digital control logic. Since the intention of the presented $\mathrm{ADC}$ is operation in low-power applications, an architecture that does not require a sample-and-hold circuit is selected, thereby requiring a 
comparator, a capacitor array, low-power CMOS digital logic, and analog switches. The presented SAR ADC has an input range from $\frac{1}{2}$ VDD to VDD. The main phases of the conversion cycle are similar to those described in $[35,36]$ :

(i) Analog data, $V_{i n}$, is sampled onto the bottom plate of the capacitor array, while the top plate is set to $\frac{1}{2} V_{D D}$, as shown in Figure 4.1. This results in a voltage across the capacitor array of $\frac{1}{2} V_{D D}-V_{i n}$.

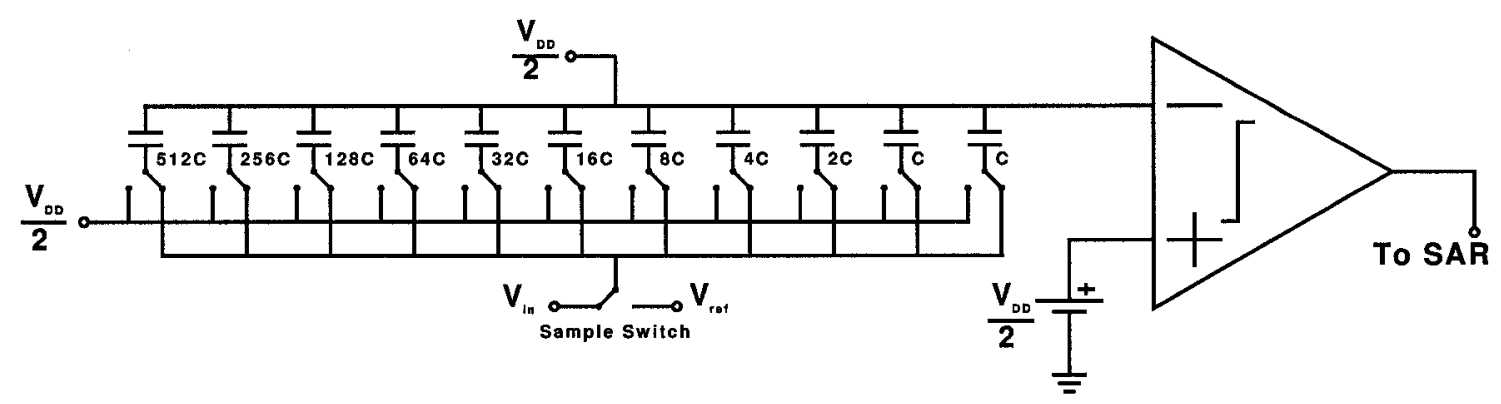

Figure 4.1: Sampling phase of the SAR ADC.

(ii) The bottom plate of the capacitor array is forced to $\frac{1}{2} V_{D D}$, causing the top plate to become $V_{D D}-V_{i n}$, since the charge across the capacitor remains static. This step can be seen in Figure 4.2.

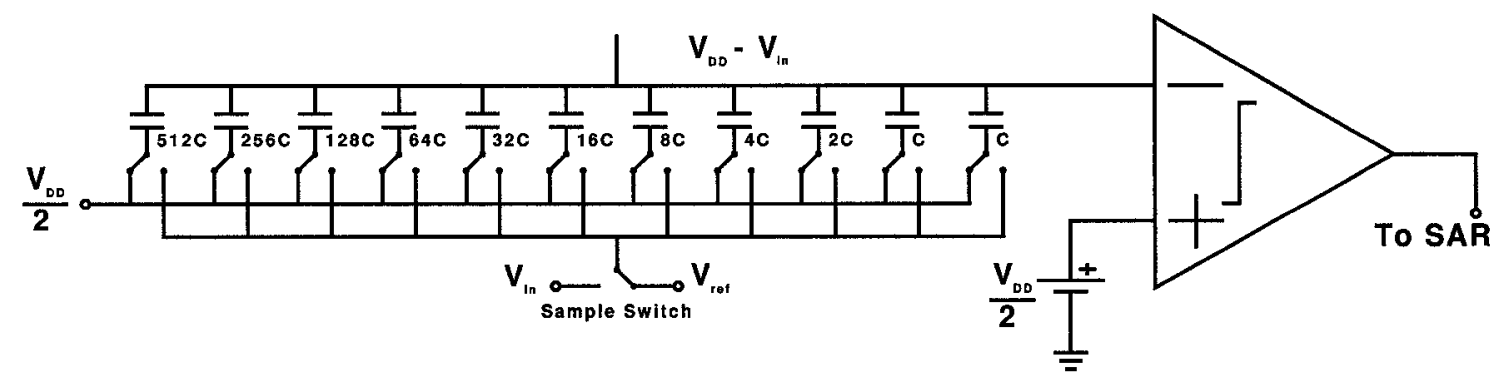

Figure 4.2: Sampling phase of the SAR ADC during which the top plate of the capacitor becomes $V_{D D}-V_{i n}$.

(iii) The next step is the approximation stage, as shown in Figure 4.3. This is where the digital SAR logic controls the switching, starting with the most significant 
bit (MSB), and working down to the least significant bit (LSB). The comparator determines if the voltage on top of the plate of the capacitor array is higher or lower than $\frac{1}{2}$ VDD and outputs a digital 0 or 1 respectively. A full $n$-bit conversion will require $\mathrm{n}$ clock cycles, meaning that for the 10-bit SAR ADC required, 10 clock cycles are used for this step. Figure 4.4 shows the voltage on the top plate, and the location of the capacitor switches when the analog input leads to a digital output of '1001100100'.

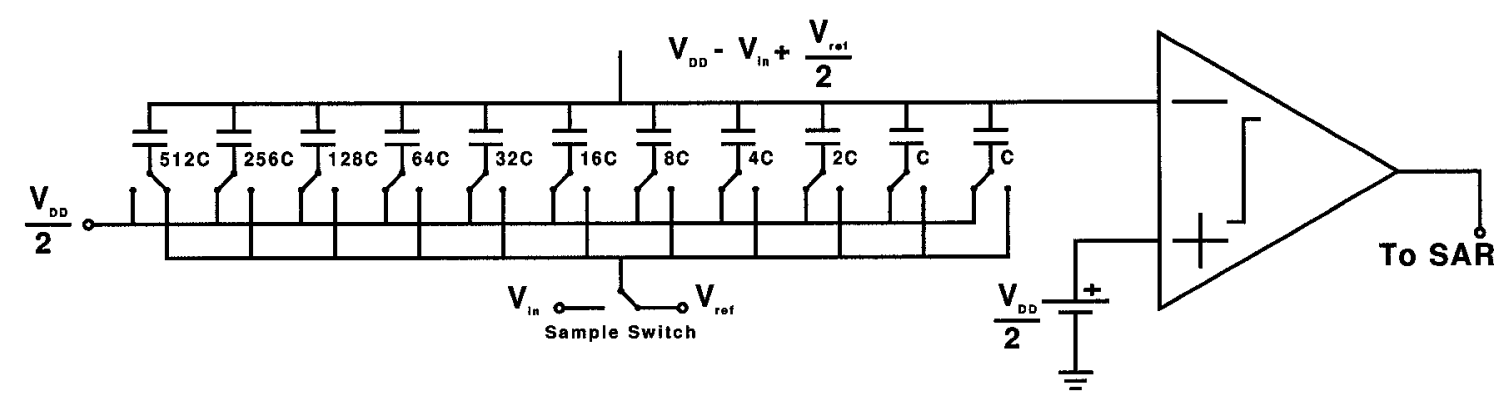

Figure 4.3: Approximation phase of the SAR ADC, with the MSB approximation step shown

(iv) After completing the approximation for the least significant bit, a new conversion cycle begins, and the previous data is valid. An extra four clock cycles are required to sample the analog signal, force it negative, and output the digital data after it is found to be valid, resulting in a total of $n+4$ clock cycles for approximating one n-bit cycle.

\subsection{ADC Components}

The architecture of the chosen successive approximation ADC with charge redistribution contains three main components (SAR, comparator, and capacitor array), switches, and buffers. Their functions will be described briefly in this section, while 


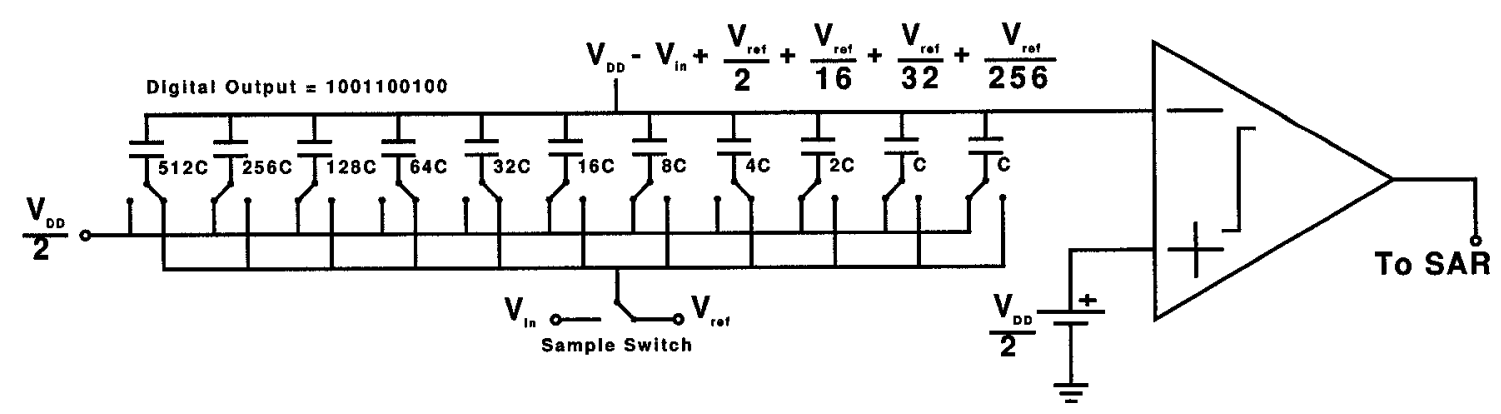

Figure 4.4: Approximation phase of the SAR ADC, with the MSB approximation step shown

their detailed functions and topologies in the designed ADC will be further described in Chapter 5.

\subsubsection{Digital Successive Approximation Register (SAR)}

The successive approximation register is the main digital component of the successive approximation analog-to-digital converter (ADC). The output of the SAR is the actual digital output of the ADC, when the data is valid. The register is reset before each conversion, causing all bits to be at a digital ' 0 '. Following the reset, the bits are set to a digital ' 1 ', starting with the most significant bit (MSB) and ending with the least significant bit (LSB). The digital '1' causes the capacitor switch, shown in capacitor '512C' from Figure 4.3 to switch from $\frac{1}{2} V_{D D}$ to $V_{\text {ref }}$, causing the initial addition of $\frac{V_{r e f}}{2}$ to the top plate of the capacitor. If, after the addition, the top plate of the capacitor is lower than $\frac{1}{2} V_{D D}$, the MSB stays at a digital ' 1 ', while if it is higher than $\frac{1}{2} V_{D D}$, it is brought back to a digital ' 0 '. This process is how the SAR determines the digital representation of the analog input. The SAR is implemented with registers and digital gates. To avoid introduction of switching noise to the analog circuitry, a separate power supply was used for the digital components of the chip. 


\subsubsection{Comparator}

The comparator determines whether the top plate of the capacitor array is higher or

lower than the analog ground, $\frac{1}{2} V_{D D}$. In order for the 10-bit ADC to work at voltages down to $0.6 \mathrm{~V}$, the minimum offset voltage of the comparator needs to be at most $\frac{0.3}{2^{10}} V$, or $293 \mu V$. For the successive approximation ADC architecture, a comparator with a desired accuracy of at least $293 \mu \mathrm{V}$ is expected to dissipate most of the power in the ADC. This can be reduced by taking advantage of the sub-threshold regime of the CMOS devices, as described in Section A.1.

\subsubsection{Capacitor Array}

The binary-weighted capacitor array allows the charge redistribution algorithm to be used in the successive approximation ADC architecture. The stored charge across the capacitor acts as the sample-and-hold circuit, and allows for the addition and subtraction of voltages to take place on the top plate of the capacitor array. From Figures 4.1 to 4.4 , the largest capacitor is $512 \mathrm{C}$, while the smallest is C. If the minimum capacitor value is set to $200 \mathrm{fF}$, the largest capacitor would have to be $102 \mathrm{pF}$. This capacitor size would require an area of approximately $0.05 \mathrm{~mm}^{2}$, while requiring large power dissipation to charge and discharge. During conversion, the capacitors in the array get charged and discharged. The size of the capacitors directly influences how much charge is required in order to obtain a desired voltage level on the capacitor array. Having large capacitors increases the energy requirement for the ADC, as well as the time required to charge the capacitors. This means that the speed of conversion is also limited with increasing capacitor sizes. In order to avoid the need for 
such large capacitors, a series attenuation capacitor is used $[15,17,18]$. The value of a metal-insulator-metal (MIM) capacitor, manufactured in the CMOS $0.13 \mu m$ process can experience processing variation of up to $10 \%$ [37]. Matching the capacitors in the array is essential in preventing a degrading performance of the ADC due to capacitor mismatch [24].

\subsubsection{Switching Network for the Capacitor Array}

The switches in the capacitor array switching network need to be able to switch the voltage on the bottom of the capacitors between analog ground $\left(\frac{1}{2} V_{D D}\right)$, and $V_{r e f}$. Since the transmitted signal will always be between $G N D$ and $V_{D D}$, analog switches are required, which act as $\mathrm{RC}$ circuits while transmitting the input signal. In order to ensure that the switches do not slow down the charging process, switches that drive larger capacitors will be made larger, reducing their resistance. However, this will increase the overall power dissipation of the ADC, as this is a part of the trade-off required in order to achieve the desired bandwidth.

\subsubsection{Switches for the Top Plate of the Capacitor Array}

The switch required for the top plate of the capacitor array needs to transmit either $\frac{1}{2} V_{D D}$, or remain tri-stated, or 'open-circuited'. This can be achieved with a simple analog transmission gate.

\subsubsection{Output Buffers}

After the digital output data is deemed valid, it is passed through a 10-bit register. It is then driven by large CMOS buffers to an off-chip load. These buffers need to be 
large and may introduce switching noise to the analog circuitry, resulting in a need for a separate power supply for these buffers.

Further explanations of each of these components and their implementations in this design can be found in Chapter 5 .

\subsection{ADC Circuit Architecture Summary}

The architecture used for the presented SAR ADC is based on charge redistribution in a sampling binary weighted capacitor array. The design will avoid the use of a sampleand-hold circuit in order to reduce the power requirement for the ADC. Therefore, the architecture used will be comprised of a comparator, a capacitor array, low-power CMOS digital logic for the SAR register, and analog switches. The SAR ADC has an input range from $\frac{1}{2}$ VDD to VDD and the main phases of the conversion cycle are:

(i) Analog data, $V_{i n}$, is sampled onto the bottom plate of the capacitor array, while the top plate is set to $\frac{1}{2} \mathrm{VDD}$, shown in Figure 4.1 .

(ii) Bottom plates of the capacitors in the array are forced to $\frac{1}{2} \mathrm{VDD}$, causing the top plate to become $V_{D D}-V_{i n}$, shown in Figure 4.2 .

(iii) Approximation stage: the digital SAR logic controls the switching, starting with the most significant bit (MSB), and working down to the least significant bit (LSB). The comparator determines if the voltage on top of the plate of the capacitor array is higher or lower than $\frac{1}{2}$ VDD and outputs a digital 0 or 1 respectively. Ten clock cycles are required for a 10-bit conversion, shown in Figure 4.3 and Figure 4.4.

(iv) An extra four clock cycles are required to sample the analog signal, force it negative, and output the digital data after it is found to be valid, resulting in a total 
of 14 clock cycles for approximating one 10-bit cycle.

The SAR register is implemented using registers and digital gates, and its output is the output of the ADC, after the data is found to be valid. The comparator determines whether the top plate of the capacitor array is higher or lower than analog ground, $\frac{1}{2} V_{D D}$. The binary-weighted capacitor array allows for the charge redistribution algorithm to be used in the SAR ADC architecture. The charge stored on the capacitor array acts as a sample-and-hold circuit. A variable attenuation capacitor was used in order to avoid the need for very large capacitors, potentially up to $100 \mathrm{pF}$ for a 10 -bit ADC. 


\section{Chapter 5}

\section{SAR ADC Design Components}

In order for the $\mathrm{ADC}$ to meet the requirements noted in Table 2.1, a successive approximation ADC architecture is implemented. This architecture has four main components for the presented design: the comparator, the successive approximation register, the capacitor array, and analog switches. Each component of the complete $\mathrm{ADC}$ is designed and simulated in this section. The schematics and layout are presented, with explanations for the choices that were made during the design and layout process.

\subsection{Comparator Architecture}

\subsubsection{Comparator Design}

The initial stage of the comparator is shown in Figure 5.1. It uses a differential amplifier with an NFET input stage, followed by an inverter. Analog_In is the analog input to the comparator, which will be compared to the voltage at Tune. Tune is generally set to the analog ground voltage, $\frac{1}{2} V_{D D}$, but it may be used to correct any potential input offset voltage. Transistor M5 is part of the current mirror, which is biased in the subthreshold region of operation, with a $V_{G S}<V_{t}$, which is 
approximately $0.35 \mathrm{~V}$ for the process used. In order to keep the offset voltage low, the bias current needs to stay constant. This is ideal for a circuit operating in the subthreshold region of operation, since the drain current remains almost independent of $V_{d s}$, as long as $V_{d s}>3 \frac{k T}{q}$.

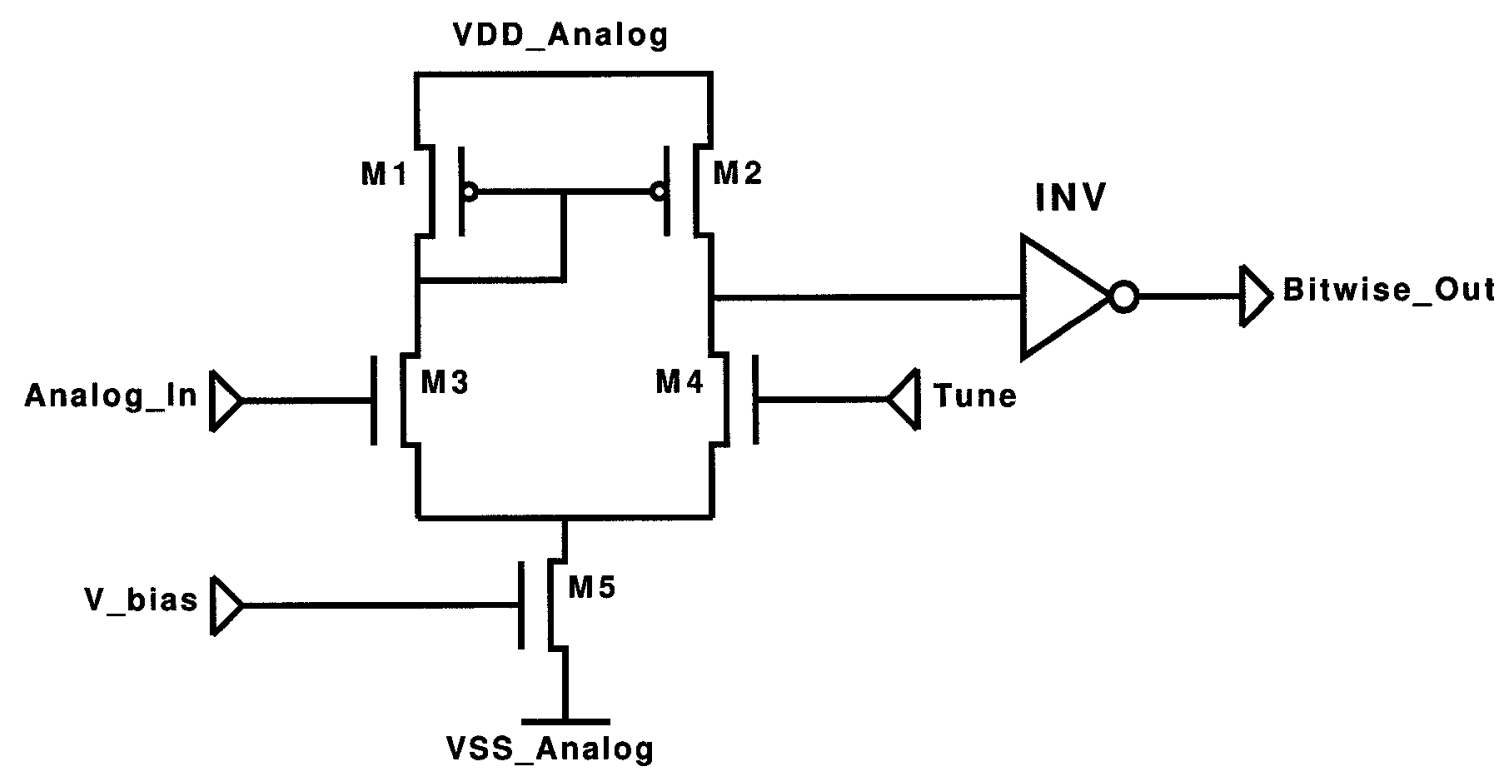

Figure 5.1: Schematic for the first stage of the comparator design.

Observing Figure 5.1, as Analog_In is increased past Tune, more current flows through the M3 transistor branch, than in the M4 branch, resulting in M2 being a stronger pull on the output than M4, driving the output to a high digital value. Similarly, when Analog_In is below Tune, more current flows through the M4 branch than the M3 branch, resulting in M4 being a stronger pull than M2, driving the output of the differential stage to a low digital value. The differential gain of the differential amplifier, as shown in [24] is

$$
A_{V}=g_{m N}\left(r_{02} \| r_{04}\right)
$$

where $r_{02}$ is the output resistance of $\mathrm{M} 2, r_{04}$ is the output resistance of M4, and $g_{m N}$ 
is the subthreshold transconductance for the NMOS transistor, M4, given by [24] as $\left(\frac{I_{D}}{n V_{T}}\right)$. The gain will also be influenced by the load placed at the output of the circuit, $C_{L}$, which modifies Equation 5.1 to

$$
A_{V}=g_{m N}\left(r_{02}\left\|r_{04}\right\| \frac{1}{s C_{L}}\right)
$$

According to Equation 5.2, the differential gain of the differential amplifier can be increased by reducing the output load capacitance.

\subsubsection{Offset Voltage for the Comparator}

In order to verify that the comparator is sufficient for a 10-bit ADC, processing variation and device mismatch need to be included in the simulation. These simulations determine the dynamic offset voltage of the comparator. This was done using Monte Carlo analysis with 200 simulation runs, with process and mismatch parameters included and no correlations assumed between the MOSFETs. Monte Carlo simulations give outputs based on statistical probabilities of certain process and mismatch errors occurring, using previously collected statistical data. The results of the simulation are shown in Figure 5.2. Ideally, the offset needs to be less than the least significant bit (LSB). For a $0.6 \mathrm{~V}$ supply voltage, and a 10-bit architecture, the LSB is $\frac{0.6 \mathrm{~V}}{2^{10}}=586 \mu \mathrm{V}$. The offset voltage needs to be less than this value in order to be confident in the comparator design. According to Figure 5.2, the average offset voltage due to processing and device mismatch variation is $-149 \mu \mathrm{V}$, while the standard deviation of the distribution is $3.2 \mathrm{mV}$. This results in a yield of only $14.5 \%$, or 29 of the 200 simulations meet the LSB requirement. Since the sample size of 200 runs using Monte Carlo simulations gave a yield of only $14.5 \%$, there is no need to increase 
the sample size for the simulation performed in this section. An attempt was made to reduce the offset voltage of the comparator in order to ensure a greater isolation from process and device mismatch variation.

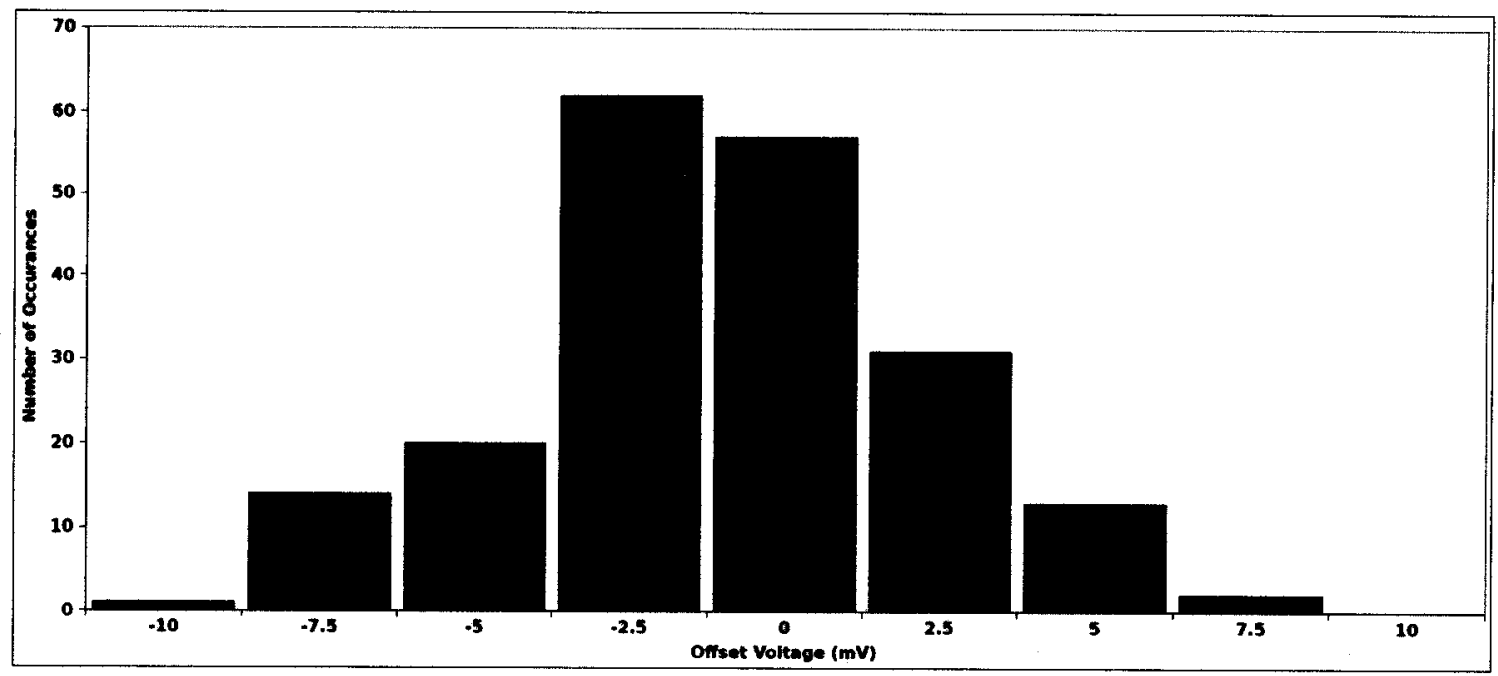

Figure 5.2: Offset voltage for the presented comparator.

\subsubsection{Offset Voltage Correction Circuit}

Section 5.1.2 shows that process variation and device mismatch can severely degrade the accuracy of the presented comparator. This offset voltage can be corrected using external digital signal processing, in order to save power [13]. However, since the intent of this design is to reduce the power for the complete sensor circuit, an analog offset voltage correction circuit will be implemented in the design. The offset voltage correction was implemented using an architecture with two stages, as shown in Figure 5.3, where the comparator from Figure 5.1 will be used as a pre-amplifier. In [31], it is shown that a way to correct the offset is to store the offset voltage on a capacitor during the reset phase, followed by canceling it during the comparison stage. A similar differential amplifier with NFET input transistors that was used in the first stage is 


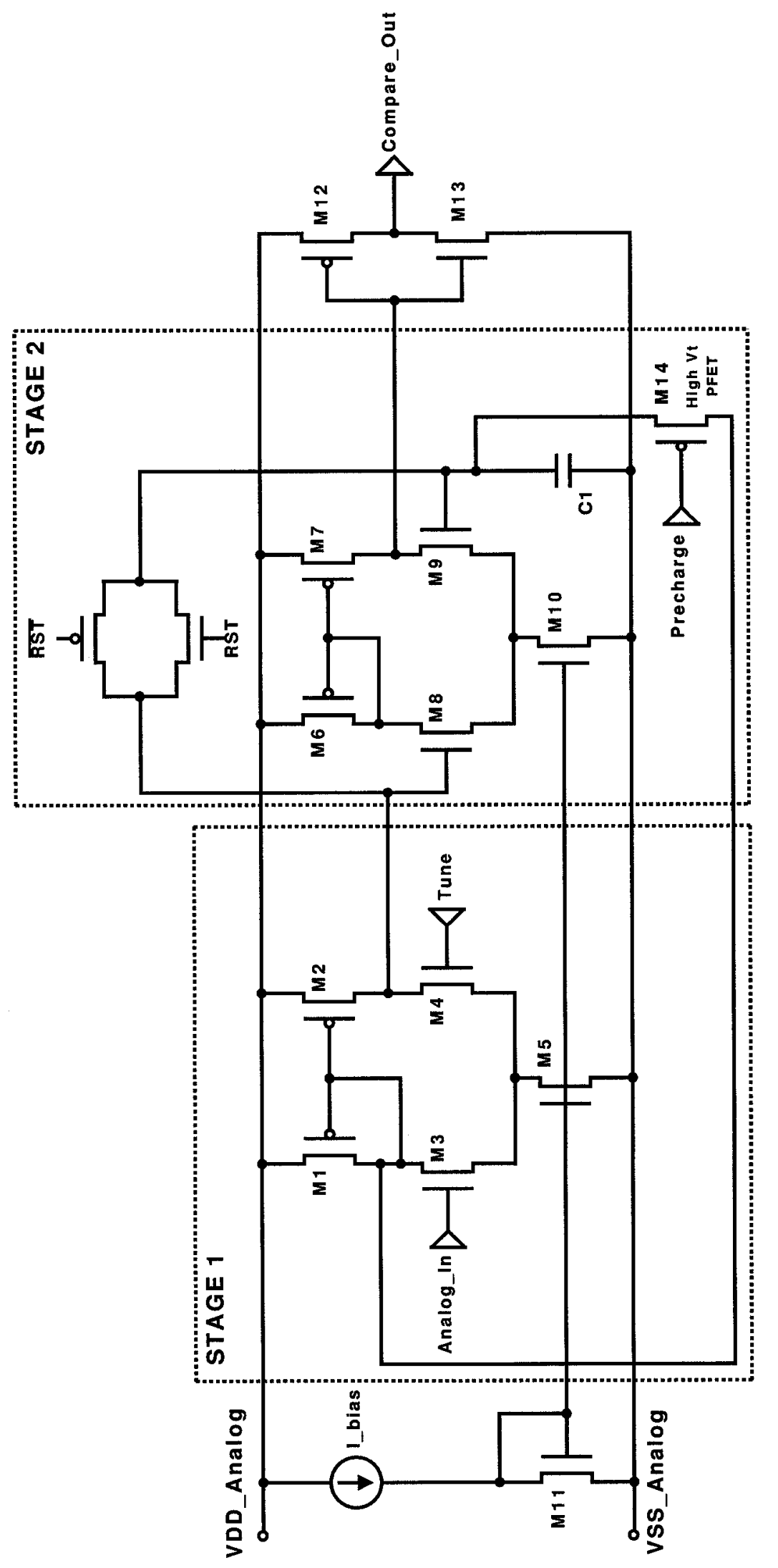

苞

苞

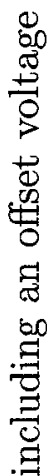

8

疍

웅

3

.

ธิ่

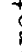

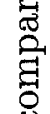

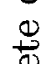

롱

일

4

苞

نे

垔 
used for the second stage, as shown in the circuit of the full comparator in Figure 5.3, while the sizes of the devices are shown in Table 5.1.

During the sampling phase of the ADC, the Analog_In and Tune signals are both set to analog ground, which is $\frac{1}{2} V_{D D}$. This forces the output from the first stage to be at some voltage level near $\frac{1}{2} V_{D D}$ plus to any offset voltage. The output from the first stage, including the offset voltage is stored on capacitor $C 1$ from Figure 5.3.

During conversion, the second stage acts as the comparator, with one input connected to the stored value of the offset voltage, from $C 1$. The second input is connected to the output of the first stage of the comparator. Since the offset voltage is present on both inputs, the differential amplifier will remove the offset that was present after the first stage. Although some offset is expected at the second stage of the comparator, this will be less significant, since the desired signal has already been amplified.

Transistor M12 is present in order to eliminate the need to precharge the capacitor to GND or VDD before storing the offset voltage, which would cause a significant reduction in speed. The capacitor would, otherwise, need to charge or discharge to the desired value, which is at approximately $\frac{1}{2} V_{D D}$. Instead, the capacitor is precharged to the value connecting the gates of the PFET transistors M1 and M2. This value is equal to the output of the first stage when no offset voltage affects the circuit, meaning that during the sampling phase, the capacitor only needs to charge or discharge by the offset voltage. This will take a small fraction of the time when compared to charging or discharging $\frac{1}{2} V_{D D}$. The precharge for the capacitor is the first step and it occurs when the Precharge signal from Figure 5.3 is low, as shown in Figure 5.4. 
A precharge needs to occur at the beginning of any conversion cycle, or at the end of the previous conversion cycle. The signal going LOW allows the PMOS device M14 to act as a short to the top of the capacitor, C1. Since both inputs to the differential amplifier in stage 1 are $\frac{1}{2} V_{D D}$, then the output from the amplifier is expected to be approximately $\frac{1}{2} V_{D D}$, which is the value charged on the capacitor. The next step is to trigger the $R S T$ signal, as shown in Figure 5.4, resulting in the transmission gate shown in Fig 5.3 allowing current to pass through. While the $R S T$ is triggering, the comparator samples the analog input, which is set to $\frac{1}{2} V_{D D}$, and the capacitor is charged with the offset voltage from the output of stage 1 . Once the $R S T$ returns to LOW, the conversion cycle begins.

Table 5.1: Sizes for the devices used for the presented comparator

\begin{tabular}{|c|c|c|c|}
\hline Device & \multicolumn{2}{|c|}{ Size } & Value \\
\hline \hline Transistor & Width & Length & Type \\
\hline M1 & $12 \mu m(6 \times 2 \mu m)$ & $2 \mu m$ & PFET \\
\hline M2 & $12 \mu m(6 \times 2 \mu m)$ & $2 \mu m$ & PFET \\
\hline M3 & $10 \mu m(4 \times 2.5 \mu m)$ & $400 \mathrm{~nm}$ & NFET \\
\hline M4 & $10 \mu m(4 \times 2.5 \mu m)$ & $400 \mathrm{~nm}$ & NFET \\
\hline M5 & $4 \mu m(4 \times 1 \mu m)$ & $1 \mu m$ & NFET \\
\hline M6 & $3 \mu m(3 \times 1 \mu m)$ & $2 \mu m$ & PFET \\
\hline M7 & $3 \mu m(3 \times 1 \mu m)$ & $2 \mu m$ & PFET \\
\hline M8 & $5 \mu m(4 \times 1.25 \mu m)$ & $500 n m$ & NFET \\
\hline M9 & $5 \mu m(4 \times 1.25 \mu m)$ & $500 n m$ & NFET \\
\hline M10 & $2 \mu m(2 \times 1 \mu m)$ & $1 \mu m$ & NFET \\
\hline M11 & $2 \mu m(2 \times 1 \mu m)$ & $1 \mu m$ & NFET \\
\hline M12 & $1 \mu m$ & $120 n m$ & PFET \\
\hline M13 & $500 n m$ & $120 n m$ & NFET \\
\hline M14 & $360 n m$ & $240 n m$ & High Vt PFET \\
\hline \hline Capacitor & Width & Length & Value \\
\hline C1 & $10 \mu m$ & $15 \mu m$ & $315.35 f F$ \\
\hline
\end{tabular}



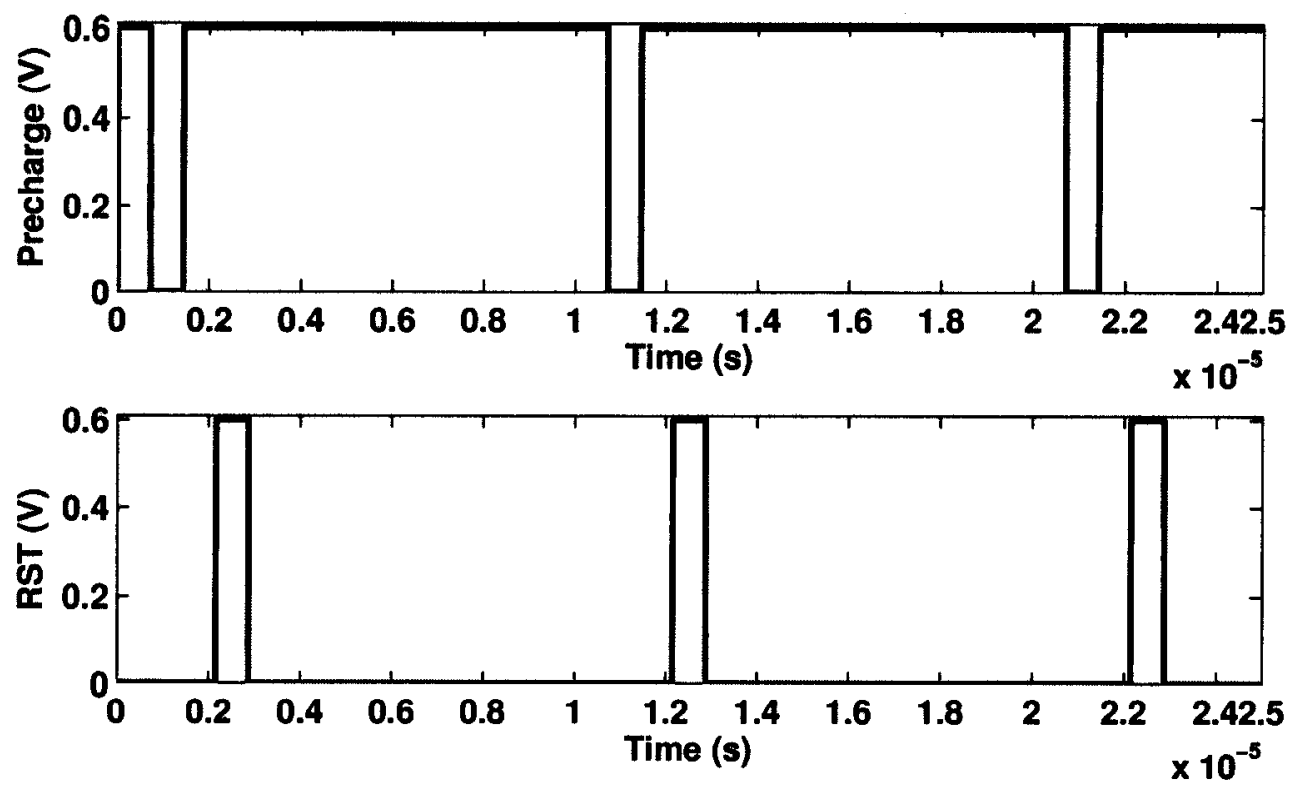

Figure 5.4: Timing of the Precharge and RST signals for the offset voltage correction circuit.

\subsubsection{Offset Voltage for the Two-Stage Comparator}

Processing variation and device mismatch was included in simulations of the full twostage comparator from Figure 5.3 in order to find the dynamic offset voltage. This was done using Monte Carlo analysis with 200 simulation runs, similar to the simulations performed for the first stage of the comparator. The Monte Carlo simulation was used with process and mismatch parameters included and no MOSFET correlations. The results of the simulation are shown in Figure 5.5. According to Figure 5.5, the average offset voltage due to processing and device mismatch variation is $-118.95 \mu \mathrm{V}$, while the standard deviation of the distribution is $515.5 \mu \mathrm{V}$. This is a significant improvement, of approximately six times, compared to the standard deviation observed by the comparator without the offset voltage correction circuitry, as now the yield has increased to $73.2 \%$. Comparing Figure 5.5 with Figure 5.2, it is visually evident 
that the offset voltage correction circuit has succeeded in improving the comparator's tolerance of process variation and device mismatch. Had the Monte Carlo simulations given a yield closer to $100 \%$, a larger number of simulation runs would be required in order to have a more precise view of the statistical probability of the comparator having an offset voltage that does not fit the requirement.

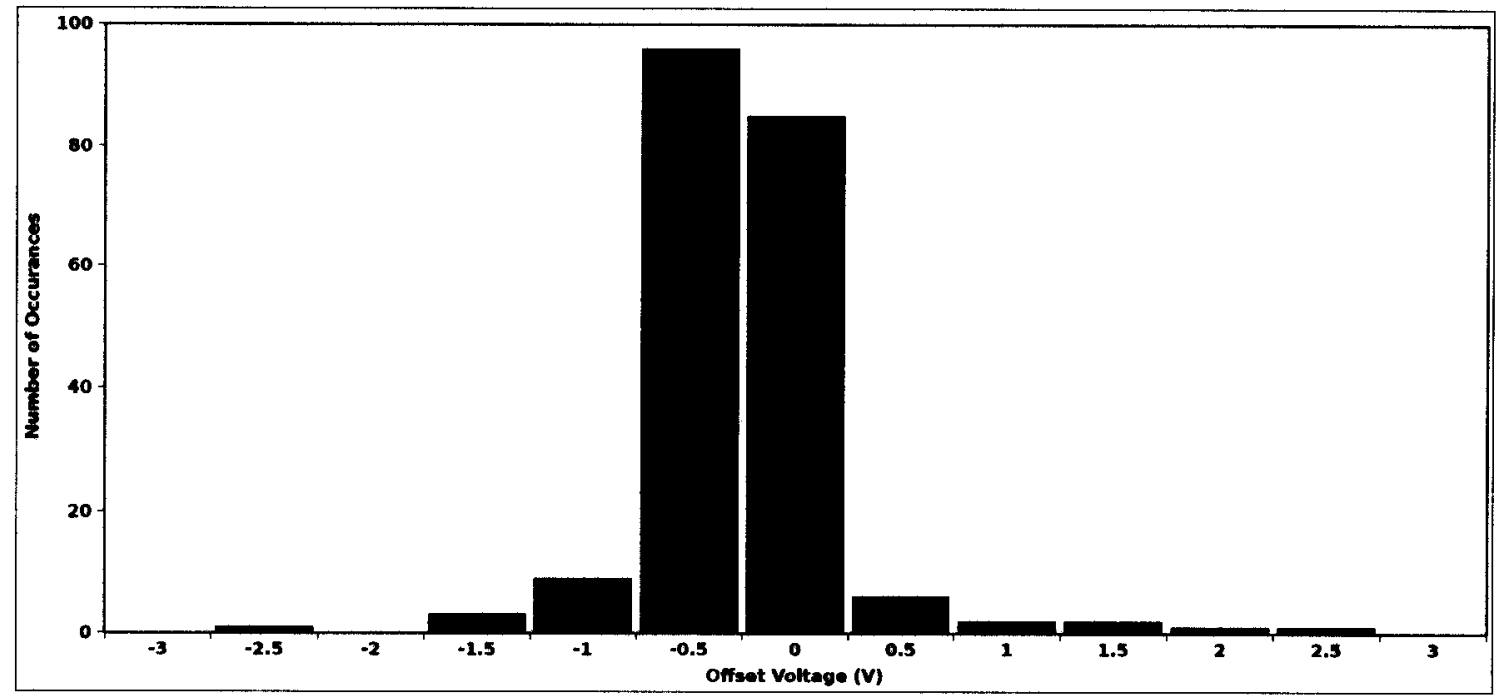

Figure 5.5: Offset voltage of the presented comparator after implementing the offset correction circuit.

In order to verify that the presented comparator improves the input referred offset voltage, one of the inputs, Tune was swept from $-10 \mathrm{mV}$ to $+10 \mathrm{mV}$ away from its desired value of $\frac{1}{2} V_{D D}[24]$. The results are shown in Figure 5.6, where it is evident that after the offset voltage circuit is implemented, there is a much smaller variation in the output offset voltage. With no offset correction, the output offset voltage varies from $-6.7 \mathrm{mV}$ to $14 \mathrm{mV}$ as the Tune voltage is varied from $-10 \mathrm{mV}$ to $10 \mathrm{mV}$, respectively. However, after implementing the offset correction circuit, the output offset voltage varies from $-1.34 \mathrm{mV}$ to $1.79 \mathrm{mV}$ as the Tune voltage is varied from $-10 \mathrm{mV}$ to $10 \mathrm{mV}$, respectively. 


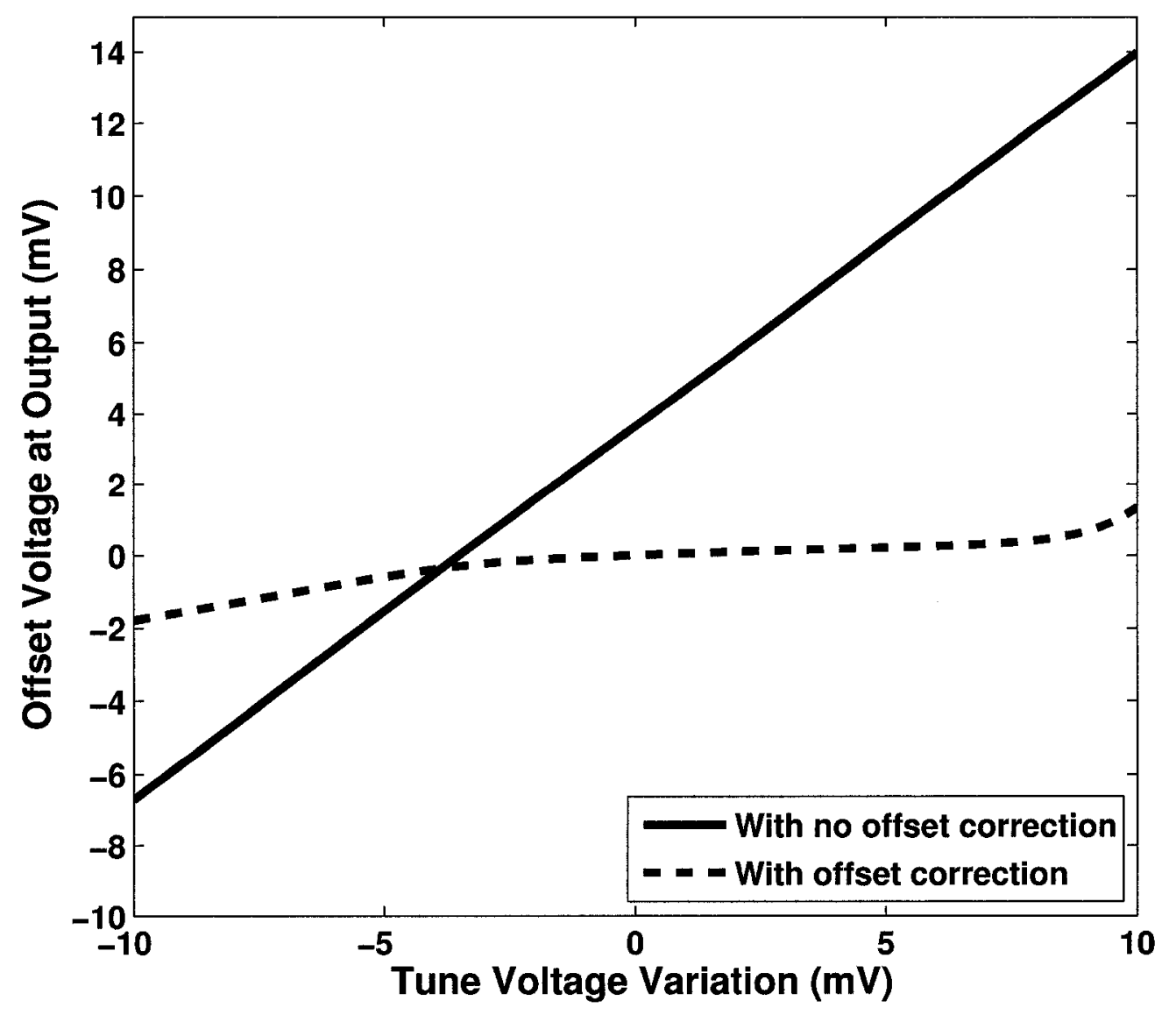

Figure 5.6: Offset voltage of the comparator shown before and after the offset voltage correction circuit is implemented as a result of a varying Tune voltage. 


\subsubsection{Comparator Simulations \\ Bias Current, Power, and Bandwidth Simulation}

The current for the comparator is set using a current mirror, as shown in Figure 5.3 with M11, M5, and M10. The bias voltage is generated using a diode-connected transistor, M11, which has a $\mathrm{W} / \mathrm{L}$ ratio of $2 \mu \mathrm{m} / 1 \mu \mathrm{m}$. Table 5.2 shows the required bias current in order to achieve an offset voltage that is smaller than the least significant bit (LSB) voltage of $\frac{V_{D D}}{2^{10}}$. It also shows the achievable bandwidth at that bias current, as well as the power dissipated when an input at the stated frequency is passed through the comparator. It is evident that for each voltage simulated, from $0.3 \mathrm{~V}$ to $1.0 \mathrm{~V}$, the current mirror is biased in the subthreshold region, as the gate bias voltage varies from $90 \mathrm{mV}$ to $216 \mathrm{mV}$. Both values are well below the threshold voltage for the MOSFET technology used, which is approximately $350 \mathrm{mV}$. The lowest power dissipation is noted at the lowest source voltage, $0.3 \mathrm{~V}$. The comparator can achieve a frequency of $85 \mathrm{kHz}$, resulting in a potential bandwidth of $6 \mathrm{kS} / \mathrm{s}$, while dissipating approximately $20 \mathrm{nW}$. The highest power dissipation also corresponds to the highest bandwidth and highest source voltage. With a $V_{D D}$ of $1.0 \mathrm{~V}$, the comparator achieved a frequency of approximately $5 \mathrm{MHz}$, corresponding to a bandwidth of $357 \mathrm{kS} / \mathrm{s}$, while dissipating $6.25 \mu \mathrm{W}$. The bottleneck for the achievable speed of the comparator is the charging and discharging speed of the capacitor through a transmission gate, and not the comparator itself.

The delay through a transmission gate, with a capacitive load of $C_{\text {load }}$ is stated 
Table 5.2: Simulated bias current, power, and bandwidth of the comparator.

\begin{tabular}{|c|c|r|l|l|r|}
\hline$V_{D D}(\mathrm{~V})$ & LSB $(\mu V)$ & $I_{\text {bias }}(n A)$ & $V_{\text {bias }}(m V)$ & Power $(\mathrm{nW})$ & Bandwidth $(\mathrm{kHz})$ \\
\hline \hline 0.3 & 293 & 21 & 90.5 & 18.78 & 85 \\
\hline 0.4 & 391 & 29 & 90.7 & 36.87 & 150 \\
\hline 0.5 & 488 & 48 & 114.2 & 77.55 & 200 \\
\hline 0.6 & 586 & 83 & 130.5 & 164.28 & 300 \\
\hline 0.7 & 683 & 155 & 169.7 & 382.06 & 650 \\
\hline 0.8 & 781 & 296 & 170.5 & 1510 & 1500 \\
\hline 0.9 & 879 & 571 & 192.8 & 3310 & 3200 \\
\hline 1.0 & 976 & 1074 & 216.0 & 6250 & 5000 \\
\hline
\end{tabular}

in [24] as $t_{P H L}=t_{P L H}=0.7 \cdot\left(R_{n} \| R_{p}\right) \cdot$, resulting in a bandwidth of

$$
\text { Bandwidth }=\frac{1}{0.7 \cdot\left(R_{n} \| R_{p}\right) \cdot C_{l o a d}}
$$

where $R_{n}$ is the ON resistance of the NFET, $R_{p}$ is the ON resistance of the PFET, and $C_{\text {load }}$ is the capacitive load.

\section{Temperature Simulation}

The comparator was simulated for temperature variation to ensure that it can perform while implanted inside the human body. Table 5.3 shows the results of these simulations and characterizes the performance of the comparator under temperature variation. The goal was to measure the temperature variation that is allowed in order to satisfy an offset voltage less than $\frac{1}{2} L S B$. At higher source voltages the temperature variation does not seem to be an issue; however, when the comparator is in deep subthreshold, there will be some offset voltage variation when the ADC is exposed to extremes in temperature. When operating at $0.3 \mathrm{~V}$, the comparator has a temperature range from $-5^{\circ} \mathrm{C}$ to $56^{\circ} \mathrm{C}$, which is sufficient for implantable biosensors, since the body temperature of any human patient will never extend beyond that range. With supplies above $0.6 \mathrm{~V}$, the comparator can function within $\frac{1}{2} \mathrm{LSB}$ for temperatures below 
Table 5.3: Variation in comparator offset voltage due to temperature variation.

\begin{tabular}{|c|c|c|c|}
\hline Source Voltage, $V_{D D}$ & LSB & $\frac{1}{2}$ LSB & Temperature Range $\left({ }^{\circ} \mathrm{C}\right)$ \\
\hline \hline $0.3 \mathrm{~V}$ & $293 \mu V$ & $146.5 \mu V$ & $-5^{\circ} \mathrm{C}$ to $56^{\circ} \mathrm{C}$ \\
\hline $0.4 \mathrm{~V}$ & $391 \mu V$ & $195.5 \mu V$ & $-15^{\circ} \mathrm{C}$ to $70^{\circ} \mathrm{C}$ \\
\hline $0.5 \mathrm{~V}$ & $488 \mu V$ & $244.0 \mu V$ & $-136^{\circ} \mathrm{C}$ to $115^{\circ} \mathrm{C}$ \\
\hline $0.6 \mathrm{~V}$ & $586 \mu V$ & $293.0 \mu V$ & $<136^{\circ} \mathrm{C}$ \\
\hline $0.7 \mathrm{~V}$ & $683 \mu V$ & $341.5 \mu V$ & $<160^{\circ} \mathrm{C}$ \\
\hline
\end{tabular}

$-136^{\circ} \mathrm{C}$

\subsubsection{Comparator Layout}

The layout of the complete comparator circuit from Figure 5.3 is shown in Figure 5.7. The dimensions of the layout are $71 \mu m$ by $47 \mu m$. Layout for each MOSFET pair is performed using interdigitated layouts in order to improve matching between FETs. The transistors are placed in a guard ring in order to avoid noise injection from the digital components of the chip, as suggested in [31].

\subsection{Successive Approximation Register Design}

The successive approximation register schematic is shown in Figure 5.8, which is based on [38] and is used in other low-power SAR ADC designs in [35] and [13]. The digital library that was designed is composed of standard CMOS gates, including NAND gates, NOR gates, and inverters with various drive strengths. The devices were designed by optimizing sizes to reduce the required power, without much care for the speed, since the digital circuit is not expected to be the speed bottleneck for the designed ADC. The NMOS and PMOS transistors were also sized to equate drive strength, resulting in the PMOS devices having a width that is approximately twice 


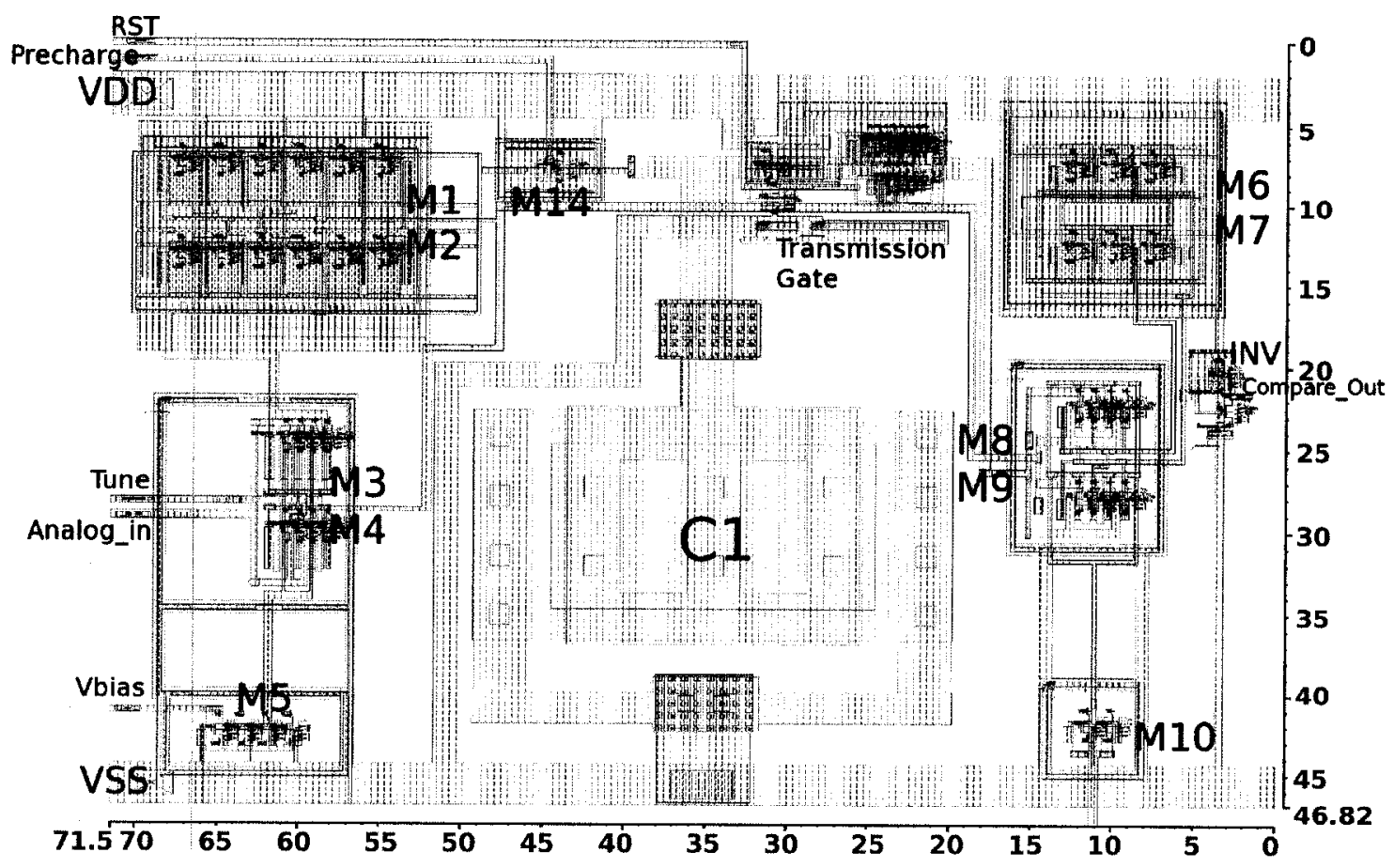

Figure 5.7: Layout of the comparator circuit.

that of the NMOS transistors. This ensures that the noise margins are maximized and that the switching occurs at approximately $\frac{1}{2} V_{D D}$. The sizes of the transistors were also minimized in order to minimize current dissipation. The essential component of this SAR is a D-flip-flop with an active-LOW reset and an active-LOW set, an output and its inverse. The flip-flop design from [18] was used, which uses six 3-input NAND gates, as shown in Figure 5.10.

The input and output signals required for the SAR are shown in Table 5.4.

\subsubsection{SAR Operation}

The circuit that implements the successive approximation algorithm and the control circuitry is shown in Figure 5.8. The algorithm works as a shift register, where the first flip-flop is triggered to HIGH by either a reset signal, or by the previous 


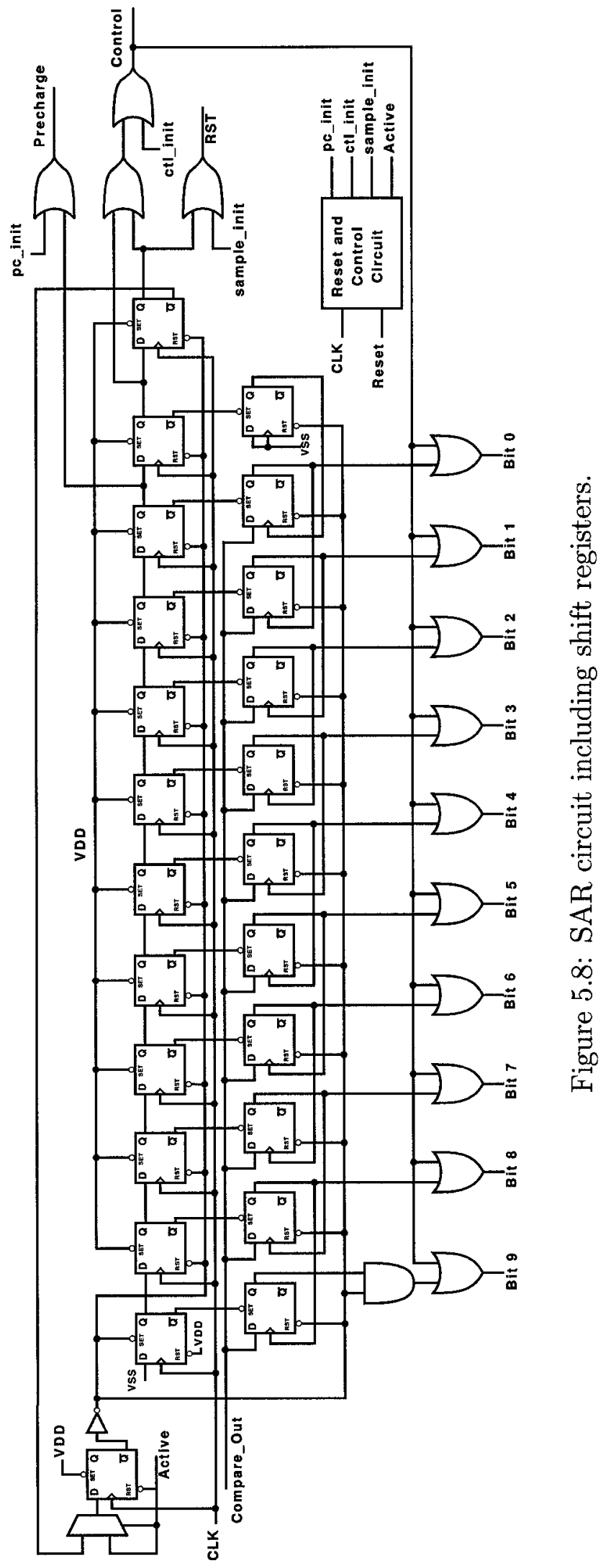




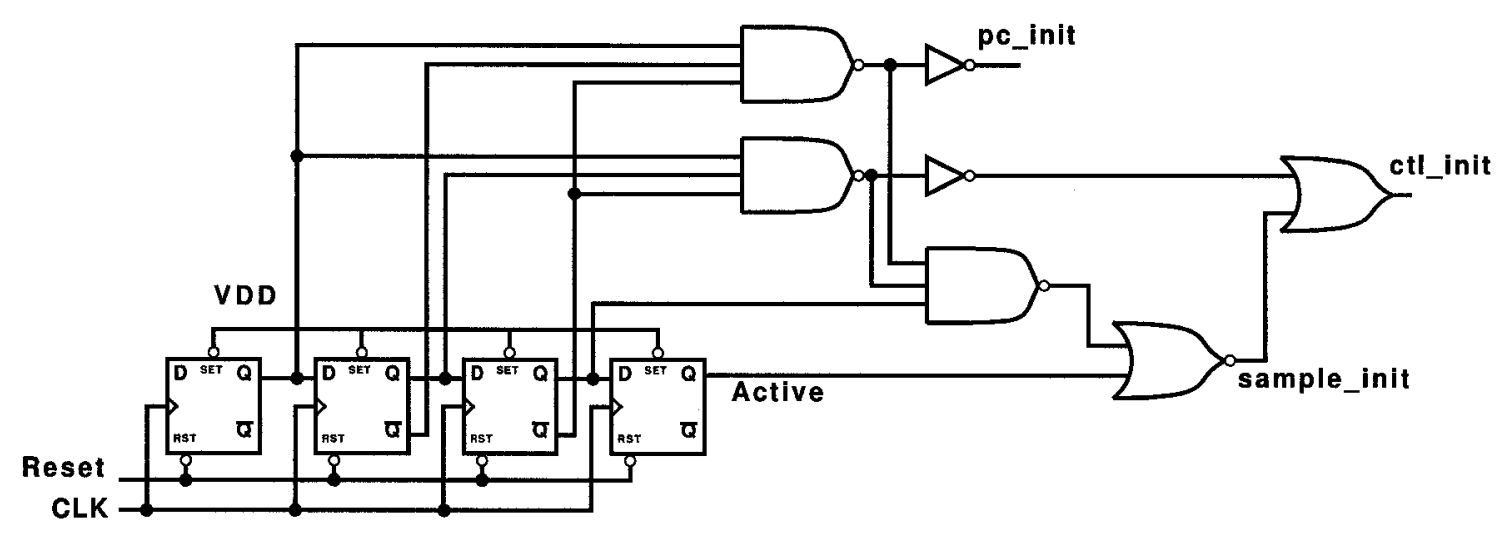

Figure 5.9: Reset and control circuit from Figure 5.8.

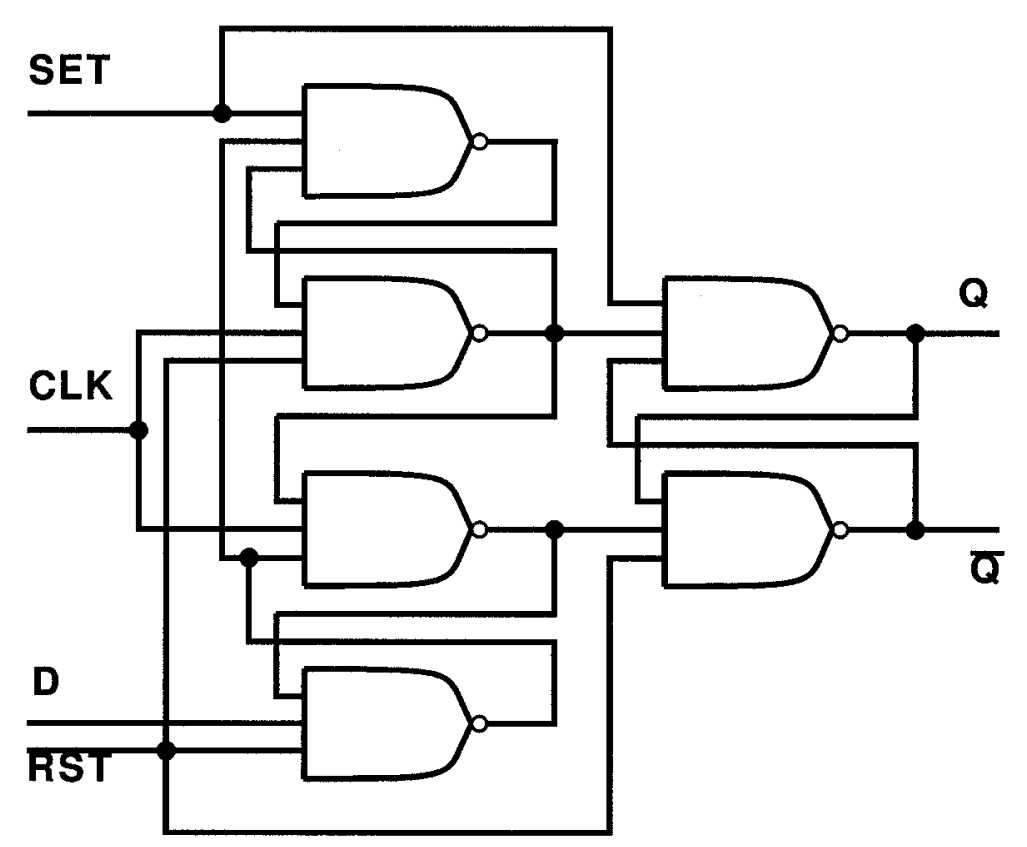

Figure 5.10: D-flip-flop design used in the successive approximation register from Figure 5.8. 
Table 5.4: Explanation for the input and output signals of the successive approximation register from Figure 5.8.

\begin{tabular}{|l|l|l|}
\hline Signal Name & Signal Type & Function \\
\hline \hline VDD & Digital Power & Supply voltage for the digital SAR \\
\hline VSS & Digital Power & Ground voltage for the digital SAR \\
\hline CLK & Clock Input & $\begin{array}{l}\text { Clock to be used by digital circuit } \\
(14 \times \text { Bandwidth })\end{array}$ \\
\hline Compare_Out & Input & $\begin{array}{l}\text { Output of the comparator, passed to the } \\
\text { SAR }\end{array}$ \\
\hline Reset & Input & $\begin{array}{l}\text { Asynchronous active-LOW reset for the dig- } \\
\text { ital circuit }\end{array}$ \\
\hline Precharge & Output & $\begin{array}{l}\text { Activate pre-charge for comparator, reset top } \\
\text { plate of the capacitor array }\end{array}$ \\
\hline Control & Output & $\begin{array}{l}\text { Used to set desired voltage on the bottom of } \\
\text { the capacitor array during sampling }\end{array}$ \\
\hline RST & Output & $\begin{array}{l}\text { Reset the comparator including the offset } \\
\text { correction capacitor }\end{array}$ \\
\hline Bit 9 to Bit 0 & 10-bit Output & Bits 9 to 0 for the ADC \\
\hline
\end{tabular}

conversion cycle's completion, when the rest of the flip-fops are set to LOW. The digital 1 generated in the first flip flop propagates through the rest of the registers at one per clock cycle. Each top flip-flip turns on the lower flip flops by triggering the $S E T$ input, causing the Compare_Out signal from the comparator to be read at the input. If this input is HIGH, the output of the flip flop will then clock the previous flip-flop and allow it to store the Compare_Out value. The signals from the bottom flip-flops either remain at logic 0 or are changed to logic 1 depending on the value of the output of the comparator. After all bits have been assessed, a new cycle begins, which is set by the Reset and Control Circuit. The reset and control circuitry generates all the necessary signals to initiate a new conversion cycle, including the signals required to precharge the capacitor in the offset voltage correction circuitry from the comparator. 


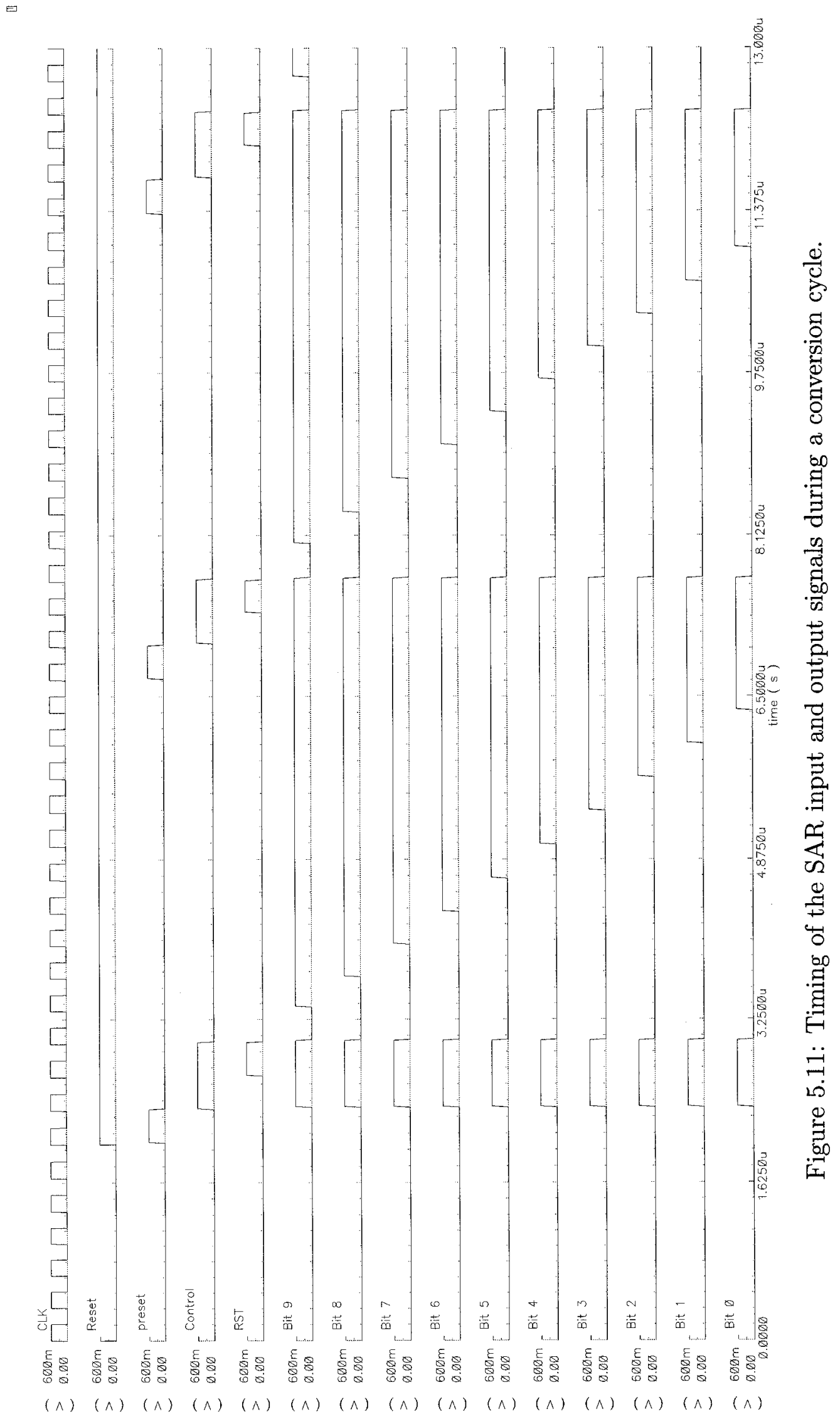

Reproduced with permission of the copyright owner. Further reproduction prohibited without permission. 
Figure 5.11 shows the timing of the input and output signals of the successive approximation register during a conversion cycle. After the reset is set to high, the first signal that is triggered is Precharge. This signal precharges the offset voltage correction capacitor in the comparator, as discussed in Section 5.1.1. The next signal that is triggered is the Control signal, which opens the transmission gates to set the

top of the capacitor array to the analog ground voltage, $\frac{1}{2} V_{D D}$, while sampling the analog input signal to the bottom of the capacitor array. The RST signal is triggered after, causing the the comparator to perform offset correction while still sampling the analog input, since Control is still triggered. When Control goes back to LOW, the bottom of the capacitor arrays is switched to analog ground, causing the top plate of the capacitor to be $V_{D D}-V_{i n}$. ADC is now set to perform the successive approximation algorithm, which takes 10 clock cycles, 1 clock cycle per bit. The total time it takes to perform one 10-bit conversion is 14 clock cycles. The layout of the full successive approximation register and the control circuitry for the ADC is shown in Figure 5.12. The dimensions of the layout are $205 \mu m$ by $150 \mu m$.

\subsubsection{SAR Power Dissipation}

To simulate the power dissipation of the successive approximation register, along with the control circuitry, a frequency of $1.4 \mathrm{MHz}$ was used, corresponding to a $100 \mathrm{kHz}$ input signal. Figure 5.13 shows the relationship between the supply voltage, $V_{D D}$ and the power dissipated by the SAR digital circuit. As the voltage is increased, the power appears to increase at a quadratic rate. Figure 5.14 shows the relationship between the input frequency and the SAR power. As expected, it is clear that power dissipation 


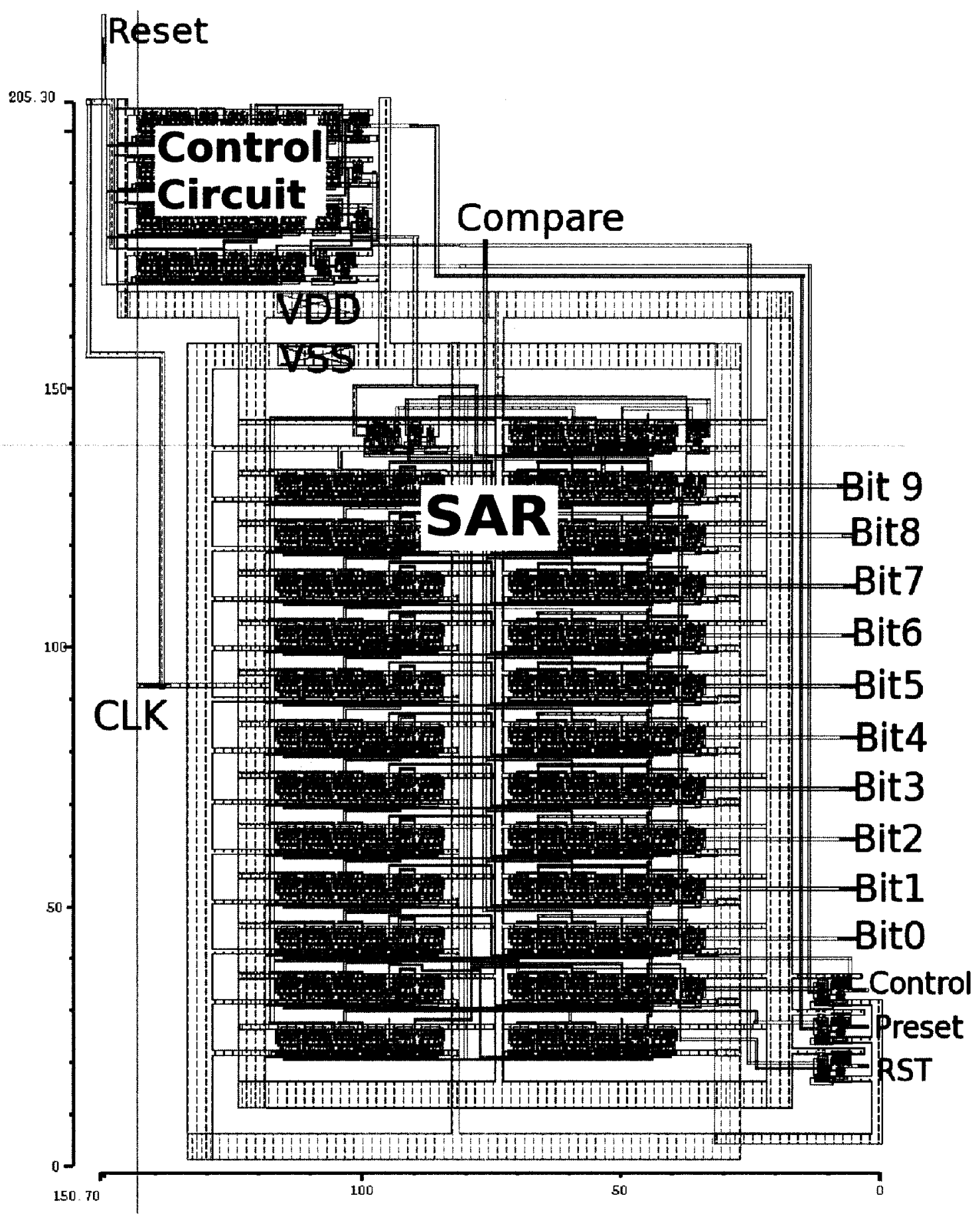

Figure 5.12: Complete layout of the SAR circuit along with the digital circuit for the control circuitry from Figure 5.8. 
increases with an increasing frequency, and this occurs at a seemingly linear rate. The explanation for the quadratic increase in power due to a $V_{D D}$ increase and the linear increase in power due to a frequency increase is found in the equation for power of a digital inverter, from [39]:

$$
P=\frac{1}{2} V_{D D}^{2} C_{L} f+I_{l e a k} V_{D D}
$$

where $I_{\text {leak }}$ is the leakage subthreshold current that is present when $V_{G S}$ is approximately $0 \mathrm{~V}$ and gate leakage, $C_{L}$ is the load capacitance, including the output capacitance of the digital gate, and $f$ is the frequency of operation. Equation 5.4 shows that power should increase quadratically with $V_{D D}$, as in Figure 5.13, and that power should increase linearly with the frequency, $f$, as in Figure 5.14.

When process variation and device mismatch are included in the SAR simulation, power measurements vary according to Figure 5.15. This figure shows power variation when the SAR circuit is simulated with 100 runs of Monte Carlo simulations at a $V_{D D}$ of $0.6 \mathrm{~V}$, using a $1.4 \mathrm{MHz}$ clock, which corresponds to a bandwidth of $100 \mathrm{kHz}$. The average power is found to be $117 \mathrm{nW}$ with a standard deviation of $8.2 \mathrm{nW}$. The Monte Carlo simulation performed in this section is based on statistical probability of process and mismatch variation with no pre-determined correlations between any MOSFETs.

\subsection{Binary Weighted Capacitor Array Design}

The binary weighted capacitor array was implemented with MIMCAPs from the process library, as in Figure 5.18. The capacitor array has one side shorted while the other side of each individual capacitor is connected to an analog switch. The switch sets the 


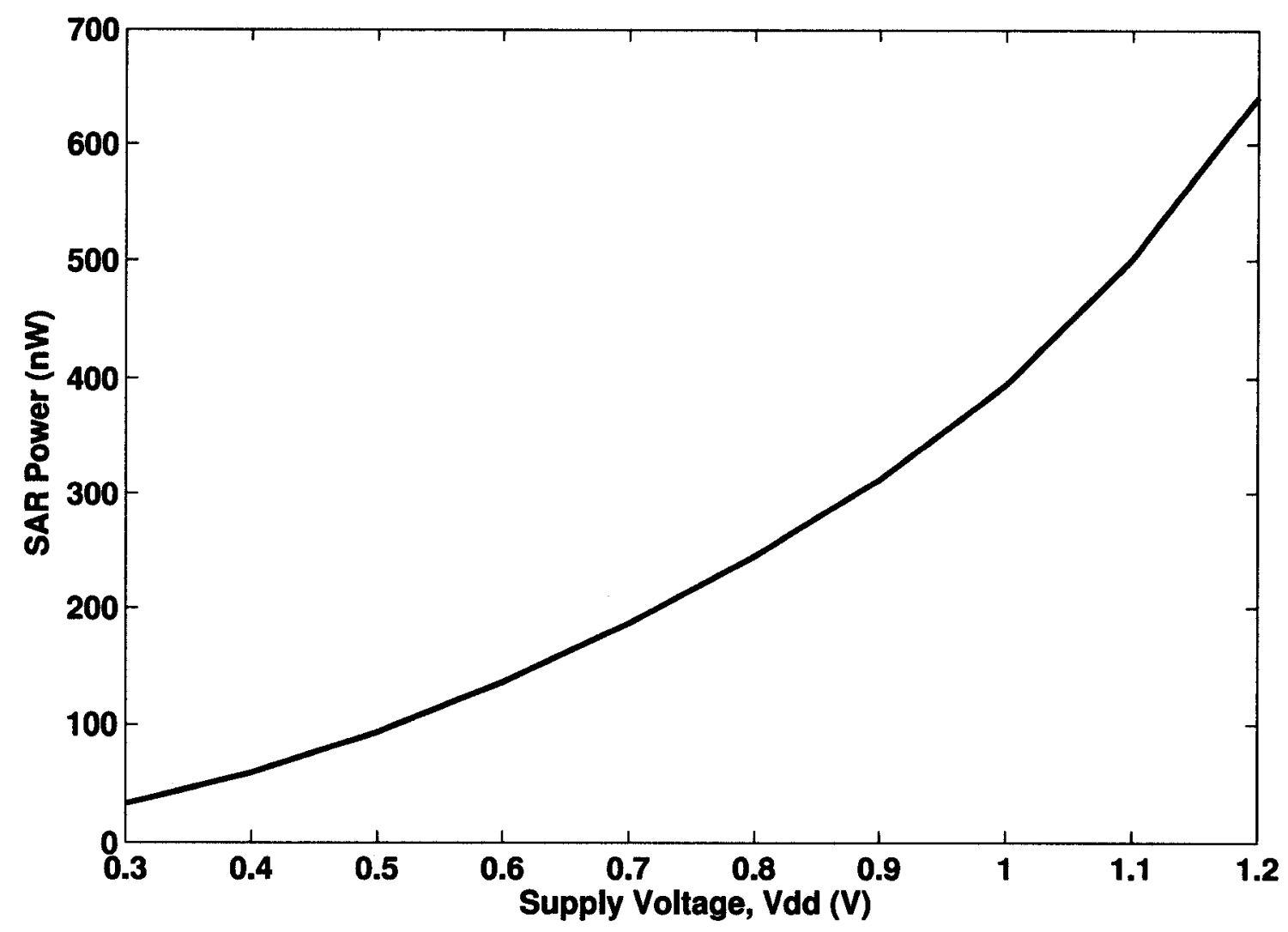

Figure 5.13: Total digital power dissipation of the SAR with varying supply voltages at a frequency of $1.4 \mathrm{MHz}$, corresponding to a sample rate of $100 \mathrm{kS} / \mathrm{s}$. 


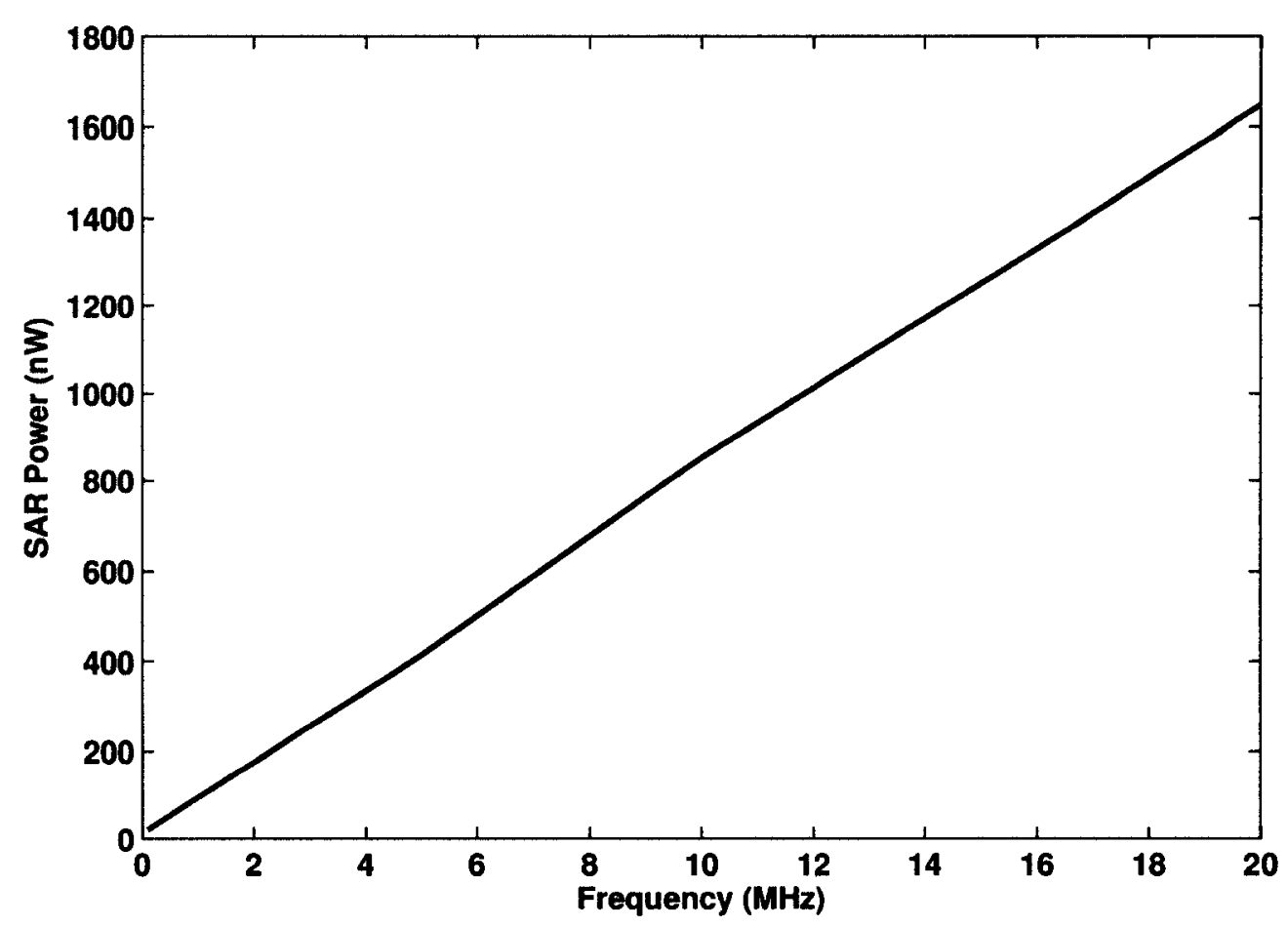

Figure 5.14: Total digital power dissipation of the SAR with varying input frequency at a $0.6 \mathrm{~V}$ power supply. 


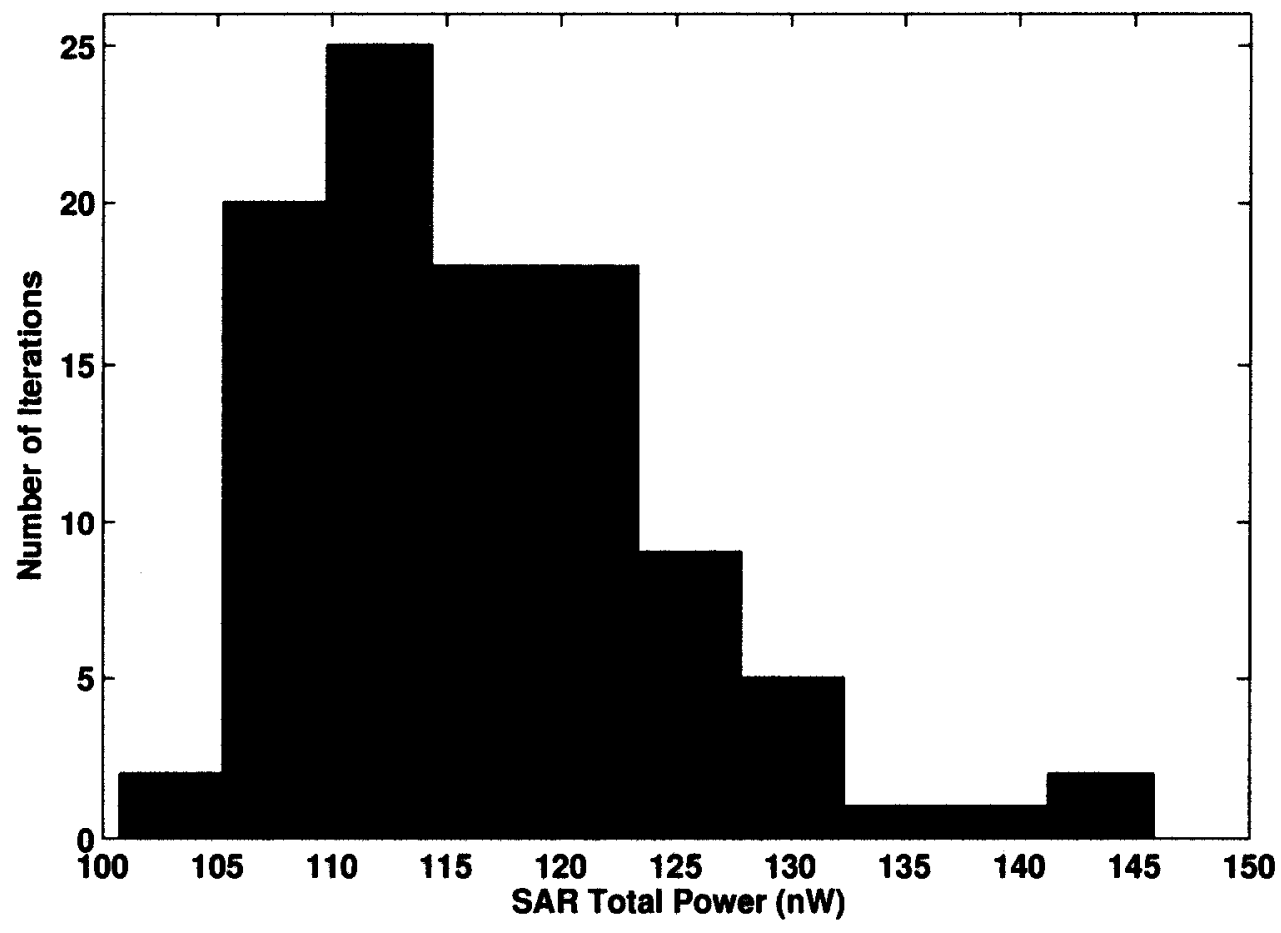

Figure 5.15: Monte Carlo statistical analysis simulation of the SAR digital power. Simulation performed for 100 runs with process variation and mismatch, a $V_{D D}$ at $0.6 \mathrm{~V}$, and a clock at $1.4 \mathrm{MHz}$.

\begin{tabular}{|c|c|c|c|c|c|c|c|c|c|}
\hline $512 C$ & $256 \mathrm{C}$ & $128 \mathrm{C}$ & $64 C$ & $32 \mathrm{C}$ & $16 \mathrm{C}$ & $8 C$ & $4 C$ & $2 C$ & c \\
\hline
\end{tabular}

Figure 5.16: Binary weighted capacitor array schematic for a 10-bit ADC. 
voltage at the bottom of the capacitor to either $V_{\text {ref }}\left(V_{d d}\right)$ or $\operatorname{Analog}_{-} G N D\left(\frac{V_{d d}}{2}\right)$, depending on the conversion stage of the bit in question. The effective capacitance of this switch has an effect on the operation of the capacitor array. The switch capacitance is effectively added onto each individual capacitor in the array, resulting in potential nonlinearities and dynamic range limitations of the ADC. For example, when the most significant bit (MSB) is being evaluated, the largest voltage stored on all capacitors is divided between $512 \mathrm{C}$ and the sum of the remaining capacitors in the array, which is ideally $512 \mathrm{C}$. However, the MSB capacitor is $512 \mathrm{C}+C_{\text {switch }}$, while the sum of the remaining capacitors is $512 \mathrm{C}+9 C_{\text {switch }}$. If the switch capacitance is larger than the smallest capacitor in the array, then the ADC will lose one bit of resolution. Therefore, the smallest capacitor in the array, C, needs to be large enough so that the error caused by the switch capacitance does not influence the conversion by more than 1 LSB.

The size of the capacitors in the capacitor array directly influences the speed of the ADC. However, making the capacitors small can result in errors during conversion due to the previously-mentioned effective switch capacitance, as well as due to capacitor thermal noise [24]. The thermal noise of a capacitor is shown in [40] as

$$
v_{n}=\sqrt{\frac{k_{B} T}{C}}
$$

where $k_{B}$ is the Boltzmann constant and $\mathrm{T}$ is the temperature in Kelvin. A plot of the function from Equation 5.5, at room temperature, results in the graph shown in Figure 5.17 . When the supply voltage is $0.3 \mathrm{~V}$, the least significant bit is $293 \mu \mathrm{V}$. The smallest capacitor in the array needs to be greater than $48 \mathrm{fF}$ in order for the noise 
to be less than $1 \mathrm{LSB}$. A noise of $97 \mu \mathrm{V}$ is noted when a capacitor of $440 \mathrm{fF}$ is used and this value is chosen as the unit capacitor. Increasing the unit capacitance further will increase the time needed to charge and discharge the capacitor array, resulting in slowed ADC performance.

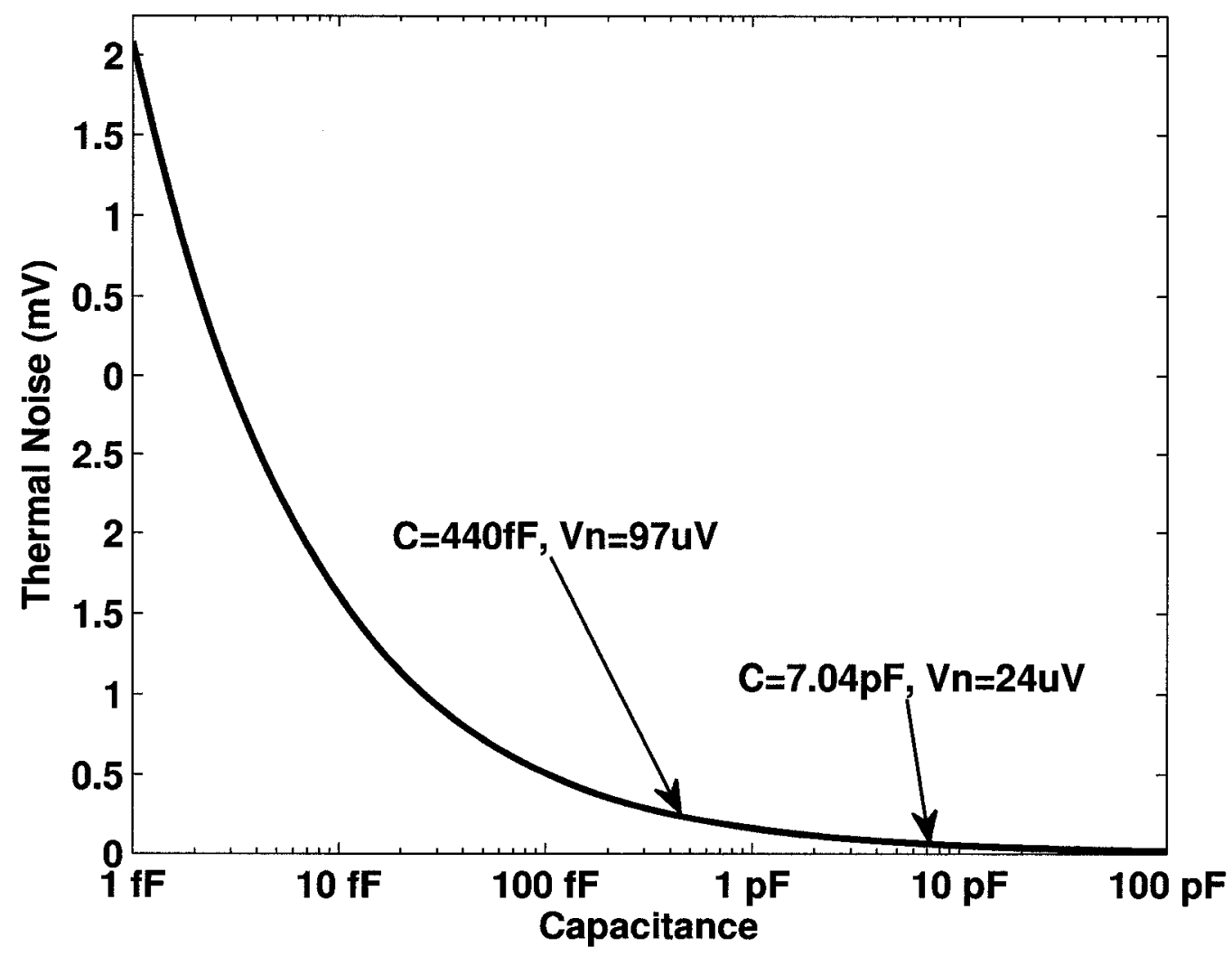

Figure 5.17: Thermal noise of a capacitor at room temperature $(\mathrm{T}=300 \mathrm{~K})$.

Since the array shown in Figure 5.16 contains large capacitors, the area required by these capacitors would likely result in not meeting the area requirement set out in Table 2.1. In addition to an increased area, the time to fully charge the large capacitors would also be significant, reducing the speed of the designed ADC. This can be solved using a series attenuation capacitor $[24,41]$. The capacitor is placed in series with the capacitor array split in two, each with its own top plate switch. 
This helps reduce the chip area significantly and allows for faster speeds, since the largest capacitor that requires charging is $16 \mathrm{C}$ as opposed to $512 \mathrm{C}$. The size of the attenuation capacitor, from [24] is:

$$
C_{A T T}=\frac{\Sigma L S B_{-} \text {Capacitors }}{\Sigma M S B_{-} \text {Capacitors }} C
$$

where the sum of the LSB capacitors is $32 \mathrm{C}$, the sum of the MSB capacitors is $31 \mathrm{C}$, and the size of the unit capacitor, $\mathrm{C}$, is $440 \mathrm{fF}$. Therefore, the MSB capacitors add up to $14.08 \mathrm{pF}$ and the LSB capacitors add up to $13.64 \mathrm{pF}$. The resulting attenuation capacitor, CATT, should be $454 \mathrm{fF}$. After extracting the layout to include parasitic capacitances in the circuit, it was found that the attenuation capacitor needs to be $647 \mathrm{fF}$ in order to achieve optimal operation.

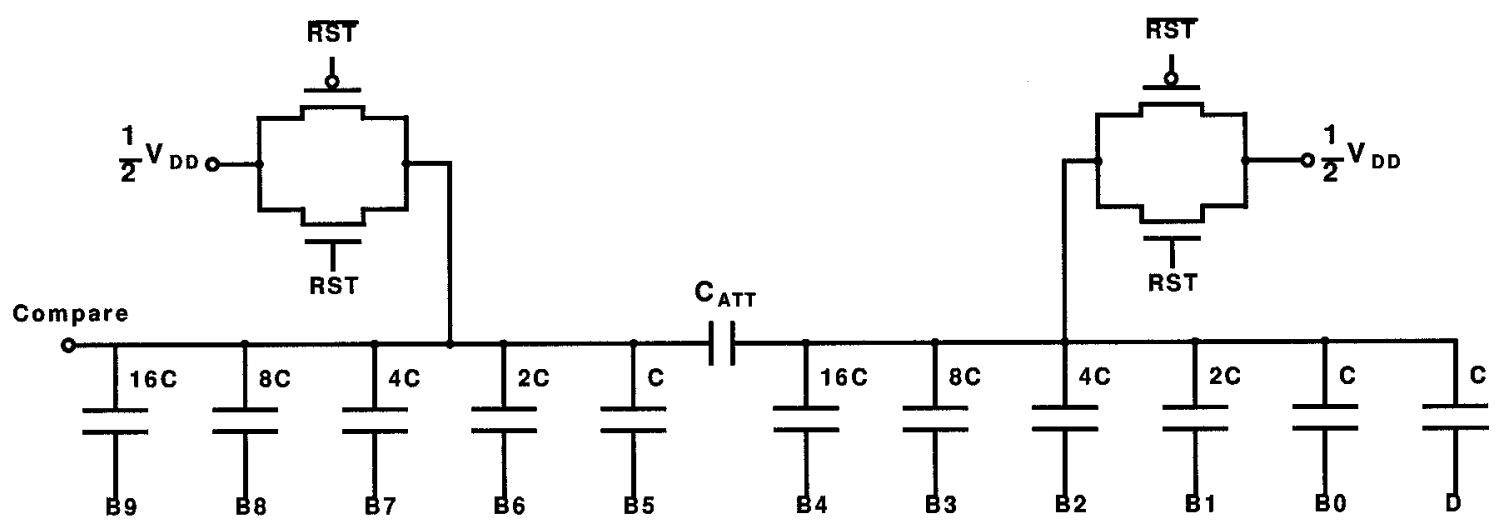

Figure 5.18: Binary weighted capacitor array circuit including analog switches.

The attenuation capacitor, $C_{A T T}$ from Figure 5.18, is susceptible to process variation and, after manufacture, it is likely that the value of this capacitance will be different from ideal. The capacitor arrangement from Figure 5.18 is very sensitive to any parasitic capacitances because $C_{A T T}$ is a floating capacitor; it is not directly 
charged or discharged by any voltage source. A truly floating capacitor is not available in standard bulk CMOS technologies [42], such as the $0.13 \mu m$ technology used in the presented design. In specialized processes, such as silicon-on-sapphire, used in [17] and [42], a floating capacitor is available and can be designed to be very accurate. In order to design a single series attenuation capacitor in a standard bulk CMOS technology, substrate parasitics need to be taken into account. The designs in [18] showed that with a single attenuation capacitor and no tuning implementation, the ADC lost approximately 0.5 effective bits during dynamic operation when compared to the tuned attenuation capacitor. The use of the attenuation capacitor can also degrade INL and DNL of the ADC if the capacitor is not the ideal value [17]. Implementing a tuned attenuation capacitor is advantageous because it allows for the topology of the ADC design to be easily transferable to another technology, such as the $90 \mathrm{~nm}$ or $65 \mathrm{~nm}$ CMOS processes. The capacitors in the array are all scalar multiples of each other and can be laid out with a common centroid arrangement to ensure all capacitors experience the same etching and doping conditions. The attenuation capacitor is not a scalar multiple of the unit capacitor, $\mathrm{C}$, and matching it to the rest of the capacitor array can be very challenging. An easier alternative is to make the attenuation capacitor tunable [18].

\subsubsection{Tunable Attenuation Capacitor Design}

A way to mitigate the effects of the parasitic capacitance on the value of the series attenuation capacitor is to make the capacitor tunable, as shown in Figure 5.19. The digitally tunable capacitor schematic, shown in Figure 5.19, allows for 5-bit tuning 
of the series attenuation capacitor. This allows us to vary the capacitor value until an appropriate value is found to offset the mismatch due to process variation. The equation that governs the capacitance is

$$
\begin{aligned}
C_{A T T}= & \left(\frac{2}{C}+\frac{C A P_{1}}{C}\right)^{-1}+\left(\frac{2}{C}+\frac{C A P_{2}}{2 C}\right)^{-1}+\left(\frac{2}{C}+\frac{C A P_{3}}{3 C}\right)^{-1} \\
& +\left(\frac{2}{C}+\frac{C A P_{4}}{4 C}\right)^{-1}+\left(\frac{2}{C}+\frac{C A P_{5}}{5 C}\right)^{-1}
\end{aligned}
$$

where $\mathrm{C}$ is the value of the individual capacitors and $C A P_{1}$ to $C A P_{5}$ are binary values of 1 or 0 depending if the switch is open or closed, respectively. The minimum capacitance occurs when all the switches are closed, or when the CAPx bits from Equation 5.7 are all 1, resulting in an attenuation capacitance of $C_{A T T}=2.06 C$. The maximum capacitance occurs when the switches are open, or when the CAPx bits from Equation 5.7 are all 0, resulting in an attenuation capacitance of $C_{A T T}=$ 2.5C. The circuit was optimized to perform with the capacitor select bits set to 10000 for $C A P_{1}, C A P_{2}, C A P_{3}, C A P_{4}$, and $C A P_{5}$ respectively, which results in an attenuation capacitance of 2.33C. Therefore, in order to achieve the desired series attenuation capacitor of $454 \mathrm{fF}$, each individual capacitor, $\mathrm{C}$, needs to be $\frac{454 \mathrm{fF}}{2.33}=$ $195 f F$. Since the switches add to the overall attenuation capacitance, this value is tuned using extracted simulations. After simulating the extracted ADC with parasitic capacitances, it was found that the attenuation capacitor needs to be $647 \mathrm{fF}$, and each unit capacitor, $\mathrm{C}$, needs to be $278 \mathrm{fF}$ for optimal operation. It is found that the attenuation capacitor varies between $572 \mathrm{fF}$ and $694 \mathrm{fF}$ by changing the tunable capacitors switch control inputs. During device fabrication, it is expected that the values of the capacitors will change due to process variation; however, with 5 bits of 
freedom, there are 32 different capacitor values that can be used as the attenuation capacitor in order to narrow it down to a value that produces the best performance.

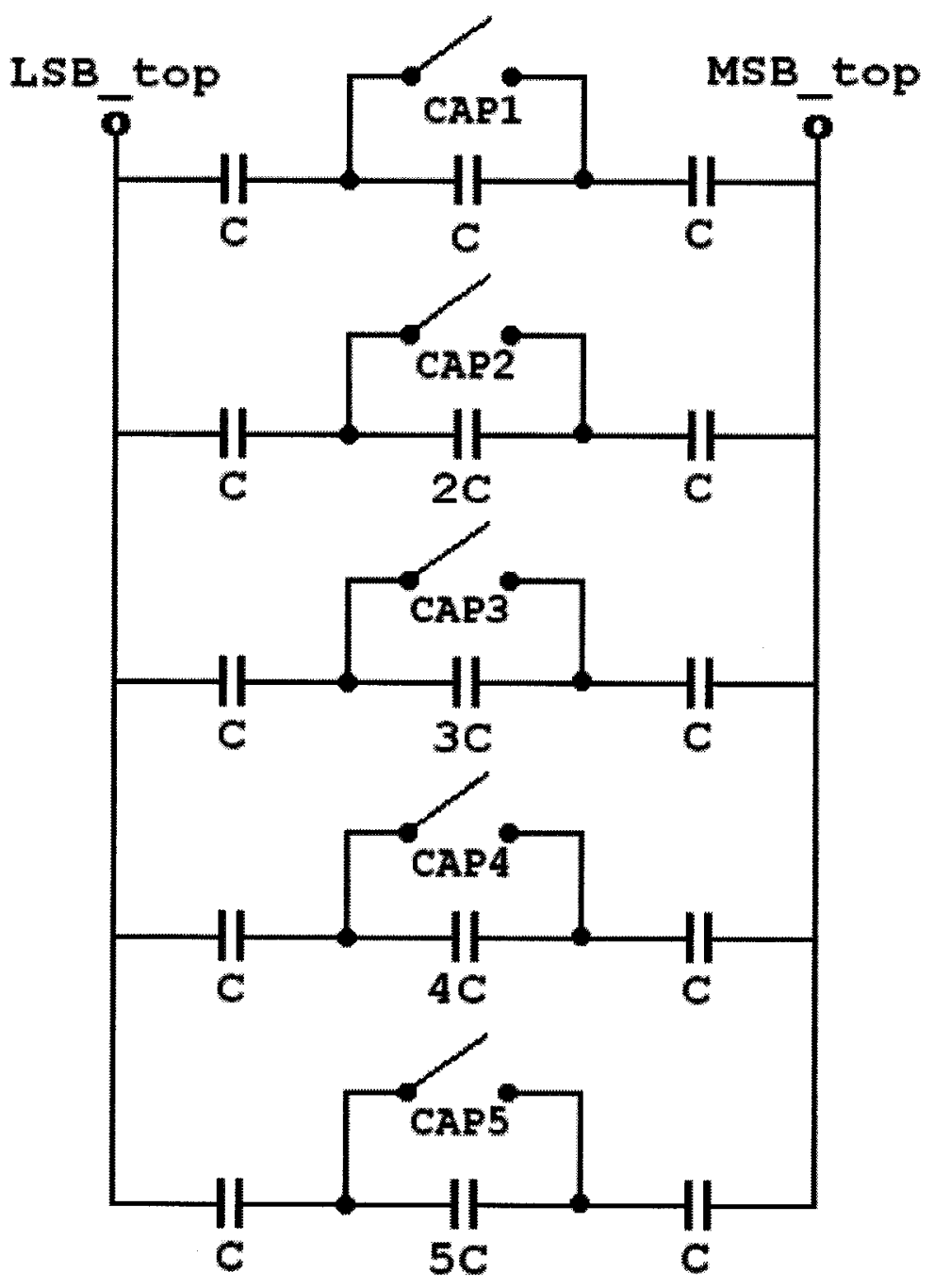

Figure 5.19: Schematic of the digitally tunable attenuation capacitor, $C_{A T T}$.

\subsubsection{Binary Weighted Capacitor Array Layout}

Any mismatch in the capacitor array can result in poor linearity of the ADC [43]. Therefore, matching of the capacitors is important in order to preserve INL and DNL of the ADC. Maximum DNL and INL errors due to capacitor mismatch are given in [24] are in Equation 5.8 and 5.9, respectively. The equations show the relative 


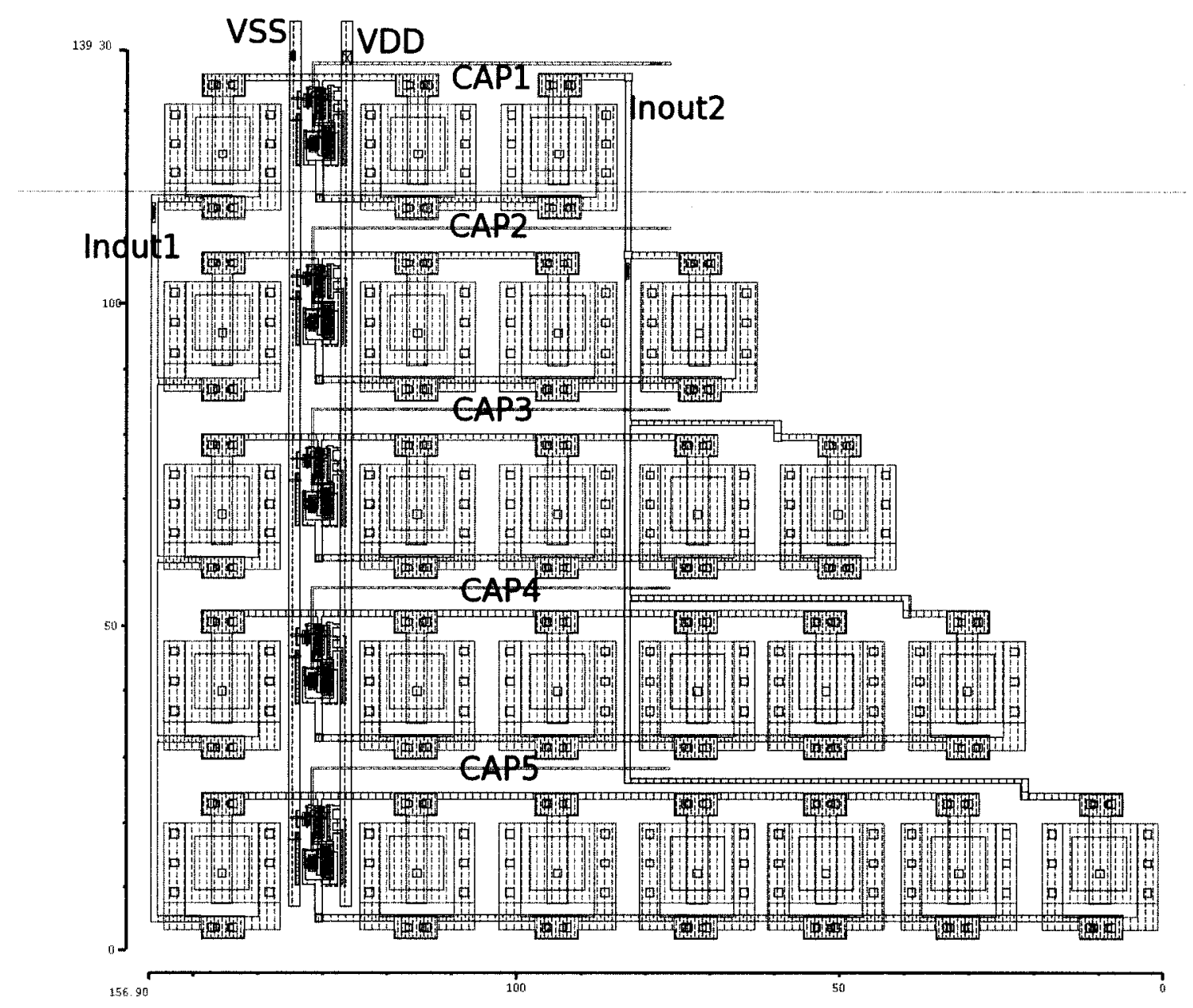

Figure 5.20: Layout of the tunable attenuation capacitor at an area of $255 \mu \mathrm{m}$ by $140 \mu m)$. 
variation in INL and DNL in terms of capacitor variation, $\Delta C$.

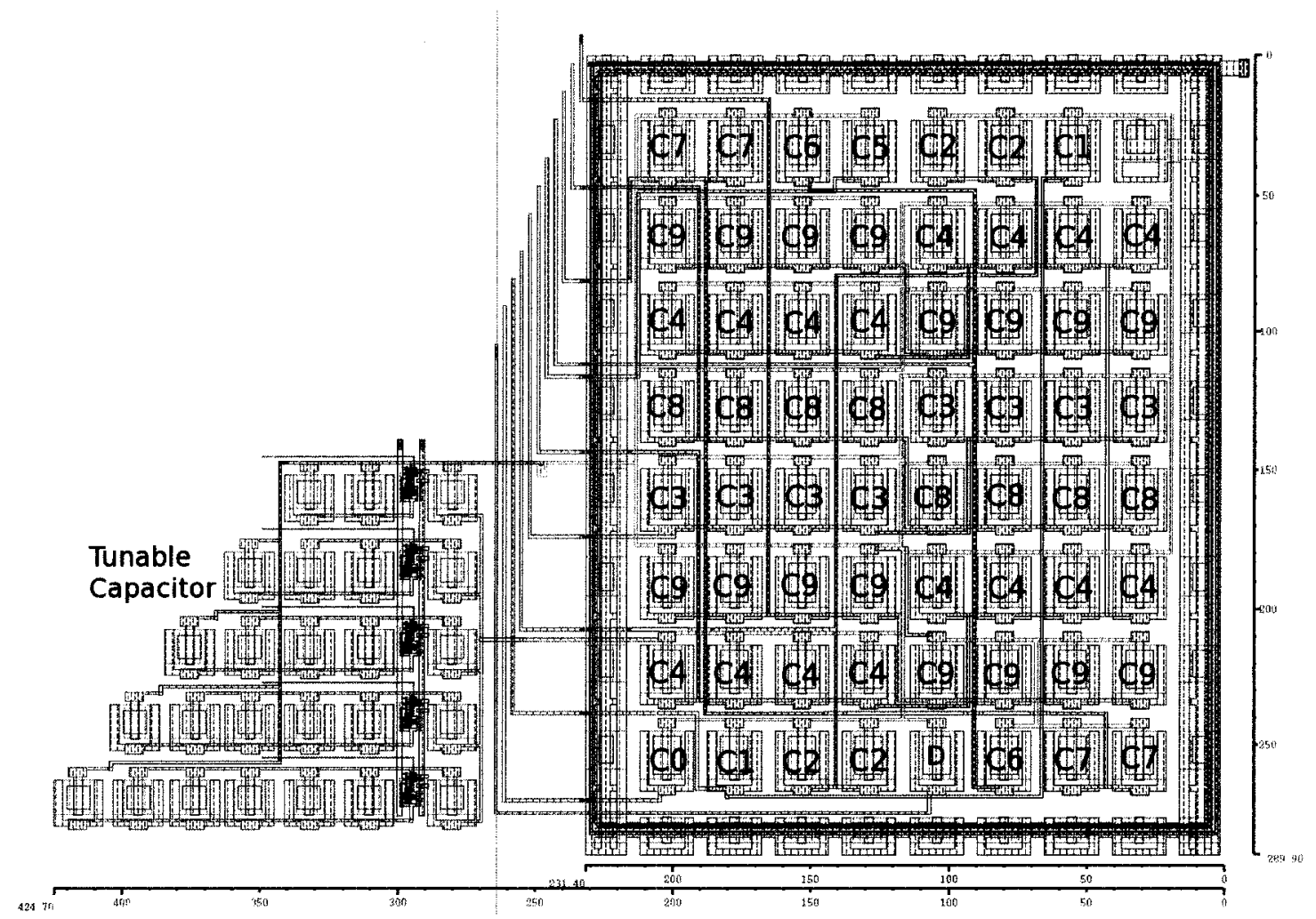

Figure 5.21: Layout of the capacitor array with dimensions of $290 \mu \mathrm{m}$ by $230 \mu \mathrm{m}$, together with the tunable attenuation capacitor (Total area of $290 \mu \mathrm{m}$ by $425 \mu \mathrm{m}$ ).

$$
\begin{gathered}
D N L_{\max }=\left(2^{N}-1\right) \cdot|\Delta C|_{\max , D N L}, \\
I N L_{\max }=\left(2^{N-1}\right) \cdot|\Delta C|_{\max , I N L}
\end{gathered}
$$

where $\Delta C$ is the maximum capacitance mismatch and $N$ is the number of bits. A common centroid layout was used in order to reduce mismatch in the capacitors due to cross-chip gradients, as is shown in Figure 5.21. There is a total of 63 unit capacitors required, which was laid out in an array of $8 \times 8$ capacitors, each designed using a MIMCAP with $15 \mu \mathrm{m} \times 14 \mu \mathrm{m}$ dimensions. In order to avoid different processing and 
etching conditions for the capacitors on the outside of the array, a ring of dummy capacitors was used around the entire array. In addition, guard rings were used to surround the capacitor array in order to improve isolation from switching components such as digital logic.

\subsection{Analog Switches and Output Buffers}

\subsubsection{Design of the Analog Switches for the SAR ADC}

There are three switches that are required for the designed SAR ADC and all switches are designed using the shortest length of transistor, $0.12 \mu \mathrm{m}$. One type of switch is required to switch between $V_{i n}$ and $V_{\text {ref }}$ as the voltage at the bottom of the capacitor array, the Sample Switch. Another switch is used to select between the output from the Sample Switch and $\frac{1}{2} V_{D D}$ for each individual bit. The third switch is used to either store $\frac{1}{2} V_{D D}$ to the top plate of the capacitor array, or to disconnect it from any input. The switch for the top plate of the capacitor is essentially a CMOS transmission gate, which is used to reset the top plate of the capacitor array before each conversion cycle. The signal that will be passed through the gate will always be $\frac{1}{2} V_{D D}$, therefore it is assured that the signal will always be between $V_{D D}$ and $V_{S S}$. The switch is sized with a $\frac{\text { PMOSwidth }}{\text { NMOSwidth }}$ ratio of $\frac{8 \mu m}{4 \mu m}$. A summary of the required switches for the capacitor array is shown in Figure 5.22.

Switch 2 from Figure 5.22 is used to switch the bottom of each capacitor that is associated with a specific bit between $\frac{1}{2} V_{D D}$ and the result from Switch 1. Most of these switches are implemented with CMOS transmission gates that are sized with a $\frac{P M O S w i d t h}{\text { NMOSwidth }}$ ratio of $\frac{2 \mu m}{1 \mu m}$; however, the switches that drive the two largest sized 


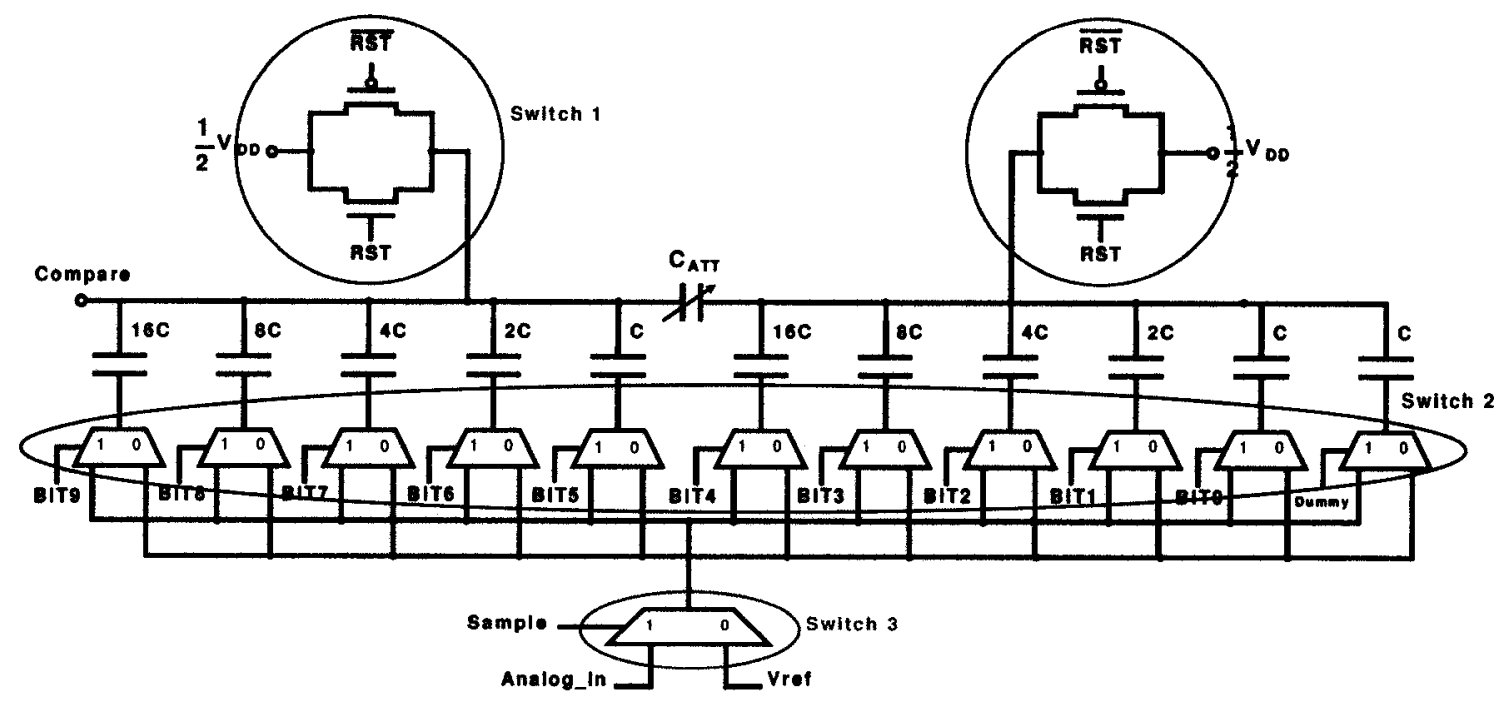

Figure 5.22: Schematic showing the switches in the ADC design.

capacitors are sized with a ratio of $\frac{8 \mu m}{4 \mu m}$. This is done for bit 3 , bit 4 , bit 8 , and bit 9 . Having a larger width-to-length ratio of a transistor allows for more current to flow through it, resulting in an increased speed for charging the larger capacitors.

The largest switch used is Switch 1 from Figure 5.22, which is used to drive the bottom plate of the capacitor array with Analog_In or $V_{R E F}$. These switches have a $\frac{P M O S w i d t h}{N M O S w i d t h}$ ratio of $\frac{25 \mu \mathrm{m}}{12.5 \mu \mathrm{m}}$, as they drive the largest capacitance, and by increasing the transistor sizes, the switches' ON resistance is reduced. Figure 5.23 shows that the switch ON resistance is reduced when larger sizes of transistors are used and when a higher supply voltage is used. Table 5.5 shows the settling times for charging the various capacitors in the capacitor array to $\operatorname{Analog} G N D$, or $\frac{1}{2} V_{D D}$, with their respective switches, at a supply voltage of $0.6 \mathrm{~V}$. The settling time for the capacitor charge to reach $95 \%$ of the desired potential was considered. 


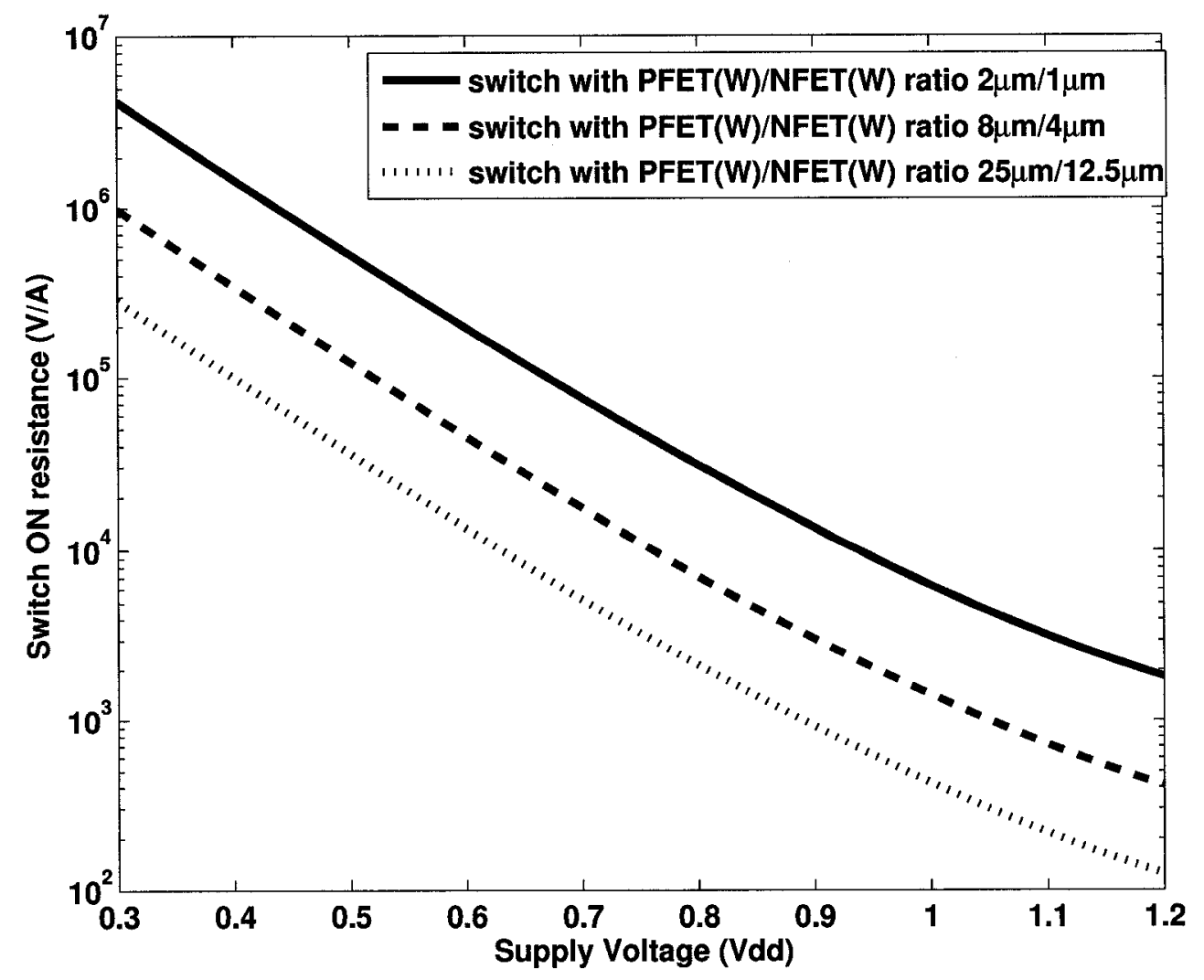

Figure 5.23: The three switches' ON resistance at various supply voltages.

Table 5.5: Settling time for charging each bit capacitor at $V_{D D}=0.6 \mathrm{~V}$.

\begin{tabular}{|l|c|c|c|c|}
\hline NMOS Size & PMOS Size & Maximum $R_{O N}\left(\frac{V}{A}\right)$ & Load Capacitor & Settling Time \\
\hline \hline$\frac{W}{L}=\frac{2 \mu m}{0.12 \mu m}$ & $\frac{W}{L}=\frac{1 \mu m}{0.12 \mu m}$ & $196 k \Omega$ & $440 \mathrm{fF}$ & $304 \mathrm{~ns}$ \\
\hline$\frac{W}{L}=\frac{2 \mu m}{0.12 \mu m}$ & $\frac{W}{L}=\frac{1 \mu m}{0.12 \mu m}$ & $196 k \Omega$ & $880 \mathrm{fF}$ & $607 \mathrm{~ns}$ \\
\hline$\frac{W}{L}=\frac{2 \mu m}{0.12 \mu m}$ & $\frac{W}{L}=\frac{1 \mu m}{0.12 \mu m}$ & $196 k \Omega$ & $1.760 \mathrm{pF}$ & $1.2 \mathrm{ps}$ \\
\hline$\frac{W}{L}=\frac{8 \mu m}{0.12 \mu m}$ & $\frac{W}{L}=\frac{4 \mu m}{0.12 \mu m}$ & $45 k \Omega$ & $3.520 \mathrm{pF}$ & $566 \mathrm{~ns}$ \\
\hline$\frac{W}{L}=\frac{8 \mu m}{0.12 \mu m}$ & $\frac{W}{L}=\frac{4 \mu m}{0.12 \mu m}$ & $45 k \Omega$ & $7.040 \mathrm{pF}$ & $1.13 \mathrm{ps}$ \\
\hline
\end{tabular}




\subsubsection{Design of the Output Buffers for the SAR ADC}

The output from the successive approximation register shows the digital bits as they go through a cycle including each bit going high for the first clock cycle, then going low, followed by once again going high until conversion is done for each bit. After conversion, each bit settles to either 1 or 0 depending on the analog input voltage. In order to only output the relevant data, the SAR output bits are passed through an output buffer that will only output the digital bits when the data is valid. After conversion is complete, the Control signal is triggered. This signal can be used to drive the output buffers in order to only output valid data after conversion is complete. The circuit in Figure 5.24 shows the output buffer design. The flip-flop only passes data when it is found to be valid using the Control signal, and then passes it to sequential inverters, or a 2-stage CMOS buffer.

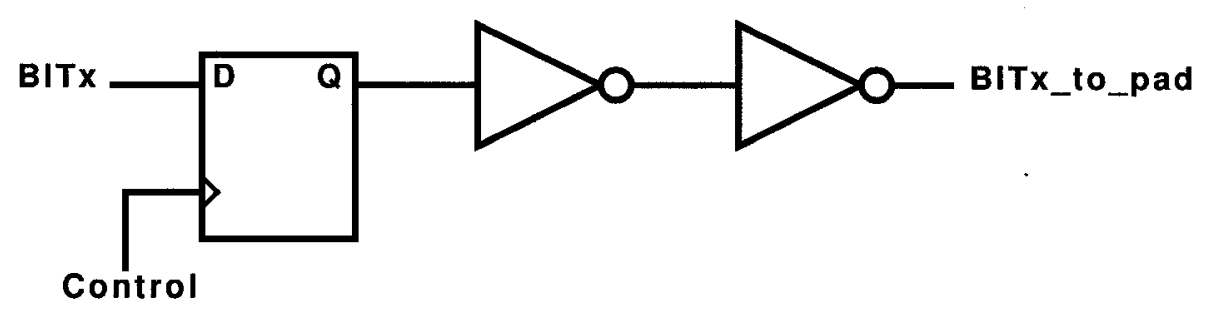

Figure 5.24: Schematic showing the output buffer driven by the Control signal.

\subsection{0-bit Analog to Digital Converter with a Tun- able Capacitor Array}

The designed ADC uses an analog ground of $\frac{1}{2} V_{D D}$, which allows the ADC to deal with inputs between $\frac{V_{D D}}{2}$ and $V_{D D}$. Because of the way the successive approximation is performed, the top plate of the capacitor array will vary between $0 \mathrm{~V}$ and $0.75 V_{D D}$. 
An attempt to create a full swing successive approximation $\mathrm{ADC}$ would require a more complex circuit and higher power dissipation [18]. The capacitor array for a 10-bit ADC requires a large capacitance spread, with the largest capacitor sized up to 512 times the smallest capacitor. This would require too much power to charge and discharge the capacitors and too large a chip area. An attenuation capacitor was implemented in order to reduce the maximum capacitor to only 16 times the smallest capacitor. Because of the significant potential for variation during processing, the attenuation capacitor was designed to be tunable, allowing for a 5-bit digital input to select a capacitance post-fabrication.

Figure 5.25 shows the final layout for the full ADC. The largest area is taken by the capacitor array with the tunable attenuation capacitor, followed by the SAR and control digital circuit. The full area of the chip, including pads and ESD protection has the length by width dimensions of $1.25 \mathrm{~mm}$ by $1.25 \mathrm{~mm}$. A description of the pins used for the ADC are shown in Table 5.6.

\subsection{ADC Design Summary}

The schematic for the comparator is shown in Figure 5.3. It uses two stages, the first of which is a differential amplifier, followed by an inverter. The second stage is an offset voltage correction circuit, used to reduce the offset voltage caused due to processing and device mismatch variation. The addition of the second stage improved the yield, after processing and mismatch errors, from $14.5 \%$ to $73.2 \%$. The dimensions of the comparator layout are $71 \mu m$ by $47 \mu m$, shown in Figure 5.7. 


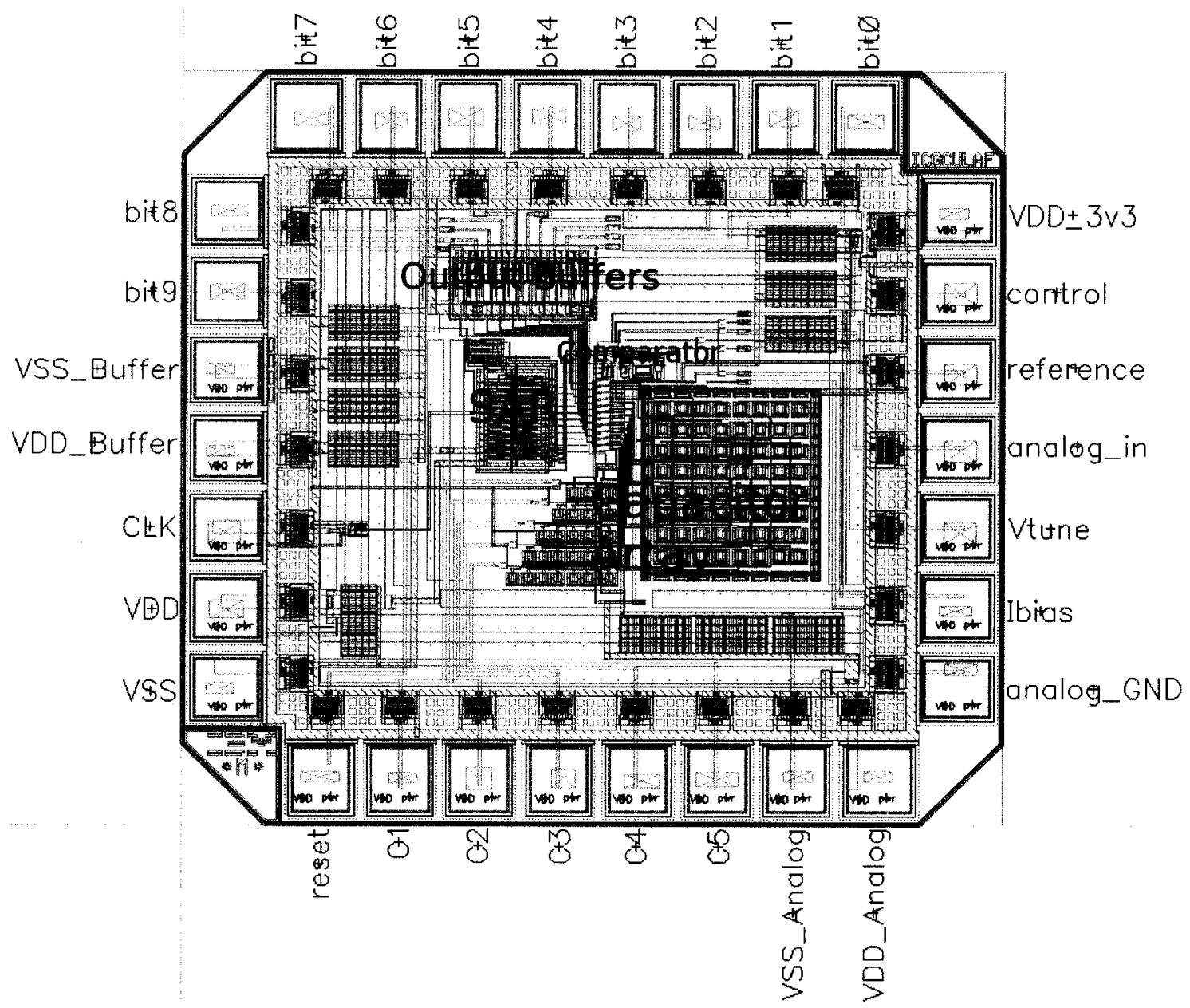

Figure 5.25: Complete layout of the designed ADC with dimensions of $1.25 \mathrm{~mm} \mathrm{x}$ $1.25 \mathrm{~mm}$. 
Table 5.6: Summary of the ADC pins.

\begin{tabular}{|c|c|}
\hline Pin Name & Pin Function \\
\hline \hline bit0 & Digital output of bit 0, either at 0V or at $V_{D D}$ \\
\hline bit1 & Digital output of bit 1, either at 0V or at $V_{D D}$ \\
\hline bit2 & Digital output of bit 2, either at 0V or at $V_{D D}$ \\
\hline bit3 & Digital output of bit 3, either at 0V or at $V_{D D}$ \\
\hline bit4 & Digital output of bit 4 , either at 0V or at $V_{D D}$ \\
\hline bit5 & Digital output of bit 5 , either at 0V or at $V_{D D}$ \\
\hline bit6 & Digital output of bit 6, either at 0V or at $V_{D D}$ \\
\hline bit7 & Digital output of bit 7, either at 0V or at $V_{D D}$ \\
\hline bit8 & Digital output of bit 8, either at 0V or at $V_{D D}$ \\
\hline bit9 & Digital output of bit 9, either at 0V or at $V_{D D}$ \\
\hline VSS_Buffer & Ground voltage for the output buffers $(0 \mathrm{~V})$ \\
\hline VDD_Buffer & Supply voltage for the output buffers \\
\hline CLK & clock for the digital circuitry (14 x bandwidth) \\
\hline VDD & Supply voltage for the digital SAR circuit \\
\hline VSS & Ground voltage for the digital SAR circuit (0V) \\
\hline reset & Active LOW reset for the digital logic \\
\hline C1 & Digital control bit 1 for the tunable attenuation capacitor \\
\hline C2 & Digital control bit 2 for the tunable attenuation capacitor \\
\hline C3 & Digital control bit 3 for the tunable attenuation capacitor \\
\hline C4 & Digital control bit 4 for the tunable attenuation capacitor \\
\hline C5 & Digital control bit 5 for the tunable attenuation capacitor \\
\hline VSS_Analog & Ground voltage for the analog circuit components (0V) \\
\hline VDD_Analog & Supply voltage for the analog circuit components \\
\hline Analog_GND & Reference to analog ground, at $V_{D D} / 2$ \\
\hline Ibias & Used to set the current bias for the comparator \\
\hline Vtune & Used to adjust the offset voltage of the comparator (set to $\left.V_{D D} / 2\right)$ \\
\hline Analog_In & Analog input to the ADC, between $V_{D D} / 2$ and $V_{D D}$ \\
\hline Reference & Alternative input to the ADC for test purposes only \\
\hline Control & Control signal for a high-voltage switch used for test purposes only \\
\hline VDD_3v3 & Supply voltage for the high-voltage switch used for test purposes only \\
\hline
\end{tabular}


The successive approximation register is composed of standard CMOS gates, including NAND gates, NOR gates, and inverters. The gates were designed with power optimization in mind, with little concern for speed, since it was not expected that the speed of the digital logic will be the bottleneck of the ADC. The SAR works as a shift register where at each clock cycle the output from the comparator is propagated to the appropriate output bit. the dimensions of the SAR layout and the reset control circuitry are $205 \mu \mathrm{m}$ by $150 \mu \mathrm{m}$.

The binary weighted capacitor array was designed using an attenuation capacitor in order to reduce the total capacitor area requirement. The ideal attenuation capacitor value is $647 \mathrm{fF}$, and it was designed to be variable, with values between $572 \mathrm{fF}$ and $694 \mathrm{fF}$, selected using 5 digital input bits. The capacitor array was laid out using a common centroid layout in order to reduce capacitor mismatch due to cross-chip gradients. The total area of the capacitor array, with the variable attenuation capacitor was $290 \mu m$ by $230 \mu m$.

The complete layout of the designed ADC is shown in Figure 5.25, with dimensions of $1.25 \mathrm{~mm} \times 1.25 \mathrm{~mm}$. The ADC has 30 pins, of which 10 are the digital output bits and 5 are variable attenuation capacitor digital input bits. All the bits are identified in Table 5.6. 


\section{Chapter 6}

\section{Simulation Results of the ADC}

\subsection{Linearity Simulated Results}

The linearity results presented include the effects of parasitic capacitances and bond pads, extracted from the layout in Figure 5.25. The variable attenuation capacitor, shown in Figure 5.19, was set to its designed optimum value of '10000' for $C_{1} C_{2} C_{3} C_{4} C_{5}$. DNL is the difference between an ideal 1 LSB and the worst case actual input voltage change between output code transitions, shown in $6.1(\mathrm{a})$. A DNL error specification of less than or equal to 1LSB guarantees a monotonic transfer function with no missing codes [44]. INL error describes the difference between an ideal linear transfer curve for an ADC and it includes quantization error, offset error, and gain error. A sample measurement for INL is shown in Figure 6.1(b). The INL and DNL simulations were performed by having a slow ramp from analog ground, $\frac{V_{D D}}{2}$ to $V_{D D}$ at the ADC input.

A slow ramp that ranges from $0.3 \mathrm{~V}$ to $0.6 \mathrm{~V}$ in $140 \mathrm{~ms}$ was placed at the input while $V_{D D}$ is set to $0.6 \mathrm{~V}$. This results in 14000 samples, meaning each of the 1024 codes is sampled 14 times. Figure 6.2 shows the digital output versus the analog 


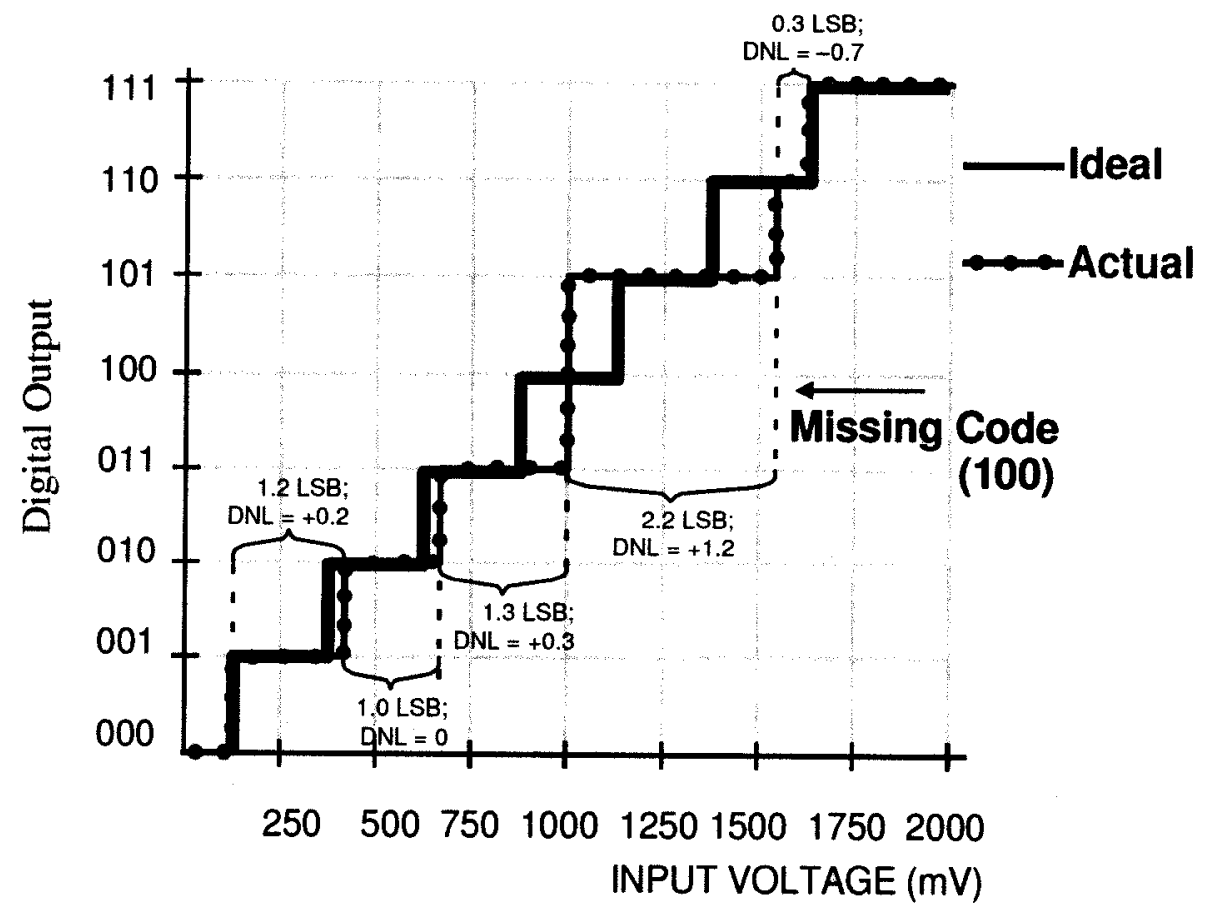

(a) Differential Non-Linearity (DNL).

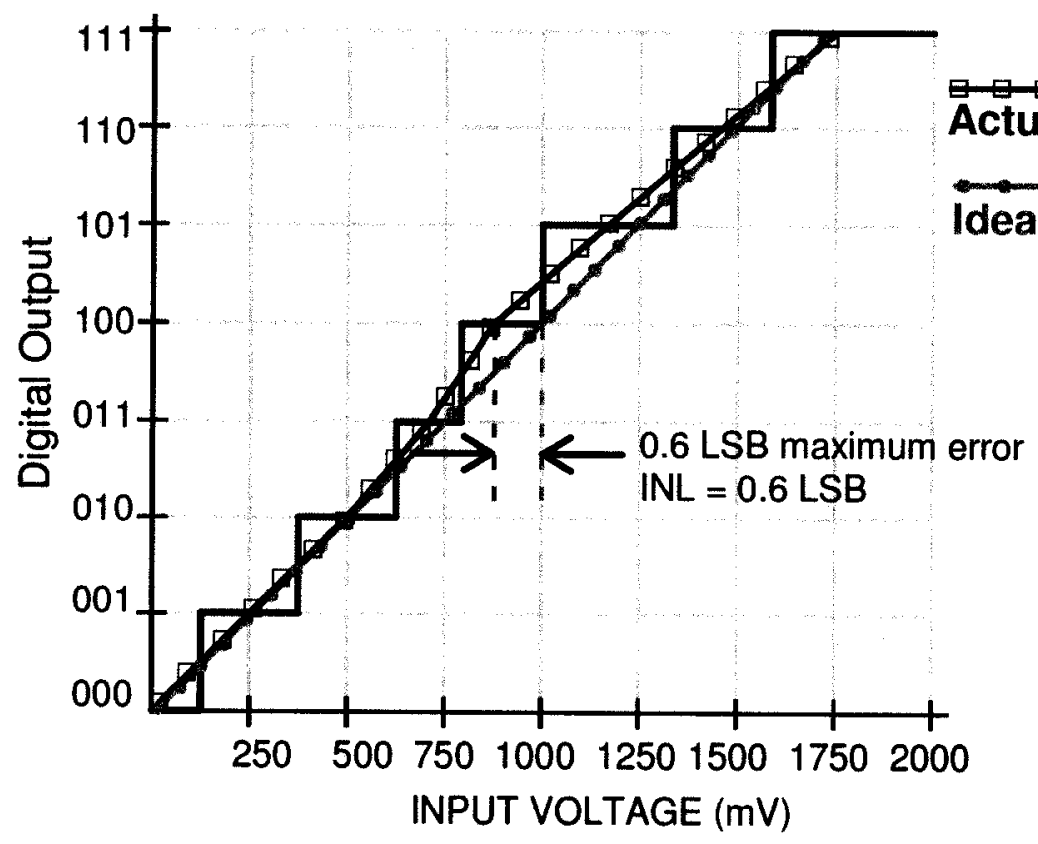

(b) Integral Non-Linearity (INL).

Figure 6.1: Sample DNL and INL measurements. 
input for the simulation, along with a best-fit line super-imposed. Using the line of best-fit for INL, maximum INL was found to be $0.26 \mathrm{LSB}$, with all codes present as shown in Figure 6.3. The figure also shows maximum DNL is 0.29LSB.

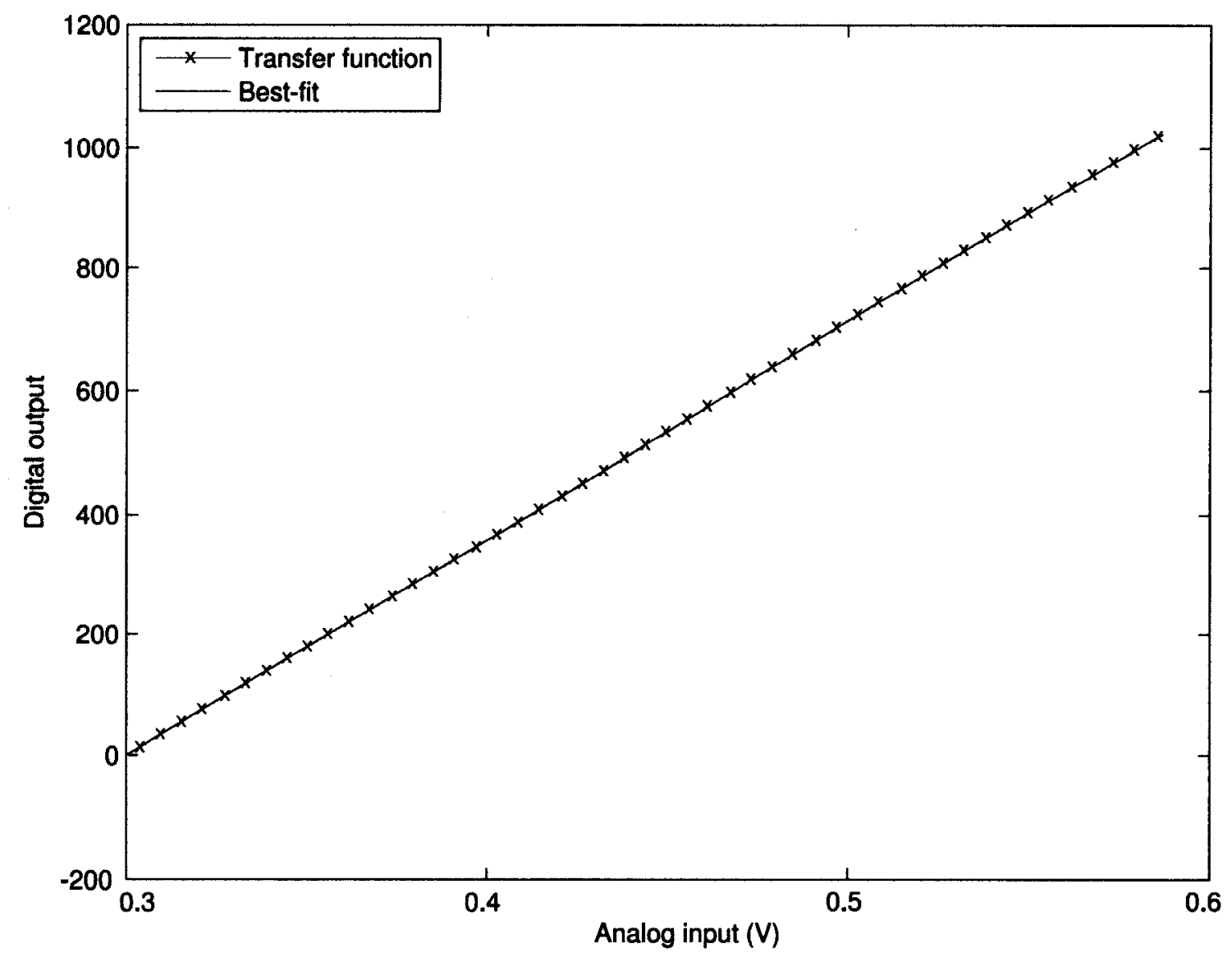

Figure 6.2: Digital output superimposed on the analog input for the ADC at $100 \mathrm{kS} / \mathrm{s}$ with a $V_{D D}=0.6 \mathrm{~V}$.

Similarly, simulations were performed at other voltage levels. Figure 6.4 shows the INL and DNL results when the ADC is running at $320 \mathrm{kS} / \mathrm{s}$, with a VDD of $1.0 \mathrm{~V}$. A slow ramp, from $0.5 \mathrm{~V}$ to $1.0 \mathrm{~V}$ in $45 \mathrm{~ms}$ was placed at the input in order to span the full 1024 codes. Figure 6.4 shows that INL is $0.16 \mathrm{LSB}$, while DNL is $0.29 \mathrm{LSB}$, with all codes present.

Figure 6.5 shows the INL and DNL results when the ADC is running at $500 \mathrm{kS} / \mathrm{s}$, 

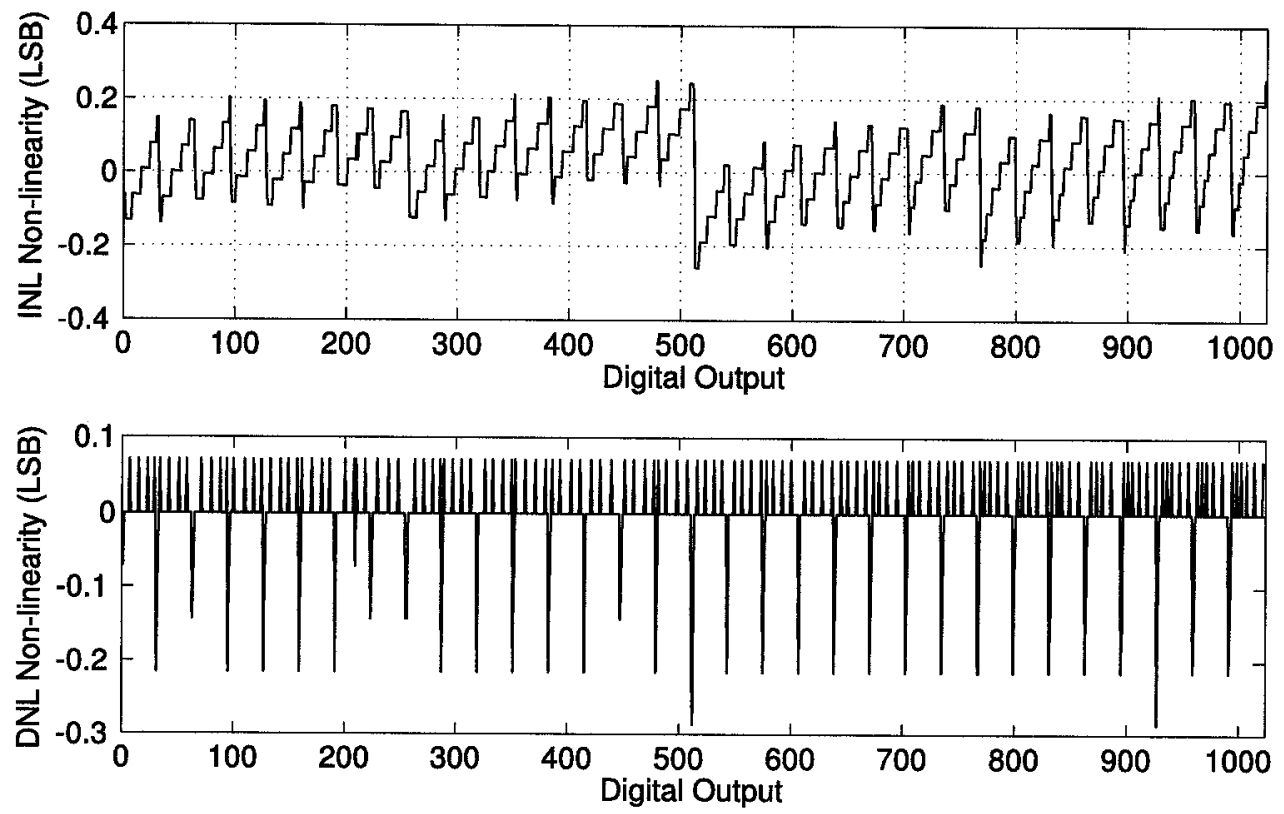

Figure 6.3: INL and DNL plots for all codes at $100 \mathrm{kS} / \mathrm{s}, \mathrm{VDD}=0.6 \mathrm{~V}$. 

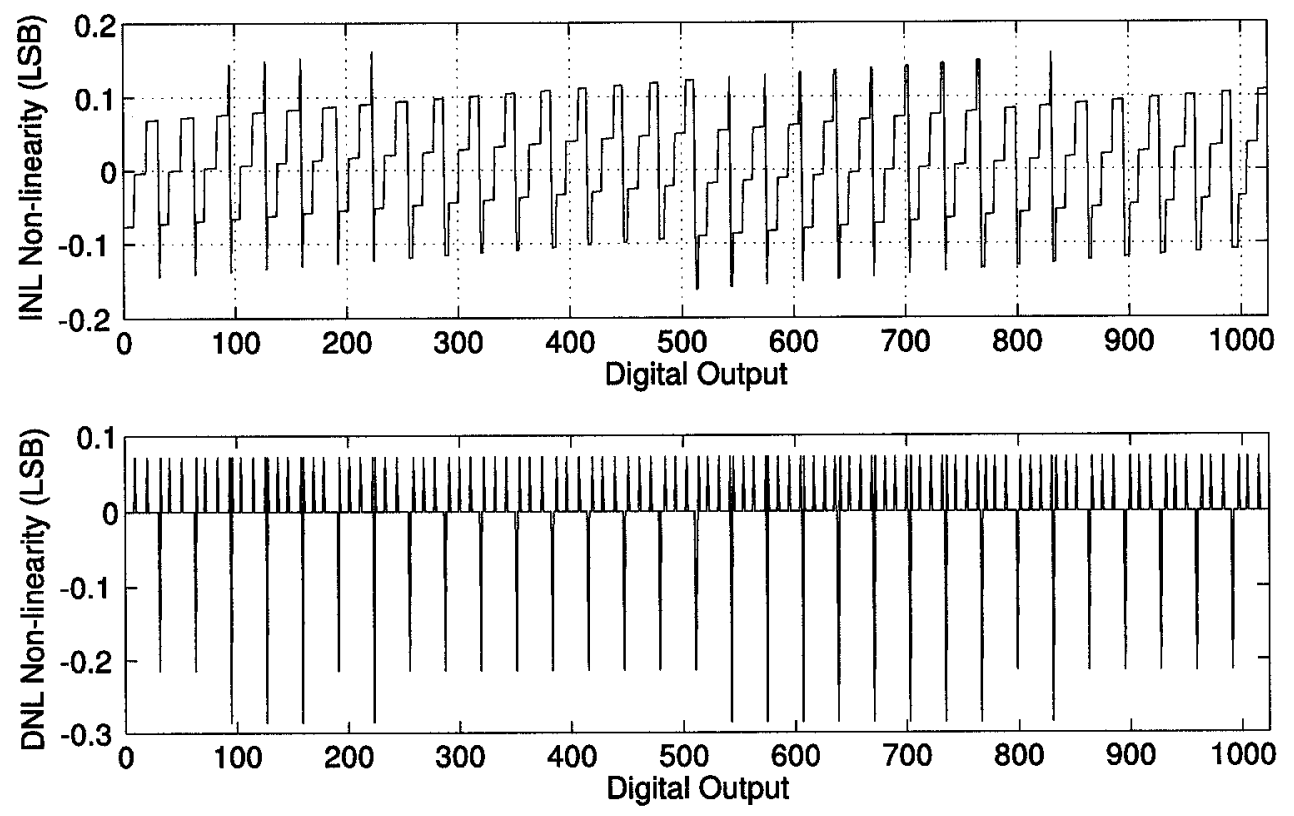

Figure 6.4: INL and DNL plots for all codes at $320 \mathrm{kS} / \mathrm{s}, \mathrm{VDD}=1.0 \mathrm{~V}$.

with a VDD of $1.2 \mathrm{~V}$. A slow ramp, from $0.6 \mathrm{~V}$ to $1.2 \mathrm{~V}$ in $29 \mathrm{~ms}$ was placed at the input in order to span the full 1024 codes. Figure 6.5 shows that INL is $0.10 \mathrm{LSB}$, while DNL is $0.14 \mathrm{LSB}$, with all codes present.

It is evident that, for the voltage supplies simulated, there is a period at which the non-linearity spikes occur. Observing Figure 6.3, there is a re-occurrence of the negative spikes at a specific interval. The interval at which these spikes are seen is when the capacitor array's lowest 5 bits change from '11111' to '00000'. At this exchange, the attenuation capacitor is involved in deciding the output. This suggests that the variable attenuation capacitor does not perfectly reconstruct the capacitor array with doubling capacitor values. Even with these spikes, however, the ADC exhibits an INL and DNL below 1 LSB, guaranteeing a monotonic transfer function with no missing codes. The most significant spike occurs at the middle exchange, 

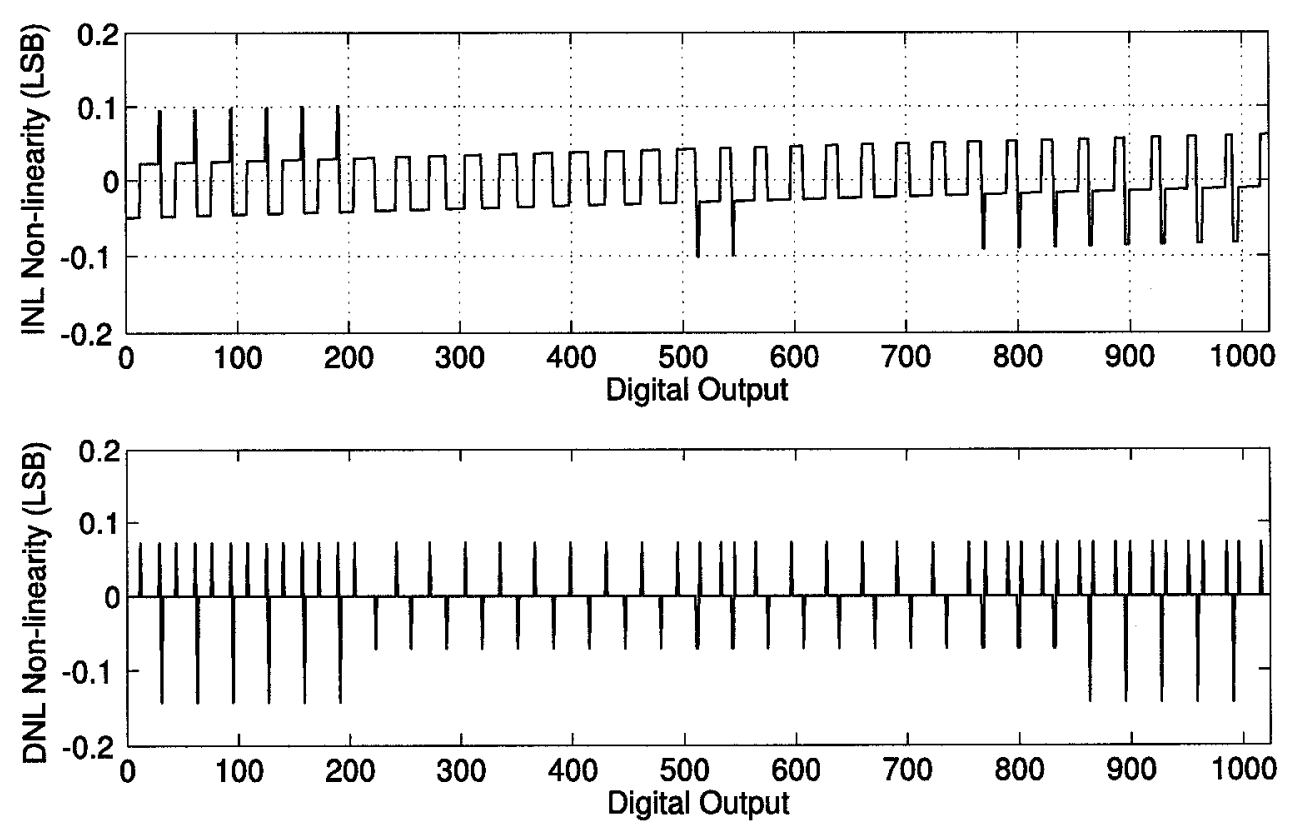

Figure 6.5: INL and DNL plots for all codes at $500 \mathrm{kS} / \mathrm{s}, \mathrm{VDD}=1.2 \mathrm{~V}$.

where the output of the ADC changes from '0111111111' to '1000000000'.

\subsubsection{ADC Attenuation Capacitor Tuning}

In order to find the optimal code for the tunable capacitor, INL and DNL measurements were taken for various capacitor ranges. Results are recorded in Figure 6.6. Figure 6.6 shows that " 10000 " is the optimal code, as the lowest INL and DNL were measured at this capacitor setting.

\subsection{Dynamic Simulated Results}

The dynamic performance of the ADC is measured by sending a sinusoidal input into the $\mathrm{ADC}$, and analyzing the output. The 10-bit output is passed through an ideal 10-bit DAC to convert the bits back to an analog voltage. This converted signal is then analyzed for signal to noise ratio (SNR), total harmonic distortion (THD), 


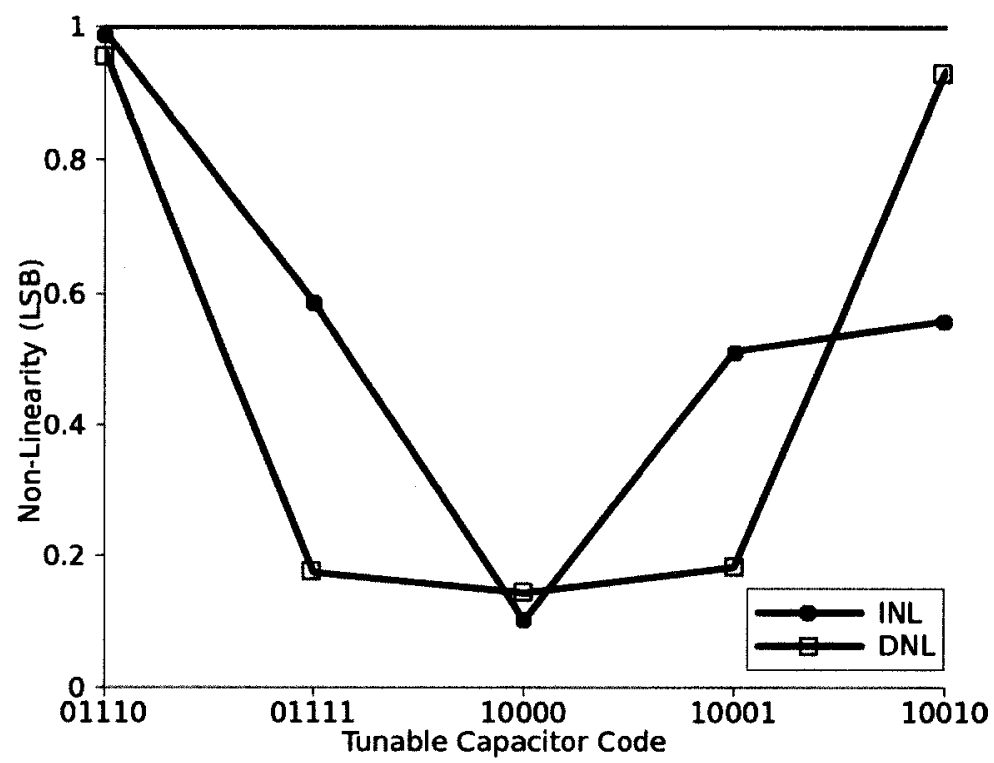

Figure 6.6: INL and DNL plots for all codes at $500 \mathrm{kS} / \mathrm{s}, \mathrm{VDD}=1.2 \mathrm{~V}$, Vref $=0.6 \mathrm{~V}$ spurious free dynamic range (SFDR), signal to noise and distortion ratio (SINAD), and effective number of bits (ENOB) [45]. The derivation of the dynamic evaluators is shown in Equations 6.1 to 6.5. These dynamic evaluators can be derived from a discrete Fourier transform (DFT) or a fast Fourier transform (FFT) plot of the output sinusoidal signal.

FFT analysis provides the frequency domain of the output signal. The FFT plot includes the fundamental frequency, harmonic frequencies, and noise at all other frequencies up to the Nyquist frequency. A simulation was performed using a sampling rate of $500 \mathrm{kHz}$ and a $V_{D D}$ of $1.2 \mathrm{~V}$ for an input sinusoidal signal at $200 \mathrm{~Hz}$. The output waveform is shown in Figure 6.7(a) and the FFT plot of this output is shown in Figure $6.7(\mathrm{~b})$.

SNR is the ratio between the fundamental signal power and the squared root of the square of the sum of all the noise in the Nyquist frequency range [2]. (Sample 


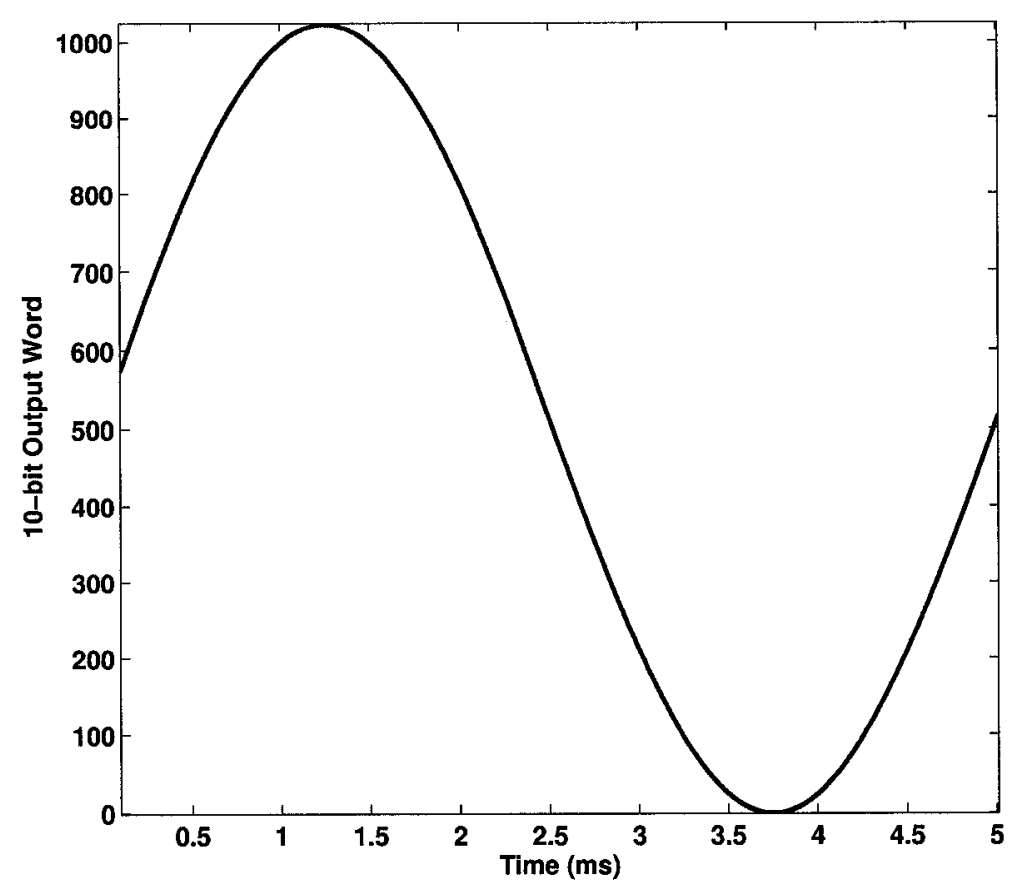

(a) $200 \mathrm{~Hz}$ sinusoid output in time domain.

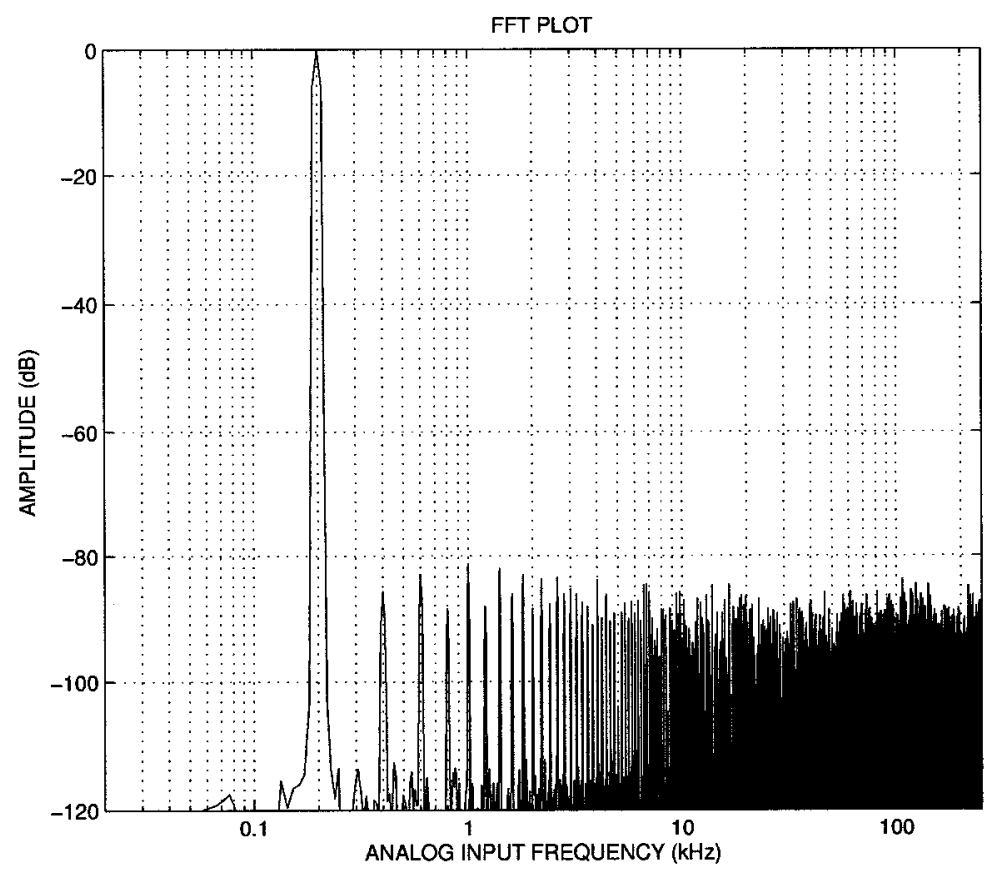

(b) $200 \mathrm{~Hz}$ sinusoid output in frequency domain.

Figure 6.7: Plot of the output from the ADC when a $200 \mathrm{~Hz}$ sinusoid is applied to the input, sampled at $500 \mathrm{kS} / \mathrm{s}$, with a $V_{D D}=1.2 \mathrm{~V}$. 
frequency / 2). Using Equation 6.1, this is found to be $61.43 \mathrm{~dB}$.

$$
S N R=20 \cdot \log \left(\frac{\text { Fundamental }}{\sqrt{\sum \text { Noise }^{2}}}\right) .
$$

The calculation for the THD, which characterizes the ratio of the sum of the harmonics to the fundamental signal [2], is found to be $-77.82 \mathrm{~dB}$. The equation for the THD is shown in Equation 6.2.

$$
T H D=20 \cdot \log \left(\frac{\sqrt{\sum \text { Harmonics }^{2}}}{\text { Fundamental }}\right) .
$$

The SFDR is found by evaluating the difference between the fundamental signal and the highest spurious noise in the spectrum [2], and is found to be $84.03 \mathrm{~dB}$.

$$
S F D R=20 \cdot \log \left(\frac{\text { Fundamental }}{\text { HighestSpurs }}\right)
$$

SINAD is a combination of the SNR and THD [2], evaluated to be $61.33 \mathrm{~dB}$ using Equation 6.4.

$$
S I N A D=20 \cdot \log \left(\frac{\text { Fundamental }}{\sqrt{\sum(\text { Noise }+ \text { Harmonics })^{2}}}\right) .
$$

The Effective number of bits is calculated using the SINAD and Equation 6.5. It is found to be 9.9 bits for the simulation.

$$
E N O B=\frac{S I N A D-1.76}{6.02}
$$

Similar analysis to the above was performed for an input sinusoidal signal of $20 \mathrm{~Hz}$, sampled at $100 \mathrm{kS} / \mathrm{s}$, with a $V_{D D}$ of $0.6 \mathrm{~V}$. In this case, the ADC is fully immersed in the subthreshold region of operation. The resulting output waveform is shown in Figure 6.8(a) and the FFT plot is shown in Figure 6.8(b). The dynamic simulated values are 
Table 6.1: Summary of ADC performance at $0.6 \mathrm{~V}$ and $1.2 \mathrm{~V} V_{D D}$.

\begin{tabular}{|c|c|c|}
\hline ADC Characteristic & $\mathbf{V}_{D D}=\mathbf{0 . 6 V}$ & $\mathbf{V}_{D D}=\mathbf{1 . 2 V}$ \\
\hline Sample Rate & $\mathbf{1 0 0} \mathbf{~ k S} / \mathbf{s}$ & $\mathbf{5 0 0} \mathbf{~ k S} / \mathbf{s}$ \\
\hline \hline INL & $0.26 \mathrm{LSB}$ & $0.10 \mathrm{LSB}$ \\
\hline DNL & $0.29 \mathrm{LSB}$ & $0.14 \mathrm{LSB}$ \\
\hline \hline SNR & $62.56 \mathrm{~dB}$ & $61.43 \mathrm{~dB}$ \\
\hline SFDR & $84.22 \mathrm{~dB}$ & $84.03 \mathrm{~dB}$ \\
\hline THD & $-68.6 \mathrm{~dB}$ & $-77.82 \mathrm{~dB}$ \\
\hline SINAD & $61.59 \mathrm{~dB}$ & $61.33 \mathrm{~dB}$ \\
\hline ENOB & $9.94 \mathrm{bits}$ & $9.90 \mathrm{bits}$ \\
\hline
\end{tabular}

summarized in Table 6.1. This table appears to suggest that the dynamic operation of the ADC deteriorates with increasing voltage supply since the ENOB improves when the voltage supply is reduced from $1.2 \mathrm{~V}$ to $0.6 \mathrm{~V}$. This is not the case since different sample rates are applied at the different voltage levels. Therefore, it cannot be assumed that the dynamic performance of the ADC improves with decreasing voltage supply.

\subsection{Power Dissipation}

The power dissipation of the analog and digital components was measured using the fully extracted chip with parasitic capacitance and resistance included, for various values of $V_{D D}$. The lowest power dissipation is observed when the ADC operates fully in the subthreshold region, with a $V_{D D}$ of $0.6 \mathrm{~V}$. Increasing the $V_{D D}$ results in a sharp increase in power dissipation. An increase in supply voltage from $0.6 \mathrm{~V}$ to $1.2 \mathrm{~V}$ resulted in approximate power dissipation increase of 200 times. Figure 6.9 shows the increase in power for the ADC with increasing $V_{D D}$. However, higher sample rates for the ADC are achievable at higher supply voltages. This is expected since increasing supply voltages increases the drain currents of the transistors in the designed ADC. 


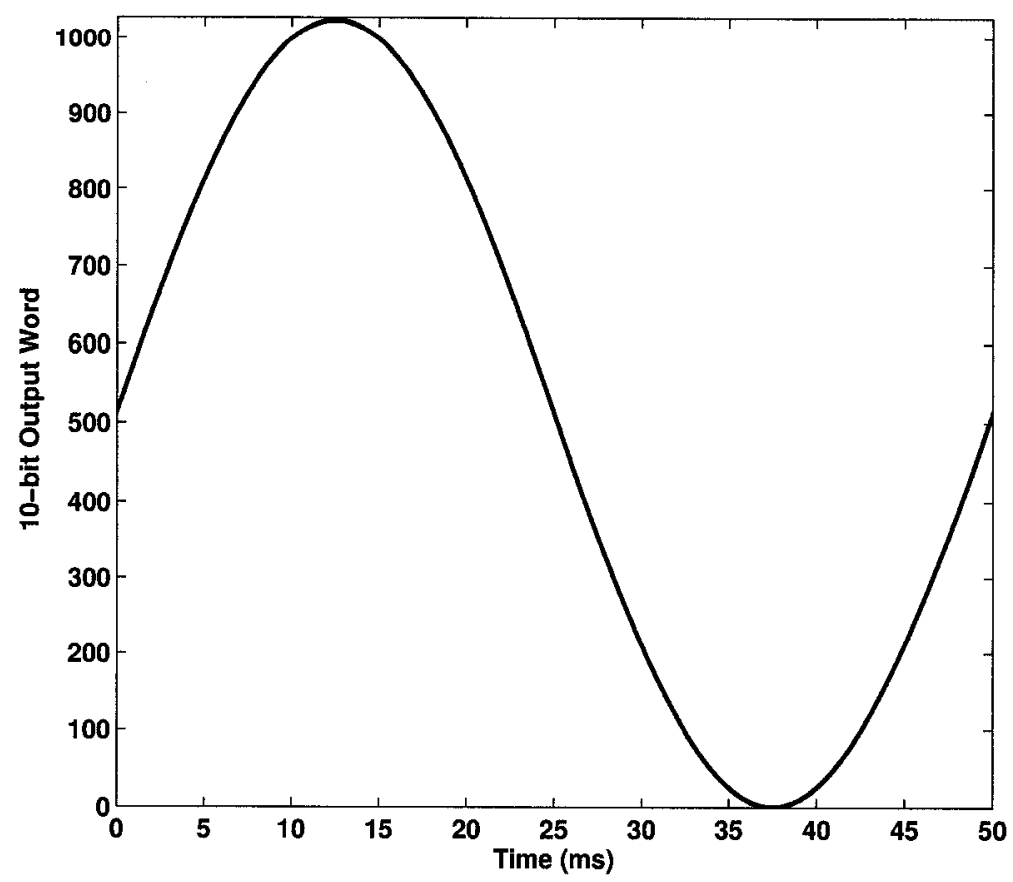

(a) $20 \mathrm{~Hz}$ sinusoid output in time domain.

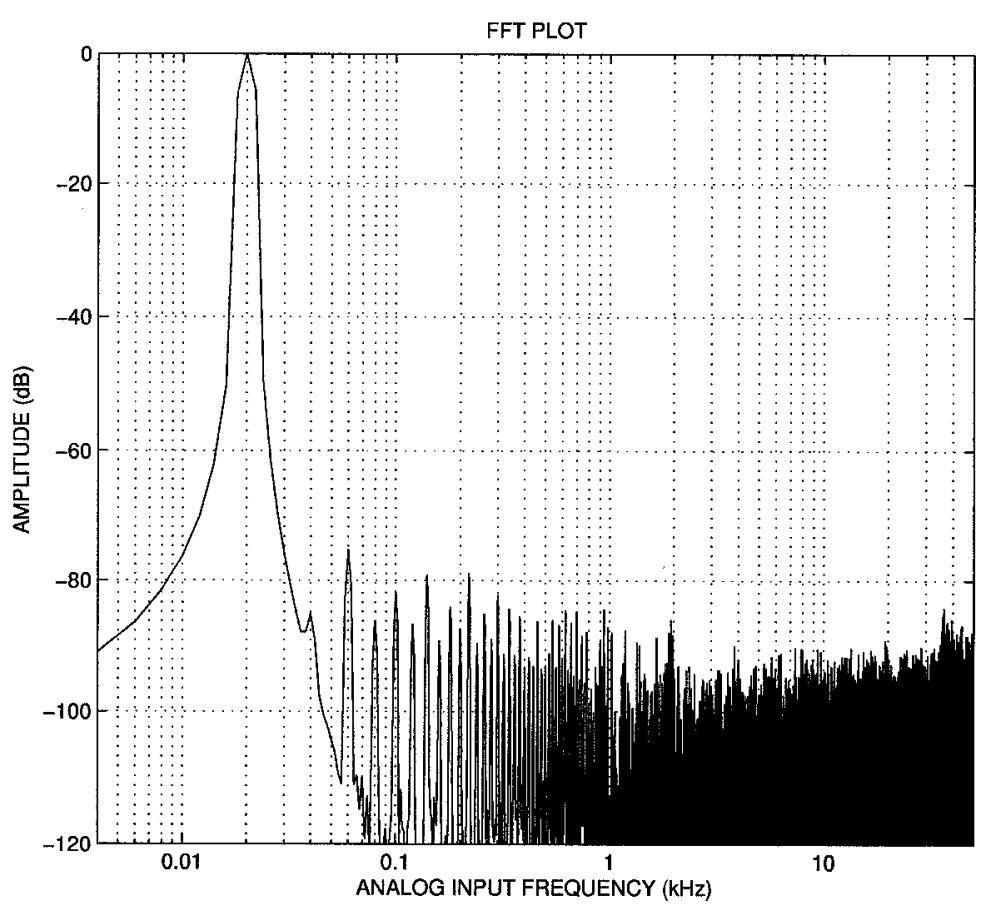

(b) $20 \mathrm{~Hz}$ sinusoid output in frequency domain.

Figure 6.8: Plot of the output from the ADC when a $20 \mathrm{~Hz}$ sinusoid is applied to the input, sampled at $100 \mathrm{kS} / \mathrm{s}$, with a $V_{D D}=0.6 \mathrm{~V}$. 
The speed of the ADC circuit is related to the transistor transconductance, which is directly proportional to the drain current of a transistor, from Equation A.8.

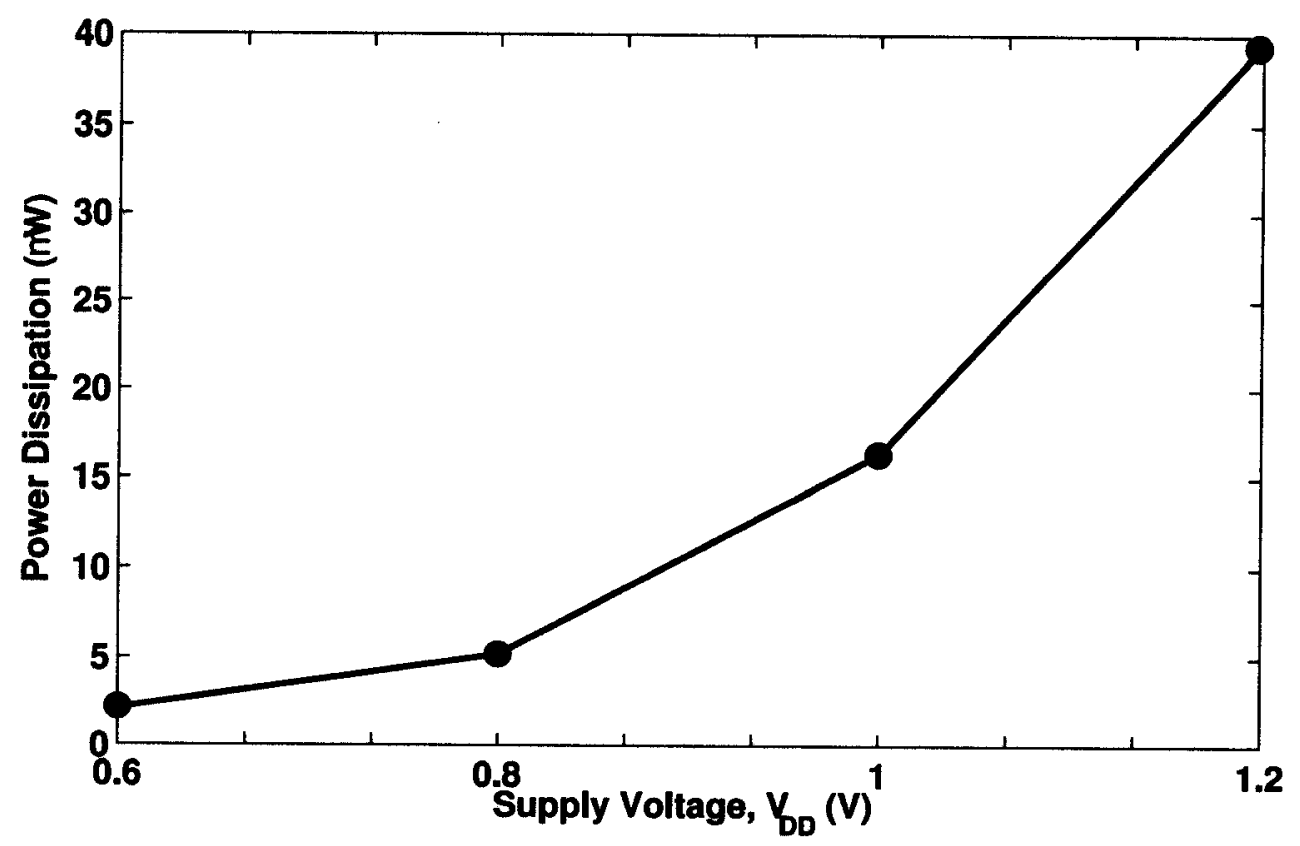

Figure 6.9: Power dissipation of the ADC with varying $V_{D D}$.

The minimum energy consumption per cycle is $20.9 \mathrm{pJ} /$ cycle, when the ADC is running at $100 \mathrm{kS} / \mathrm{s}$, with a $0.6 \mathrm{~V}$ power supply. By increasing the power supply, higher sample rates are achievable. Increasing the supply voltage increases the energy per cycle, but at a much slower rate when compared to the increase in power with increasing supply voltage. Figure 6.10 shows how the increase in energy per cycle for the ADC varies with varying $V_{D D}$.

Table 6.2 summarizes the power consumption for the ADC. Any further decrease in the supply voltage from $0.6 \mathrm{~V}$ degrades the bandwidth and dynamic performance of the ADC. 


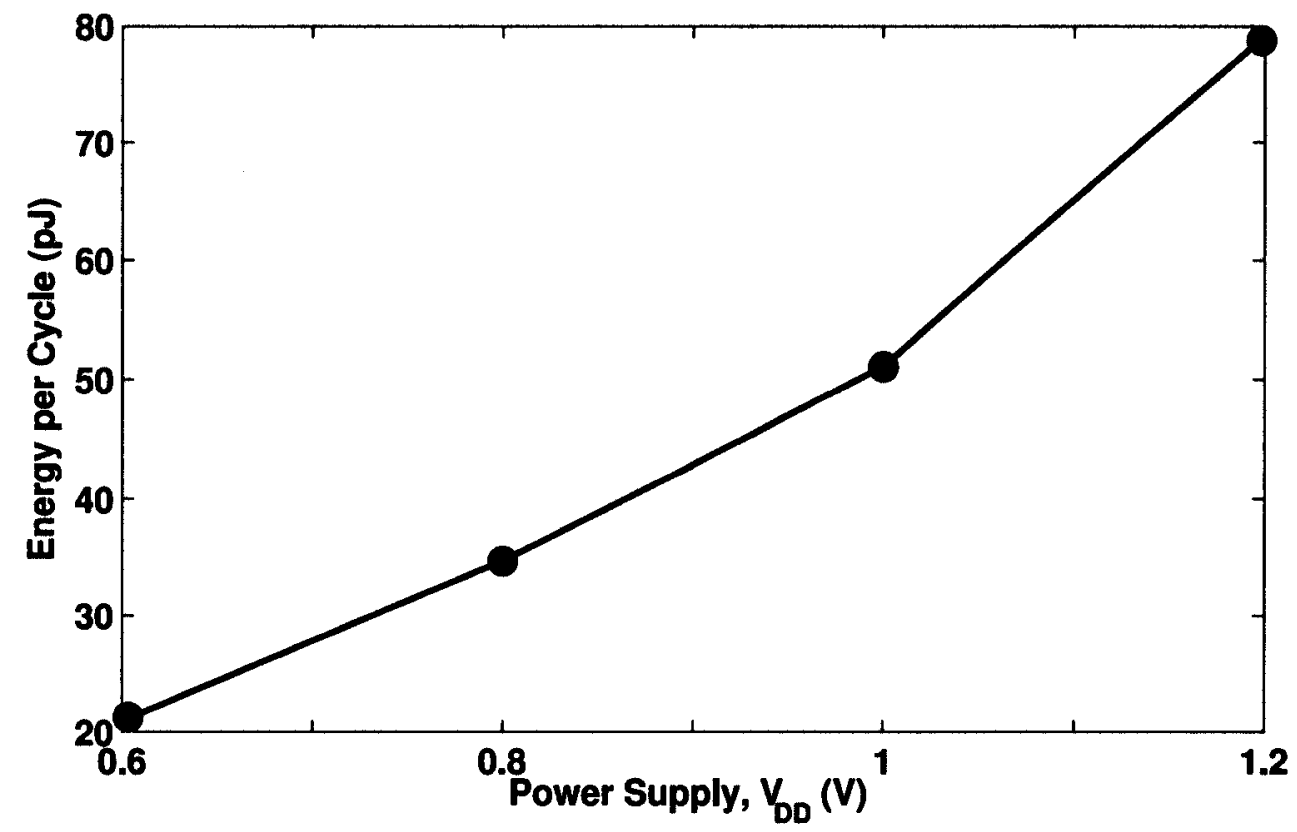

Figure 6.10: Energy per cycle of the ADC with varying $V_{D D}$.

Table 6.2: Summary of power consumption vs. $V_{D D}$

\begin{tabular}{|c|c|c|c|}
\hline$V_{D D}(V)$ & $\begin{array}{c}\text { Sample } \\
\text { Rate (kS/s) }\end{array}$ & $\begin{array}{c}\text { Power Consumption } \\
(\mu W)\end{array}$ & $\begin{array}{c}\text { Energy per Cycle } \\
(\mathbf{p J} / \text { cycle) }\end{array}$ \\
\hline 0.6 & 100 & 2.09 & 20.9 \\
\hline 0.8 & 150 & 5.21 & 34.73 \\
\hline 1.0 & 320 & 16.34 & 51.07 \\
\hline 1.2 & 500 & 39.56 & 79.12 \\
\hline
\end{tabular}




\subsection{Summary of Simulated Results}

In order to ensure that the simulations of the $\mathrm{ADC}$ meet the specifications, three types of simulations were required. The ADC linearity performance, dynamic performance, and power dissipation were simulated and analyzed. The simulations were performed using the complete ADC layout with bond pads, extracted to include parasitic capacitances.

The linearity was simulated with $V_{D D}=0.6 \mathrm{~V}, V_{D D}=1.0 \mathrm{~V}$ and $V_{D D}=1.2 \mathrm{~V}$, in order to find the INL and DNL of the ADC. The simulation involved placing a slow ramp that ranges from $\frac{1}{2} V_{D D}$ to $V_{D D}$ at the ADC input. When the voltage supply was set to $0.6 \mathrm{~V}$ and a sample rate of $100 \mathrm{kS} / \mathrm{s}$ was applied to the ADC, the INL and DNL were found to be $0.26 \mathrm{LSB}$ and $0.29 \mathrm{LSB}$, respectively. When the voltage supply was set to $1.0 \mathrm{~V}$, with a $320 \mathrm{kS} / \mathrm{s}$ sample rate, the INL and DNL were found to be $0.16 \mathrm{LSB}$ and $0.29 \mathrm{LSB}$, respectively. When the voltage supply was set to $1.2 \mathrm{~V}$, with a $500 \mathrm{kS} / \mathrm{s}$ sample rate, the INL and DNL were found to be $0.10 \mathrm{LSB}$ and $0.14 \mathrm{LSB}$, respectively. Tuning the attenuation capacitor showed that the optimal capacitor code is ' 10000 ' with linearity deteriorating when the capacitor is varied.

The dynamic performance of the ADC was measured by sending a sinusoidal input into the ADC, and analyzing the output. The characteristics that were analyzed were the SNR, THD, SFDR, SINAD, and ENOB. Simulations showed that the ADC operates with an ENOB of 9.94 , when $V_{D D}=0.6 \mathrm{~V}$ and the sample rate was $100 \mathrm{kS} / \mathrm{s}$. The ENOB was 9.90 when $V_{D D}=1.2 \mathrm{~V}$ and the sample rate was $500 \mathrm{kS} / \mathrm{s}$.

The power dissipation of the ADC was simulated, showing that the power increases 
significantly with increasing supply voltage, $V_{D D}$. However, because the sample rate can also be increased with increasing $V_{D D}$, the energy per cycle increased linearly with increasing $V_{D D}$. At $0.6 \mathrm{~V}$, and the $\mathrm{ADC}$ sampling at $100 \mathrm{kS} / \mathrm{s}$, the power dissipation of the $\mathrm{ADC}$ was $2.09 \mu \mathrm{W}$, corresponding to an energy per cycle of $20.9 \mathrm{pJ} /$ cycle. At $1.2 \mathrm{~V}$, and the ADC sampling at $500 \mathrm{kS} / \mathrm{s}$, the power dissipation of the $\mathrm{ADC}$ was $39.56 \mu W$, corresponding to an energy per cycle of $79.12 \mathrm{pJ} /$ cycle. 


\section{Chapter 7}

\section{Measured Results}

\subsection{Measurement Description}

The complete chip, including the core of the ADC design, the ESD protection, and external pins, was fabricated and is shown in Figure 7.1. The chip was subsequently placed in a QFN32 package and bonded using gold bond wires and a wedge bonder, shown in Figure 7.2(a). A PCB was designed in order to facilitate testing of the ADC. The basic test setup is shown in Figure 7.2(b), while a detailed explanation of the ADC measurement setup is described in Section 7.2. The main measurements of concern for the ADC will be the effects of capacitor tuning on the ADC's dynamic performance, and the ADC's power dissipation.

When measuring the dynamic performance of the ADC, four main measurements need to be taken into consideration, the SFDR, SNR, SINAD, and ENOB. These measurements will be derived by applying a sinusoid to the input of the ADC and observing the output frequency spectrum, with an FFT plot. A combination of the four measurements mentioned can provide us with a description of the ADC's performance. 


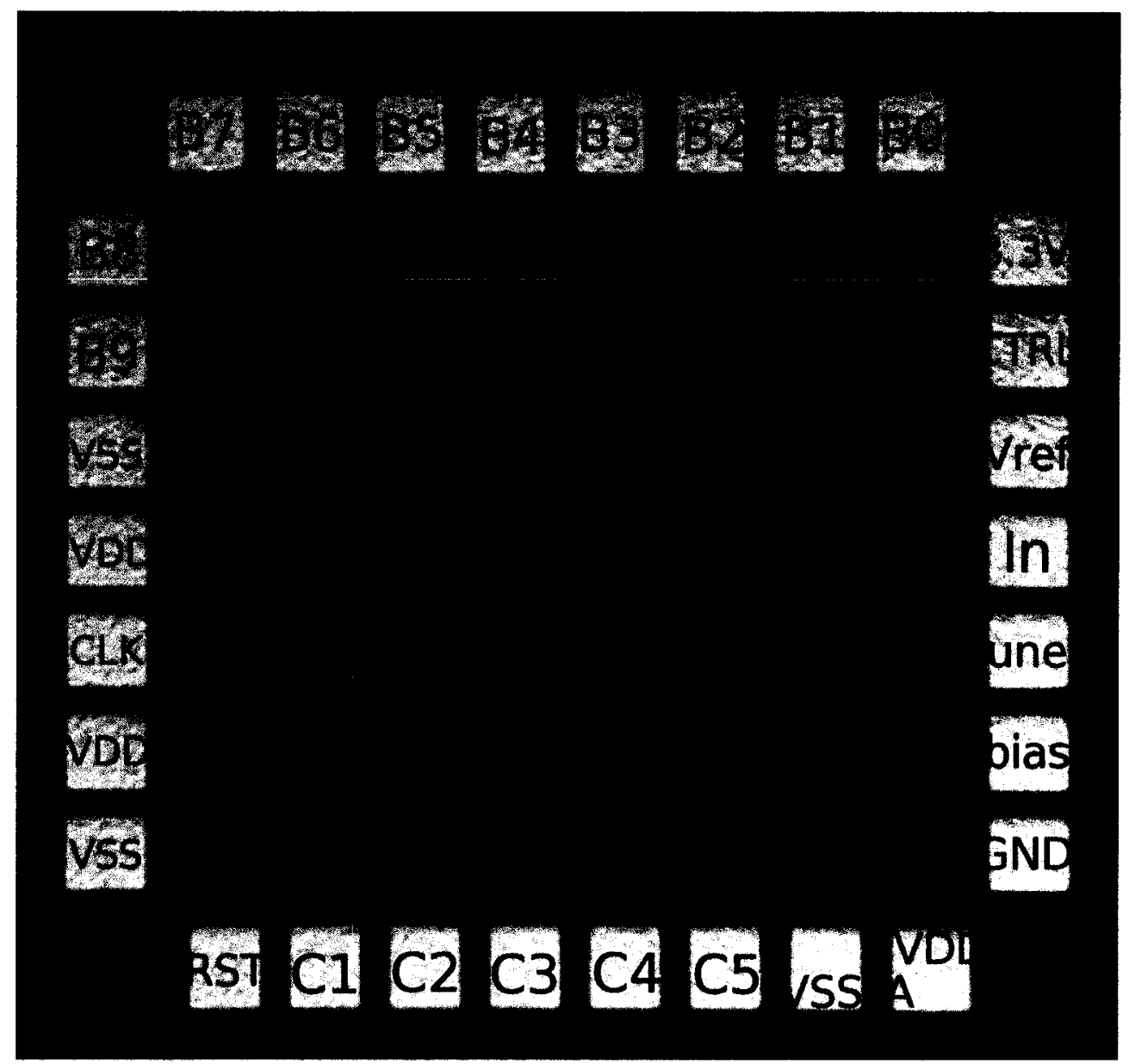

Figure 7.1: Complete fabricated ADC with pins labeled.

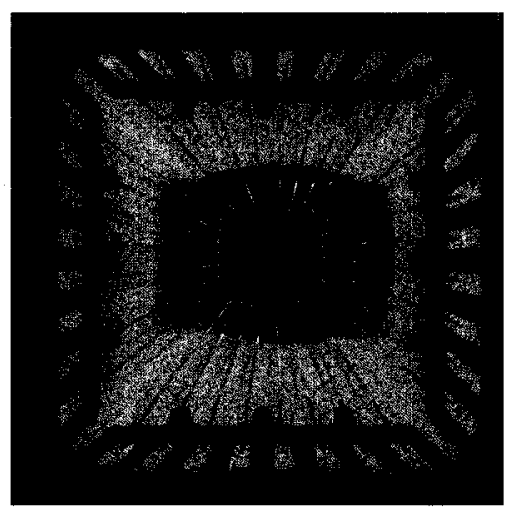

(a)

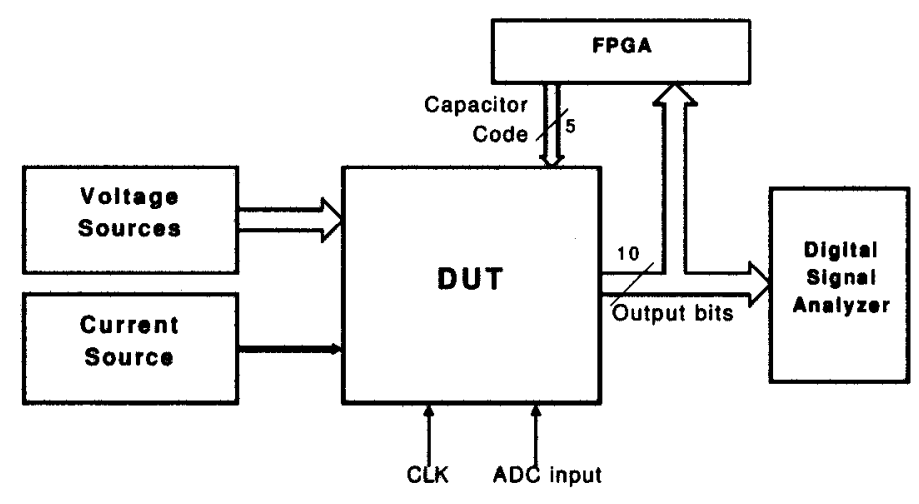

(b)

Figure 7.2: (a) Fabricated ADC bonded to QFN32 package and (b) Basic test setup for the ADC (DUT). 
The spurious free dynamic range (SFDR) is described in [2] as the difference between the value of the output signal at the desired frequency level and the value of the highest amplitude output frequency that is not found in the input. SFDR is presented in terms of $\mathrm{dB}$ below the fundamental frequency, shown in Figure 7.3.

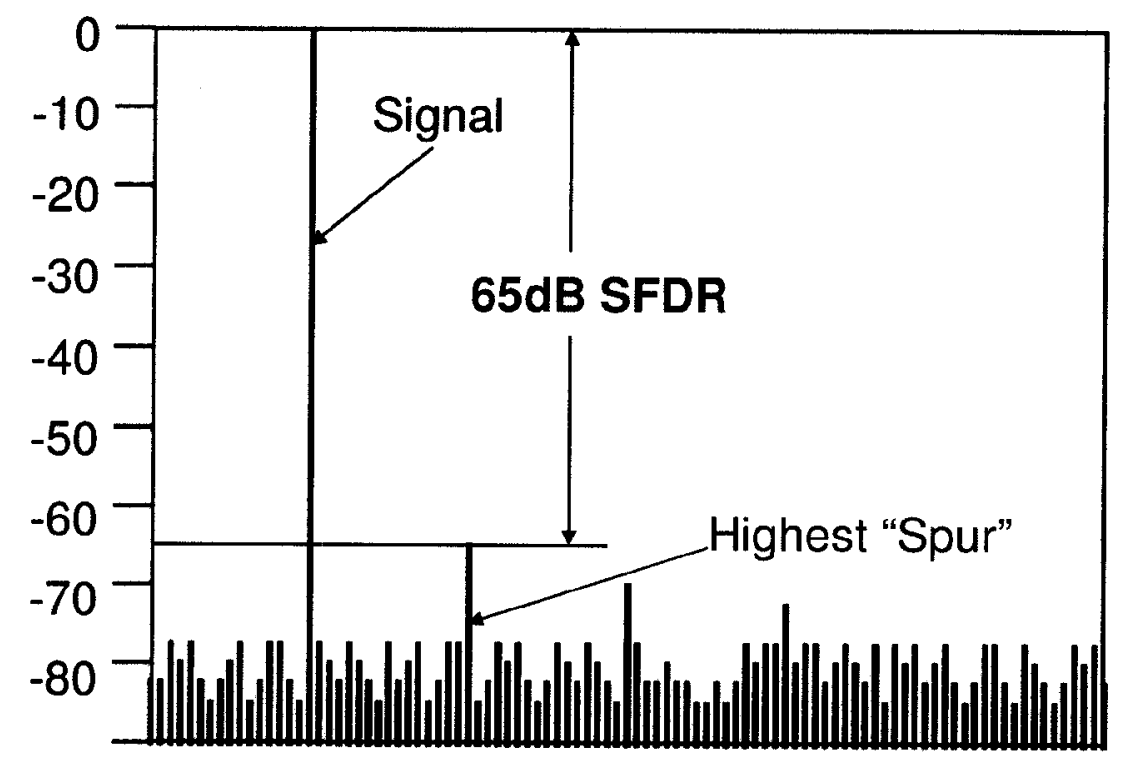

Figure 7.3: Sample SFDR measurement from an FFT plot [2].

The signal to noise ratio (SNR), as described in [2], is the ratio of the output signal amplitude to the noise level at the output, excluding harmonics and DC, shown in Figure 7.4. SNR is expected to increase with increasing input amplitude, until the maximum allowable input range is reached.

The signal to noise and distortion (SINAD) ratio, as described in [2], is an indicator of the dynamic performance of an ADC, which combines the effects of total harmonic distortion (THD) and SNR. It takes into consideration all the noise and harmonics below the Nyquist frequency, excluding DC. It is used as an overall measure of ADC dynamic performance. The THD is an indication of the ADC's linearity in terms of the harmonic content of a signal [2]. A pure sine wave has only one frequency 


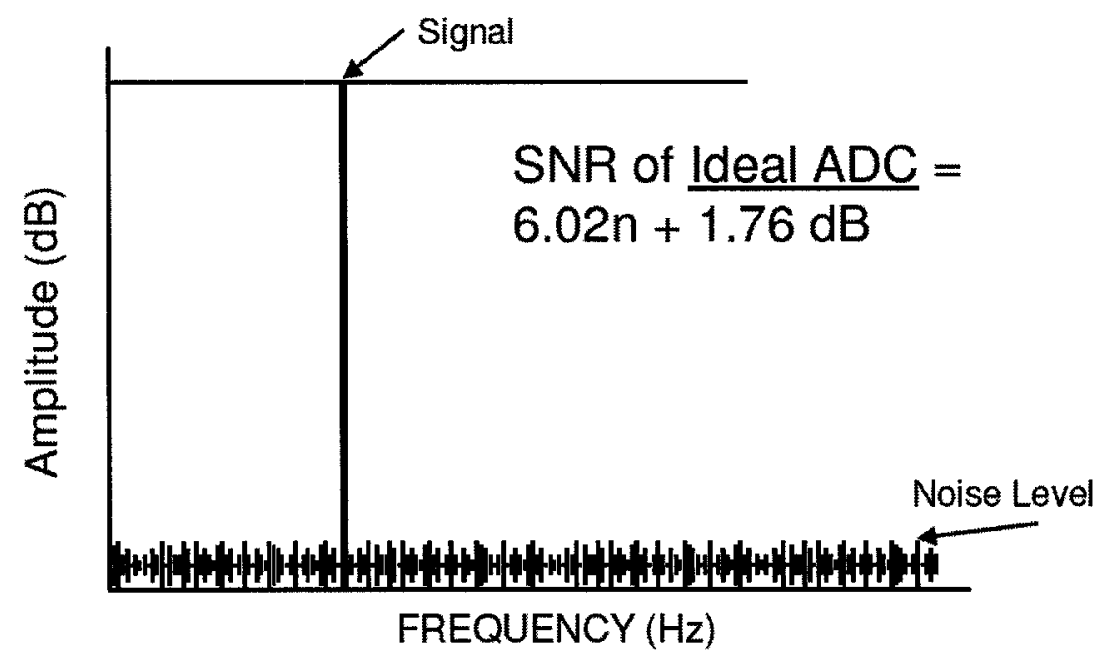

Figure 7.4: Sample SNR measurement from an FFT plot [2].

component, while any non-linearities in a converter's transfer function will introduce new harmonics at the output of the ADC. The potential effects of harmonic distortion due to non-linearities in an ADC are shown in Figure 7.5.
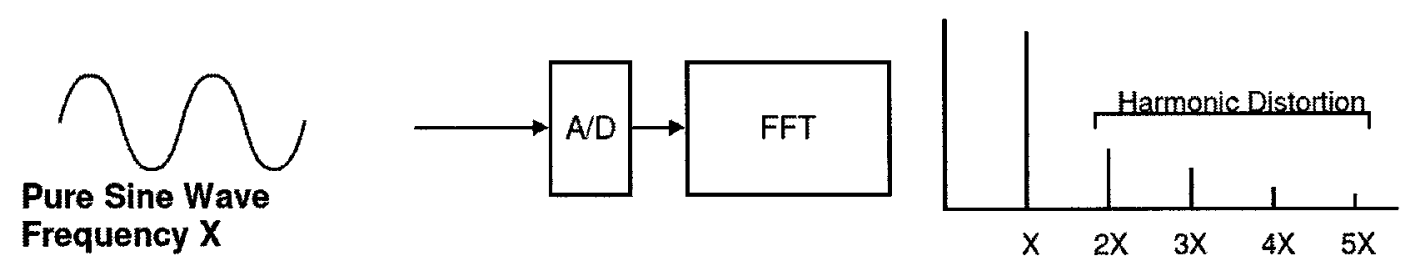

Figure 7.5: Effects of non-linearities in an A/D converter [2].

The effective number of bits (ENOB) is an extension of SINAD, as it is a calculation that quantifies the dynamic performance of the ADC. It compares the converter under test to a theoretically perfect converter with a resolution of ENOB. The equation that derives ENOB from SINAD is

$$
E N O B=\frac{S I N A D-1.76}{6.02}
$$




\subsection{Measurement Setup}

A micrograph of the manufactured chip is shown in Figure 7.1, with the pins labeled, while the test setup is shown in Figure 7.6. For an explanation of the pins, refer to Table 5.6. The chip was bonded to a QFN32 package using gold bond wires and a wedge bonder, and placed on a PCB. The layout for the PCB components is shown in Figure 7.7 and a labeled photograph of the PCB is shown in Figure 7.8. The dimensions of the designed PCB are $100 \mathrm{~mm} \times 100 \mathrm{~mm}$. The PCB was designed to include decoupling capacitors for all DC voltage supply pins, including VDD_Buffer, VDD_Analog, VDD_Digital, analog_GND, Ibias, Vtune, reference, and VDD_3V3. Decoupling capacitors were used to isolate the $\mathrm{AC}$ signal noise from the required $\mathrm{DC}$ power supply. These DC voltage pins, along with the output bits were connected to electrostatic discharge (ESD) protection, provided on the PCB. ESD is a sudden electric current that can flow into the chip on contact with a statically charged object, causing potentially high, unwanted currents that can cause damage to the fabricated chip. The CLK and analog_in signals were connected through sub-miniature type A (SMA) connectors on the PCB, while the reset, control, and capacitor selection pins (C1 to $\mathrm{C} 5)$ were connected through a dual in-line package (DIP) switch. The output bits were connected to an output header to accommodate the digital cable for the Agilent Infiniium 54832D MSO, as well as to a Mictor SB connector in order to accommodate the Xilinx Virtex II FPGA demo board cable.

The Xilinx Virtex II FPGA board was connected to the ADC's output bits and the capacitor code control bits. The Xilinx FPGA, along with a personal computer (PC) 


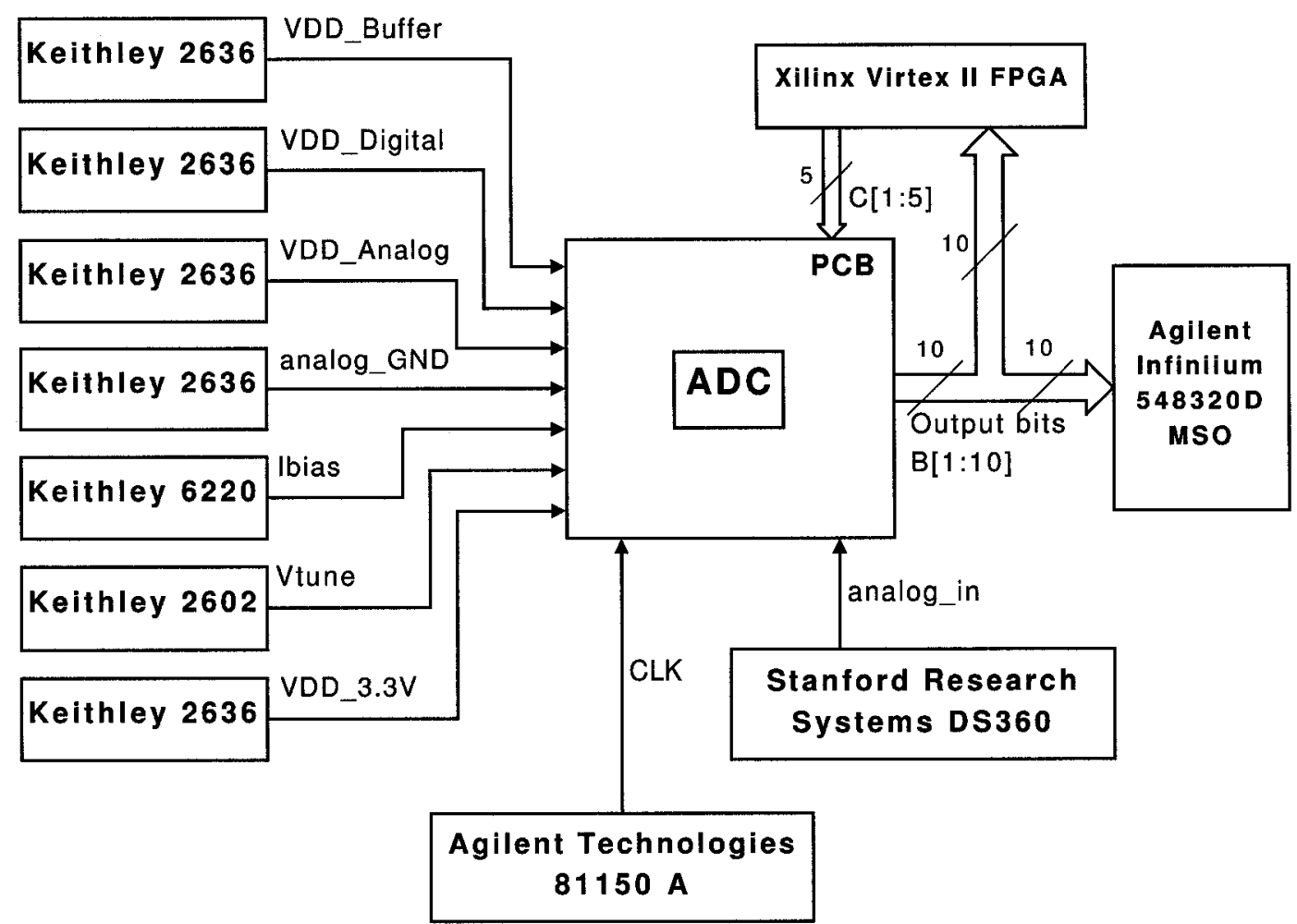

Figure 7.6: Test setup for the ADC.

running the MATLAB software tool, was used to control the attenuation capacitance.

The tested input to the ADC was a sine wave, ranging from $\frac{1}{2} V_{d d}$ to $V_{d d}$. For each capacitor code, the FPGA reads in $64 \mathrm{kB}$ of data from the 10-bit output, read in as 16-bit words. This data was then analyzed in MATLAB and the SINAD and ENOB were found for each capacitor code. The capacitor code that corresponds to the highest SINAD and ENOB was then kept as the operating attenuation capacitor code of the ADC.

\subsection{Dynamic Measured Results}

The dynamic performance of the ADC was measured when the supply voltage was set to $0.4 \mathrm{~V}, 0.6 \mathrm{~V}, 0.8 \mathrm{~V}, 1.0 \mathrm{~V}$, and $1.2 \mathrm{~V}$. This was done by applying a sinusoid input 


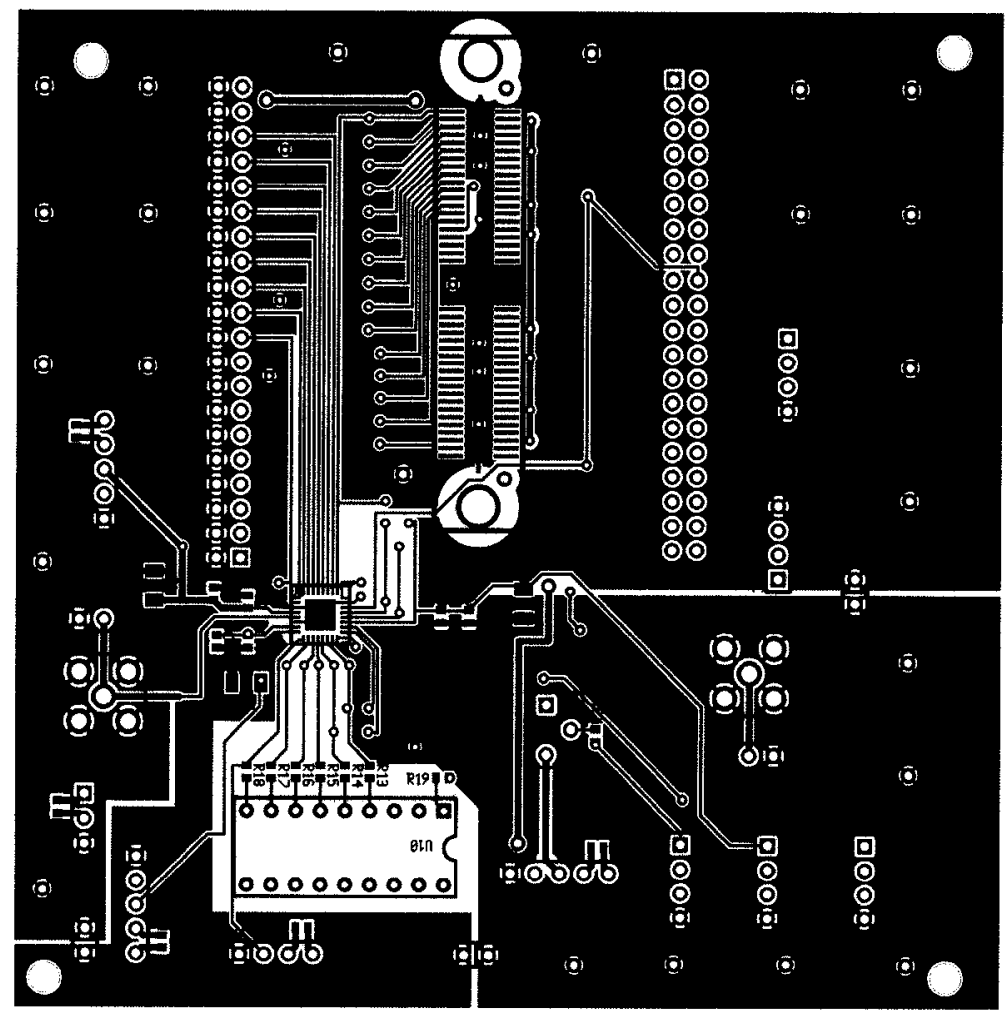

(a) Layout of the front side of the PCB.

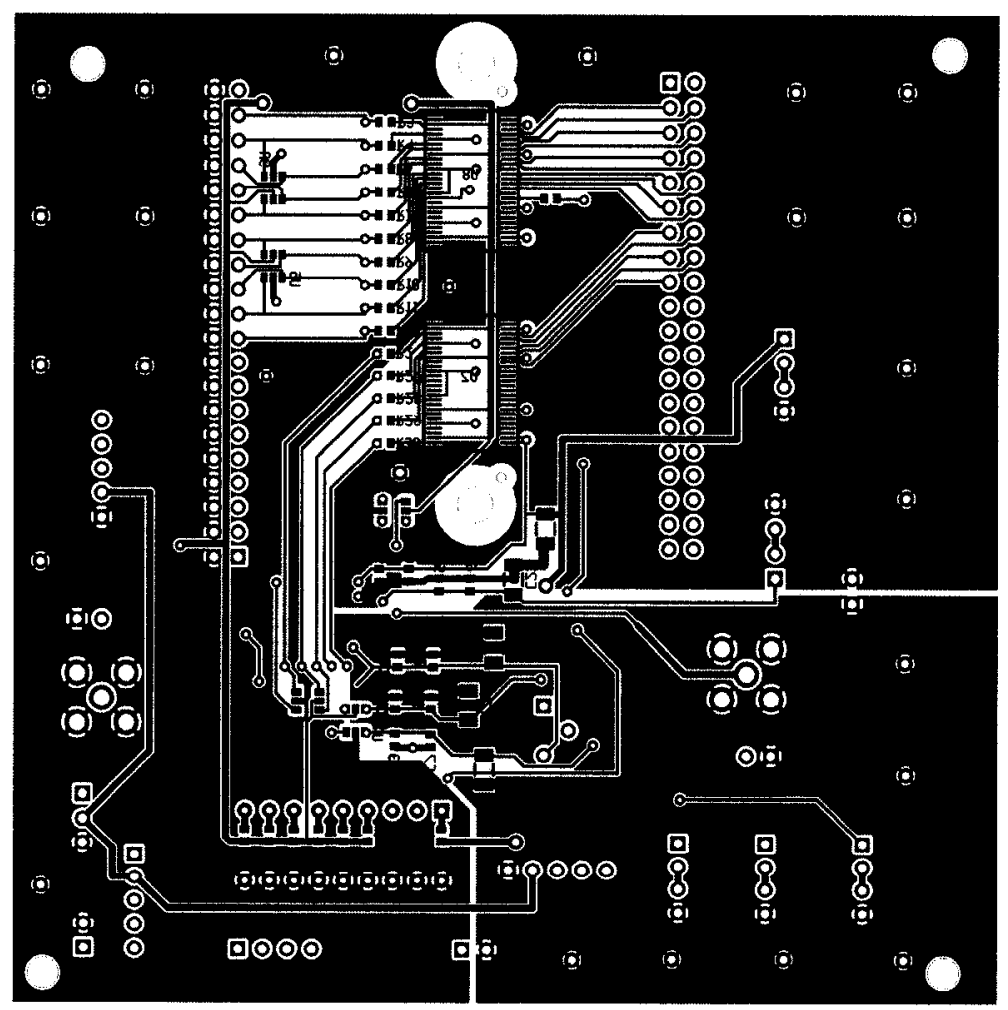

(b) Layout of the back side of the PCB.

Figure 7.7: Layout of the designed PCB. 


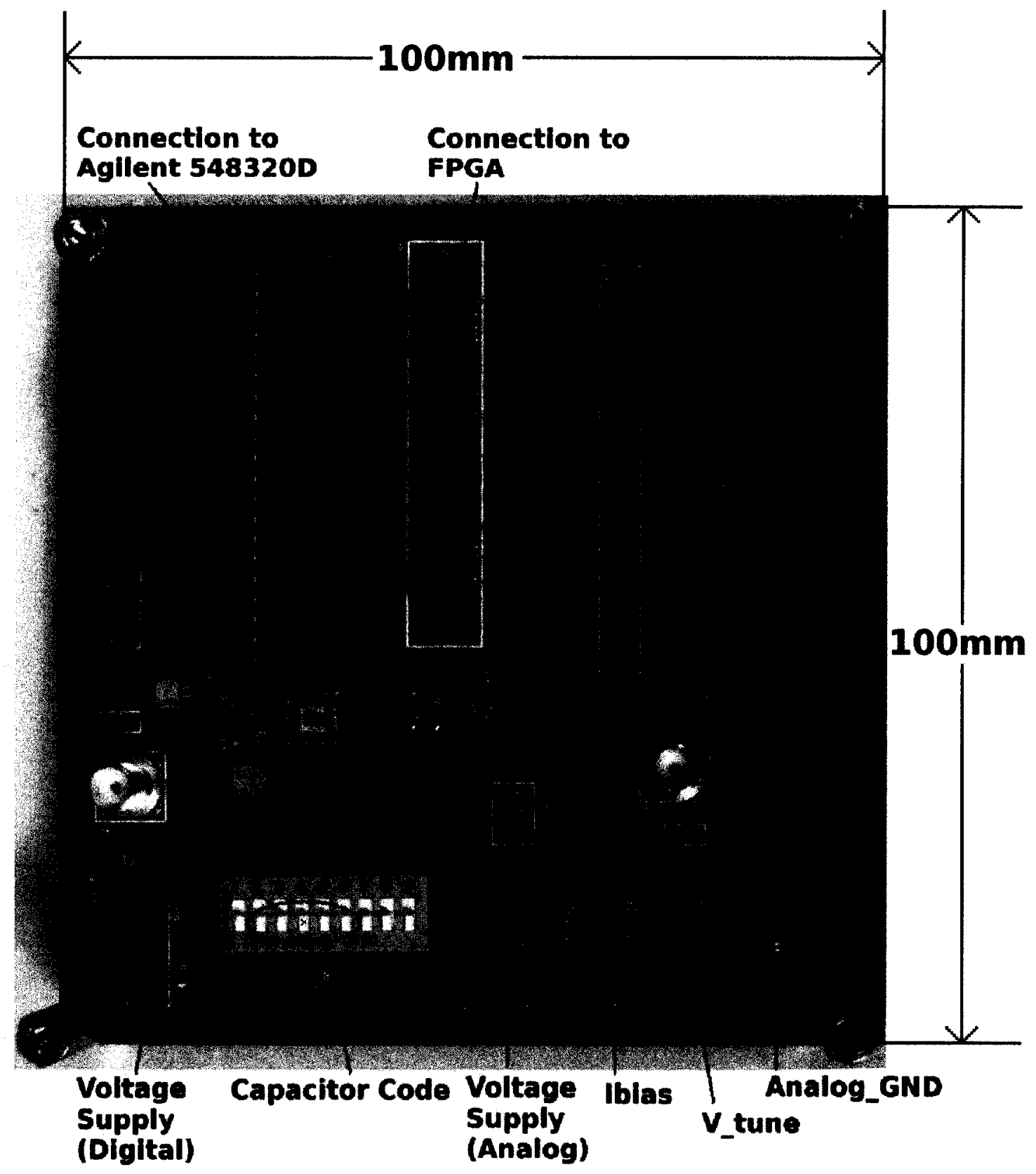

Figure 7.8: Labeled photograph of the PCB. 
from $\frac{1}{2} V_{D D}$ to $V_{D D}$ to the $\mathrm{ADC}$, at a frequency below the Nyquist frequency. The DFT of the digitized output was taken and the SFDR, SNR, SINAD, and ENOB were calculated from the DFT. This process was repeated for a range of input frequencies. The resulting SFDR is shown in Figure 7.9, the SNR and SINAD are shown in Figure 7.10, and the ENOB is shown in Figure 7.11. A simulation equivalent to this measurement could not be performed due to time restrictions. Measuring the effects of varying input frequencies and voltage supplies requires more time than was available during the course of the research.

Figure 7.9 (a) shows the SFDR when a $0.4 \mathrm{~V}$ supply is used for the ADC, at a $10 \mathrm{kS} / \mathrm{s}$ sample rate, as the input is varied from $5 \mathrm{~Hz}$ to $1 \mathrm{kHz}$. SFDR is initially above $45 \mathrm{~dB}$; however, at $30 \mathrm{~Hz}$, the SFDR started to sharply drop to approximately $25 \mathrm{~dB}$ by the time the input frequency reached $1 \mathrm{kHz}$. Figure $7.10(\mathrm{a})$ shows the SNR and SINAD under the same conditions. As was suggested in [2], the SINAD tracks closely with the SNR. This is because, even though SINAD incorporates both THD and SNR with equal weighting, ADCs generally exhibit very low harmonic distortion that SNR never approaches. The SNR and SINAD were at approximately $32 \mathrm{~dB}$ while the input frequency was below $30 \mathrm{~Hz}$. Increasing the input frequency caused the SNR and SINAD to tail off, finishing at approximately $10 \mathrm{~dB}$ when the input was $1 \mathrm{kHz}$. As the input frequency was increased towards the Nyquist frequency, the SFDR decreased by approximately $25 \mathrm{~dB}$, while the SNR and SINAD were decreased by approximately 20dB. At higher input frequencies, the input changed more drastically during the delay between the sampling of the input signal and the storing of the signal for 
conversion. This contributes to the drop-off in SFDR, SNR, and SINAD as the input frequency is increased.

Similar behaviour was observed in Figure 7.9(b), where a $0.6 \mathrm{~V}$ supply was used, at a $25 \mathrm{kS} / \mathrm{s}$ sample rate, as the input was varied from $10 \mathrm{~Hz}$ to $5 \mathrm{kHz}$. The SFDR was above $50 \mathrm{~dB}$ until the input frequency reached $200 \mathrm{~Hz}$, when it started to tail off to approximately $25 \mathrm{~dB}$ while approaching $5 \mathrm{kHz}$. The SNR and SINAD, from Figure 7.10 (b), were at approximately $37 \mathrm{~dB}$ for input frequencies below $200 \mathrm{~Hz}$, while they tailed off to approximately $15 \mathrm{~dB}$ by the time the input frequency reached $5 \mathrm{kHz}$.

When a $0.8 \mathrm{~V}$ supply was applied, with a $50 \mathrm{kS} / \mathrm{s}$ sample rate, the input frequency was varied from $40 \mathrm{~Hz}$ to $10 \mathrm{kHz}$. When the input frequency was below $200 \mathrm{~Hz}$, the SFDR, from Figure 7.9(c), was approximately $62 \mathrm{~dB}$, while it dropped to approximately $46 \mathrm{~dB}$ as the input frequency increased from $200 \mathrm{~Hz}$ to $10 \mathrm{kHz}$. The SNR and SINAD, from Figure $7.10(\mathrm{c})$, remained approximately constant at $40 \mathrm{~dB}$. When a $1 \mathrm{~V}$ $V_{D D}$ was applied to the circuit, with a $100 \mathrm{ks} / \mathrm{s}$ sample rate, the input was varied from $40 \mathrm{~Hz}$ to $10 \mathrm{kHz}$. The result from Figure $7.9(\mathrm{~d})$ shows that throughout the entire range, the SFDR stayed just below 55dB. Similar behaviour was noticed in Figure $7.9(\mathrm{e})$, where a $1.2 \mathrm{~V} V_{d d}$ was applied and a sample rate of $500 \mathrm{kS} / \mathrm{s}$ was used, with input frequencies varying between $200 \mathrm{~Hz}$ and 10kHz. Figure $7.10(\mathrm{~d})$ and Figure $7.10(\mathrm{e})$ show that the SNR and SINAD also remained mainly constant at approximately $40 \mathrm{~dB}$ throughout the sampled input frequency range. The reason behind this constant SINAD and SNR was investigated and it was found that when the ADC was bypassed and the input from the Stanford Research Systems DS360 signal generator was connected directly to the Agilent Infiniium 548320D MSO, the resulting noise 
floor was at $41 \mathrm{~dB}$, limiting performance to 6.5 effective bits. In future measurements, specifically the capacitor variation in Section 7.3.1, a low-pass filter will be applied at the digital output to reduce the noise floor in order to examine the ADC operation based mainly on harmonic distortion at the output signal.

Figure 7.11 shows the measured ENOB, as the input frequency is varied. These plots are identical to the SINAD curves, since ENOB has a linear relationship with SINAD, as shown in Equation 7.1. When a $0.4 \mathrm{~V}$ supply voltage was applied, the ENOB was approximately 4.9 bits for input frequencies below $30 \mathrm{~Hz}$, dropping to $1.5 \mathrm{bits}$ when the input frequency reached $1 \mathrm{kHz}$. When a $0.6 \mathrm{~V}$ supply voltage was applied, the ENOB was approximately 5.9 bits for input frequencies below $200 \mathrm{~Hz}$, dropping to 2.5 bits when the input frequency reached $5 \mathrm{kHz}$. Similar to the SINAD measurements, the ENOB remained approximately constant, at 6.35 bits, as the input frequency increased for cases when $V_{d d}$ was set to $0.8 \mathrm{~V}, 1 \mathrm{~V}$, and $1.2 \mathrm{~V}$.

Examining Figure 7.9, Figure 7.10, and Figure 7.11, it is evident that the dynamic performance of the ADC was improved as the voltage supply increases. SFDR increased from $47 \mathrm{~dB}$ at $V_{d d}=0.4 V$, to $52 \mathrm{~dB}$ at $V_{d d}=0.6 \mathrm{~V}$, and to $62 \mathrm{~dB}$ at $V_{d d}=0.8 \mathrm{~V}$. Similarly the SNR and SINAD increased from $32 \mathrm{~dB}$ at $V_{d d}=0.4 V$, to $37 \mathrm{~dB}$ at $V_{d d}=0.6 \mathrm{~V}$, and to $40 \mathrm{~dB}$ at $V_{d d}=0.8 \mathrm{~V}, 1.0 \mathrm{~V}, 1.2 \mathrm{~V}$. The ENOB increased on par with increasing SINAD, from 4.9 bits at $V_{d d}=0.4 V$, to 5.9 bits at $V_{d d}=0.6 \mathrm{~V}$, and to 6.35 bits at $V_{d d}=0.8 \mathrm{~V}, 1.0 \mathrm{~V}, 1.2 \mathrm{~V}$. When a higher supply voltage was used, the LSB increased, relaxing the accuracy requirements for the comparator and capacitor array. This improved harmonic distortion and increased the SINAD of the ADC. There was also a higher linearity achieved in the analog switches as the supply voltage was 


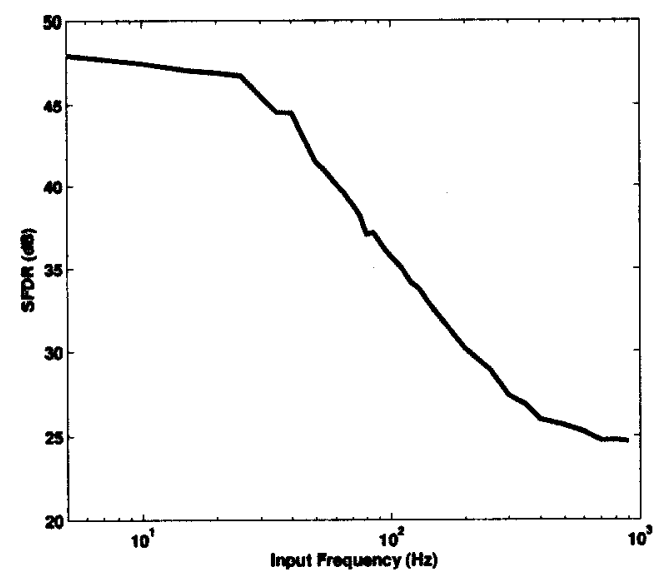

(a) $V_{D D}=0.4 \mathrm{~V}, \mathrm{SR}=10 \mathrm{kS} / \mathrm{s}$.

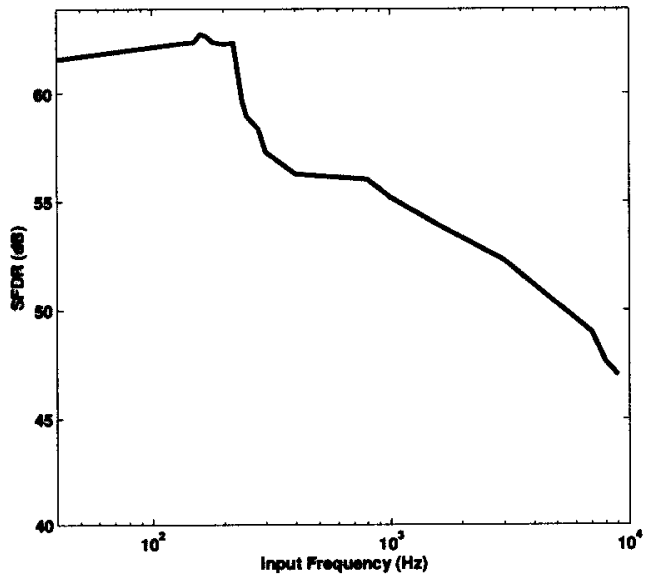

(c) $V_{D D}=0.8 \mathrm{~V}, \mathrm{SR}=50 \mathrm{kS} / \mathrm{s}$.

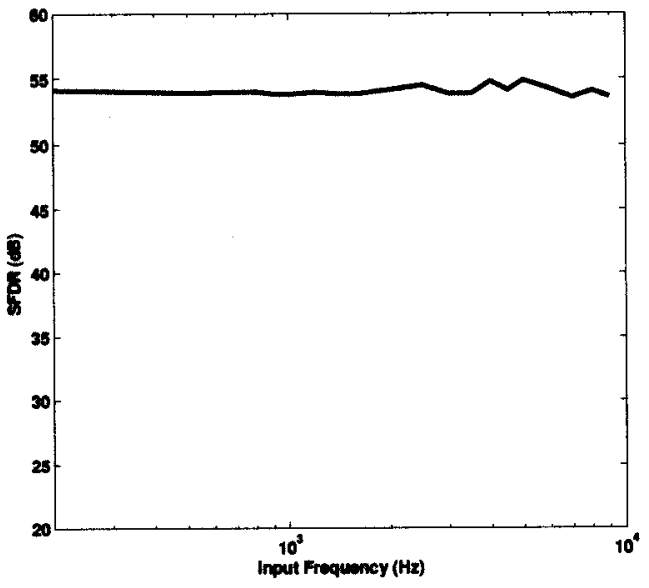

(e) $V_{D D}=1.2 \mathrm{~V}, \mathrm{SR}=500 \mathrm{kS} / \mathrm{s}$.

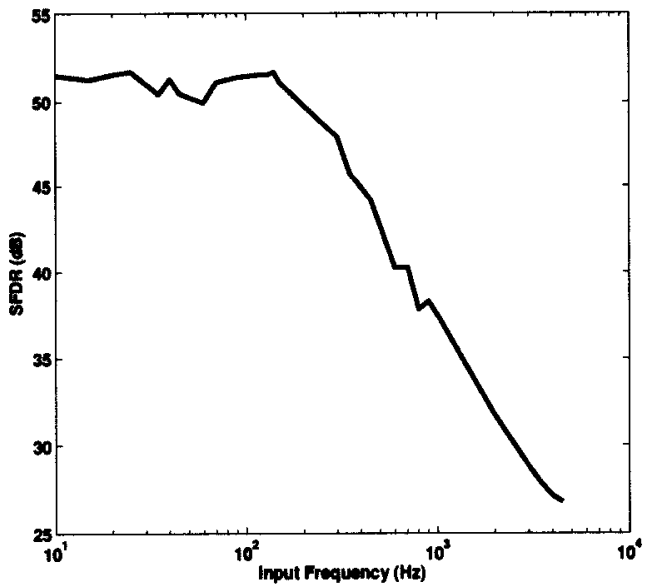

(b) $V_{D D}=0.6 \mathrm{~V}, \mathrm{SR}=25 \mathrm{kS} / \mathrm{s}$.

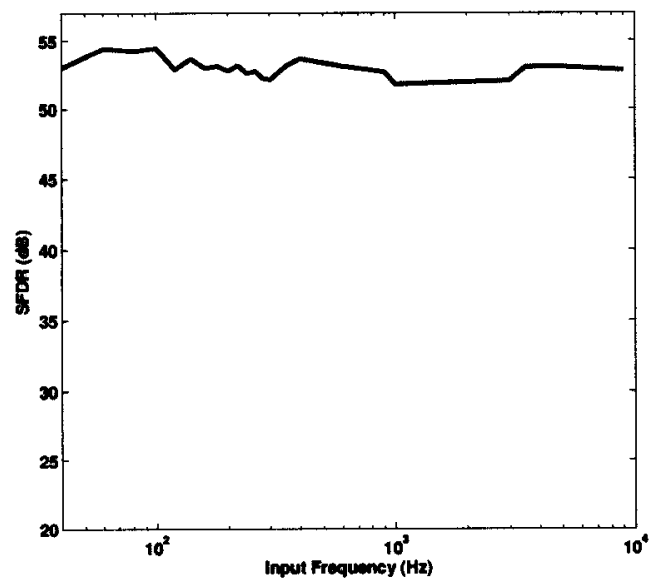

(d) $V_{D D}=1.0 \mathrm{~V}, \mathrm{SR}=100 \mathrm{kS} / \mathrm{s}$.

Figure 7.9: Measured SFDR vs. Input Frequency. 


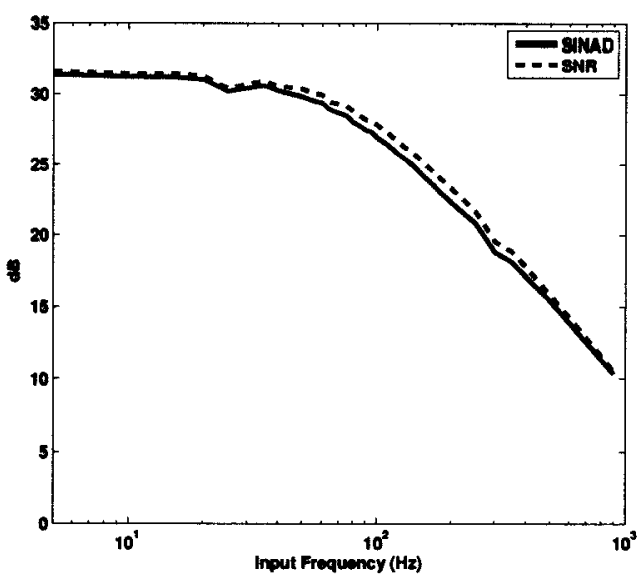

(a) $V_{D D}=0.4 \mathrm{~V}, \mathrm{SR}=10 \mathrm{kS} / \mathrm{s}$.

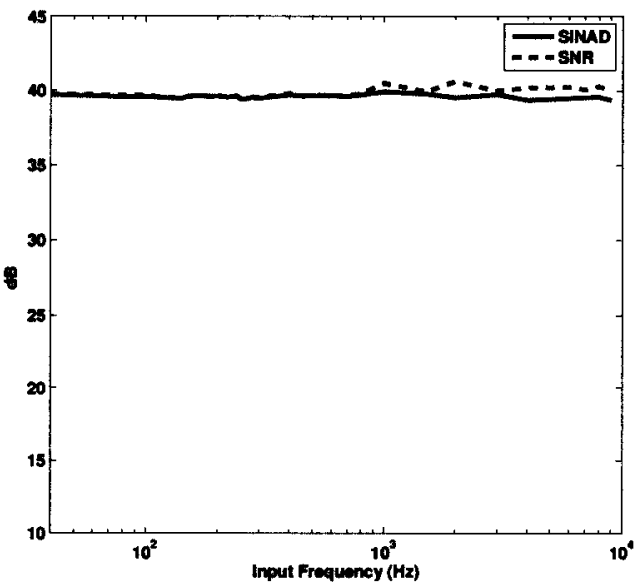

(c) $V_{D D}=0.8 \mathrm{~V}, \mathrm{SR}=50 \mathrm{kS} / \mathrm{s}$.

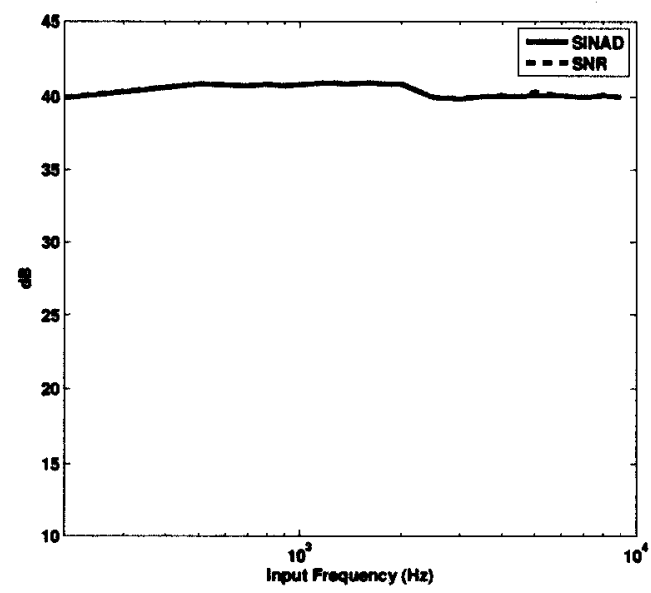

(e) $V_{D D}=1.2 \mathrm{~V}, \mathrm{SR}=500 \mathrm{kS} / \mathrm{s}$.

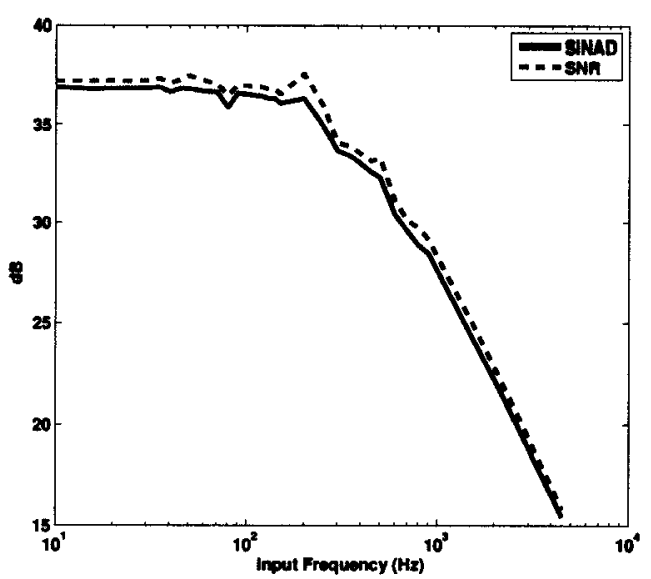

(b) $V_{D D}=0.6 \mathrm{~V}, \mathrm{SR}=25 \mathrm{kS} / \mathrm{s}$.

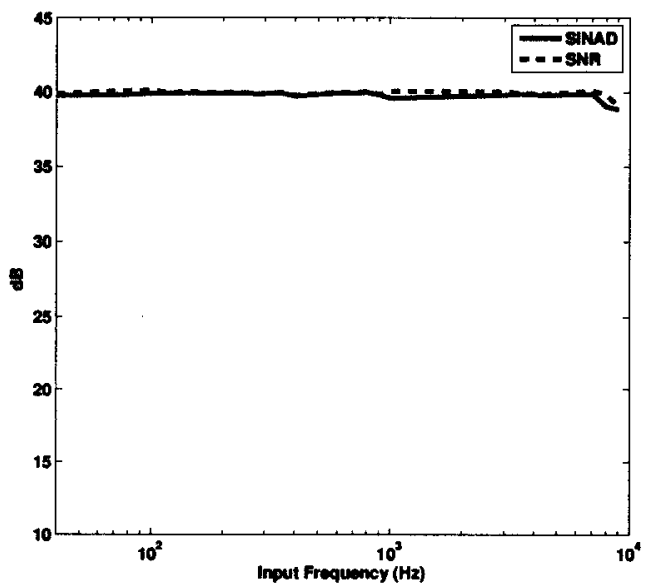

(d) $V_{D D}=1.0 \mathrm{~V}, \mathrm{SR}=100 \mathrm{kS} / \mathrm{s}$.

Figure 7.10: Measured SNR and SINAD vs. Input Frequency. 


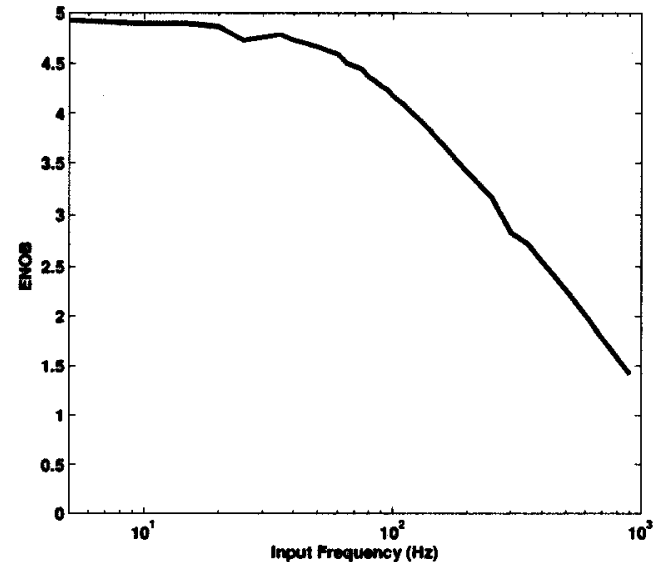

(a) $V_{D D}=0.4 \mathrm{~V}, \mathrm{SR}=10 \mathrm{kS} / \mathrm{s}$.

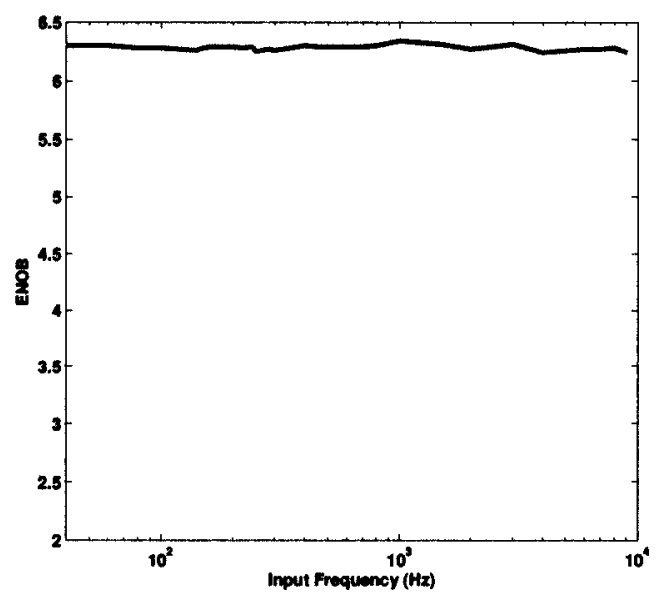

(c) $V_{D D}=0.8 \mathrm{~V}, \mathrm{SR}=50 \mathrm{kS} / \mathrm{s}$.

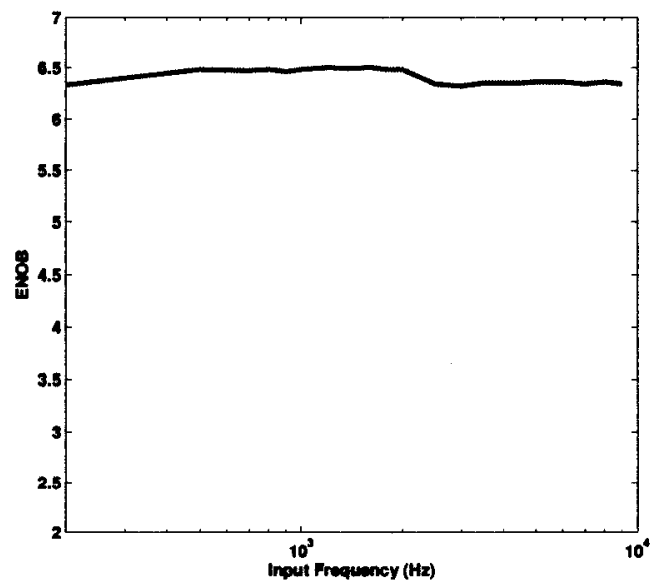

(e) $V_{D D}=1.2 \mathrm{~V}, \mathrm{SR}=500 \mathrm{kS} / \mathrm{s}$.

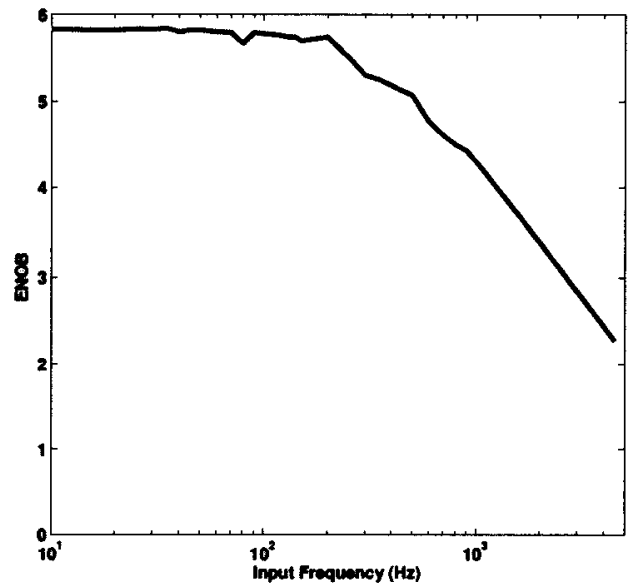

(b) $V_{D D}=0.6 \mathrm{~V}, \mathrm{SR}=25 \mathrm{kS} / \mathrm{s}$.

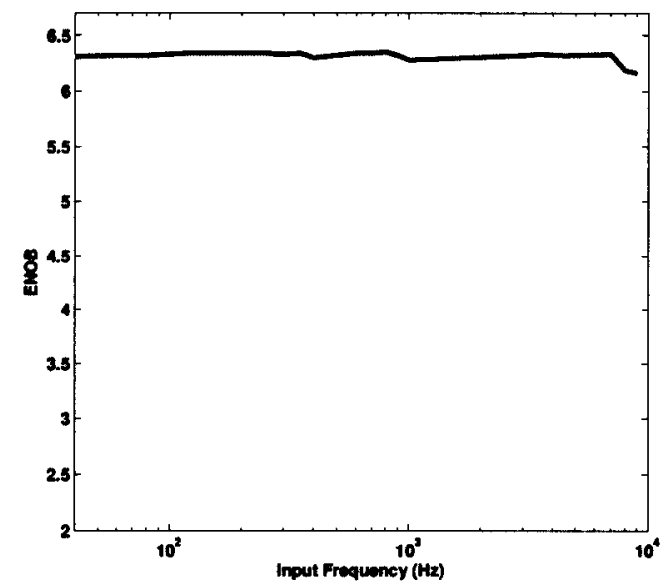

(d) $V_{D D}=1.0 \mathrm{~V}, \mathrm{SR}=100 \mathrm{kS} / \mathrm{s}$.

Figure 7.11: Measured ENOB vs. Input Frequency. 
increased, which increased the bandwidth of the ADC.

\subsubsection{Varying the Attenuation Capacitor}

Varying the digital bits that control the attenuation capacitor caused the capacitance to vary as shown in Figure 7.12. The attenuation capacitance does not vary linearly as the digital input code is varied. This is most evident in the transition between '01111' and '10000'. Using Equation 5.7, the capacitance that corresponds to the code ' 01111 ' is $619 \mathrm{fF}$, while the capacitance that corresponds to the code ' 10000 ' is $648 \mathrm{fF}$, even though the general trend is a decreasing capacitance as the input code increases.

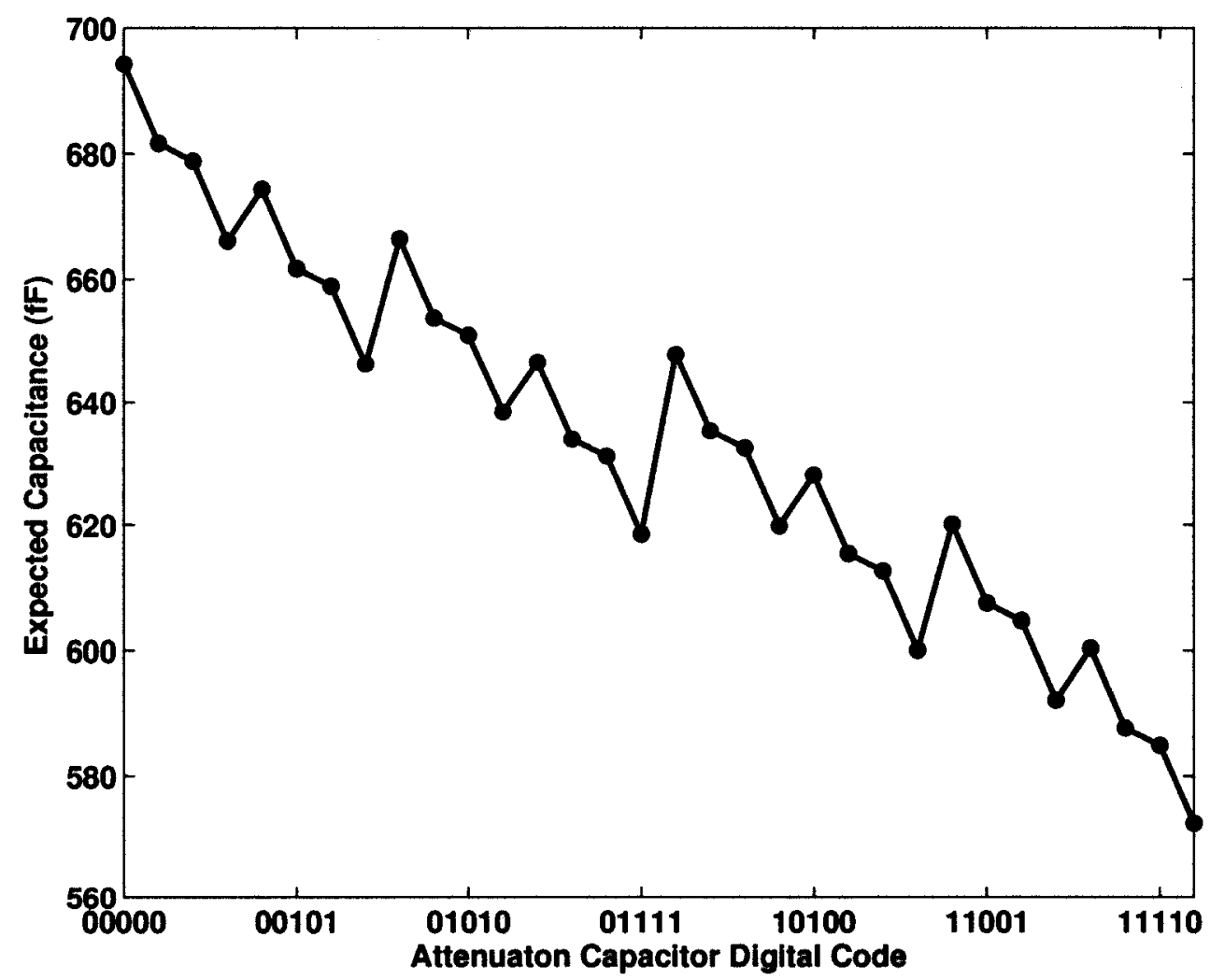

Figure 7.12: Effect of varying the attenuation capacitor code on the capacitance. 
For a supply voltage of $1.2 \mathrm{~V}$, the Xilinx Virtex II FPGA board was connected to the ADC's output bits and the attenuation capacitor code control bits. The capacitor control bits were assigned a register in the FPGA that is writable from MATLAB, allowing for the FPGA and MATLAB software to communicate with the chip. This setup allowed for a MATLAB code to modify the attenuation capacitance on the ADC, as well as to read the ADC's digital output bits. For each capacitor code, $64 \mathrm{kB}$ of output data was read into MATLAB using 16-bit words. The data was analyzed using an FFT function in MATLAB and SINAD was measured for each attenuation capacitor value. This type of analysis was performed only at a $V_{d d}$ of $1.2 \mathrm{~V}$ because the FPGA operates at $1.5 \mathrm{~V}$, and only $1.2 \mathrm{~V}$ operation from the $\mathrm{ADC}$ ensures that the FPGA will recognize a bit-wise HIGH value.

The dynamic performance of the ADC was measured as the attenuation capacitor was varied, at supply voltages of $0.4 \mathrm{~V}, 0.6 \mathrm{~V}, 0.8 \mathrm{~V}, 1.0 \mathrm{~V}$, and $1.2 \mathrm{~V}$. Table 7.1 shows the ADC sample rate and input frequency for each $V_{d d}$ tested. This setup was used to obtain results shown in Figure 7.13. A simulation of this measurement could not be performed due to time restrictions, as even performing a single dynamic measurement took a very long time.

Table 7.1: ADC Setup when testing attenuation capacitor code variation.

\begin{tabular}{|c|c|c|}
\hline$V_{d d}(V)$ & Sample Rate $(\mathrm{kS} / \mathbf{s})$ & Input Frequency $(\mathbf{H z})$ \\
\hline 0.4 & 10 & 50 \\
\hline 0.6 & 25 & 110 \\
\hline 0.8 & 50 & 170 \\
\hline 1.0 & 100 & 260 \\
\hline 1.2 & 500 & 1000 \\
\hline
\end{tabular}

Figure 7.13 shows the dynamic performance of the ADC when the attenuation 
capacitor was varied from $694 \mathrm{fF}$ to $572 \mathrm{fF}$; the full Nyquist range of frequencies were included in the measurements. The dynamic performance was measured with SINAD and ENOB in Figure 7.13(a) and with ENOB in Figure 7.13(b). There appears to be very little variance in SINAD as the attenuation capacitor was varied. With a $V_{d d}$ of $0.4 \mathrm{~V}$, the SINAD stayed approximately constant at $32.5 \mathrm{~dB}$, while the ENOB was approximately 5.1 bits. With a $V_{d d}$ of $0.6 \mathrm{~V}$, the SINAD stayed approximately constant at $39 \mathrm{~dB}$, while the ENOB was approximately 6.2 bits. With a $V_{d d}$ of $0.8 \mathrm{~V}$ and $1.0 \mathrm{~V}$, the SINAD stayed approximately constant at $39.9 \mathrm{~dB}$, while the ENOB was approximately 6.3 bits. With a $V_{d d}$ of $1.2 \mathrm{~V}$, the SINAD stayed approximately constant at $40 \mathrm{~dB}$, while the ENOB was approximately 6.35 bits. This constant behaviour at the output was expected, since the equipment introduced very high noise, causing it to be the limiting factor in the ADC measurement. The measured results for SINAD and ENOB in Figure 7.13 suggest that the ADC can likely perform better than the shown measured results at all available attenuation capacitor values if the equipment used had a better SNR.

In order to test the effectiveness of the attenuation capacitor tuning, the effects of the equipment had to be removed from the output. This was done by limiting the bandwidth of the output signal to reduce the effects of SNR and increase the effects of THD on SINAD with a low-pass filter. Once again, the dynamic performance of the ADC was tested while the attenuation capacitor was varied, at supply voltages of $0.4 \mathrm{~V}, 0.6 \mathrm{~V}, 0.8 \mathrm{~V}, 1.0 \mathrm{~V}$, and $1.2 \mathrm{~V}$. Table 7.2 shows the ADC sample rate, input frequency, and the bandwidth of the output signal for each $V_{d d}$ measured. This setup was used to obtain results shown in Figure 7.14. 


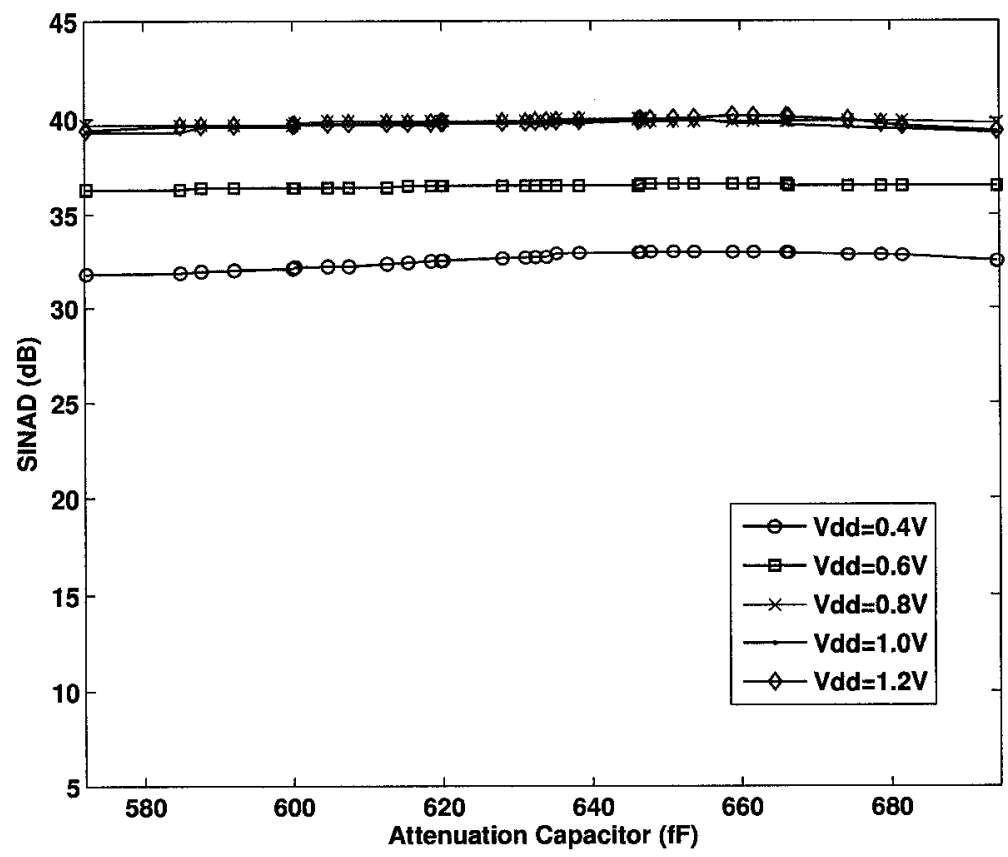

(a) Measured SINAD for the ADC as the capacitor code is varied.

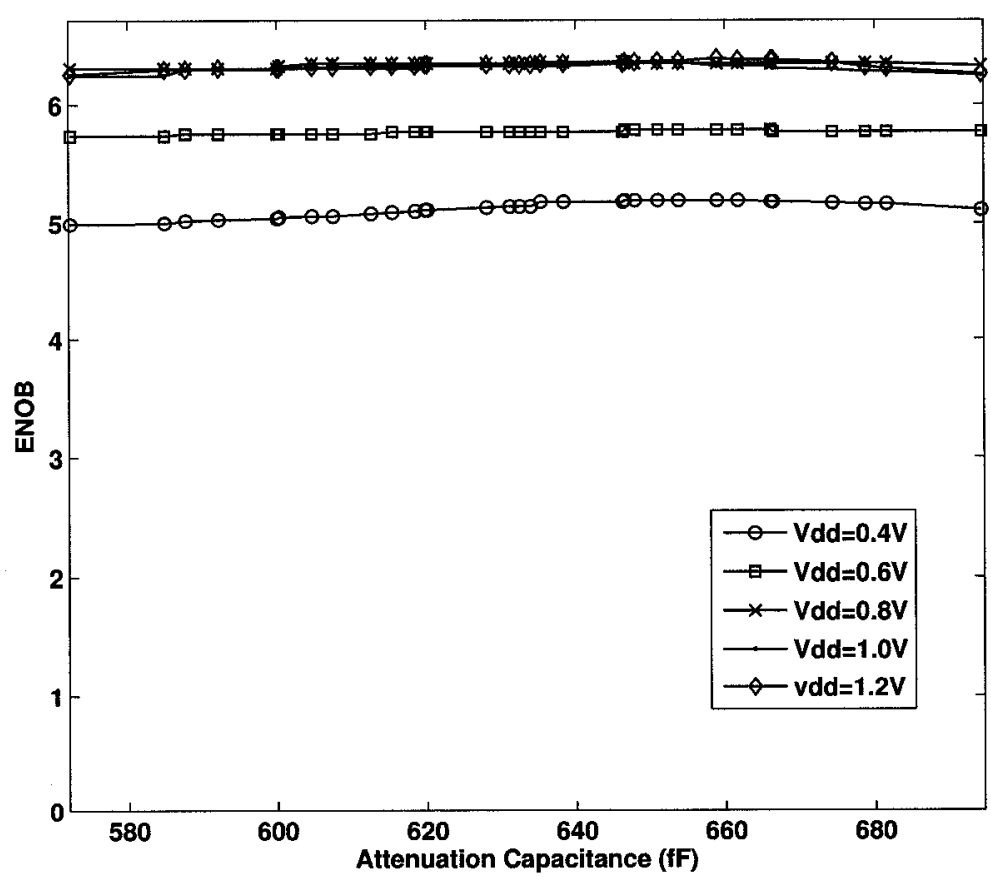

(b) Measured $\mathrm{ENOB}$ for the $\mathrm{ADC}$ as the capacitor code is varied.

Figure 7.13: Varying the Capacitor Code - Includes Full Nyquist Bandwidth. 
Table 7.2: ADC Setup when testing attenuation capacitor code variation.

\begin{tabular}{|c|c|c|r|}
\hline$V_{d d}$ & Sample Rate & Input Frequency & Bandwidth \\
\hline \hline $0.4 \mathrm{~V}$ & $10 \mathrm{kS} / \mathrm{s}$ & $50 \mathrm{~Hz}$ & $250 \mathrm{~Hz}$ \\
\hline $0.6 \mathrm{~V}$ & $25 \mathrm{kS} / \mathrm{s}$ & $110 \mathrm{~Hz}$ & $1000 \mathrm{~Hz}$ \\
\hline $0.8 \mathrm{~V}$ & $50 \mathrm{kS} / \mathrm{s}$ & $170 \mathrm{~Hz}$ & $1500 \mathrm{~Hz}$ \\
\hline $1.0 \mathrm{~V}$ & $100 \mathrm{kS} / \mathrm{s}$ & $260 \mathrm{~Hz}$ & $2000 \mathrm{~Hz}$ \\
\hline $1.2 \mathrm{~V}$ & $500 \mathrm{kS} / \mathrm{s}$ & $1000 \mathrm{~Hz}$ & $5000 \mathrm{~Hz}$ \\
\hline
\end{tabular}

Figure 7.14 shows the dynamic performance of the ADC when the attenuation capacitor code is varied from $694 \mathrm{fF}$ to $572 \mathrm{fF}$; the bandwidths used for the measurements are shown in Table 7.2. The dynamic performance is measured with SINAD in Figure 7.14(a) and ENOB in Figure 7.14(b).

With a $V_{d d}$ of $0.4 \mathrm{~V}$, the SINAD varied from a minimum of $40.7 \mathrm{~dB}$ to a maximum of $41.9 \mathrm{~dB}$, while the ENOB varied from 6.47 bits to 6.67 bits. The maximum SINAD and ENOB occurred when the digital input code for the attenuation capacitor was '01000', corresponding to a capacitance of $666.4 \mathrm{fF}$. This suggests that processing errors shifted the value of the capacitance in the capacitor array, causing the optimal attenuation capacitance to shift from $647 \mathrm{fF}$ to $666 \mathrm{fF}$.

With a $V_{d d}$ of $0.6 \mathrm{~V}$, the SINAD varied from a minimum of $48.7 \mathrm{~dB}$ to a maximum of 49.2dB, while the ENOB varied from 7.8 bits to 7.9 bits. The maximum SINAD and ENOB occurred when the digital input code for the attenuation capacitor was '01000', corresponding to a capacitance of $666.4 \mathrm{fF}$, similar to the case where $V_{d d}=0.4 \mathrm{~V}$.

With a $V_{d d}$ of $0.8 \mathrm{~V}$, the SINAD varied from a minimum of $51.3 \mathrm{~dB}$ to a maximum of $53.2 \mathrm{~dB}$, while the ENOB varied from 8.2 bits to 8.5 bits. The maximum SINAD and ENOB occurred when the digital input code for the attenuation capacitor was '01100', corresponding to a capacitance of $647 \mathrm{fF}$. This was the designed ideal value 
of the attenuation capacitance, suggesting that the tuning range of the attenuation capacitor was properly designed to include the effects of the parasitic capacitances.

With a $V_{d d}$ of $1.0 \mathrm{~V}$, the SINAD varied from a minimum of $53.9 \mathrm{~dB}$ to a maximum of $57.5 \mathrm{~dB}$, while the ENOB varied from 8.7 bits to 9.3 bits. The maximum SINAD and ENOB occurred when the digital input code for the attenuation capacitor was '01010', corresponding to a capacitance of $651 \mathrm{fF}$. Therefore, processing errors shifted the value of the capacitance in the capacitor array, causing the optimal attenuation capacitance to shift from $647 \mathrm{fF}$ to $651 \mathrm{fF}$ when a $1 \mathrm{~V}$ supply was used.

With a $V_{d d}$ of $1.2 \mathrm{~V}$, the SINAD varied from a minimum of $54.4 \mathrm{~dB}$ to a maximum of $60.7 \mathrm{~dB}$, while the ENOB varied from 8.7 bits to 9.8 bits. The maximum SINAD and ENOB occurred when the digital input code for the attenuation capacitor was '01100', corresponding to a capacitance of $634 \mathrm{fF}$. Therefore, processing errors shifted the value of the capacitance in the capacitor array, causing the optimal attenuation capacitance to shift from $647 \mathrm{fF}$ to $634 \mathrm{fF}$ when a $1.2 \mathrm{~V}$ supply is used.

It is evident that the optimal attenuation capacitor varies with supply voltage. The difference noted in the attenuation capacitor may be due to the parasitic capacitance on the switches used to control the digital input bits. Varying the voltage supply for the switches can vary the parasitic capacitance for the transistors used to design the analog switch. The resulting parasitic capacitance takes away from the required attenuation capacitance, therefore lowering the attenuation capacitor value needed for optimal dynamic operation. Figure 7.14 shows that the optimal capacitor code is found near the middle of the available tuning range. Varying the voltage supply from $0.4 \mathrm{~V}$ to $1.2 \mathrm{~V}$ has varied the optimal capacitor value from $666 \mathrm{fF}$ to $634 \mathrm{fF}$, 
while the tuning range is from $572 \mathrm{fF}$ to $694 \mathrm{fF}$. This is expected, as the design was optimized with the parasitic capacitances included in the simulations.

\subsection{Power Dissipation}

Figure 7.15 shows the power dissipation of the full ADC for varying sample rates and for supply voltages of $0.6 \mathrm{~V}, 0.8 \mathrm{~V}, 1.0 \mathrm{~V}$, and $1.2 \mathrm{~V}$. The power is divided between the power required by the digital part of the circuit and the analog part. It is evident that the digital components require much less power than the analog components, such as various switches and the comparator. At standby, the analog components dissipated power, while the digital power was negligible. As slow sample rates were applied, the digital ADC components amounted to approximately $3 \%$ of the total power dissipation. As the sample rate was increased, the digital power increased almost linearly. This was expected since the digital power was the average dynamic power of the SAR circuit, which increases with increasing frequency of operation. The analog power also increased due to the increased frequency of charging and discharging the switches and capacitors present in the analog circuit. The increasing sample rate resulted in the digital components having an increasing influence on the total ADC power. For example, when a $V_{D D}$ of $1 \mathrm{~V}$ was applied and the sample rate was varied from $20 \mathrm{kS} / \mathrm{s}$ to $1 \mathrm{MS} / \mathrm{s}$, the digital power varied from $3 \%$ to $37 \%$ of the total ADC power, respectively.

The measured power dissipation for an $\mathrm{ADC}$ with a $V_{D D}$ of $0.4 \mathrm{~V}$, sampled at $10 \mathrm{kS} / \mathrm{s}$ was $7.6 \mathrm{nW}$ for the digital power and $280 \mathrm{nW}$ for the analog power, resulting in a total power dissipation of $288 \mathrm{nW}$. When $V_{d d}$ was increased to $0.6 \mathrm{~V}$, the sample 


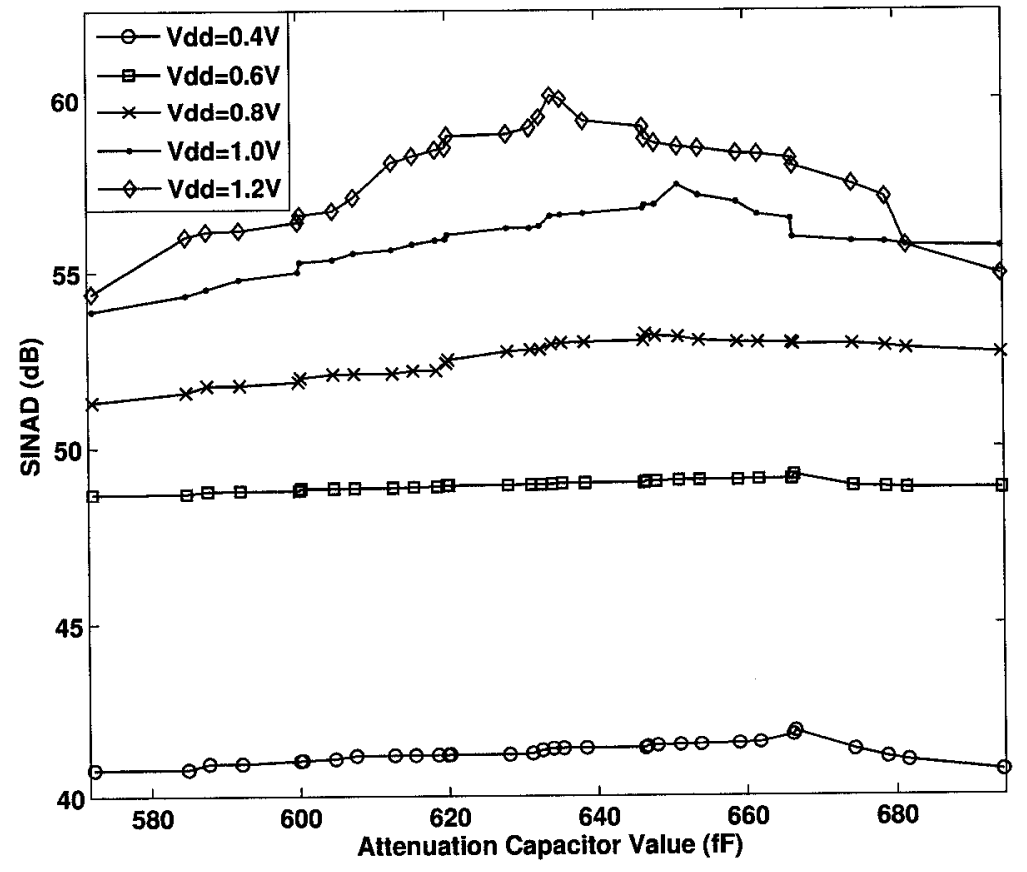

(a) Measured SINAD for the ADC as the capacitor code is varied.

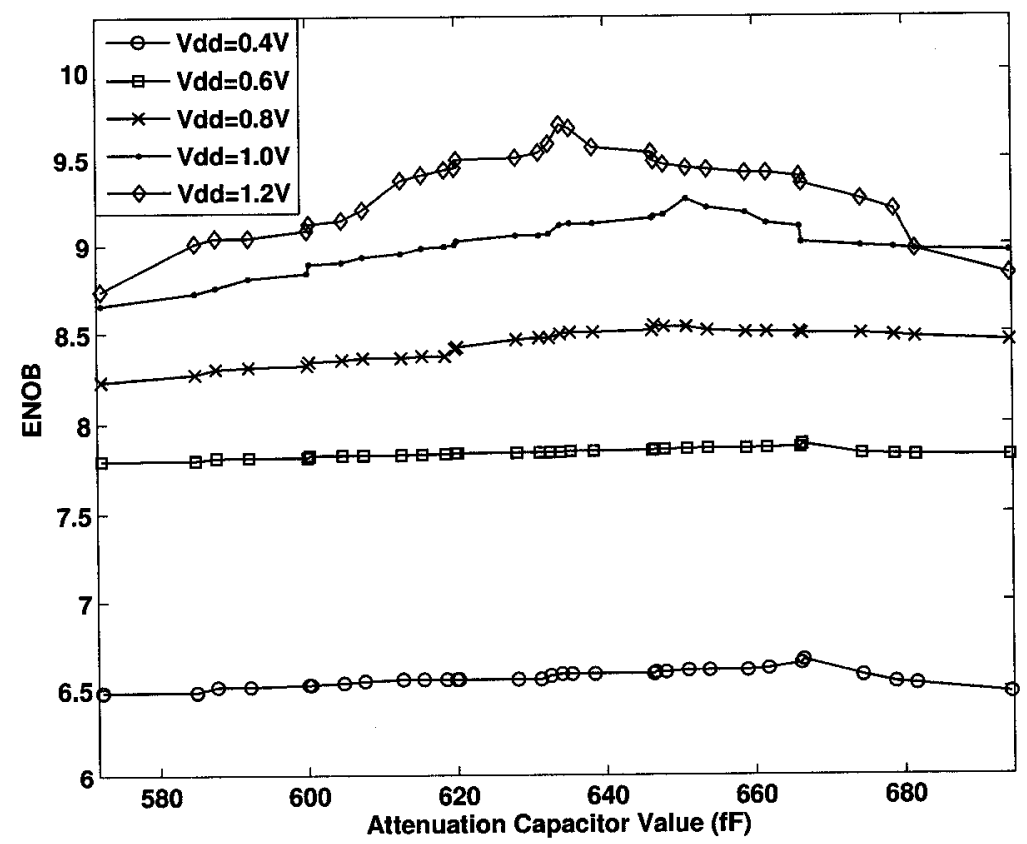

(b) Measured ENOB for the ADC as the capacitor code is varied.

Figure 7.14: Varying the Capacitor Code. 


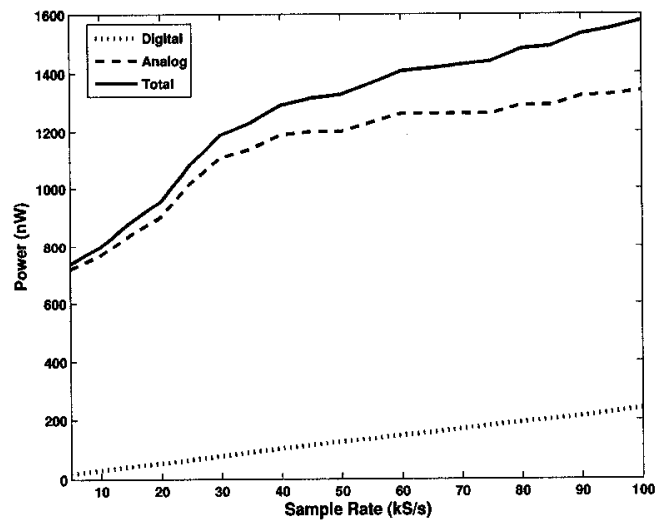

(a) $V_{D D}=0.6 \mathrm{~V}$.

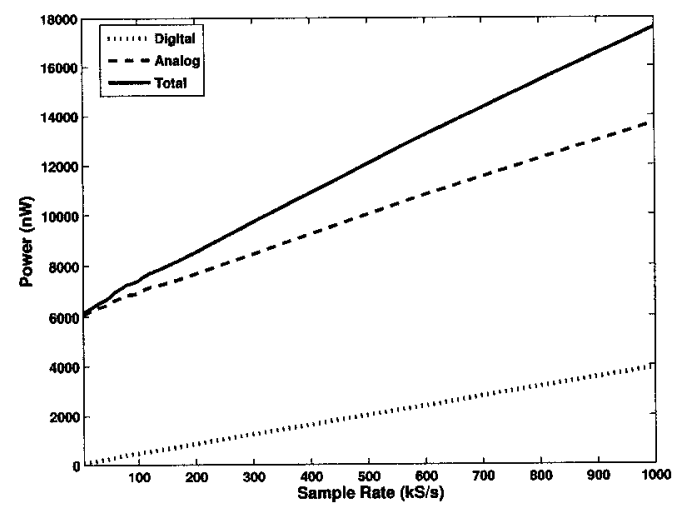

(c) $V_{D D}=1.0 \mathrm{~V}$.

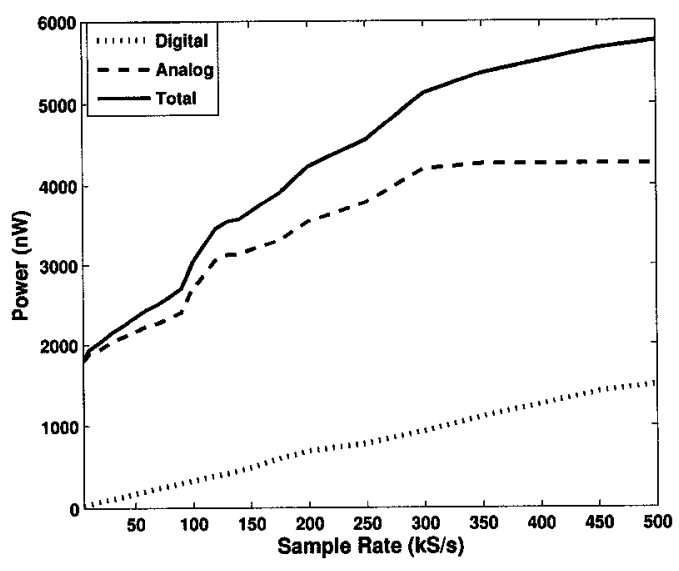

(b) $V_{D D}=0.8 \mathrm{~V}$.

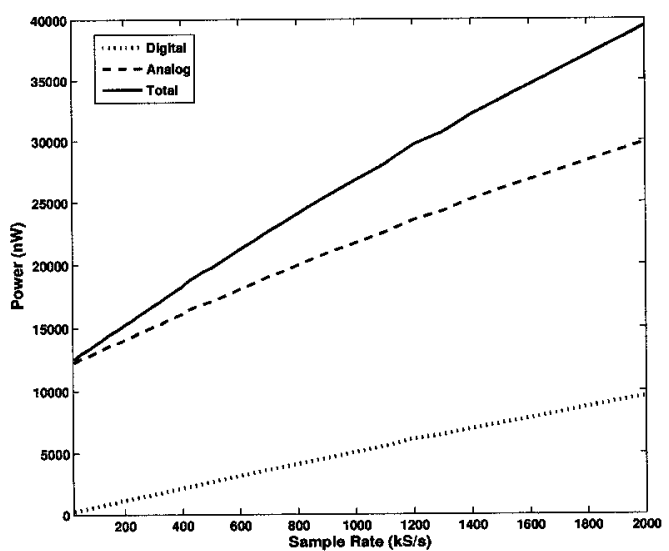

(d) $V_{D D}=1.2 \mathrm{~V}$.

Figure 7.15: Measured power dissipation of the full ADC vs. Sample rate. 
rate was increased to $25 \mathrm{kS} / \mathrm{s}$. The resulting total power dissipation was $1.09 \mu \mathrm{W}$. Increasing the voltage supply, $V_{D D}$, to $0.8 \mathrm{~V}$, sampled at $50 \mathrm{kS} / \mathrm{s}$, resulted in the ADC dissipating $2.34 \mu \mathrm{W}$. The measured power dissipation for the ADC was $7.41 \mu \mathrm{W}$ for a $V_{D D}$ of $1.0 \mathrm{~V}$, sampled at $100 \mathrm{kS} / \mathrm{s}$, while the $\mathrm{ADC}$ dissipated $19.71 \mu W$ at a $V_{D D}$ of $1.2 \mathrm{~V}$, sampled at $500 \mathrm{kS} / \mathrm{s}$.

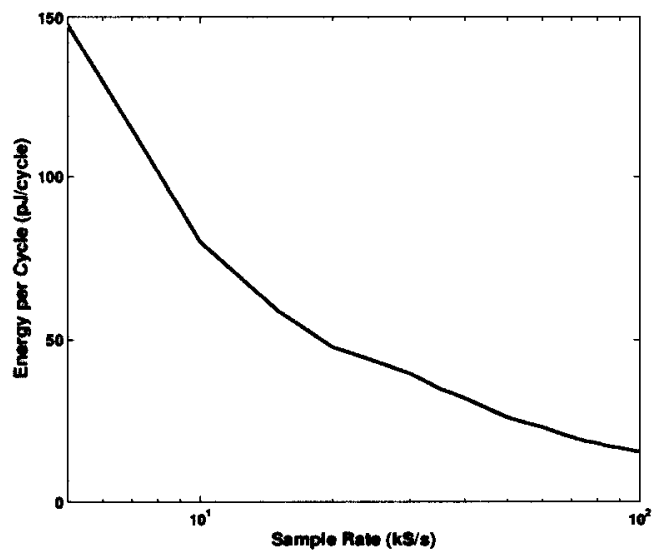

(a) $V_{D D}=0.6 \mathrm{~V}$.

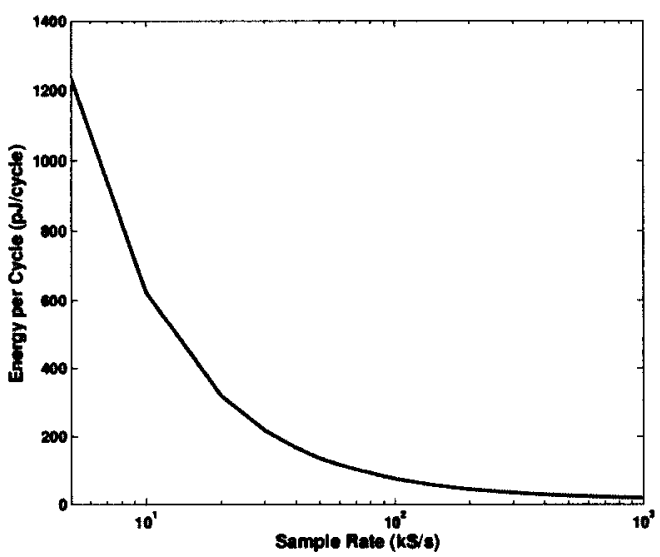

(c) $V_{D D}=1.0 \mathrm{~V}$.

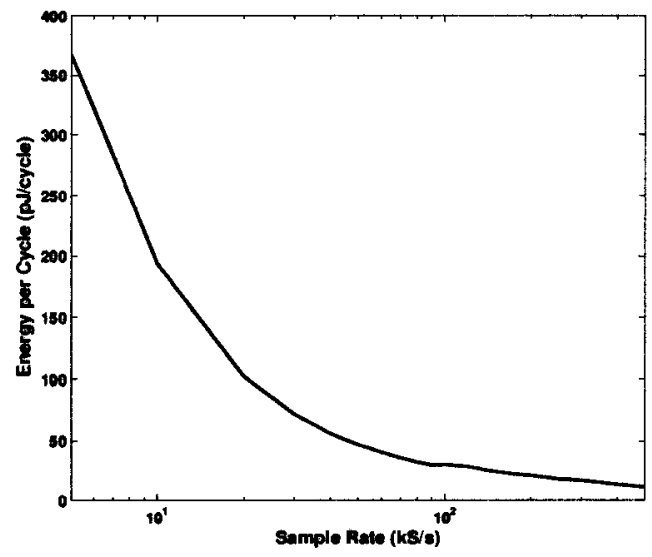

(b) $V_{D D}=0.8 \mathrm{~V}$.

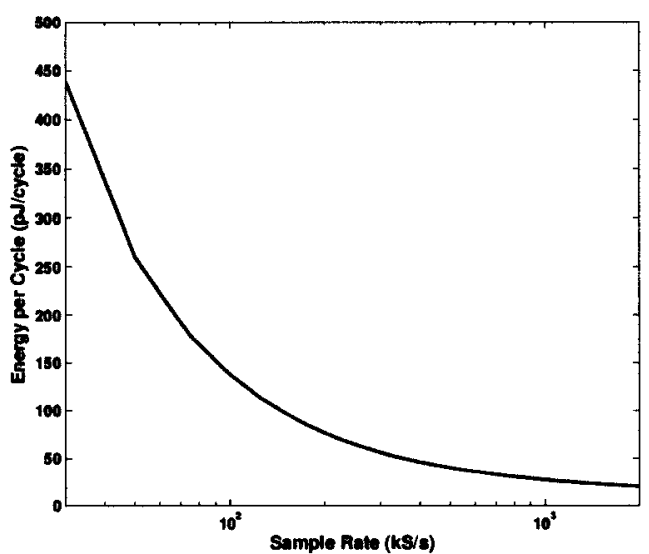

(d) $V_{D D}=1.2 \mathrm{~V}$.

Figure 7.16: Measured energy per cycle of the full ADC vs. Sample rate.

Figure 7.16 shows the energy per conversion cycle of the ADC as the sample rate was varied for supply voltages of $0.6 \mathrm{~V}, 0.8 \mathrm{~V}, 1.0 \mathrm{~V}$, and $1.2 \mathrm{~V}$. The curves follow the same pattern for each of the measured voltages. At low sample rates, the energy 
Table 7.3: Summary of measured power consumption vs. $V_{D D}$.

\begin{tabular}{|c|c|c|c|}
\hline$V_{D D}(V)$ & $\begin{array}{c}\text { Sample } \\
\text { Rate }(\mathrm{kS} / \mathrm{s})\end{array}$ & $\begin{array}{c}\text { Power Consumption } \\
(\mu W)\end{array}$ & $\begin{array}{c}\text { Energy per Cycle } \\
(\mathbf{p J} / \text { cycle })\end{array}$ \\
\hline 0.4 & 10 & 0.29 & 28.8 \\
\hline 0.6 & 25 & 1.09 & 43.4 \\
\hline 0.8 & 50 & 2.34 & 46.7 \\
\hline 1.0 & 100 & 7.41 & 74.1 \\
\hline 1.2 & 500 & 19.71 & 39.4 \\
\hline
\end{tabular}

required per conversion was high, and it decreases as the sample rate was increased. At lower sample rates, the constant $I_{\text {bias }}$ caused the bandwidth of the comparator to be higher than necessary, causing a high energy per conversion cycle. At higher sample rates, the dynamic digital power became more significant. The best case energy per conversion cycle occurred while the ADC is fully submerged in the subthreshold region, with a $V_{D D}$ of $0.4 \mathrm{~V}$, resulting in an energy per conversion cycle of $28.8 \mathrm{pJ} / \mathrm{cycle}$. However, this also results in the least accurate dynamic performance for the ADC and the slowest sample rate, since increasing the supply voltage to the ADC increases its dynamic performance. The energy per conversion cycle for the remaining supply voltages is shown in Figure 7.16 and summarized in Table 7.3.

Table 7.4 shows the power dissipation for the ADC as the supply voltage, $V_{d d}$ is varied, while the sample rate remains at $25 \mathrm{kS} / \mathrm{s}$. A plot of the digital power, as the supply voltage is varied, is shown in Figure 7.17. The graph suggests that the digital power increases almost linearly with increasing $V_{d d}$ while submerged in subthreshold operation; however, when the circuit enters the saturation region, the power dissipation increases significantly. It is expected that the dynamic power dissipation in saturation will increase quadratically, since the dynamic power is proportional to 
Table 7.4: Summary of power dissipation vs. $V_{d d}$ when the sample rate is $25 \mathrm{kS} / \mathrm{s}$.

\begin{tabular}{|c|c|c|c|c|}
\hline$V_{d d}$ & Digital Power & Analog Power & Total Power & \% Digital \\
\hline \hline $0.6 \mathrm{~V}$ & $66 \mathrm{nW}$ & $1020 \mathrm{nW}$ & $1086 \mathrm{nW}$ & $6.08 \%$ \\
\hline $0.8 \mathrm{~V}$ & $100 \mathrm{nW}$ & $1995 \mathrm{nW}$ & $2095 \mathrm{nW}$ & $4.77 \%$ \\
\hline $1.0 \mathrm{~V}$ & $147 \mathrm{nW}$ & $6288 \mathrm{nW}$ & $6435 \mathrm{nW}$ & $2.28 \%$ \\
\hline $1.2 \mathrm{~V}$ & $258 \mathrm{nW}$ & $12300 \mathrm{nW}$ & $12558 \mathrm{nW}$ & $2.05 \%$ \\
\hline
\end{tabular}

$V_{d d}^{2}[24]$. Table 7.4 also shows that the digital power is a more significant component of the total power dissipation in subthreshold, at $6 \%$, while in saturation, the digital power reduces to only $2 \%$ of the total power dissipation for the ADC.

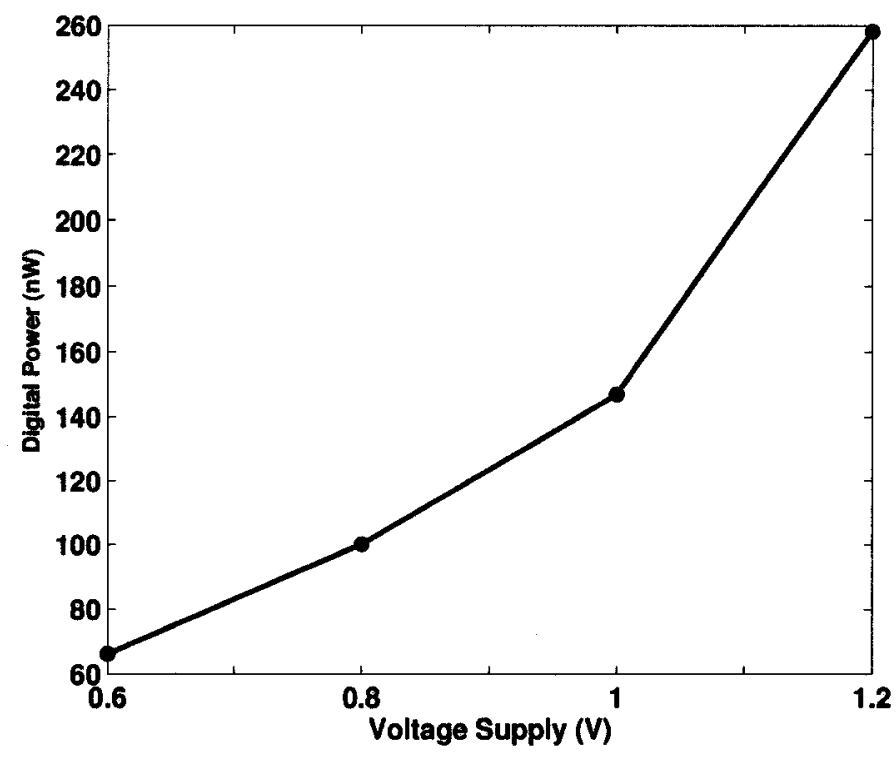

Figure 7.17: Effect of varying the voltage supply on the digital power, with sample rate $=25 \mathrm{kS} / \mathrm{s}$.

\subsection{Summary of the Measured ADC Results}

The measured results for the presented ADC are summarized in Table 7.5. The input range of the ADC was half of the supply voltage, and it ranged from $\frac{1}{2} V_{d d}$ to $V_{d d}$.

The dynamic measurements were performed by varying the attenuation capacitor and noting the effects of the variation on the SINAD and the ENOB at voltage 
supplies of $0.4 \mathrm{~V}, 0.6 \mathrm{~V}, 0.8 \mathrm{~V}, 1.0 \mathrm{~V}$, and $1.2 \mathrm{~V}$. As the supply voltage for the ADC was increased, the linearity requirement for the ADC relaxed and improved dynamic performance was noted. For a supply voltage of $0.4 \mathrm{~V}$, the input range is only $0.2 \mathrm{~V}$, meaning that the LSB is $195.5 \mu \mathrm{V}$. As the power supply increased to $1.2 \mathrm{~V}$, the input range increased to $0.6 \mathrm{~V}$, and the LSB became $586.5 \mu \mathrm{V}$.

The best dynamic performance was noted when $V_{d d}$ was set to $1.2 \mathrm{~V}$ with a sample rate of $500 \mathrm{kS} / \mathrm{s}$. The input signal was set to $1 \mathrm{kHz}$ with a bandwidth of $5 \mathrm{kHz}$. The resulting measured SINAD was $60.7 \mathrm{~dB}$, corresponding to $9.8 \mathrm{ENOB}$, when the attenuation capacitor was set to $634 \mathrm{fF}$. The worst dynamic performance was noted when the ADC was fully in the subthreshold region of operation, at a $V_{d d}$ of $0.4 \mathrm{~V}$, sampled at $10 \mathrm{kHz}$. The input was a sinusoid at $30 \mathrm{~Hz}$, with a $250 \mathrm{~Hz}$ bandwidth, and the resulting SINAD was $41.9 \mathrm{~dB}$, corresponding to $6.67 \mathrm{ENOB}$, with an attenuation capacitance of $666.4 \mathrm{fF}$.

The power dissipation for the ADC increased exponentially with increasing voltage supply, as was noted during simulations. For a supply voltage of $0.6 \mathrm{~V}$, the power dissipation was found to be $1.09 \mu W$, while it grew to $19.71 \mu W$ at a $1.2 \mathrm{~V}$ supply. The digital components of the circuit have a negligible power dissipation at standby, while the analog components have some power dissipation due to the bias current for the comparator. This resulted in the analog power being much higher than the digital power at low frequencies (up to $97 \%$ ). However, increasing the frequency caused the dynamic power of the digital circuit to increase and to influence the overall power by up to $40 \%$. 


\subsection{Comparison to Published ADCs}

A survey of recent IEEE publications for low-power 10-bit ADCs is shown in Table 7.6. These published designs were compared to the presented SAR ADC design, operating at $0.6 \mathrm{~V}$ and at $1.2 \mathrm{~V}$. The most important measure of the ADC, when energy requirements are stringent, is the energy per cycle, or the total energy required to complete a full conversion cycle [13].

$$
\text { Energy } y_{\text {cycle }}=\frac{\text { Power }}{F_{S}}
$$

Another measure of energy efficiency is the energy per state measurement. This measurement assumes that increasing resolution by 1 bit increases the power cost by 4 times, mainly useful for $\Sigma \Delta$ modulators [32]. The equation for energy per state, from [18], is

$$
\text { Energy } y_{\text {state }}=\frac{\text { Energy } y_{\text {cycle }}}{2^{E N O B}}
$$

For successive approximation $\mathrm{ADCs}$, the cost of adding an extra bit of resolution requires adding an extra charge cycling phase. This suggests a linear relationship between increasing resolution and the resulting power dissipation [32]. For the SAR architecture, a measure of energy per bit might be more useful [32]. The equation for the energy per bit, from [18] is

$$
\text { Energy }_{b i t}=\frac{\text { Energy }_{\text {cycle }}}{E N O B}
$$

The published 10-bit ADCs that are the most energy efficient are [21], [22], [46], and [47], shown in Table 7.6. The presented design offers an improved energy efficiency when compared to these designs while operating at $0.6 \mathrm{~V}$ and $1.2 \mathrm{~V}$ supplies. The 
energy per cycle, when operating at a $V_{d d}$ of $0.6 \mathrm{~V}$ is $43.4 \mathrm{pJ} /$ cycle, while at a $V_{d d}$ of $1.2 \mathrm{~V}$, the energy per cycle is $39.4 \mathrm{pJ} /$ cycle. The design that comes closest to the energy efficiency presented here is [22], with a $43.8 \mathrm{pJ} /$ cycle energy efficiency. This design has the lowest power dissipation, at $140 \mathrm{nW}$, while operating at $1.2 \mathrm{~V}$, with a very low, $25 \mathrm{~Hz}$, bandwidth. The $\mathrm{ADC}$ is geared towards a specialized application, the EEG, which requires frequencies between $0.2 \mathrm{~Hz}$ and $25 \mathrm{~Hz}$ [22]. The energy saving was accomplished by introducing a quasi-floating-gate (QFG) transistor for the class AB amplifier used in the design. The QFG transistor achieves lowered static power consumption with a minimal increase in circuit complexity [22]. The design also did not use a standard CMOS process, but rather settled for an AMIS C5 0.5 $\mu \mathrm{m}$ process with Epi Wafers.

The presented design also offers the lowest energy per state, at $0.044 \mathrm{pJ} / \mathrm{state}$, when operating at $1.2 \mathrm{~V}$. The $\Sigma \Delta$ design from [22] is close, at $0.049 \mathrm{pJ} / \mathrm{state}$. The SAR design from [47] also has a low energy per bit ratio, at $0.24 \mathrm{pJ} /$ bit. The design presented in [47] implements a DAC using a C-2C capacitor array and an attenuation capacitor in order to achieve lowered power consumption. The lowest energy per bit is also achieved with the presented design, when the supply voltage is $1.2 \mathrm{~V}$. The energy per bit is $4.0 \mathrm{pJ} /$ bit when $V_{d d}$ is $1.2 \mathrm{~V}$, while it is $5.5 \mathrm{pJ} /$ bit when $V_{d d}$ is $0.6 \mathrm{~V}$.

The total power dissipation of the presented $\mathrm{ADC}$ at $0.6 \mathrm{~V}$ is $1.09 \mu \mathrm{W}$, while it is $19.71 \mu \mathrm{W}$ at $1.2 \mathrm{~V}$. However, since the sample rate used when operating at $0.6 \mathrm{~V}$ was $25 \mathrm{kHz}$, while it was $500 \mathrm{kHz}$ when operating at $1.2 \mathrm{~V}$, the $1.2 \mathrm{~V}$ operation has better energy efficiency. 
Table 7.5: Summary of the measured ADC Results.

\begin{tabular}{|c||c|c|c|c|c|c|}
\hline \multicolumn{1}{|c||}{$V_{d d}$} & $\mathbf{0 . 4 V}$ & $\mathbf{0 . 6 V}$ & $\mathbf{0 . 8 V}$ & $\mathbf{1 . 0 V}$ & $\mathbf{1 . 2 V}$ \\
\hline \multicolumn{5}{|c||}{ Technology } & \multicolumn{5}{|c|}{$0.13 \mu \mathrm{m}$ CMOS } \\
\hline Area & $0.2 \mathrm{~V}$ & $0.3 \mathrm{~V}$ & $0.4 \mathrm{~V}$ & $0.5 \mathrm{~V}$ & $0.6 \mathrm{~V}$ \\
\hline Input Range & $10 \mathrm{kS} / \mathrm{s}$ & $25 \mathrm{kS} / \mathrm{s}$ & $50 \mathrm{kS} / \mathrm{s}$ & $100 \mathrm{kS} / \mathrm{s}$ & $500 \mathrm{kS} / \mathrm{s}$ \\
\hline \hline Sample Rate & $7.6 \mathrm{nW}$ & $66 \mathrm{nW}$ & $177 \mathrm{nW}$ & $480 \mathrm{nW}$ & $2.67 \mu W$ \\
\hline Digital Power & $280 \mathrm{nW}$ & $1.02 \mu W$ & $2.16 \mu W$ & $6.93 \mu W$ & $17.04 \mu W$ \\
\hline Analog Power & $288 \mathrm{nW}$ & $1.09 \mu W$ & $2.34 \mu W$ & $7.41 \mu W$ & $19.71 \mu W$ \\
\hline \hline Total Power & $28.8 \mathrm{pJ}$ & $43.4 \mathrm{pJ}$ & $46.7 \mathrm{pJ}$ & $74.1 \mathrm{pJ}$ & $39.4 \mathrm{pJ}$ \\
\hline Energy per Conversion & $0.28 \mathrm{pJ}$ & $0.18 \mathrm{pJ}$ & $0.13 \mathrm{pJ}$ & $0.12 \mathrm{pJ}$ & $0.04 \mathrm{pJ}$ \\
\hline Energy per State & $4.3 \mathrm{pJ}$ & $5.5 \mathrm{pJ}$ & $5.5 \mathrm{pJ}$ & $8 \mathrm{pJ}$ & $4 \mathrm{pJ}$ \\
\hline Energy per Bit & $30 \mathrm{~Hz}$ & $110 \mathrm{~Hz}$ & $170 \mathrm{~Hz}$ & $260 \mathrm{~Hz}$ & $1000 \mathrm{~Hz}$ \\
\hline Bin & $250 \mathrm{~Hz}$ & $1 \mathrm{kHz}$ & $1.5 \mathrm{kHz}$ & $2 \mathrm{kHz}$ & $5 \mathrm{kHz}$ \\
\hline \hline Bttenuation Capacitor & $666.4 \mathrm{fF}$ & $666.4 \mathrm{fF}$ & $647 \mathrm{fF}$ & $651 \mathrm{fF}$ & $634 \mathrm{fF}$ \\
\hline SINAD & $41.9 \mathrm{~dB}$ & $49.2 \mathrm{~dB}$ & $53.2 \mathrm{~dB}$ & $57.5 \mathrm{~dB}$ & $60.7 \mathrm{~dB}$ \\
\hline ENOB & $6.67 \mathrm{bits}$ & $7.9 \mathrm{bits}$ & $8.5 \mathrm{bits}$ & $9.3 \mathrm{bits}$ & $9.8 \mathrm{bits}$ \\
\hline
\end{tabular}

Table 7.6: Comparison of the presented design with published 10-bit ADC designs.

\begin{tabular}{|c||c|c|c|c||c|c|}
\cline { 2 - 7 } \multicolumn{1}{c||}{} & {$[\mathbf{2 1}]$} & {$[\mathbf{2 2}]$} & {$[\mathbf{4 6}]$} & {$[\mathbf{4 7}]$} & $\begin{array}{c}\text { This } \\
\text { Work }\end{array}$ & $\begin{array}{c}\text { This } \\
\text { Work }\end{array}$ \\
\hline ADC & SAR & $\Sigma \Delta$ & $\Sigma \Delta$ & SAR & SAR & SAR \\
\hline Technology & $0.18 \mu \mathrm{m}$ & $0.5 \mu \mathrm{m}$ & $0.13 \mu \mathrm{m}$ & $0.18 \mu \mathrm{m}$ & $0.13 \mu \mathrm{m}$ & $0.13 \mu \mathrm{m}$ \\
\hline$V_{D D}(\mathbf{V})$ & 1 & 1.2 & 0.7 & 1.5 & 0.6 & 1.2 \\
\hline Speed (kS/s) & 40 & 3.2 & 10 & 137 & 25 & 500 \\
\hline ENOB & 9.4 & 9.8 & 7.9 & 8.65 & 7.9 & 9.8 \\
\hline Power $(\mu W)$ & 32.6 & 0.14 & 3 & 13.4 & 1.09 & 19.71 \\
\hline $\begin{array}{c}\text { Energy } \\
\text { (pJ/cycle) }\end{array}$ & 815 & 43.8 & 300 & 97 & $\mathbf{4 3 . 4}$ & $\mathbf{3 9 . 4}$ \\
\hline $\begin{array}{c}\text { Energy } \\
\text { (pJ/state) }\end{array}$ & 1.21 & 0.049 & 1.26 & 0.24 & $\mathbf{0 . 1 8}$ & $\mathbf{0 . 0 4 4}$ \\
\hline $\begin{array}{c}\text { Energy } \\
\text { (pJ/bit) }\end{array}$ & 86.7 & 4.7 & 40 & 11.2 & $\mathbf{5 . 5}$ & $\mathbf{4 . 0}$ \\
\hline
\end{tabular}

[22] fabricated using Epi Wafers in an AMIS C5 process. 


\section{Chapter 8}

\section{Conclusion}

\subsection{Contributions and Achievements}

To the author's knowledge, the presented ADC has the best energy consumption when compared to currently published 10-bit ADC designs. The low power dissipation is achieved by taking advantage of the subthreshold properties of a transistor. Another advantage of the presented design is that it is fully implemented in a standard CMOS process, with no need for specialized processes such as Silicon-germanium (SiGe), Silicon carbide ( $\mathrm{SiC}$ ), silicon-on-sapphire (SOS), and BiCMOS. The ADC is designed to allow for processing and device mismatch variations by tuning of the series attenuation capacitor, used to reduce the size of the capacitor array.

The presented design takes advantage of subthreshold properties of transistors in order to operate the ADC at low voltage supplies with low power dissipation. The full $\mathrm{ADC}$, operating from a $0.6 \mathrm{~V}$ supply was sampled at $25 \mathrm{kS} / \mathrm{s}$, resulting in a power dissipation of $1.09 \mu \mathrm{W}$. This results in an energy consumption that is among the lowest published 10-bit ADCs, with an energy per conversion cycle of $43.4 \mathrm{pJ} / \mathrm{cycle}$, an energy per state of $0.18 \mathrm{pJ} /$ state, and an energy per bit of $5.5 \mathrm{pJ} /$ bit. The energy consumption 
is very competitive with current published 10-bit ADCs and it can accommodate the needs of implantable biomedical applications and wireless sensor networks.

While operating at $1.2 \mathrm{~V}$, the design is no longer submerged in subthreshold, but still manages to have the lowest energy consumption among published 10-bit ADCs. Sampled at $500 \mathrm{kS} / \mathrm{s}$, the ADC dissipates $19.71 \mu \mathrm{W}$, resulting in an energy per conversion cycle of $39.4 \mathrm{pJ} /$ cycle, an energy per state of $0.044 \mathrm{pJ} / \mathrm{state}$, and an energy per bit of $4.0 \mathrm{pJ} /$ bit. The increased voltage supply allows the ADC to run at higher sample rates. A reasons behind the allowed increase in sample rate, and thereby the reduction in energy consumption was the use of an attenuation capacitor. The implementation of the attenuation capacitor ensured that the capacitor array had reasonably small capacitors that need to be charged and discharged during operation. The design topology also avoids the use of a sample-and-hold circuit, but rather uses the capacitor array to store the data before conversion. The lack of a sample-andhold circuit improves the power dissipation of the ADC when compared to published 10-bit SAR ADC architectures.

The design is the first 10-bit SAR ADC that implements a digitally tunable series attenuation capacitor. The tuning involves 5 digital input bits that control switches that either bypass or include capacitors from the series attenuation capacitor circuit. The tuning allowed for the range of the series attenuation capacitor to be from $572 \mathrm{fF}$ to $694 \mathrm{fF}$, resulting in a $122 \mathrm{fF}$ range, for a capacitor whose ideal value is $647 \mathrm{fF}$. Having a tunable series attenuation capacitor meant that the there was a relaxed requirement for ensuring the capacitor is not influenced by the substrate parasitic capacitances. It also allowed the design to use a smaller unit capacitor since matching 
the series attenuation capacitor to the capacitor array was no longer a stringent design requirement. The use of a smaller unit capacitor meant that the entire capacitor array can be smaller and that charging and discharging of the capacitor array can be faster. The increased speed of charging/discharging the capacitor array allowed the sample rate at $1.2 \mathrm{~V}$ to reach $500 \mathrm{kS} / \mathrm{s}$, resulting in a low energy consumption.

Using a 1.2V supply, suitable for the Xilinx Virtex II FPGA, the digital output bits and digital series attenuation capacitor control bits were read into and written from MATLAB. The series attenuation capacitor was tuned using the MATLAB software. Through MATLAB, an FPGA register, connected to the digital capacitor code input to the ADC, was written to. The FPGA was also programmed with $64 \mathrm{kB}$ of RAM in order to store the output for each capacitor code for analysis with MATLAB. Using the MATLAB software and the FPGA, the 5-bit attenuation capacitor was varied and the output was stored in RAM. This output was analyzed and the best code was kept as the operational attenuation capacitance of the ADC.

The chip was designed using a standard $0.13 \mu m$ CMOS technology, with an approximate chip area of $1.4 \mathrm{~mm}^{2}$.

\subsection{Drawbacks of Presented Design}

Successive approximation ADCs have the capability of low power consumption with a high resolution, but not without some drawbacks. The main drawback of an SAR ADC architecture is a low sample rate, when compared to other architectures. However, since the ADC from this work is designed for biomedical, implantable applications, the limited speed is not a problem. 
The implemented SAR architecture has an input voltage range from $\frac{1}{2} V_{d d}$ to $V_{d d}$; therefore, the analog ground must always be at $\frac{1}{2} V_{d d}$. This analog ground signal would likely require a bandgap voltage reference, resulting in an increased power consumption.

Specific to the presented architecture, it was shown that the digital implementation of the attenuation capacitor tuning is not linear. As the capacitor tuning code is increased bit-by-bit from '00000' to ' 11111 ', the attenuation capacitor is decreasing overall, but not at every interval. This means that any automated tuning algorithm must analyze the ADC performance will all possible capacitor codes to find the optimal attenuation capacitance.

In this work, at a $V_{d d}$ of $1.2 \mathrm{~V}$, the attenuation capacitor tuning was done using an FPGA, capable of operating at voltage levels down to $1.5 \mathrm{~V}$, and MATLAB. The supply voltage of the FPGA limited the capabilities of the ADC, since the digital tuning of the capacitor was performed at only one voltage level, $1.2 \mathrm{~V}$. The potential on-chip implementation of a tuning algorithm would allow for a wider range of supply voltages.

\subsection{Future Work}

An important aspect of the presented ADC architecture is the tuning of the attenuation capacitor. As noted earlier, the series attenuation capacitance does not vary linearly while varying the digital input capacitor code. Any future design should attempt to create a tuning range that varies the attenuation capacitance equally for 
each single-bit variation of the digital tuning code. This will make optimization algorithms easier to implement, since the change in capacitance will be predictable for each bit variation in the digital code. A simple attempt to create linear tuning of the attenuation capacitor lead to a 3-bit tuning topology shown in Figure 8.1. If a $650 \mathrm{fF}$ capacitor is desired to be the middle of the code, then $C_{L O W}$ can be set to $620 \mathrm{fF}$ while $\mathrm{C}$ is $10 \mathrm{fF}$, resulting in a range from $620 \mathrm{fF}$ to $690 \mathrm{fF}$. Although $10 \mathrm{fF}$ is a small capacitor that should be designed with a combination of capacitors in series.

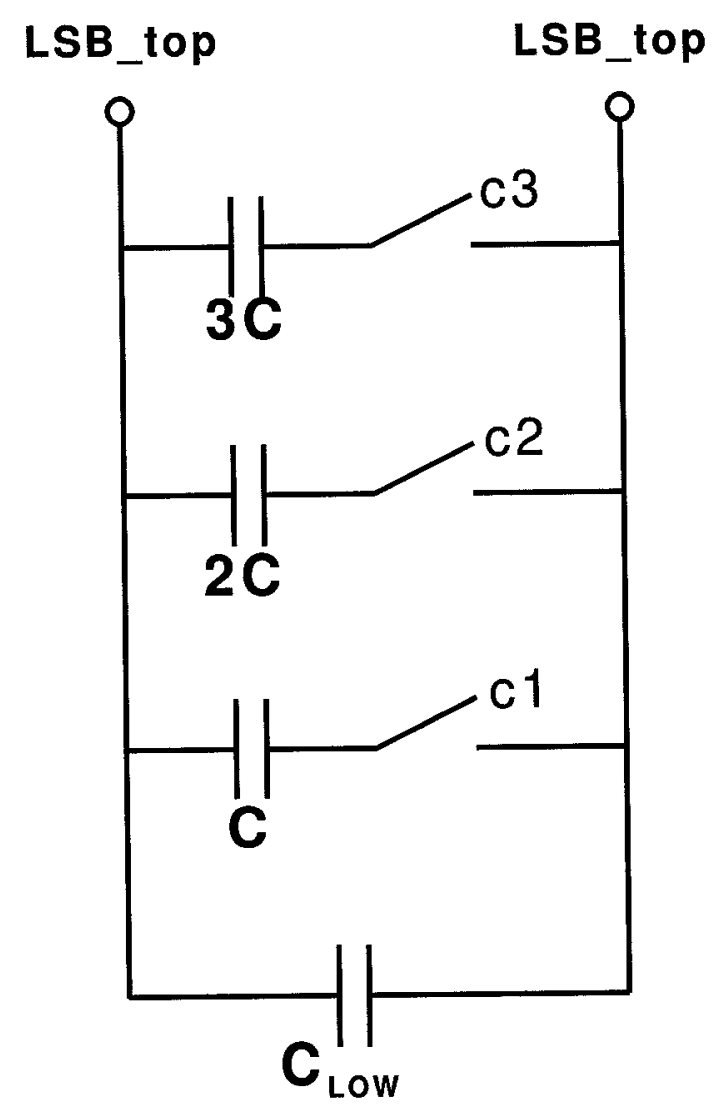

Figure 8.1: Simple 3-bit capacitor tuning topology.

A future implementation of the presented ADC can use a digital tuning algorithm, designed with subthreshold digital logic gates. This digital logic should also be designed so that it draws power only while the algorithm is running; a capability to 
turn the power off after the optimal attenuation capacitor code is found should also be implemented. The chip can be equipped with an on-chip oscillator, a resistive ladder network, or any predictable input, which would be powered only while the algorithm is running to minimize the power drained by the added circuitry. Figure 8.2 is a suggestion of a potential circuit to implement this idea.

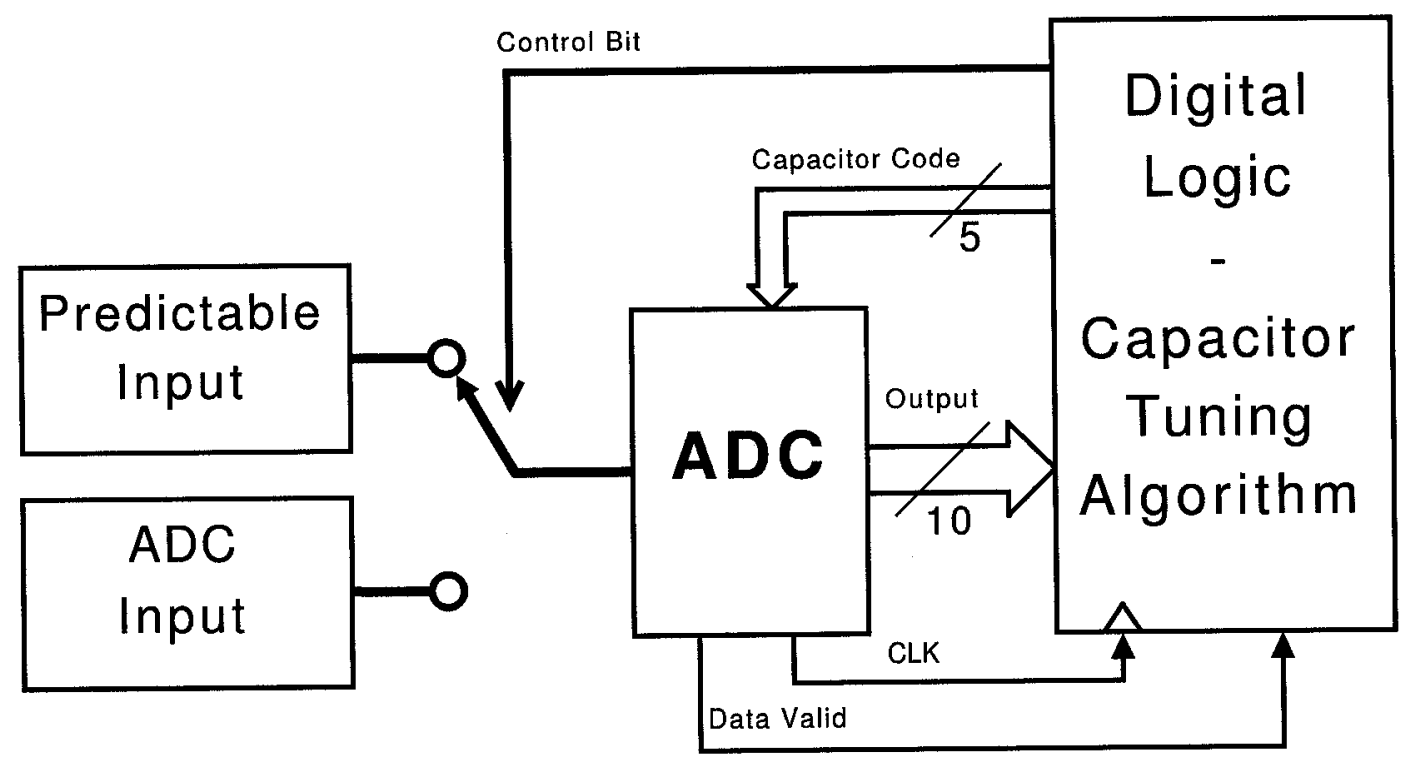

Figure 8.2: Block diagram of a circuit that implements on-chip capacitor tuning.

Although the power dissipation for the presented ADC is low, there can be improvements. Currently, the comparator draws a current, $I_{\text {bias }}$, even when it is not being used. During the sample phase and the valid data output phase of the A/D conversion, the comparator and analog switches are not required, but they are still drawing static power. Having an algorithm to turn off the comparator when it is not being used can improve the overall power dissipation. The comparator is not operational for three clock cycles out of the total 14 cycles required to perform one 10-bit conversion. While operating at $0.6 \mathrm{~V} V_{d d}$, at a $25 \mathrm{kS} / \mathrm{s}$ sample rate, the analog power comprises $94 \%$ of the total power dissipation of the ADC. If this analog block 
can be disconnected for $\frac{3}{14}$ of the time, it would amount to a $20 \%$ saving from the total ADC power dissipation. Assuming that $5 \%$ of the power is irretrievable, a $15 \%$ power saving would result in the ADC performing at $926.5 \mathrm{nW}$ at $0.6 \mathrm{~V}$, sampled at $25 \mathrm{kS} / \mathrm{s}$, resulting in an energy of $36.9 \mathrm{pJ} /$ conversion, $0.15 \mathrm{pJ} /$ state, and $4.7 \mathrm{pJ} / \mathrm{bit}$. 


\section{Appendix A}

\section{Sub-Threshold Design}

\section{A.1 Sub-Threshold MOSFET Operation}

Transistor operation is characterized by its drain current, $I_{D S}$, response to changes in gate-source voltage, $V_{G S}$, and drain-source voltage, $V_{D S}$. Figure A.1(a) illustrates a common $I_{D}-V_{G S}$ curve for the $0.13 \mu m$ CMOS process that is used for the presented ADC design. The NMOS transistor used has a width of $1 \mu \mathrm{m}$ and a length of 250 $\mathrm{nm}$. From the plot, the threshold voltage, $V_{t h}$ is approximately $0.35 \mathrm{~V}$, as is expected for this technology. Figure A.1(a) shows no current flow through the transistor when a $V_{G S}$ that is less than $V_{t h}$ is applied. In Figure A.1(b), it is shown that there is some current present and that it sharply rises at an exponential rate, similar to the bipolar junction transistor (BJT) [39]. When the applied gate voltage is lower than the threshold voltage of the transistor technology used, it is said that the transistor is in the subthreshold mode of operation [39]. This is visible in the cross-section of the MOSFET shown in Figure A.2, where we can see a depletion region, but no channel is formed since $V_{G S}<V_{t h}$. While the transistor is in the subthreshold, or weak inversion, mode of operation, the amount of current flow is minimal when compared to current flow in strong inversion, but there is still conduction present, and this small current is the basis of low-power design.

The primary sources of noise in a MOSFET at low frequencies are thermal noise and flicker noise. Thermal noise is associated with the noise of any resistive substance 

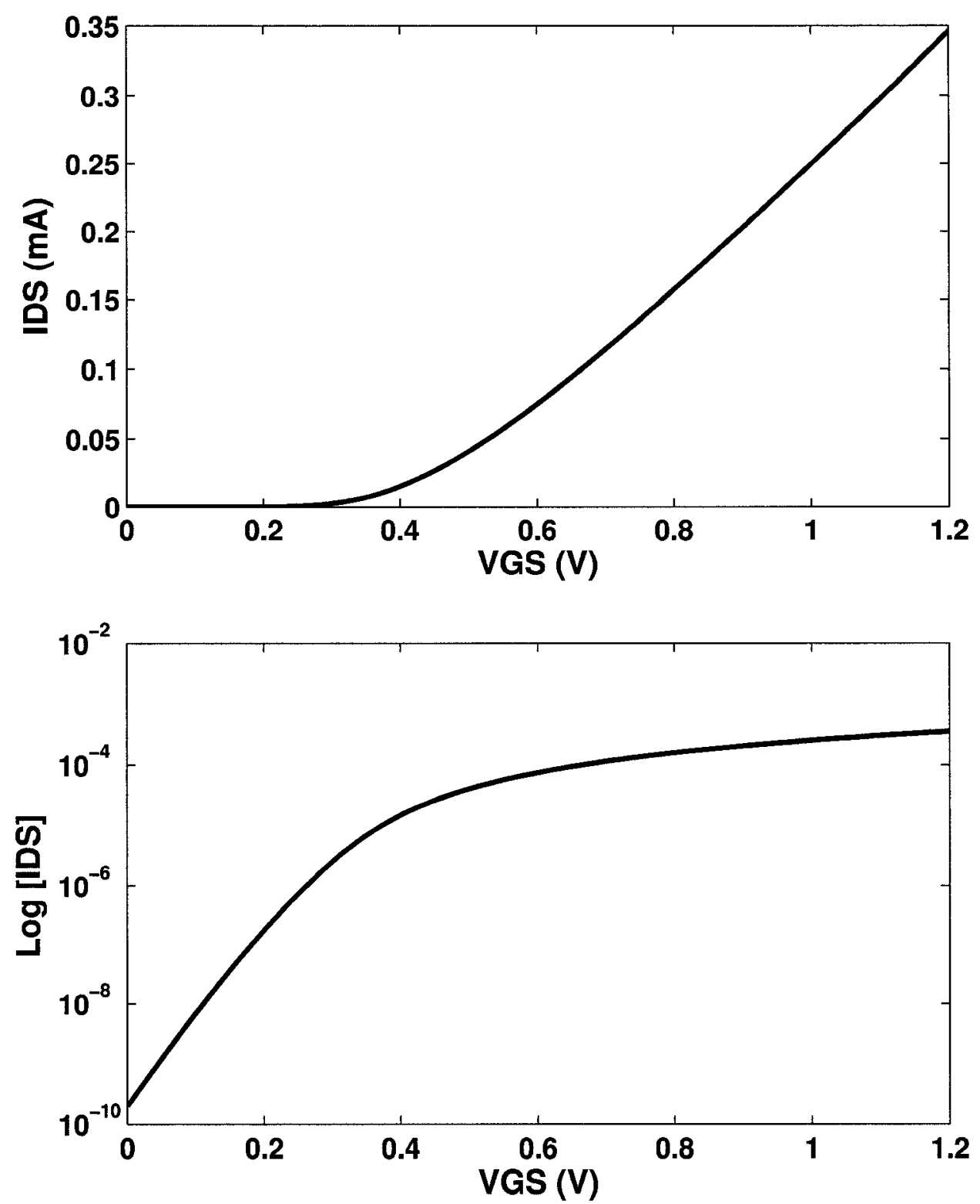

Figure A.1: $I_{D S}$ vs. $V_{G S}$ simulation for an NFET (W=1 $\left.\mu m, \mathrm{~L}=250 \mathrm{~nm}, V_{D S}=1.2 \mathrm{~V}\right)$ 


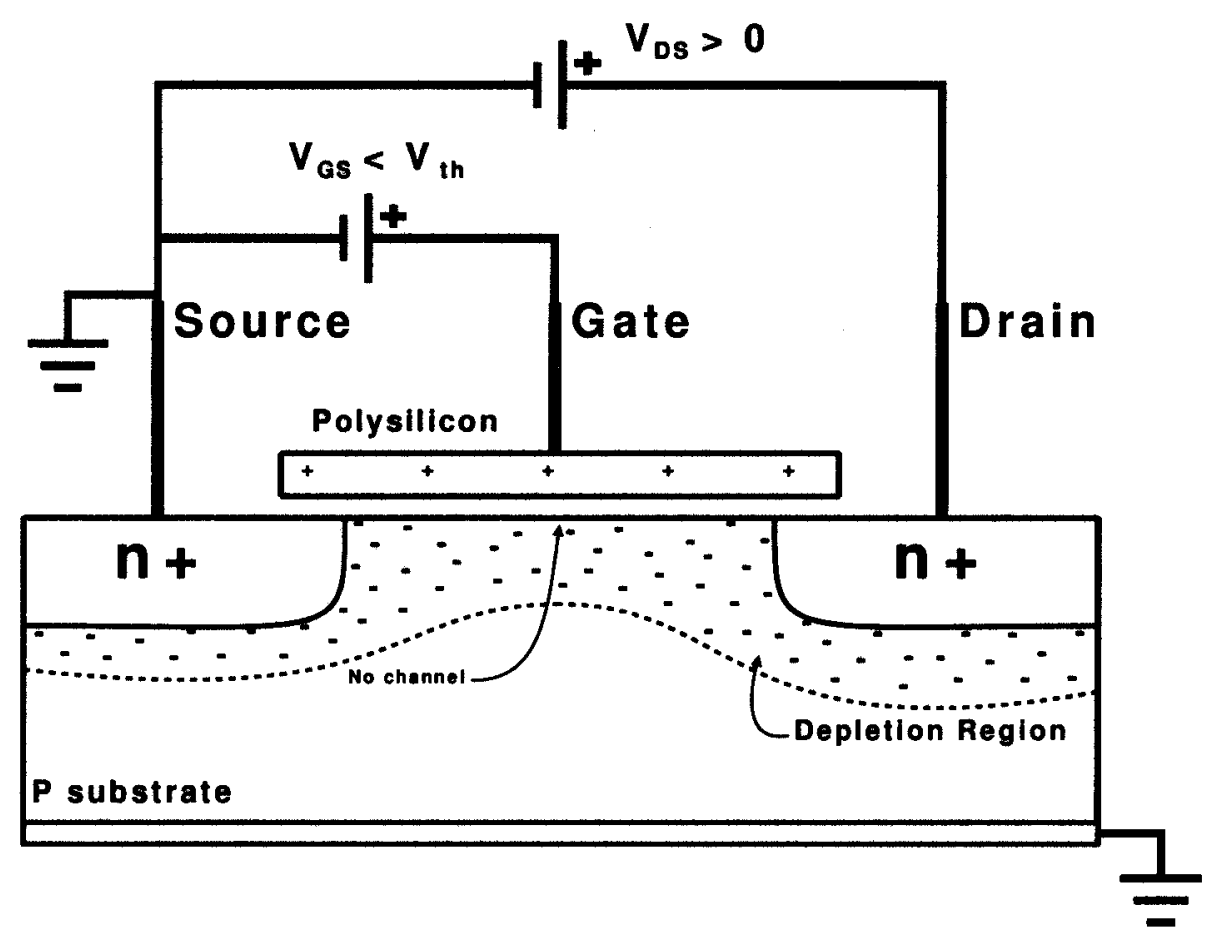

Figure A.2: Cross section of an n-type MOSFET in the subthreshold regime.

and is the result of the random motion of electrons due to thermal effects [24]. The analysis of thermal noise of a transistor in the subthreshold region of operation is derived by viewing the transistor as a series of resistors [40]. The integrated noise of all these resistors yields a net thermal noise after some detailed mathematical manipulation [40]. This noise was analyzed by Mead [40], resulting in the conclusion that the noise is essentially the sum of shot-noise components from the forward and reverse currents, or the total thermal noise. The expression for the total thermal noise was found to be

$$
\Delta I^{2}=2 q I_{s a t}\left(1+e^{\frac{-V_{d s}}{v_{T}}}\right) \Delta f,
$$

where $I_{s a t}$ is the saturation current at the given gate voltage, and $\Delta f$ is the bandwidth of the system.

The main low-frequency noise component in a MOS transistor is flicker noise, also known as $\frac{1}{f}$ noise, which is a low-frequency noise that results from the trapping 
and de-trapping of charges at the oxide to semiconductor interface [48]. The power spectral density of a MOS transistor's $\frac{1}{f}$ noise in the linear region of operation is given in [49] as

$$
S_{V_{G}}(f)=\left(\frac{q}{C_{O X}}\right)^{2} \frac{1}{W L f^{n}} \frac{N_{T}\left(E_{F}\right)}{\gamma},
$$

where $C_{O X}$ is the oxide capacitance, $W$ is the width of the transistor, $L$ is the length of the transistor, $N_{T}\left(E_{F}\right)$ is the equivalent energy of oxide traps at the Fermi Energy level, $q$ is the charge of a single electron, and $\gamma$ is McWhorter's tunneling parameter, which depends on the effective mass of the tunneling carrier and the barrier height [48]. In subthreshold operation, the relationship between $C_{O X}$ and $C_{d}$, the diffusion region capacitance, shown in Figure A.6, needs to be considered since they act in series, resulting in a modified version of Equation A.2:

$$
S_{V_{G}}(f)=\left(\frac{q C_{i n v}}{C_{O X}}\right)^{2} \frac{1}{n^{2}} \frac{q^{2} N_{o t}}{C_{O X} W L} \frac{1}{f}
$$

where $C_{i n v}$ is the inversion capacitance, $N_{o t}$ is the equivalent density of oxide traps, and $n$ is $\left(C_{O X}+C_{d}\right) / C_{O X}$. This capacitor relationship shows that the subthreshold flicker noise will be significantly reduced when compared to saturation flicker noise [48]. It is also suggested that $S_{I_{d}}$, the drain current spectral density is directly proportional to $I^{2}$, while being inversely proportional to the oxide capacitance and transistor gate area [48].

The threshold voltage and mobility of a MOS transistor decrease with increasing temperatures and a decrease in threshold voltage leads to an increase in drain current at near-threshold $V_{G S}$ and subsequent increase in power dissipation [24]. A CMOS inverter with a NMOS transistor with $\mathrm{W} / \mathrm{L}$ of $1 \mu \mathrm{m} / 250 \mathrm{~nm}$ and a PMOS transistor with $\mathrm{W} / \mathrm{L}$ of $3.5 \mu \mathrm{m} / 250 \mathrm{~nm}$ was simulated for temperature effects. Varying the temperature from $0^{\circ} \mathrm{C}$ to $125^{\circ} \mathrm{C}$ for the inverter, with a $V_{D S}$ of $0.4 \mathrm{~V}$ and $V_{G S}$ of $0.2 \mathrm{~V}$, causes the drain current to increase from $37 \mathrm{nA}$ to $842 \mathrm{nA}$. This is a significant increase when compared to a simulation with a $V_{D S}$ of $1.2 \mathrm{~V}$ and $V_{G S}$ of $0.6 \mathrm{~V}$, which resulted 
in an increase in drain current from $61 \mu \mathrm{A}$ to $69 \mu \mathrm{A}$, or a $13 \%$ increase. Increasing temperature also affects the inverter OFF current, $I_{\text {off }}$ and the subthreshold voltage transfer characteristic (VTC) slope. The increasing current causes the VTC slope to also increase, resulting in a faster circuit that dissipates more power [3].

\section{A.1.1 Analog Design in Subthreshold}

In order to be able to design analog circuits in the subthreshold regime, knowing the current response to changes in $V_{G S}$, or the slope of Figure A.1(b), is essential. This current-voltage relationship is given in [50] as

$$
I_{d s}=\mu_{e f f} C_{o x} \frac{W}{L}\left(\frac{k T}{q}\right)^{2} e^{\frac{\left(v_{g s}-V_{t}\right)}{m k T / q}}\left(1-e^{-\frac{V_{d s}}{k T / q}}\right)
$$

where,

$\mu_{\text {eff }}$ - effective electron/hole mobility;

$C_{o x}$ - oxide capacitance;

$C_{d}$ - depletion region capacitance;

$W$ - transistor width;

$L$ - transistor length;

$m-1+\frac{C_{d}}{C_{o x}}$;

$\frac{k T}{q}$ - thermal voltage;

$V_{g s}$ - gate-source voltage;

$V_{t}$ - threshold voltage;

$V_{d s}$ - drain-source voltage

The exponential term $\left(1-e^{-\frac{V_{d s}}{k T / q}}\right)$ is only a factor in the equation for $I_{d s}$ if $V_{d s}$ is less than $3 v_{t}$ or $3 \frac{k T}{q}$. When $V_{d s}$ is greater than $3 v_{t}$, or grater than approximately 
$75 \mathrm{mV}$ at room temperature, the exponential term $\left(1-e^{-\frac{V_{d s}}{k T / q}}\right)$ ceases to be a significant influence on the drain current [18], reducing Equation A.4 to

$$
I_{d s}=\mu_{e f f} C_{o x} \frac{W}{L}\left(\frac{k T}{q}\right)^{2} e^{\left[\frac{\left(v_{g s}-v_{t}\right)}{m k T / q}\right]} .
$$

If we collect the constant terms into $I_{0}$ and replace the thermal voltage $\frac{k T}{q}$ with its symbol, $v_{t}$, Equation A.5 becomes

$$
I_{d s}=I_{0} \frac{W}{L} e^{\left[\frac{\left(v_{g s}-v_{t}\right)}{m v_{t}}\right]}
$$

The relationship between $I_{d s}$ and $v_{g s}$ from Equation A.6 is very similar to the equation for the collector current, $I_{C}$ in a bipolar junction transistor (BJT) and its relationship with the base voltage, $V_{B E}$. The similarity is evident, as the equation for the collector current in a BJT is given by [51] as

$$
I_{C}=I_{S} e^{\left[\frac{v_{B E}}{v_{t}}\right]}
$$

The simplified subthreshold $I_{d s}$ current equation for a MOSFET, shown in Equation A.6 suggests that the transistor can function like a BJT. A plot of the drain current as it relates to $V_{d s}$ is shown in Figure A.3, where the potential for designing analog circuits in subthreshold becomes evident. In Figure A.3 it is shown that the device can function like a BJT or a MOSFET operating in active or saturation regions respectively.

The difference between designing with a BJT and a subthreshold MOSFET is that, due to the low current, the MOSFET device will operate with a very low transconductance, $g_{m}$. The equation for the transconductance in a subthreshold MOSFET is derived in [24] as

$$
g_{m}=\frac{I_{D}}{n V_{T}}
$$




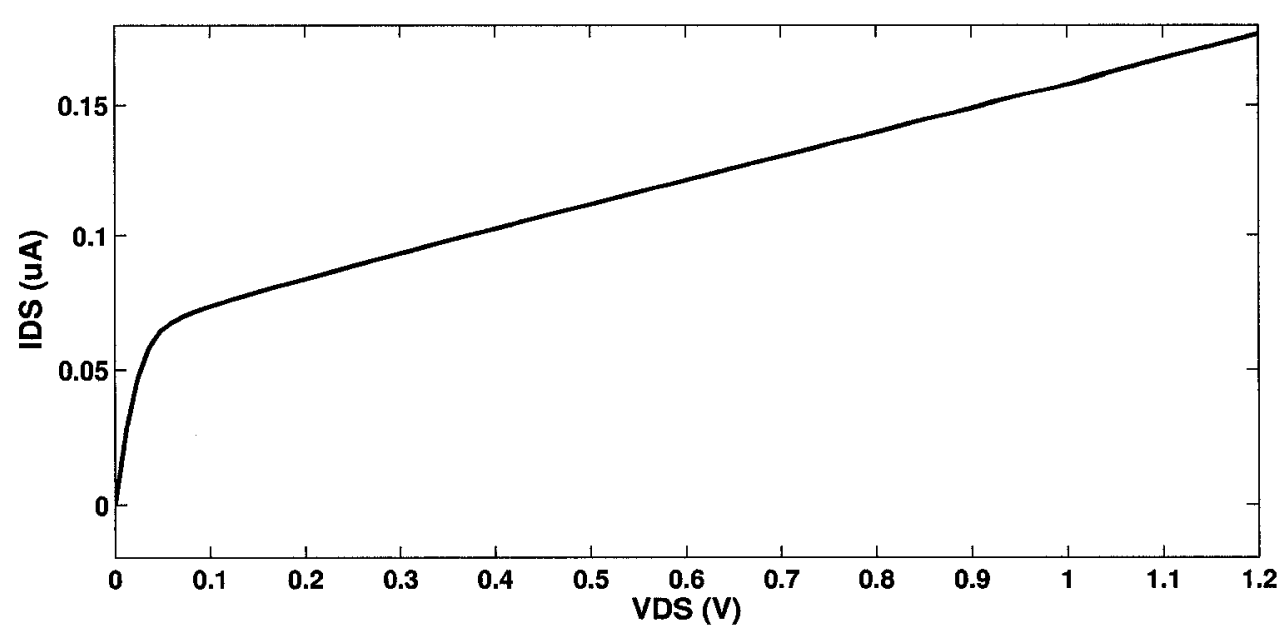

Figure A.3: $I_{D S}$ vs. $V_{D S}$ simulation for an NFET. $\left(\mathrm{W}=1 \mu m, \mathrm{~L}=250 \mathrm{~nm}, V_{G S}=0.2 \mathrm{~V}\right)$

Equation A.8 suggests that $g_{m}$ increases linearly with an increasing current in the subthreshold region of operation, while it is proportional to the squared root of the drain current as $g_{m}=\sqrt{\beta_{n} I_{D}}$ in saturation [24]. The low $g_{m}$ value results in a transition frequency that is much lower than the frequency for a BJT in the active region as well as a MOSFET in the saturation region, since $g_{m}$ is directly proportional to the transition frequency, $f_{t}$ [24]. The $f_{t}$ for a subthreshold MOSFET in $0.13 \mu m$ CMOS is in the $\mathrm{MHz}$ and low $\mathrm{GHz}$ range, while in saturation, it is approximately $100 \mathrm{GHz}[18]$.

\section{A.1.2 Digital Design in Subthreshold}

Without major alterations of a standard CMOS digital circuit and by reducing the supply voltage below the threshold voltage, $V_{t}$, a digital circuit can function using only subthreshold leakage currents. This circuit operates at a fraction of the power when compared to a circuit with devices in the saturation region of operation [50]. In addition to the lowered power dissipation, subthreshold transistors have an advantage in that they act as constant current sources, independent of the drain voltage, as long as the drain voltage is larger than $3 \frac{k T}{q}$. Transistors in saturation have high gain as 
the drain current varies along with the drain voltage [39]. This behaviour is shown in [50] and reproduced in Figure A.4 for a $0.13 \mu \mathrm{m}$ CMOS inverter. In Figure A.4 it is shown that lower $V_{d s}$ voltages result in sharper corners for the voltage transfer characteristic (VTC) curve. As $V_{d s}$ is increased, the corners of the VTC curve start to round off, suggesting a sharper VTC for an inverter operating in the subthreshold region of operation [50].

The power savings when using subthreshold digital circuits are shown in Figure A.5, where it becomes evident that the drain current of a CMOS inverter, while sweeping $V_{g s}$, is significantly smaller for the subthreshold inverter. This is expected, as the average dynamic power dissipated by a CMOS inverter is given in [24] as

$$
P_{a v g}=C_{t o t} V_{D D}^{2} f_{c l k}
$$

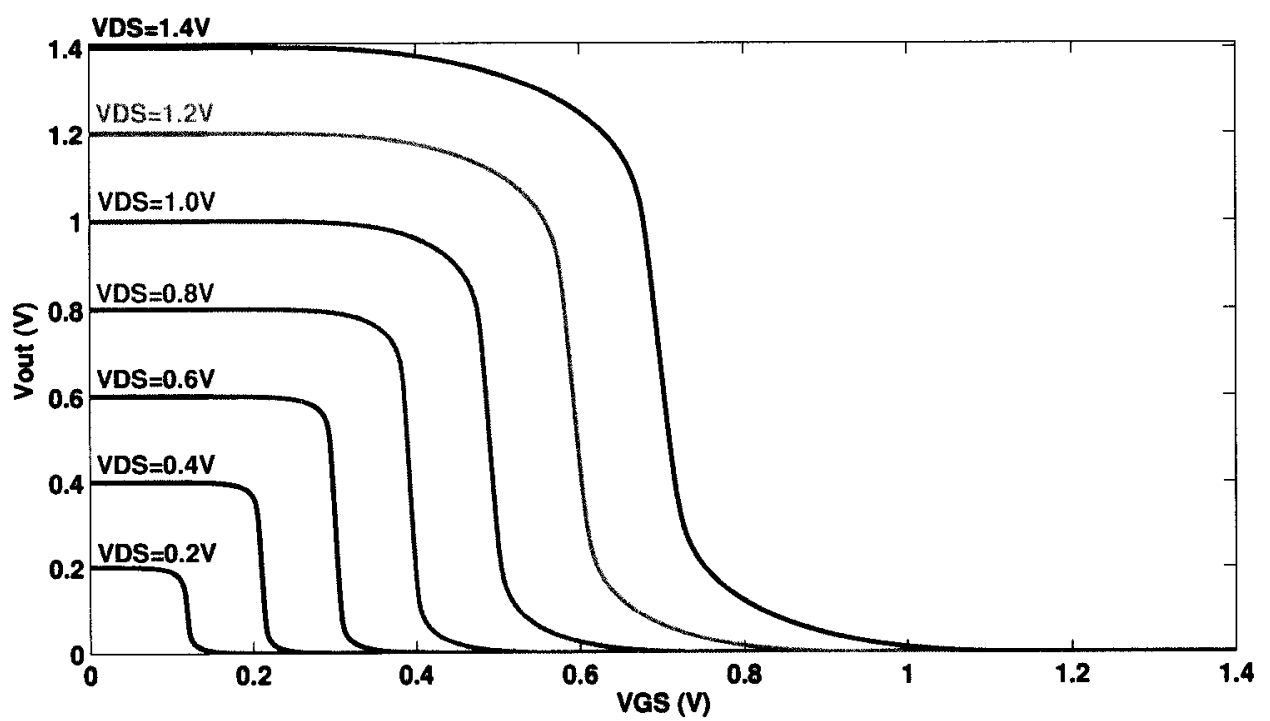

Figure A.4: Voltage Transfer Characteristics (VTC) for an inverter with varying $V_{D S}$ voltages. The inverter is a CMOS inverter with an NMOS transistor with $\mathrm{W}=1 \mu \mathrm{m}$, $\mathrm{L}=250 \mathrm{~nm}$, and PMOS transistor with $\mathrm{W}=3.5 \mu \mathrm{m}, \mathrm{L}=250 \mathrm{~nm}$.

In the subthreshold region of operation, the transistor input capacitance is less than the input capacitance of a transistor in strong inversion. While the input capacitance of a MOSFET in strong inversion operation is dominated by the oxide 


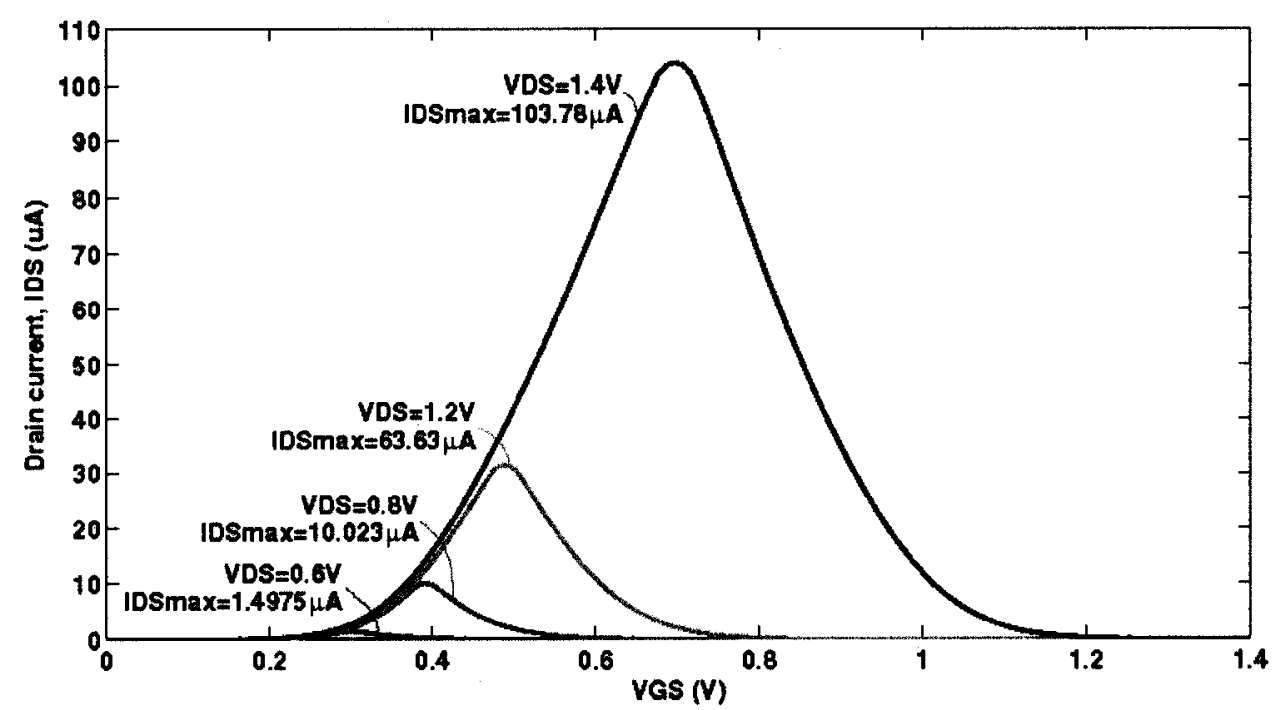

Figure A.5: Drain current for an inverter while sweeping $V_{g s}$ with various $V_{D S}$ voltages. The inverter is a CMOS inverter with an NMOS transistor with $\mathrm{W}=1 \mu \mathrm{m}$, $\mathrm{L}=250 \mathrm{~nm}$, and PMOS transistor with $\mathrm{W}=3.5 \mu \mathrm{m}, \mathrm{L}=250 \mathrm{~nm}$.

capacitance, the subthreshold region MOSFET capacitors are a combination of the capacitors shown in Figure A.6, resulting in a total input capacitance, $C_{i}$, given in [3] by

$$
C_{i}=\frac{C_{o x} C_{d}}{C_{o x}+C_{d}}+C_{i f}+C_{o f}+C_{d o}
$$

The main reason behind the low input capacitance is the depletion region capacitance. The largest individual capacitance is the oxide capacitance, $C_{o x}$. The depletion region capacitance, $C_{d}$, is very low, and having this in series with $C_{O X}$ effectively eliminates the effects of $C_{O X}$.

The combination of lowered $V_{D D}$ and lowered input capacitance results in the ability of the subthreshold digital circuit to operate at low power dissipation. The VTC of an inverter in the subthreshold region of operation is closer to the ideal when compared to an inverter in the strong inversion region. This improvement is caused by an increase in the circuit gain. [52]. The transconductance, $g_{m}$, is increased as a result of the exponential relationship between $I_{d s}$ and $V_{g s}$ [52], as shown in Equation A.5. 


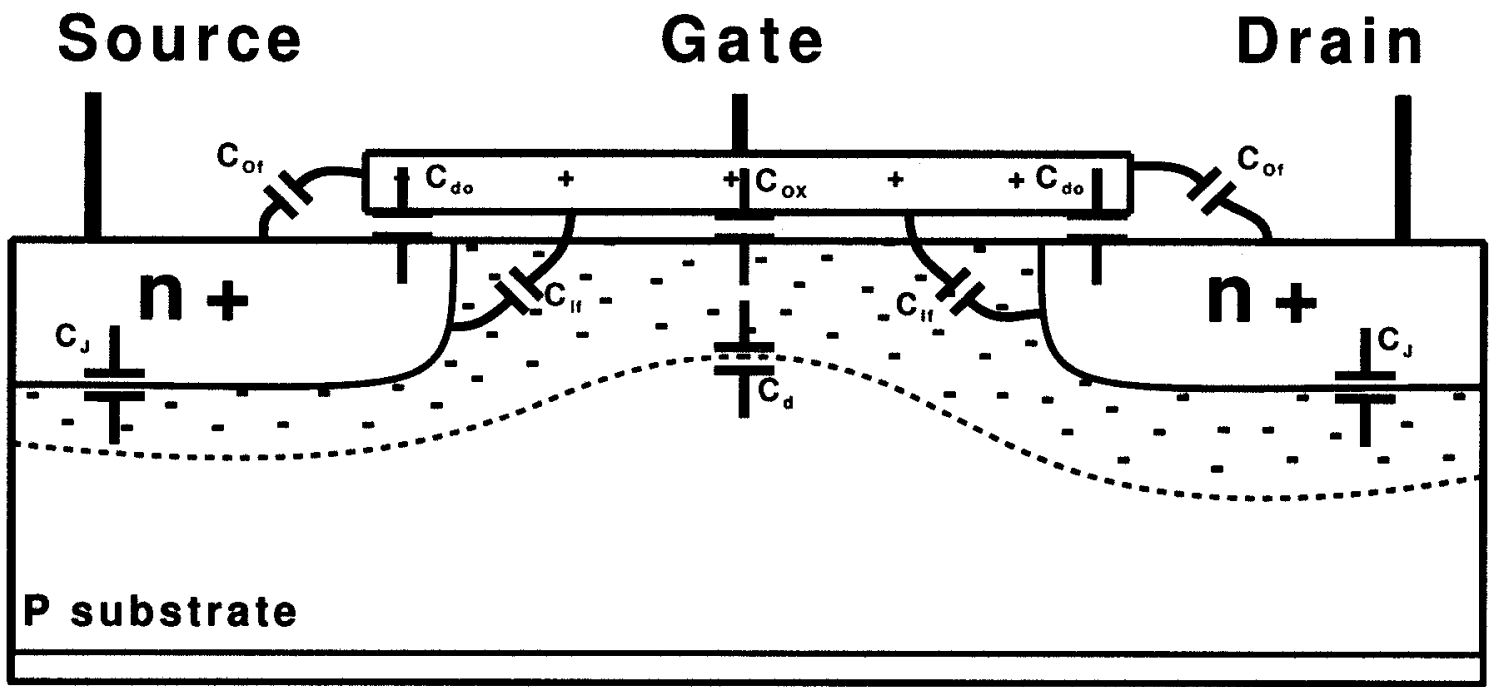

Figure A.6: Capacitor components for an n-type MOSFET in the subthreshold regime [3]. $C_{O X}$ is the oxide capacitance, $C_{O f}$ and $C_{i f}$ are fringing capacitances, $C_{d o}$ are the drain and source overlap capacitances, $C_{d}$ is the depletion region capacitance, and $C_{J}$ is the junction capacitance. 


\section{Appendix B}

\section{Resulting Publications}

As a result of the work presented in this thesis, the following paper was published at the International Conference on Microelectronics:

L. Filipovic, L. MacEachern, "A 10-bit Low-Power SAR ADC With a Tunable Series Attenuation Capacitor", International Conference on Microelectronics (ICM), Dec 2008. (in press) 


\section{Bibliography}

[1] R. Viswanathan and P. Varshney, "Distributed detection with multiple sensors: I. fundamentals," Proceedings of the IEEE, vol. 85, no. 1, pp. 54-63, Jan 1997.

[2] N. Gray, "ABCs of ADCs - Analog-to-Digital Converter Basics," National Semiconductor Corporation, USA, Tech. Rep., 2006, available at http://www.national.com/appinfo/adc/files/ABCs_of_ADCs.pdf.

[3] A. Raychowdhury, B. Paul, S. Bhunia, and K. Roy, "Computing with Sub-threshold Leakage: Device/Circuit/Architecture Co-Design for Ultralow-Power Sub-threshold Operation," IEEE transactions on Very Large Scale Integration (VLSI Systems), vol. 13, no. 11, pp. 1213-1224, November 2005.

[4] V. S. Kappagantu, "Wireless Sensors for In-Home Medical Management," Master's thesis, State University of New York at Buffalo, February 2008.

[5] B. K. Malachira, "Low-Power Sampled-Data Dual-Slope ADC," Master's thesis, The University of Texas at Dallas, 2007.

[6] P. Varshney, Distributed Detection and Data Fusion. New York: Springer-Verlag.

[7] S. Gambini and J. Rabaey, "A $100 \mathrm{ks} / \mathrm{s} 65 \mathrm{db}$ dr sigma-delta adc with $0.65 \mathrm{v}$ supply voltage," in Solid State Circuits Conference, 2007. ESSCIRC 2007. 33rd European, Sept. 2007, pp. 202-205.

[8] J. Goes, N. Paulino, H. Pinto, R. Monteiro, B. Vaz, and A. Garcao, "Low-Power LowVoltage CMOS A/D Sigma-Delta Modulator for Bio-Potential Signals Driven by a Single-Phase Scheme," in IEEE Transactions on Circuits and Systems, vol. 52, no. 12, December 2005, pp. 1058-1067.

[9] R. Amirtharajah and A. Chandrakasan, "Self Powered Signal Processing Using Vibration Based Power Generation," in IEEE Journal of Solid-State Circuits, vol. 33, no. 5, May 1998, pp. 687695.

[10] A. Fish, S. Hamami, and O. Yadid-Pecht, "CMOS Image Sensors With Self-Powered Generation Capability," in IEEE Transactions on Circuits and Systems II: Express Briefs, vol. 53, no. 11, November 2006, pp. 1210-1214.

[11] T. Starner, "Human Powered Wearable Computing," in IBM Systems Journal, vol. 35, no. 3, 1996, pp. 618-629.

[12] Z. Fu and E. Culurciello, "An ultra-low power silicon-on-sapphire adc for energy-scavenging sensors," in Circuits and Systems, 2006. ISCAS 2006. Proceedings. 2006 IEEE International Symposium on, 2006, pp. 1511-1514.

[13] M. D. Scott, B. Boser, and K. Pister, "An Ultralow-Energy ADC for Smart Dust," IEEE Journal of Solid-State Circuits, vol. 38, no. 7, pp. 1123-1129, July 2003.

[14] J. Sauerbrey, D. Schmitt-Landsiedel, and R. Thewes, "A 0.5V, $1 \mu \mathrm{w}$ Successive Approximation ADC," IEEE Journal of Solid-State Circuits, vol. 38, no. 7, pp. 1261-1265, July 2003. 
[15] L. S. Y. Wong, S. Hossain, A. Ta, J. Edvinsson, D. Rivas, and H. Naas, "A Very Low-Power CMOS Mixed-Signal IC for Implantable Pacemaker Applications," IEEE Journal of Solid-State Circuits, vol. 39, no. 12, pp. 2446-2456, December 2004.

[16] H. Yang and R. Sarpeshkar, "A Bio-Inspired Ultra-Energy-Efficient Analog-to-Digital Converter for Biomedical Applications," in IEEE Transactions on Circuits and Systems, vol. 53, no. 11, November 2005, pp. 2349-2356.

[17] Z. Fu and E. Culurciello, "An Ultra-Low Power Silicon-On-Sapphire ADC for EnergyScavenging Sensors," in ISCAS '06. Proceedings of the 2006 International Symposium on Circuits and Systems, vol. 1, 2006, pp. 1511-1514.

[18] K. Abdelhalim, "Ultra Low Power Analog to Digital Converter for Biomedical Applications," Master's thesis, Carleton University, 2007.

[19] J. Nielsen and E. Bruun, "A Low-Power 10-bit Continuous-Time CMOS Sigma Delta AD Converter," in IEEE Transactions on Circuits and Systems, vol. 1, May 2004, pp. 174-178.

[20] G. Bonfini, A. S. Brogna, C. Garbossa, L. Colombini, M. Bacci, S. Chicca, F. Bigongiari, N. C. Guerrini, and G. Ferri, "An Ultralow-Power Switched Opamp-Based 10-b Integrated ADC for Implantable Biomedical Applications," in IEEE Transactions on Circuits and Systems, vol. 51, no. 1, January 2004.

[21] H.-C. Chow and Y.-H. Chen, "1v 10-bit successive approximation adc for low power biomedical applications," in 18th European Conference on Circuit Theory and Design, 2007. ECCTD 2007, 27-30 Aug. 2007, pp. 196-199.

[22] E. Lopez-Morillo, R. Carvajal, F. Munoz, H. El Gmili, A. Lopez-Martin, J. Ramirez-Angulo, and E. Rodriguez-Villegas, "A 1.2-v 140-nw 10-bit sigmadelta modulator for electroencephalogram applications," Biomedical Circuits and Systems, IEEE Transactions on, vol. 2, no. 3, pp. 223-230, Sept. 2008.

[23] AN810, "Understanding Flash ADCs," Maxim Electronics, USA, Tech. Rep., October 2001, available at http://pdfserv.maxim-ic.com/en/an/AN810.pdf.

[24] R. Jacob Baker, CMOS: Circuit Design, Layout, and Simulation, 2nd ed. John Wiley and Sons, 2005.

[25] J. Terada, Y. Matsuya, F. Morisawa, and Y. Kado, "8-mw, 1-v, 100-msps, 6-bit A/D Converter Using a Transconductance Latched Comparator," in IEEE Asia Pacific Conference on ASIC, 2000 , pp. 53-56.

[26] C.-C. Tsai, K.-W. Hong, Y.-S. Hwang, W.-T. Lee, and T.-Y. Lee, "New Power Saving Design Method for CMOS Flash ADC," in IEEE International Midwest Symposium on Circuits and Systems, 2004, pp. III-371 - III-374.

[27] Y.-S. Hwang, J.-F. Lin, C.-C. Huang, J.-J. Chen, and W.-T. Lee, "An Efficient Power Reduction Technique for Flash ADC," in SOC Conference, 2007 IEEE International, September 2007, pp. $43-46$.

[28] R. Wang, "A Low Voltage Low Power 10-bit Pipeline ADC in 90nm CMOS Technology," Master's thesis, University of Toronto, 2004.

[29] Y. M. Lourans Samid, "A Low Power and Low Voltage Continuous Time $\sum \triangle$ Modulator," in ISCAS 2008. IEEE International Symposium on Circuits and Systems, May 2008, pp. 18821885.

[30] X. Yuan, N. Tran, and S. Signell, "On Low Power Design of Feedforward Continuous-Time Sigma Delta Modulators With Excess Loop Delay," in ISCAS 2008. IEEE International Symposium on Circuits and Systems, May 2008, pp. 1882-1885.

[31] D. A. Johns and K. Martin, Analog Integrated Circuit Design. Wiley, 1997. 
[32] S. Gambini and J. M. Rabaey, "Low Voltage Analog to Digital Converter Design in 90nm CMOS," Master's thesis, EECS Department, University of California, Berkeley, Jan 2007. [Online]. Available: http://www.eecs.berkeley.edu/Pubs/TechRpts/2007/EECS-2007-17.html

[33] A. Agarwal, "Low Power Current Mode ADC for CMOS Sensor IC," Master's thesis, Texas AM University, 2005.

[34] H. Le, J. Singh, L. Hiremath, V. Mallapur, and A. Stojcevski, "Ultra-Low-Power VariableResolution Successive Approximation ADC for Biomedical Application," in Electronic Letters, vol. 41, no. 11, May 2005.

[35] K. Abdelhalim, L. MacEachern, and S. Mahmoud, "A Nanowatt ADC for Ultra-Low-Power Applications," in ISCAS '06. Proceedings of the 2006 International Symposium on Circuits and Systems, vol. 1, 2006, pp. 617-620.

[36] R. Gregorian, CMOS Op-Amps and Comparators. New York, NY: John Wiley and Sons, 1999.

[37] ES\#57P9006, "CMOS8RF (CMRF8SF) Design Manual," Department BEVV, Mixed Signal Technology Development, IBM Microelectronics Division, Tech. Rep., April 24, 2007.

[38] T. Anderson, "Optimum Control Logic For Successive Approximation A-C Converters," Computational Design, vol. 11, no. 7, pp. 81-86, July 1972.

[39] Jan M. Rabaey and Anantha Chandrakasan and Borivoje Nikolic, Digital Integrated Circuits, 2nd ed. Prentice Hall Electronics and VLSI Series, 2003.

[40] R. Sarpeshkar, T. Delbruck, and C. A. Mead, "White Noise in MOS Transistors and Resistors," IEEE Circuits and Devices Magazine, vol. 9, pp. 23-29, November 1993.

[41] K. Abdel-Halim, L. MacEachern, and S. Mahmoud, "A Nanowatt Successive Aproximation ADC With Offset Correction for Implantable Sensor Applications," in Proceedings of the 2007 International Symposium on Circuits and Systems, 2007. ISCAS 07, vol. 1, May 2007, p. 23512354.

[42] E. Culurciello and A. Andreou, "An 8-bit, $1 \mathrm{~mW}$ Successive Approximation ADC in SOI CMOS," in ISCAS '03. Proceedings of the 2003 International Symposium on Circuits and Systems, vol. 1, 2003, pp. 301-304.

[43] M. D. Scott, "An Ultra Low-Power ADC for Distributed Sensor Networks," Master's thesis, University of California at Berkeley, 2003.

[44] AN283, "INL/DNL Measurements for High Speed ADCs," Maxim Electronics, USA, Tech. Rep., 2005, available at http://pdfserv.maxim-ic.com/en/an/AN283.pdf.

[45] "Measuring of dynamic figures: SNR, THD, SFDR," Strategic Test, Tech. Rep., November 2007, available at http://www.strategic-test.com/support/download.

[46] H.-H. Ou, Y.-C. Chen, and B.-D. Liu, "A 0.7-v 10-bit 3uw analog-to-digital converter for implantable biomedical applications," in Biomedical Circuits and Systems Conference, 2006. BioCAS 2006. IEEE, 29 2006-Dec. 1 2006, pp. 122-125.

[47] H. Kim, Y. Min, Y. Kim, and S. Kim, "A low power consumption 10-bit rail-to-rail sar adc using a c-2c capacitor array," in Electron Devices and Solid-State Circuits, 2008. EDSSC 2008. IEEE International Conference on, Dec. 2008, pp. 1-4.

[48] Y. Nemirovsky, I. Brouk, and C. Jakobson, "1/f Noise in CMOS Transistors for Analog Applications," IEEE transactions on Electron Devices, vol. 48, no. 5, pp. 927-927, May 2001.

[49] J. Chang, A. A. Abidi, and C. R. Viswanathan, "Flicker Noise in CMOS Transistors from Subthreshold to Strong Inversion at Various Temperatures," IEEE transactions on Electron Devices, vol. 41, no. 11, pp. 1965-1971, November 1994. 
[50] C. H.-I. Kim, H. Soeleman, and K. Roy, "Ultra Low Power DLMS Adaptive Filter for Hearing Aid Applications," IEEE transactions on Very Large Scale Integration (VLSI Systems), vol. 11, no. 6, pp. 1058-1067, December 2003.

[51] A. S. Sedra and K. C. Smith, Microelectronic Circuits, 5th ed. Oxford University press, 2004.

[52] H. Soeleman and K. Roy, "Ultra-Low Power Digital Subthreshold Logic Circuits," in Low Power Electronics and Design, 1999. Proceedings. 1999 International Symposium on, January 1999, pp. 94-96. 